\title{
25th European Workshop for Rheumatology Research
}

\author{
Glasgow, UK

\section{4-27 February 2005}

Received: 11 January 2005 Published: 17 February 2005

(C) 2005 BioMed Central Ltd

\section{Speaker abstracts}

\section{S1}

Stem cells now and in the future in medicine

A Smith

MRC Centre Development in Stem Cell Biology, Institute for Stem Cell

Research, University of Edinburgh, UK

Arthritis Res Ther 2005, 7(Suppl 1):S1 (DOI 10.1186/ar1506)

Abstract not submitted.

\section{S2}

\section{Chaperonins and the regulation of immunity GS Panayi, vc Corrigall}

Academic Department of Rheumatology, King's College London, Guy's Hospital, London, UK

Arthritis Res Ther 2005, 7(Suppl 1):S2 (DOI 10.1186/ar1507)

Chaperonins have classically been thought of as intracellular molecules involved in the correct folding of proteins. Their expression is upregulated during times of stress such as heat (hence their common nomenclature as heat shock proteins [HSP]), anoxia, hypoglycaemia and reactive oxygen species [1]. These are conditions found in infected tissues or in tissues with chronic inflammation such as the rheumatoid synovium. In their intracellular location they protect the cell from apoptotic death due to stress. Increasingly chaperonins have been recognised to subserve extracellular functions for which they have received the name 'chaperokines' since they bind to specific receptors on the cell surface and activate cells of the innate immune system to secrete inflammatory cytokines, chemokines and small molecular weight mediators such as prostaglandins [2]. Indeed, an early event in inflammation is cell stress/necrosis leading to the release of HSP60 and HSP70 that binds via a CD14-mediated mechanism to Toll-like receptors 2 and 4 [2] as part of the 'danger' signal [3]. The secretion of tumour necrosis factor alpha, IL-1, IL-12 and other chemokines prepares the environment for a TH1 adaptive immune response. It is now recognised that some chaperonins, such as $\mathrm{BiP}$ and HSP27, may activate the innate immune system to secrete anti-inflammatory cytokines, such as IL-10 [4,5] that may skew the adaptive immune response to TH2. Recent work by our group has shown that BiP can not only prevent but also treat ongoing collagen-induced arthritis in DBA/1 mice [6], suggesting that chaperonins may down modulate ongoing $\mathrm{TH} 1$ responses. Thus, it may be possible to suppress rheumatoid inflammation by administration of appropriate chaperonins such as BiP. Finally, chaperonins may be important system regulators determining the outcome between TH1 and Th2 immune responses. References

1. Pockley AG: Heat shock proteins as regulators of the immune response. Lancet 2003, 362:469-476.

2. Asea A: Chaperokine-induced signal transduction pathways. Exerc Immunol Rev 2003, 9:25-33.

3. Matzinger P: The danger model: a renewed sense of self. Science 2002, 296:301-305.

4. De AK, Kodys KM, Yeh BS, Miller-Graziano C: Exaggerated human monocyte IL-10 concomitant to minimal TNF-alpha induction by heatshock protein 27 (Hsp27) suggests Hsp27 is primarily an antiinflammatory stimulus. J Immunol 2000, 165:3951-3958.

5. Corrigall VM, Bodman-Smith MD, Brunst M, Cornell H, Panayi GS: Inhibition of antigen-presenting cell function and stimulation of human peripheral blood mononuclear cells to express an antiinflammatory cytokine profile by the stress protein BiP: relevance to the treatment of inflammatory arthritis. Arthritis Rheum 2004, 50:1164-1171.

6. Panayi G, Brownlie R, Corrigall V, Bodman-Smith M, Thompson S: Immunotherapy of collagen induced arthritis with the stress protein BiP. FASEB J 2004, 18(suppl S):A1174.

Acknowledgements GSP and VCC are shareholders in Immune Regulation Ltd that is investigating the potential of BiP as an immunotherapeutic agent for the treatment of rheumatoid arthritis.
S3

\section{Autoimmune diabetes: mechanisms}

A Cooke

University of Cambridge, UK

Arthritis Res Ther 2005, 7(Suppl 1):S3 (DOI 10.1186/ar1508)

Type 1 diabetes is an autoimmune disease that is increasing dramatically in incidence in the developed world. Explanations for this increase in incidence include viral precipitation of disease and the so-called 'hygiene hypothesis'.

In Type 1 diabetes the insulin-producing beta cells of the pancreas are selectively destroyed by the immune system. Type 1 diabetes is generally recognised as a Tcell-mediated autoimmune disease with the autoantibody response to islet antigens serving as markers of ongoing beta cell destruction.

Studies in spontaneous animal models of this human condition suggest that it is a Th1-mediated autoimmune disease with an involvement of both $C D 8^{+} T$ cells and macrophages in pathology. There are multiple ways in which the beta cell could be destroyed including a Class I restricted killing by $\mathrm{CD}^{+} \mathrm{T}$ cells, proinflammatory cytokine-mediated cell death and death induced by Fas/FasL interactions. At the time of clinical diagnosis it is possible that the patient will have destroyed $70 \%$ of their beta cell mass and exogenous insulin is required to maintain glucose homeostasis. There is currently much interest in the development of tolerogenic strategies to inhibit and halt beta destruction. This coupled with strategies to facilitate the replacement of the destroyed beta cells would constitute a cure for this disease.

S4

Autoimmunity or immune deficiency? On the role and function of regulatory $\mathrm{T}$ cells in chronic arthritis

BJ Prakken, I de Kleer

Department of Paediatric Immunology, University Medical Centre Utrecht, The Netherlands

Arthritis Res Ther 2005, 7(Suppl 1):S4 (DOI 10.1186/ar1509)

Human autoimmune diseases are characterized by chronic, non-remitting inflammation. In the early phase of chronic arthritis auto-reactive T cells initiate a cascade of events that leads to chronic inflammation. Following the initial phase of the disease, other cells such as macrophages and monocytes mainly mediate the ongoing nonspecific inflammation. At this later stage, auto reactive T cells presumably do not play an important role in sustaining the chronic inflammation. Mechanisms underlying the perpetuation of this cascade of non-specific inflammation are still poorly understood. As a consequence, treatment of the disease until now has been mainly focused on non-specific suppression of inflammation. In patients with chronic arthritis, such juvenile idiopathic arthritis (JIA) and rheumatoid arthritis (RA) blockade of the tumour necrosis factor alpha pathway has proven to be a very potent treatment option. On the other hand, such treatment is costly and fails to induce a long-lasting remission of the disease. As a consequence long-term treatment with immune suppressive agents is necessary, which increases not only the costs of the treatment but also harbours considerable risk for long-term side effects. Thus, the need for additive and/or alternative strategies is growing. Ideally such alternative treatment should be safe, not expensive and specifically modulate cells that are responsible for the inflammation and/or counter-regulation. Lately, a lot of attention has focused on the role of regulatory $T$ cells for the control of autoimmunity. There are currently two well-characterized types of regulatory $T$ cells, $T r 1$ cells and $C D 4^{+} C D 25^{+} T$ regulatory cells. The $\mathrm{CD} 4^{+} \mathrm{CD} 25^{+} \mathrm{T}$ regulatory cells are a heterogeneous group of cells identified by the expression of CD25 and the transcription factor foxP3. We recently showed that these so-called naturally occurring $T$ regulatory cells and regulatory T cells with specificity towards heat shock proteins may play a role in determining disease outcome in JIA [1,2]. Following autologous stem cell transplantation for arthritis T regulatory cell function is restored coinciding with a remission of the arthritis (de Kleer et al., submitted). Moreover, oral treatment with a peptide (dnaJP1), derived from heat shock protein dnal, restored T regulatory cells in peripheral blood mononuclear cells from patients with RA [3].

Thus, due to insufficient numbers of regulatory T cells, feedback mechanisms fail, resulting in an unrestrained proinflammatory immune response and severe tissue damage in RA and JIA. A lack of a counter-regulatory mechanism based by regu- 
latory $\mathrm{T}$ cells is at least in part responsible for the perpetuation of inflammation [4]. Heat shock proteins may be instrumental in restoring the immunological balance and thus contribute to a long-lasting disease remission.

\section{References}

1. de Kleer IM, Wedderburn LR, Taams LS, Patel A, Varsani H, Klein M, de Jager W, Pugayung G, Giannoni F, Rijkers G, et al:: CD4 ${ }^{+}$CD25(bright) regulatory T cells actively regulate inflammation in the joints of patients with the remitting form of juvenile idiopathic arthritis. J Immunol 2004, 172:6435-6443.

2. de Kleer IM, Kamphuis SM, Rijkers GT, Scholtens L, Gordon G, de Jager W, Hafner R, Van De Zee, Van Eden W, Kuis W, Prakken BJ: The spontaneous remission of juvenile idiopathic arthritis is characterized by $\mathrm{CD}^{+} \mathrm{O}^{+} \mathrm{T}$ cells directed to human heat-shock protein 60 capable of producing the regulatory cytokine interleukin-10. Arthritis Rheum 2003, 48:2001-2010.

3. Prakken BJ, Samodal R, Le TD, Giannoni F, Yung GP, Scavulli J, Amox D, Roord S, de Kleer I, Bonnin D, Lanza P, et al:: Epitope-specific immunotherapy induces immune deviation of proinflammatory $\mathrm{T}$ cells in rheumatoid arthritis. Proc Natl Acad Sci USA 2004, 101:4228-4233.

4. Sakaguchi S: Naturally arising $\mathrm{CD4}^{+}$regulatory $t$ cells for immunologic self-tolerance and negative control of immune responses. Annu Rev Immunol 2004, 22:531-562.

S5

\section{Tracking immune responses in vivo}

\section{MK Jenkins}

Center for Immunology, University of Minnesota Medical School, Minneapolis, Minnesota, USA

Arthritis Res Ther 2005, 7(Suppl 1):S5 (DOI 10.1186/ar1510)

We have developed new tools that allow detection of peptide-MHC II presentation to naïve CD4 ${ }^{+} \mathrm{T}$ cells in vivo. These tools were used to track early events in the immune response to a subcutaneously injected antigen. Following such an injection, antigen is quickly carried via lymphatic vessels into the draining lymph nodes, and down through thin collagen-based conduits that run through the T-cell-rich area. Langerhans cells and dermal dendritic cells near the conduits acquired antigen, produced peptide-MHC II complexes, and were the first cells to stimulate naive antigenspecific CD4+ $\mathrm{T}$ cells. About 18 hours after antigen injection, dermal dendritic cells migrated from the injection site and arrived in the lymph nodes displaying a large number of peptide-MHC II complexes. Elimination of these cells truncated CD25 expression by antigen-specific CD4 ${ }^{+} \mathrm{T}$ cells and ablated the development of cellmediated immunity. Injection of antigen with the adjuvant lipopolysaccharide allowed blood-derived myeloid and lymphoid dendritic cells to produce peptide-MHC II complexes. Antigen-specific, but not non-specific, B cells acquired antigen and produced peptide-MHC II complexes by 6 hours. These B cells migrated from random positions in the follicles to the border with the T-cell area, and interacted stably there with peptide-MHC II-specific CD4 ${ }^{+} \mathrm{T}$ cells by about 36 hours. These results demonstrate the antigen-specific $\mathrm{CD} 4^{+} \mathrm{T}$ cells interact with a variety of antigen-presenting cell types during the primary immune response.

S6

Successful approaches in genetics of musculoskeletal diseases MA Brown

Botnar Research Centre, University of Oxford, UK

Arthritis Res Ther 2005, 7(Suppl 1):S6 (DOI 10.1186/ar1511)

Until very recently the field of genetics of musculoskeletal diseases had few successes to report, all essentially limited to monogenic conditions or candidate gene findings mostly of strongly associated genes lying within the MHC. Amongst these success stories have been important contributions to our understanding of conditions such as rheumatoid arthritis and ankylosing spondylitis. However the field stalled on the identification of the polygenes that contribute the majority of the genetic risk in a wide variety of common musculoskeletal diseases. This has led to considerable criticism of the field, with many observers, often critical of the financial costs of this research, arguing that hypothesis-free genetic approaches are fatally flawed and wasteful.

Over the past 5 years, however, an increasing run of genes have been identified using a variety of approaches for conditions including rheumatoid arthritis, ankylosing spondylitis, chondrocalcinosis and osteoporosis. The successful methods that have been applied will be discussed along with illustrations of how these findings have impacted on our understanding of disease pathogenesis.

\section{S7}

Molecular signatures of rheumatoid arthritis characterized by transcriptome and proteome analysis

\section{MO Glocker ${ }^{1}$, J Kekow ${ }^{2}$, H-J Thiesen ${ }^{3}$}

${ }^{1}$ Proteome Center Rostock, University of Rostock, Germany; ${ }^{2}$ Clinic of Rheumatology, University of Magdeburg, Vogelsang, Germany; ${ }^{3}$ Institute of Immunology, University of Rostock, Germany

Arthritis Res Ther 2005, 7(Suppl 1):S7 (DOI 10.1186/ar1513)

Our research is dedicated to the molecular analysis of autoimmune diseases with processes and biomarkers in patients suffering from RA, we searched for conspicuous differences in the abundance of soluble proteins between synovial fluids (SF) and plasmas of patients. Detailed analysis of two-dimensional gel electrophoresis-separated protein spots by means of MALDI-MS and MALDI-QITToF-MS ${ }^{n}$ sequencing $[1,2]$ revealed that the haptoglobin $\alpha$-chain is present in both body fluids in at least four variants. This result suggests that specific posttranslational modification processes occur in SF that could be playing an important role in the inflammatory degradation process of the joint.

We therefore analyzed pannus tissue from RA patients and compared the obtained protein pattern with that from tissues derived from patients with osteoarthritis (OA) first by RNA microarray-analysis (Affymetrix) [3,4]. Our combined approach started with screening the human genome for protease genes. There are 590 proteases encoded by the human genome, 397 of which are found on the Affymetrix chips, represented by 687 unique probe sets. In addition, from the 159 protease inhibitors in the genome, 106 are presented on the chips by 164 unique probe sets. Interestingly, only approximately 330 protease probe sets and approximately 70 inhibitor probe sets yielded in so-called 'present calls'. A comparison of the RNA abundances between RA and OA showed that approximately 40 protease and 16 inhibitor gene products could be identified as differentially expressed. One of them was Cathepsin B, which was found approximately twofold more expressed in the pannus (RA) than in synovial tissue (OA) at the RNA level. Parallel proteomics investigations showed over 800 protein spots in the gels from tissue materials. Approximately 250 of the protein spots were analyzed by mass spectrometry, resulting in the identification of approximately 180 unique proteins. Among the identified proteins 12 belonged to the group of proteases. Again, Cathepsin B was found significantly upregulated. In addition, the small calcium binding protein S100A9 (MRP14) was identified as a discriminatory marker protein in SF by global proteomic analysis $[5,6]$. These results were confirmed by ELISA and it was found that plasma levels of the S100A8/A9 heterocomplex are correlating well with levels in SF; hence determination of plasma levels is applicable for distinguishing RA patients from patients with other inflammatory joint diseases as well as from OA patients and from controls. Initial studies on RA samples indicate that plasma levels of the S100A8/A9 heterocomplex might become a useful marker in monitoring anti-tumor necrosis factor alpha therapy responses.

References

1. Koy C, Mikkat S, Raptakis E, Sutton C, Resch M, Tanaka K, Glocker MO: MALDI-QIT-ToF-MS sequencing resolves structures of unidentified peptides obtained by in-ge/ tryptic digestion of haptoglobin derivatives from human plasma proteomes. Proteomics 2003, 3:851-858.

2. Mikkat s, Koy C, Ulbrich M, Ringel B, Glocker MO: Mass spectrometric protein structure characterization reveals cause of migration differences of haptoglobin $\alpha$-chains in 2-DE gels. Proteomics 2004, 4:3921-3932.

3. Lorenz P, Ruschpler P, Koczan D, Stiehl P, Thiesen H-J: From transcriptome to proteome: differentially expressed proteins identified in synovial tissue of patients suffering from rheumatoid arthritis and osteoarthritis by an initial screen with a panel of 791 antibodies. Proteomics 2003, 3:991-1002.

4. Ruschpler P, Lorenz P, Eichler W, Koczan D, Hänel C, Scholz R, Melzer Ch, Thiesen H-J, Stiehl P: High CXCR3 expression in synovial mast cells associated with CXCL9 and CXCL10 expression in inflammatory synovial tissues of patients with rheumatoid arthritis. Arthritis Res Ther 2003, 5:R241-R252.

5. Ringel B, Drynda S, Schinke I, Mikkat S, Thiesen H-J, Eppert GJ, Kekow J, Glocker MO: Free-flow electrophoresis in combination with HPLC and gel-electrophoresis for the depletion of albumins and enrichment of low-abundant proteins for proteome analysis. In Procedings of the 2003 Congress of the Swiss Proteomics Society: Understanding Biological Systems through Proteomics; 24 December 2003; Basel, Switzerland. Ed. FontisMedia (ISBN 2-88476-004-0):56-57.

6. Drynda S, Ringel B, Kekow M, Kühne C, Drynda A, Glocker MO, Thiesen $\mathrm{H}-\mathrm{J}$, Kekow J: Proteome analysis reveals disease-associated marker proteins to differentiate RA patients from other inflammatory joint diseases with the potenial to monitor anti-TNF- $\alpha$ therapy. Pathol Res Pract 2004, 200:165-171.

S8

Chemokine decoy receptors in inflammation

G Graham

Division of Immunology, Infection and Inflammation, University of Glasgow, UK Arthritis Res Ther 2005, 7(Suppl 1):S8 (DOI 10.1186/ar1711)

\section{S9}

Follow up study of B-lymphocyte depletion in the treatment of patients with systemic lupus erythematosus MJ Leandro, JCW Edwards, MR Ehrenstein, G Cambridge, DA Isenberg Centre for Rheumatology, University College London, London, UK Arthritis Res Ther 2005, 7(Suppl 1):S9 (DOI 10.1186/ar1712)

See page S59 
S10

Mechanisms of cartilage matrix turnover: synergistic interactions of proinflammatory cytokines with oncostatin $M$ in upregulating matrix metalloproteinases and ADAMTS metalloproteinases

TE Cawston, H Bigg, J Milner, J Catterall, T Morgan, E Barksby, W Hui, A Rowan

Rheumatology, School of Clinical Medical Sciences, The Medical School,

University of Newcastle, UK

Arthritis Res Ther 2005, 7(Suppl 1):S10 (DOI 10.1186/ar1516)

Cartilage is a highly organised tissue where the arrangement of collagen and proteoglycan and minor components is carefully controlled. The chondrocytes maintain a precise balance between anabolic and catabolic processes that maintains this extracellular matrix. Proteoglycan removal is rapid and reversible while collagen removal is slower but, once removed, is difficult to replace. Our main research focus is to discover the mechanism of collagen turnover and we study the members of the matrix metalloproteinase (MMP) family that specifically cleave type II collagen to give characteristic one-quarter and three-quarter fragments; MMP-1, MMP-8, MMP-13 and also MMP-2 and MMP-14 cleave in this way.

Oncostatin M (OSM) is a member of the IL-6 family that synergises with IL-1 to induce cartilage proteoglycan and collagen degradation in a cartilage explant culture system [1]. A significant finding is the synergistic induction of the collagenase, MMP-1, which occurs via interplay between the JAK/STAT, AP-1 and MAPK pathways. Other collagenases such as MMP-8 and MMP-13 are also upregulated along with MMP-14 and MMP-3. This latter enzyme can activate the collagenases, and an important feature of OSM may be its ability to promote the activation of enzymes that initiate activation cascades that lead to the production of active collagenases. OSM can also exacerbate the effects of other important proinflammatory mediators such as tumour necrosis factor alpha (TNF- $\alpha$ ) and IL-17.

Molecular and cellular studies aim to discover the mechanism of action that leads to synergy. Affymetrix microarrays show that a specific cohort of genes are upregulated by these cytokine mixtures including MMPs, ADAMs (A disintegrin and metalloproteinases), activators, cell surface proteins and cytokines. Two-dimensional gel electrophoresis and proteomic analysis confirm that many of the corresponding proteins are made by chondrocytes after stimulation. Purification of specific proteins from conditioned culture medium has been undertaken to try and determine the enzymes responsible for collagen turnover.

In order to assess the effects of these cytokine combinations in vivo, we have injected OSM in combination with either IL-1 or TNF- $\alpha$ intra-articularly into murine knee joints using recombinant adenovirus. Engineered adenoviruses were administered for only 7 days, after which time joints were fixed, decalcified and sectioned. Histological analyses indicated marked synovial hyperplasia and inflammatory cell infiltration for IL-1, TNF- $\alpha$ and OSM treated joints but not in controls (joints treated with an 'empty' adenovirus). The inflammation was more pronounced for both OSM $+\mathrm{IL}-1$ and OSM + TNF- $\alpha$ combinations with evidence of extensive cartilage and bone destruction. Significant loss of both proteoglycan and collagen was also seen for these combinations, and an increased expression of MMPs with decreased tissue inhibitors of metalloproteinases was found in both articular cartilage and synovium. The effects of these combinations were significantly greater than those seen with any of the cytokines alone. Cytokine combinations also upregulated RANK/RANKL, and increased the number of TRAP-positive cells showing an increase in osteoclast formation and bone damage. Taken together, these data confirm that, in vivo, OSM can significantly exacerbate the effects of both IL-1 and TNF- $\alpha$ resulting in inflammation and tissue destruction characteristic of that seen in rheumatoid arthritis.

These studies provide further evidence to implicate the upregulation of collagenases as a key factor in the destruction of collagen that occurs in joint pathology, and suggests that OSM is a potent mediator when found in the joint with other proinflammatory cytokines.

Reference

1. Cawston TE, Curry VA, Summers CA, et al.: The role of oncostatin M in animal and human connective tissue collagen turnover and its localization within the rheumatoid joint. Arthritis Rheum 1998, 41:1760-1771.

\section{S11}

Collagenases and aggrecanases: understanding the role of non-catalytic domains in cartilage matrix breakdown H Nagase, R Visse, M Kashiwagi, C Gendron, Y Itoh

Kennedy Institute of Rheumatology Division, Imperial College London, UK

Arthritis Res Ther 2005, 7(Suppl 1):S11 (DOI 10.1186/ar1517)

Degradation of cartilage matrix, which mainly consists of collagen fibrils and aggrecan, seriously impairs the function of joints. The primary cause of this process is elevated proteolytic enzymes. Collagen fibrils are degraded by a group of matrix metalloproteinases (MMPs) including collagenases (MMP-1, MMP-8 and MMP-13), gelatinase A (MMP-2) and MT1-MMP (MMP-14). Aggrecan is degraded by MMPs and 'aggrecanases' belonging to the ADAMTS family. Inhibitors of these metalloproteinases are considered as potential therapeutic agents to protect cartilage degradation, and many active site-directed inhibitors with a zinc-chelating moiety have been developed. Unfortunately, those compounds have serious side effects possibly due to lack of selectivity [1]. In the hope of developing new types of inhibitors we have been investigating the mechanisms of action of collagenases and aggrecanases. Collagenases are unique as they can degrade triple helical interstitial collagens into three-quarter and one-quarter fragments, a crucial step for collagenolysis in the tissue. Typical collagenases consist of a catalytic domain and a hemopexin domain that are connected by a linker peptide. Both domains are essential for collagenolysis. The three-dimensional structure of a prototypic collagenase, collagenase 1 (MMP-1), however, indicated that the active site of the enzyme is too narrow to accommodate triple-helical collagen. We have recently shown that collagenase locally unwinds triple helical collagen before it hydrolyses the peptide bonds [2]. Based on a series of mutagenesis mutagenesis we predict that the collagen binding site is created by both the catalytic and the hemopexin domains. This potential collagen binding groove is partially blocked by the prodomain in the zymogen of MMP-1 (proMMP-1), explaining its inability to bind to collagen unless it is activated. Overall proMMP-1 has a 'closed' configuration in contrast to the 'open' configuration of the active MMP-1. The exact mechanism of how collagenase unwinds collagen is under investigation. Another key collagenase is MT1-MMP. It is highly expressed in rheumatoid synovial lining cells invading into the cartilage. Overexpression of recombinant MT1MMP in COS7 cells in vitro degrade reconstituted type I collagen films. This reaction requires dimerisation of the enzyme on the cell surface through the hemopexin domains. When dimerisation of MT1-MMP is prevented by overexpression of the membrane-anchored MT1-MMP hemopexin domain, the collagenolytic activity was blocked, but not its general photolytic activity.

Among 19 ADAMTSs in humans, six have been shown to have aggrecanase activity. Their non-catalytic domains include one disintegrin, one or more thrombospondin, one cysteine-rich and one spacer domain. Full-length ADAMTS-4 (aggrecanase 1) is active on aggrecan only, but when the C-terminal spacer domain is deleted it expresses broader proteolytic activity and digests fibronectin, fibromodulin, decorin and biglycan, as well as maintaining aggrecanase activity [3]. Further deletion of the cysteine-rich and thrombospondin domains greatly reduces both activities [3].

These studies suggest that activities of collagenases and aggrecanases will be attenuated by inhibitors or antibodies that interact directly with their non-catalytic ancillary domains (exosite/allosteric inhibitors). Such molecules will be attractive for therapy as they will be highly selective because they are based on the unique mechanism of each proteinase.

References

1. Peterson JT: Matrix metalloproteinase inhibitor development and the remodeling of drug discovery. Heart Fail Rev 2004, 9:63-79.

2. Chung L, Dinakarpandian D, Yoshida N, Lauer-Fields JL, Fields GB, Visse $\mathrm{R}$, Nagase $\mathrm{H}$ : Collagenase unwinds triple-helical collagen prior to peptide bond hydrolysis. EMBO J 2004, 23:3020-3030.

3. Kashiwagi M, Enghild JJ, Gendron C, Hughes C, Caterson B, Itoh Y, Nagase $\mathrm{H}$ : Altered proteolytic activities of ADAMTS-4 expressed by Cterminal processing. J Biol Chem 2004, 279:10109-10119.

Acknowledgements This work was supported by grants from the Wellcome Trust, Cancer Research UK, the Arthritis Research Campaign and the Nation Institutes of Health (USA).

\section{S12}

\section{The effects of inflammatory arthritis on bone remodeling SR Goldring}

Beth Israel Deaconess Medical Center, Division of Rheumatology, and New England Baptist Bone \& Joint Institute, Harvard Institutes of Medicine, Boston, Massachusetts, USA

Arthritis Res Ther 2005, 7(Suppl 1):S12 (DOI 10.1186/ar1518)

Rheumatoid arthritis $(R A)$ represents an excellent model for gaining insights into the adverse effects of inflammatory arthritis on skeletal remodeling. Bone erosions at the margins of diarthrodial joints represent the radiographic hallmark of RA. Histopathologic examination of the bone-pannus interface in patients with RA reveals the presence of a heterogeneous population of cells lining the bone surface, including multinucleated cells expressing the full phenotypic repertoire of osteoclasts, the cells that mediate bone resorption in physiologic remodeling. Further evidence implicating osteoclasts in the pathogenesis of marginal joint erosions is derived from animal models of inflammatory arthritis in which animals lacking the capacity to form osteoclasts fail to develop joint erosions. Studies have shown that the RA synovial tissue contains abundant osteoclast precursors and that the RA synovium is a rich source of factors with potent osteoclast differentiation and activation activity. Particular attention has focused on receptor activator of NF-KB ligand (RANKL), a member of the tumor necrosis factor ligand family, because of the requirement of this factor for osteoclastogenesis. RANKL is expressed by synovial fibroblasts and activated T cells in RA synovial tissues and in several different animal models of inflammatory arthritis, treatment with osteoprotegerin (the soluble receptor that inhibits RANKL activity) results in marked suppression of focal bone erosions. In addition, mice possessing the null mutation for RANKL are protected from focal bone destruction in the serum transfer model of inflammatory arthritis. An additional observation in patients with active RA is the absence of radiographic evidence of bone repair. This finding suggests that the processes that regulate coupling of bone resorption and formation under physiologic conditions have been disrupted and that the enhanced focal bone 
resorption is not matched by a compensatory increase in bone formation. In addition to the disordered focal bone remodeling associated with the synovial lesion, patients with RA also exhibit evidence of generalized axial and appendicular bone loss at sites that are distant from inflamed joints. Assessment of biochemical markers of bone turnover indicates that there is a generalized increase in bone resorption, and that there is a correlation between disease activity and the rate of systemic bone resorption. It is likely that this generalized skeletal bone loss is mediated by synovial cytokines with osteoclastogenic activity that enter the circulation and act as 'endocrine' factors to adversely affect systemic bone remodeling. Recent studies indicate that agents, such as the bisphosphonates, that target osteoclast-mediated bone resorption have the capacity to reduce not only generalized bone osteoporosis but also may have efficacy in retarding the progression of focal joint erosions.

\section{S13}

\section{Genetics and bone disease \\ SH Ralston}

Rheumatic Diseases Unit, University of Edinburgh, UK

Arthritis Res Ther 2005, 7(Suppl 1):S13 (DOI 10.1186/ar1519)

Bone diseases are a common cause of morbidity and mortality in developed countries, and genetic factors play an important role in the pathogenesis of these diseases. The most common form of bone disease is osteoporosis, which is characterized by reduced bone mineral density (BMD) and an increased risk of fracture. Environmental factors such as diet and exercise influence BMD but genetic factors play a predominant role, accounting for up to $85 \%$ of the variation in peak BMD. Several candidate genes have been identified that regulate BMD and susceptibility to fracture, including bone morphogenic protein 2, collagen type I alpha 1, the vitamin $\mathrm{D}$ receptor, the estrogen receptor and lipoprotein receptor related protein 5 (LRP5). Paget's disease of the bone (PDB) is characterised by focal abnormalities of increased bone turnover. Mutations in several genes have been identified as causes of PDB and related syndromes including receptor activator of NF-KB (RANK), osteoprotegerin, sequestosome 1 and valosin containing protein. All of these genes play a role in the RANK signalling pathway, which is essential for osteoclast activation. Rare bone diseases such as osteopetrosis and hereditary osteoscleroses are also caused by mutations in genes that affect bone cell function. Osteopetrosis is characterised by increased $\mathrm{BMD}$, and failure of osteoclastic bone resorption due to mutations in genes that encode proteins that are essential for osteoclast activity like the chloride pump and proton pump, or mutations in genes like cathepsin $\mathrm{K}$, which breakdown bone matrix. Although the bones are dense, fractures are common in osteopetrosis because of reduced bone quality. Osteosclerosis occurs because of mutations in genes that increase osteoblast activity, including SOST, transforming growth factor beta and LRP5. Osteosclerotic patients also have increased BMD but fractures are rare, because bone quality is normal. From a clinical standpoint, advances in knowledge about the genetic basis of bone disease offers the prospect of developing new markers for assessing fracture risk and the identification of new molecular targets that will form the basis for the design of new treatments.

\section{S14}

\section{Defining a role for fibroblasts in the persistence of chronic inflammatory joint disease}

\section{Buckley, A Filer, O Haworth, G Parsonage, K Raza, M Salmon}

Rheumatology Research Group, Division of Immunity and Infection, MRC Centre for Immune Regulation, The University of Birmingham, UK

Arthritis Res Ther 2005, 7(Suppl 1):S14 (DOI 10.1186/ar1512)

One of the most important but as yet unanswered questions in inflammation research is not why chronic inflammation occurs but why it does not resolve. Current models of inflammation stress the role of antigen-specific lymphocyte responses and attempt to address the causative agent. However, recent studies have begun to challenge the primacy of the lymphocyte and have begun to focus on an extended immune system in which stromal cells, such as macrophages and fibroblasts, play a role in the persistence of the inflammatory lesion.

In this lecture I will illustrate how fibroblasts play an important role in regulating the switch from acute resolving to chronic persistent inflammation associated with the pathology of diseases such as rheumatoid arthritis [1]. In chronic inflammation the normal physiological process of the death and emigration of unwanted inflammatory effector cells becomes disordered leading to accumulation of leucocytes [2-4] within lymphoid aggregates that resemble those seen in lymphoid tissue [5]. I will describe how fibroblasts from the rheumatoid joint provide survival and retention signals for leucocytes leading to their inappropriate and persistent accumulation within inflamed tissue [6]. Our work suggests that targeting the stromal microenvironment is likely to be an important strategy for future anti-inflammatory therapies.

\section{References}

1. Buckley CD, Pilling D, Lord JM, Akbar AN, Scheel-Toellner D, Salmon M: Fibroblasts regulate the switch from acute resolving to chronic persistent inflammation. Trends Immuno/ 2001, 22:199-204.

2. Salmon $M$, Scheel-Toellner D, Huissoon AP, Pilling D, Shamsadeen N, Hyde H, D'Angeac AD, Bacon PA, Emery P, Akbar AN: Inhibition of T cell apoptosis in the rheumatoid synovium. J Clin Invest 1997, 99:439-446.
F, Amara A, Curnow SJ, Lord JM, Scheel-Toellner D, Salmon M: Persistent induction of the chemokine receptor CXCR4 by TGF-beta 1 on synovial $T$ cells contributes to their accumulation within the rheumatoid synovium. J Immuno/ 2000, 165:3423-3429.

4. Buckley CD: Why do leucocytes accumulate within chronically inflamed joints? Rheumatology 2003, 42:1433-1444.

5. Amft N, Curnow SJ, Scheel-Toellner D, Devadas A, Oates J, Crocker J, Hamburger J, Ainsworth J, Mathews J, Salmon M, et al.: Ectopic expression of the $B$ cell attracting chemokine BCA-1 (CXCL13) on endothelial cells and within lymphoid follicles contributes to the establishment of germinal center-like structures in Sjögren's Syndrome. Arthritis Rheum 2001, 44:2633-2641.

6. Parsonage G, Falciani F, Burman A, Filer A, Ross E, Bofill M, Martin S, Salmon M, Buckley CD: Global gene expression profiles in fibroblasts from synovial, skin and lymphoid tissue reveals distinct cytokine and chemokine expression patterns. Thromb Haemost 2003, 90:688-697.

\section{S15}

\section{Regulating apoptosis in fibroblasts}

T Pap', A Cinski², A Drynda², A Wille², A Baier², S Gay³, I Meinecke ${ }^{4}$

${ }^{1}$ Division of Molecular Medicine of Musculoskeletal Tissue, University Hospital, Munster, Germany; ${ }^{2}$ Division of Experimental Rheumatology, University Hospital Magdeburg, Germany; ${ }^{3}$ Center of Experimental Rheumatology, Department of Rheumatology, University Hospital, Zurich, Switzerland; ${ }^{4}$ Department of Traumatology, Hand and Reconstructive Surgery, University Hospital, Munster, Germany Arthritis Res Ther 2005, 7(Suppl 1):S15 (DOI 10.1186/ar1515)

Apoptosis constitutes a highly selective way of eliminating aged and injured cells and is a key mechanism for the balanced growth and regeneration of tissues. In addition to genotoxic stress and the withdrawal of growth factors, apoptosis can be induced by death receptors. Such receptors trigger apoptosis through an approximately 90 amino acid death domain. Prominent and best-characterized members of the death domain receptor family are Fas (CD-95/Apo-1) and the p55 TNF receptor (TNFRI). Alterations in receptor-mediated apoptosis may result in changes of tissue homeostasis and have been found in a variety of malignancies as well as in the inflamed and hyperplastic synovium in rheumatoid arthritis. Accumulating evidence suggests that the activation of rheumatoid arthritis synovial fibroblasts (RASF) is associated with alterations in apoptosis, especially at sites of invasion into cartilage and bone. Specifically, it has been demonstrated that RASF are less susceptible to Fas-induced cell death than osteoarthritis synovial fibroblasts but show higher expression levels of Fas.

Recent studies have shown that both cytokine-dependent and cytokine-independent mechanisms contribute to the resistance of RASF against Fas-induced apoptosis. Thus, tumor necrosis factor alpha (TNF- $\alpha$ ) - a major inflammatory cytokine in the rheumatoid synovium - fails to induce apoptosis in RASF, but reduces the susceptibility of these cells to Fas-mediated cell death through the induction of the transcription factor NF- $\kappa B$. Providing a link between altered apoptosis and cartilage destruction, we have shown that overexpression of TIMP-3 through gene transfer not only reduces the invasiveness of RASF but also modulates the apoptosis-inhibiting effects of TNF- $\alpha$. RASF overexpressing TIMP-3 are sensitized strongly to Fas/CD95-mediated cell death by TNF- $\alpha$, and gene transfer of TIMP-3 inhibits the TNF- $\alpha$-induced activation of NF- $\mathrm{KB}$. While these effects of TNF- $\alpha$ on apoptosis can be found in different fibroblasts, several anti-apoptotic molecules have been demonstrated to be upregulated specifically in RASF.

In this context, we have investigated the role of the small ubiquitin-like modifier SUMO-1 in the regulation of apoptosis. We found that increased levels of SUMO-1 in RASF but not in osteoarthritis synovial fibroblasts were associated with a reduced susceptibility of RASF to Fas-induced apoptosis. Using small interfering (si)RNA to knock-down the expression of SUMO-1 as well as retroviral gene transfer of SUMO-1, we could establish a functional relationship between the expression of SUMO-1 and the resistance of RASF against apoptosis. Moreover, it was demonstrated by gene transfer of the nuclear SUMO-protease SENP1 that, rather than by interacting directly with the Fas-associated death domain, SUMO-1 inhibits apoptosis through recruiting pro-apoptotic molecules such as DAXX into nuclear PML bodies, where they cannot exert their pro-apoptotic effects.

Taken together, there is growing evidence that in activated fibroblasts, such as in RASF, there is a close association between anti-apoptotic and destructive pathways. In addition to cytokine-dependent inflammatory mechanisms, the intrinsic upregulation of anti-apoptotic molecules contributes to the resistance of RASF against apoptosis. Post-translational modification of nuclear proteins through SUMO-1 appears to constitute an important mechanism of apoptosis regulation. Therefore, the inhibition of molecules that confer the resistance of RASF to apoptosis constitutes a most interesting therapeutic target. 
S16

Innate immunity and the anti-viral state of rheumatoid synovium

\section{GS Firestein}

UCSD School of Medicine, La Jolla, California, USA

Arthritis Res Ther 2005, 7(Suppl 1):S16 (DOI 10.1186/ar1521)

Rheumatoid arthritis (RA) is an immune-mediated disease with synovial inflammation and invasion of the extracellular matrix. While adaptive immunity plays a key role in the pathogenesis of RA, the contribution of innate immune responses has been increasingly appreciated in recent years. Toll-like receptors (TLRs) and cytokines in the rheumatoid synovium can activate signal transduction pathways that induce proinflammatory genes and perpetuate synovitis. Understanding these signaling mechanisms helps us understand the pathogenesis of disease and identify novel therapeutic targets.

Among the signaling pathways that are potentially involved in inflammation, the IKK-related kinase, inducible IKK (IKKi or IKKE), appears to play a key role in innate immune responses in the joint and modulates synovial inflammatory responses. This kinase, along with a second IKK-related kinase TANK binding kinase 1 (TBK1), was originally identified as a NF-KB activating enzyme that phosphorylates IkB. It is now clear, however, that this represents only one of several substrates for this family. For instance, IKKi also phosphorylates interferon regulatory factor (IRF) and coordinates the activation of IRF and NF-KB after TLR ligation. IKKi may also link the NF-KB and CCAAT enhancer binding protein (C/EBP) pathways in lipopolysaccharide-stimulated cells. The novel roles for IKKi suggests that it contributes to the signaling pathways involved in synovial inflammation. Moreover, IKKi appears to play a key role in the establishment of an 'antiviral state' through the activation of IRF3 and c-Jun with subsequent production of interferon beta (IFN- $\beta$ ) and other genes involved in host defense.

We previously demonstrated that IKKi is constitutively expressed in RA synovial tissue and fibroblast-like synoviocytes (FLS), and that the gene can be induced by cytokine stimulation. More recent studies now show that functional activity of IKKi in cultured synoviocytes is rapidly increased by exposure to proinflammatory cytokines and TLR agonists. We have also shown that IRF3 is activated in RA synovium, which represents an early step in the IKKi-mediated expression of antiviral genes like IFN- $\beta$. Additional preliminary data with human FLS suggest that the IKKi pathway can activate other signaling cascades, such as c-Jun, which play a role in assembly of the interferon ehanceosome to increase IFN- $\beta$ expression. Using FLS from IKKi knockout mice, we have shown that IKKi can act as a key link between TLR/cytokine receptor ligation and expression of cytokines and matrix metalloproteinases. IKKi might serve in an alternative signaling pathway for activation of innate immunity in RA and establishing an anti-viral state in the synovium, especially through the activation of IRF3-driven and c-Jun-driven genes.

\section{S17}

\section{Signal transduction in T lymphocytes}

D Cantrell

Division of Cell Biology \& Immunology, School of Life Sciences, University of Dundee, Dundee, UK

Arthritis Res Ther 2005, 7(Suppl 1):S17 (DOI 10.1186/ar1520)

\section{S18}

\section{Title to be confirmed}

\section{J Kalden}

University Erlangen-Nuremberg, Erlangen, Germany

Arthritis Res Ther 2005, 7(Suppl 1):S18 (DOI 10.1186/ar1713)

\section{Poster abstracts}

\section{P1}

Differential effects of T-cell specific PKC-theta inhibition or selective intra-articular NF- $\kappa$ B inhibition on synovial inflammation in rat adjuvant arthritis

\section{SW Tas', MJ Vervoordeldonk', N Hajji', M May², S Ghosh'3 ${ }^{3}$ PP Tak ${ }^{1}$}

${ }^{1}$ Division of Clinical Immunology \& Rheumatology, AMC/University of Amsterdam, The Netherlands; ${ }^{2}$ School of Veterinary Medicine, Department of Animal Biology, University of Pennsylvania, Philadelphia, Pennsylvania, USA; ${ }^{3} / m m u n o b i o l o g y$ Section, Yale University Medical School, New Haven, Connecticut, USA Arthritis Res Ther 2005, 7(Suppl 1):P1 (DOl 10.1186/ar1522)

Objective NF- $\mathrm{KB}$ is a key regulator of synovial inflammation. In the initiation phase of rheumatoid arthritis (RA) dendritic cells and $\mathrm{T}$ cells are likely to be important, whereas in established arthritis other cells play a key role as well. We investigated the relative effect of upstream NF-kB inhibition specifically in T cells versus non-cell type specific NF-KB inhibition in different stages of rat adjuvant arthritis (AA), using either the T-cell specific PKC-theta inhibitory factor (PIF) peptides or non-cell type specific IKK-beta blocking NEMO binding domain (NBD) peptides. The effects of the NBD peptide on human RA synovial tissue in culture were also evaluated. Methods AA was induced in Lewis rats by intradermal injection of heat-killed mycobacteria. Rats develop clinical signs of arthritis 10-12 days after immunization. PIF or NBD peptides $(150 \mu \mathrm{g})$ were administered either intraperitoneally or intra-articularly into the ankle joint at different time-points. The course and severity of arthritis was monitored using water-displacement plethysmometry. On day 21 rats were sacrificed and tissue specimens were collected for routine histology and X-rays of the ankle joints. Human RA synovial tissue was collected by arthroscopy and cultured ex vivo \pm NBD $(100 \mu \mathrm{M})$. Tumor necrosis factor alpha induced IL- 6 production was measured in the supernatant after 7 days by ELISA. Results Intra-articular injection of the T-cell specific PKC-theta inhibitor PIF on days 10 and 12 did not result in amelioration of arthritis nor reduced bone erosion. However, intraperitoneal injection of PIF on days $-1,1$ and 3 resulted in reduced T-cell proliferation on day 5 in response to TCR triggering $(50 \%$; $P=0.03)$ or cognate antigen (PPD) $(30 \% ; P=0.05)$. Furthermore, this resulted in a slightly reduced severity of arthritis (area under the curve 18.47 versus 22.86; $P=$ not significant) and radiological damage (erosion score 4 versus 6 ; $P=$ not significant). Non-cell type specific NF-KB blockade by the NBD peptide resulted, however, in significantly reduced severity of arthritis $(P<0.0001)$ and radiological damage $(P=0.04)$ when injected intra-articularly on days 10 and 12 . In addition, proinflammatory cytokine expression was significantly lower in synovial tissue of NBD-treated rats. Incubation of human RA synovial tissue with NBD peptides resulted in $33 \%$ inhibition of tumor necrosis factor alpha induced IL-6 production in the supernatant $(P<0.01)$.

Conclusions Specific NF-KB blockade using a small molecule inhibitor of IKK beta has anti-inflammatory effects in AA and human RA synovial tissue. Inhibition of PKC-theta has clear effects on T-cell proliferation when administered around the induction of arthritis, but very limited effects on the clinical course of arthritis, suggesting that NF-KB activity in other cell types contributes significantly to the inflammatory response. These results indicate that IKK-beta-targeted NF-KB blockade is superior to T-cell specific PKC-theta inhibition in established arthritis. Acknowledgement This study was supported by a EULAR Young Investigator Award to SWT.

P2

Positive cytoplasmic indirect immunofluorescence on HEp2 cells of unknown pattern: what to report to rheumatologists Z Rotar, B Boziè, B Rozman, T Kveder

University Medical Centre, Department of Rheumatology, Ljubljana, Slovenia Arthritis Res Ther 2005, 7(Suppl 1):P2 (DOI 10.1186/ar1523)

The aim of our study was to evaluate antibodies against 'specific liver' antigens in selected sera from patients with positive 'AMA like' and unidentified (UD) Hep2 cytoplasmic immunofluorescence (ctp IIF).

Sera of 75 inpatients and outpatients from the Department of Rheumatology, Ljubljana met the inclusion criteria: 36 with AMA-like and 39 with UD pattern. All sera were also negative for anti-M2 and/or antibodies against pyruvat dehydrogenase complex as well as negative to other defined ctp IIF patterns according to European Consensus Finding Study: lysosomal-like, Golgi-like, vimentin-like and actin-like. The majority of patients fulfilled the criteria for connective tissue diseases and rheumatoid arthritis.

Antibodies against intracellular antigens were screened by IIF on HEp2 cells (Immunoconcepts, Sacramento, CA, USA). Antibodies against LKM1, SLA, LC1 and Sp100 were performed by line immunoassay (Imtec, Berlin, Germany).

There was no difference in AMA-like and UD ctp IIF concerning antibodies against 'specific liver' antigens. A surprisingly high prevalence of positive antiLKM1 was found in sera regardless of the IIF pattern (Table 1)

Table 1

Antibodies against 'liver specific' antigens in sera with AMA-like and UD ctp IIF

\begin{tabular}{lccccc}
\hline & $n$ & Sp100 & LKM1 & SLA & LC1 \\
\hline AMA-like & 42 & 4 & $19(45 \%)$ & 0 & 1 \\
Unidentified & 33 & 4 & $14(42 \%)$ & 2 & 0 \\
All & 75 & $8(11 \%)$ & $33(44 \%)$ & $2(3 \%)$ & $1(1 \%)$ \\
\hline
\end{tabular}

Anti-LKM1 has been considered as one of the markers for autoimmune hepatitis type II characterised by fulminant onset and high inflammatory activity according to Imtec data. None of our patients with positive anti-LKM1 had any serious 
hepatic involvement, therefore they had not been histopathologically evaluated. Only few of them exhibited minor deviations in liver enzymes (less than $1 \times$ upper normal limit). There was slightly lower prevalence of connective tissue disease patients found among UD ctp IIF (Table 2).

\section{Table 2}

\begin{tabular}{|c|c|c|c|c|c|}
\hline Patients & $n(\%)$ & Sp100 & LKM1 & SLA & LC1 \\
\hline \multicolumn{6}{|l|}{ AMA $(n=42)$} \\
\hline $\begin{array}{l}\text { Connective tissue } \\
\text { diseases }\end{array}$ & $25 / 42(60 \%)$ & 3 & 8 & 0 & 1 \\
\hline Rheumatoid arthritis & $6 / 42(14 \%)$ & 0 & 5 & 0 & 0 \\
\hline Other & $11 / 42(26 \%)$ & 1 & 6 & 0 & 0 \\
\hline \multicolumn{6}{|l|}{ Unidentified $(n=33)$} \\
\hline $\begin{array}{l}\text { Connective tissue } \\
\text { diseases }\end{array}$ & $15 / 33(46 \%)$ & 3 & 6 & 2 & 0 \\
\hline Rheumatoid arthritis & $6 / 33(18 \%)$ & 0 & 2 & 0 & 0 \\
\hline Other & $12 / 33(36 \%)$ & 1 & 6 & 0 & 0 \\
\hline
\end{tabular}

An UD ctp IIF pattern is a frequent laboratory finding but it seems not to be of much help to rheumatologists. A search for antibodies against 'specific liver' antigens seems not to be justified for delineation of hepatic involvement in patients with rheumatic diseases. According to the literature in patients with autoimmune hepatitis the meaning of these antibodies is different.

\section{P3}

\section{Synovial hypoxia inducible factor- $1 \alpha$ expression is inversely related to tissue oxygen levels in vivo in inflammatory arthritis \\ P Donatien'1, V Sandhu'2, D Peston², A Sandisson'2, PC Taylor ${ }^{1,2}$ \\ ${ }^{1}$ Kennedy Institute of Rheumatology Division, Imperial College, London, UK; ${ }^{2}$ Charing Cross Hospital, London, UK \\ Arthritis Res Ther 2005, 7(Suppl 1):P3 (DOI 10.1186/ar1524)}

Background Tissue hypoxia in inflammation induces the upregulation of a gene program associated with angiogenesis, glycolysis and adaptation to $\mathrm{pH}$ via the hypoxia-inducible transcription factors (HIFs). In this study we investigate the relationship between synovial tissue oxygen levels in inflammatory arthritis and tissue expression of HIF-1 $\alpha$ and vascular endothelial growth factor (VEGF).

Methods Novel silver microelectrode technology was used to measure synovial tissue oxygen levels under direct vision during arthroscopy in six patients with inflammatory arthritis of the knee. A sterile silver needle electrode comprising a teflon-insulated silver wire was embedded in a $19 \mathrm{G}$ hypodermic needle of $10 \mathrm{~cm}$ length and introduced to the intra-articular space via a medial suprapatellar approach such that the electrode tip could be directly visualised by the arthroscope introduced from a standard lateral infrapatellar approach. The voltage applied was adjusted to give a diffusion-limited current for the reduction of oxygen. Synovial biopsies were taken under direct vision from exactly the point at which tissue oxygen levels were measured and snap-frozen immediately. HIF-1 $\alpha$, HIF-2 $\alpha$ and VEGF were detected by immunohistochemistry in fixed synovial biopsy sections and the immunopositive area fraction was determined by quantitative digital image analysis.

Results The mean and standard deviation for oxygen tension in the synovial tissue of the joints sampled was $15.4 \pm 9.6 \mathrm{mmHg}$, range $24.4 \mathrm{mmHg}$. The mean and standard deviation for immunopositive area fraction for HIF-1 $\alpha$ was $0.64 \pm 0.39 \%$ and for HIF- $2 \alpha$ was $4.9 \pm 6.7 \%$. HIF- $1 \alpha$ and HIF- $2 \alpha$ staining were detected predominantly in the synovial lining layer. There was a significant, inverse relationship between synovial tissue oxygen measurement in vivo and tissue expression of HIF-1 (Pearson $r=-0.86, P=0.03$ ) and similarly between synovial tissue oxygen and tissue expression of VEGF (Pearson $r=-0.89$, $P=0.02$ ). There was a positive, highly significant correlation between synovial expression of HIF-1 $\alpha$ and VEGF (Pearson $r=0.97, P=0.0005$ ).

Conclusions In this study we directly demonstrate that intra-articular $\mathrm{pO}_{2}$ is markedly reduced in inflammatory arthritis. Furthermore, in the same patients, synovial tissue oxygen tensions are inversely proportional to HIF-1 $\alpha$ and VEGF expression, suggesting that this environment contributes to the perpetuation of inflammation and associated tissue destruction.
P4

Prophylactic administration of abatacept (CTLA4-Ig; BMS-188667) prevents disease induction and bone destruction in a rat model of collagen-induced arthritis C Kliwinski, D Kukral, J Postelnek, B Krishnan, L Killar, S Nadler, R Townsend Bristol-Myers Squibb Pharmaceutical Research Institute, Princeton, New Jersey, USA Arthritis Res Ther 2005, 7(Suppl 1):P4 (DOI 10.1186/ar1525)

Background and objectives Rheumatoid arthritis is an autoreactive disease in which activated $T$ cells play an important role orchestrating the autoimmune responses giving rise to the inflammatory cascade responsible for joint inflammation and bone destruction. The CD28/B7 costimulatory pathway is critical for full T-cell activation, and modulating this pathway has been shown to inhibit T-cell activation leading to inhibition of these immune responses. Abatacept modulates T-cell activation by interfering with the engagement of CD80/86 with CD28. Abatacept has been shown to provide significant improvement in the signs and symptoms of rheumatoid arthritis in a phase II trial. Here, we examine the effect abatacept administration has on disease induction, anti-collagen antibody production and bone destruction in a rat model of collagen-induced arthritis.

Methods Female DA rats were immunized subcutaneously on day 0 with $300 \mu \mathrm{g}$ bovine type II collagen in incomplete Freund's adjuvant at the base of the tail. Immunized rats were administered either $1 \mathrm{mg} / \mathrm{kg}$ abatacept or a control human $\lg$ IP on days $-1,0,2,4,6,8$ and 10 . Disease progression was monitored by measuring the paw volume in milliliters with a plethysmometer. Both hind paws were measured and the change in volume from baseline measurements (day 0 ) were recorded. At the conclusion of the study (day 27) serum samples were collected from each animal for measurement of collagen-specific antibodies by ELISA as well as serum cytokine measurements. Legs from the rats were removed and placed in formalin and prepared for histological analysis as well as analysis of bone morphology by micro-CT.

Results By day 16 of the study, significant paw swelling was observed in the IgG-treated control animals and continued to increase throughout the study until reaching a plateau $(\sim 3-3.5 \mathrm{ml})$ on day 21 . Administration of abatacept abrogated paw swelling throughout the course of the study. The IgG-treated rats reached $100 \%$ incidence while no incidence was observed in the abatacepttreated group. Serum anti-collagen antibody levels correlated well with the paw swelling data where abatacept administration resulted in $90 \%$ inhibition of collagen-specific antibodies. We also found that abatacept decreased the expression of many of the circulating cytokines and chemokines that were upregulated in diseased animals. The micro-CT data revealed that abatacept treatment protects the bone from destruction as the knees and ankles of these rats appear to be normal. Conclusion Abatacept, a selective co-stimulation modulator, significantly inhibited the onset and progression of disease in a rat collagen-induced arthritis model. In these studies, paw swelling, collagen-specific antibodies and bone destruction were all inhibited by the treatment.

\section{P5}

\section{The transcription T-bet is required for optimal proinflammatory trafficking of $\mathrm{CD4}^{+} \mathrm{T}$ cells RM Rao', GM Lord'2, H Choe', AH Lichtman', FW Luscinskas', LH Glimcher ${ }^{2}$}

${ }^{1}$ Center of Excellence in Vascular Biology, Department of Pathology, Brigham and Women's Hospital, Boston, Massachusetts, USA; 2Department of Immunology and Infectious Diseases, Harvard School of Public Health, Boston, Massachusetts, USA; ${ }^{3}$ Department of Pediatrics, Children's Hospital, Boston, Massachusetts, USA Arthritis Res Ther 2005, 7(Suppl 1):P5 (DOI 10.1186/ar1526)

Background The transcription factor T-bet is a critical regulator of Th1 effector function. Animals deficient in T-bet are protected from a variety of inflammatory diseases, including systemic lupus erythematosus and inflammatory arthritis. An essential function of Th1 cells is the ability to traffic appropriately to sites of inflammation, which is largely dependent on the expression of specific selectin ligands and chemokine receptors. We therefore hypothesised that T-bet would modulate lymphocyte trafficking in vitro and in vivo by direct regulation of both selectin binding and chemokine function.

Methods Balb/c mice deficient in, or transgenic for, T-bet had been generated previously. T-bet ${ }^{-1-} \times \mathrm{DO} 11.10 \mathrm{TCR}$ and $\mathrm{T}-\mathrm{bet}^{-1-} \times \mathrm{IFN}^{-1-}$ mice were generated by backcrossing for $>10$ generations. CD4 ${ }^{+} \mathrm{T}$ cells were generated from primary lymph nodes from all these mice by positive selection and stimulation with appropriate antigen. Functional analysis used the following four methods: adoptive transfer into WT Balb/c mice, which were then injected with OVA, cells were harvested from the spleen, lymph node and peritoneum; selectin binding, interactions with immoblised P-selectin and E-selectin under conditions of laminar flow were examined in a parallel plate flow chamber; expression of selectin ligands, using flow cytometry, real-time $\mathrm{PCR}$ and ${ }^{35} \mathrm{~S}$ incorporation; and chemokine receptor expression and function, using flow cytometry, real-time PCR, transwell chemotaxis and endothelial binding under flow conditions. 


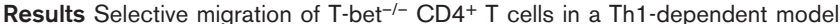
of peritoneal inflammation was completely abrogated. Further investigation revealed that this effect was due to a $50 \%$ reduction in binding to P-selectin but not E-selectin under in vitro flow conditions and that this was as a result of impaired tyrosine sulfation of PSGL-1. In addition, mRNA and surface expression of CXCR3, but not CCR5, was reduced and this was associated with a reduction in both transwell chemotaxis and binding to endothelial cells. Retroviral transfer experiments of T-bet cDNA into T-bet ${ }^{-/-}$and $\mathrm{T}^{- \text {bet }^{-/-}} \times \mathrm{IFN}^{-/-}$cells demonstrated that these effects were independent of interferon.

Conclusions These data establish that T-bet imprints a specific migratory program onto developing $\mathrm{CD}^{+}$cells via control of PSGL-1 sulfation (and thus P-selectin binding) and CXCR3 expression and function. Furthermore, as E-selectin and CCR5 binding are unimpaired, this reveals a level of control on trafficking of Th1 lymphocytes not recognised by previous paradigms.

Acknowledgements This work was supported by the Arthritis Research Campaign (R0600), the Medical Research Council (G108/380) and the National Institutes of Health (Al48126, HL36028, HL53993).

\section{P6}

\section{Specificity of CD4 T-cell responses to aggrecan in BALB/c and DR1 transgenic mice}

K Lowes', AA von Delwig', N McKie', DM Altmann², JA Goodacre ${ }^{3}$, AD Rowan', JH Robinson'

${ }^{1}$ Musculoskeletal Research Group, Clinical Medical Sciences, University of Newcastle upon Tyne, UK; ${ }^{2}$ Human Disease Immunogenetics Group, Imperial College School of Medicine, London, UK; ${ }^{3}$ Lancashire Postgraduate School of Medicine and Health, Preston, UK

Arthritis Res Ther 2005, 7(Suppl 1):P6 (DOI 10.1186/ar1527)

Background It has been suggested that rheumatoid arthritis (RA) is initiated or perpetuated by CD4 T cells activated by presentation of joint antigens by susceptible MHC class II molecules including HLA-DR4 and DR1. A number of candidate antigens have been investigated including cartilage-derived type II collagen and aggrecan. Immunisation of susceptible strains of mice with aggrecan, including BALB/c $\left(\mathrm{H}-2^{\mathrm{d}}\right)$, induces autoimmune arthritis, providing an animal model of human RA [1]. Immunisation with peptides predicted to bind to HLADR4 also induces T-cell responses in HLA-DR4 transgenic mice [2]. The autoimmune $\mathrm{T}$-cell response focuses on only a few epitopes within restricted regions of aggrecan such as the $G_{1}$ globular domain, for reasons that are poorly understood. One explanation is a failure of self-tolerance due to poor presentation of these aggrecan epitopes in the thymus, and presentation of the same epitopes in joints following release and processing of the normally sequestered aggrecan induced by early inflammatory events. T-cell responses have also been observed in RA patients mapping epitopes within the $G_{1}$ domain.

Aim To characterise aggrecan-specific T-cell hybridomas from BALB/c ( $\left.\mathrm{H}-2^{\mathrm{d}}\right)$ and DR1 transgenic mice to investigate the mechanisms of antigen presentation of arthritogenic CD4 T-cell epitopes of aggrecan.

Methods Aggrecan-specific T-cell hybridomas were generated from spleen cells of BALB/c and DRB1*0101-transgenic mouse-MHC class II knockout (DR1-tg) mice immunised with deglycosylated bovine aggrecan. Synthetic peptides of three known arthritogenic $\mathrm{H}-2^{\mathrm{d}}$-restricted epitopes (amino acids 68-82, 84-103 and 169-189) and three DR1-restricted epitopes known to be recognised by RA patients (amino acids $148-165,201-213$ and $292-311$ ) from the $G_{1}$ domain of bovine aggrecan were used to define the specificity of T-cell hybridomas. Cultured bone marrow macrophages were treated with deglycosylated bovine aggrecan or synthetic peptides and used as antigen-presenting cells. T-cell hybridoma responses were measured by proliferation of CTLL-2 indicator cells to T-cell hybridoma supernatants in the presence of tritiated thymidine and expressed as counts per minute.

Results The specificity of most aggrecan-specific T-cell hybridomas mapped to the aggrecan $G_{1}$ domain using synthetic peptides representing known $\mathrm{H}-2^{\mathrm{d}}$ restricted or DR1-restricted CD4 T-cell epitopes. However, most cloned (and recloned) T-cell hybridomas recognised two of the $\mathrm{H}$-2 $2^{\mathrm{d}}$-restricted epitopes (84-103 and 169-189) as well as two of the DR1-restricted epitopes (148-165 and 292-311). The same synthetic peptides were not recognised by $\mathrm{H}-2^{\mathrm{d}}$ restricted and DR1-restricted T-cell hybridomas of unrelated specificities and were not mitogenic for spleen cells from BALB/c or DR1-tg mice. None of the T-cell hybridomas recognised aggrecan epitopes 68--82 or 201-213.

Conclusion We are investigating this unexpected pattern of cross-reactivity between a number of known arthritogenic epitopes of aggrecan using lymph node T-cell responses of peptide-immunised BALB/C and DR1-tg mice. In addition we are investigating the mechanisms of antigen presentation of these epitopes, which may shed light on why this particular region of aggrecan is antigenic for autoreactive T cells.

\section{References}

1. Glant TT, Mickecz K, Arzoumanian A, Poole AR: Proteoglycan induced arthiritis in BALB/c mice. Clinical features and histopathology. Arthritis Rheum 1987, 30:201-212.

2. Hill JA, Wang D, Jevnikar AM, Cairns E, Bell DA: The relationship between predicted peptide-MHC class II affinity and T-cell activation in a HLADRbeta1*0401 transgenic mouse model. Arthritis Res Ther 2003, 5:R40R48.

P7

\section{The role of hypoxia in rheumatoid tendon disease}

\section{B Sivakumar 1,2, N Kang', P Taylor ${ }^{2}$, EM Paleolog ${ }^{2,3}$}

${ }^{1}$ Restoration of Appearance of Function Trust, Northwood, UK; ${ }^{2}$ Kennedy Institute of Rheumatology, Faculty of Medicine, Imperial College, London, UK; ${ }^{3}$ Division of Surgery, Anaesthetics and Intensive Care, Faculty of Medicine, Imperial College, London, UK

Arthritis Res Ther 2005, 7(Suppl 1):P7 (DOI 10.1186/ar1528)

Background Tendon disease is often the first presentation of rheumatoid arthritis (RA). Tenosynovial proliferation results in scarring and adhesion formation. Synovial invasion into tendons occurs in $50 \%$ of cases and is associated with multiple tendon ruptures and a poorer prognosis. Recent work on diseased joints suggests that hypoxia may play a key role in synovial invasion. We hypothesised that hypoxia promotes and maintains the inflammatory response in RA tenosynovium via neovascularisation.

Objectives To measure oxygen tension $\left(\mathrm{pO}_{2}\right)$ within the synovium of rheumatoid tendons and joints in vivo. To study markers of hypoxia and neovascularisation in invasive and non-invasive tendon synovium by immunohistochemistry. To investigate the effects of hypoxia on in vitro cultures of invasive and non-invasive tenosynovium.

Methods Patients undergoing elective hand surgery for RA were recruited into the study. In vivo oxygen tension measurements were taken intra-operatively using an established microelectrode technique. Readings were taken from tendon and joint synovium. Diseased tissue was harvested from areas of oxygen sampling for immunohistochemical analysis of CD31 expression.

Tissue was also harvested from invasive and non-invasive tenosynovium. Joint tissue was used as a control. Serial sections of tissue were stained for the proangiogenic factor vascular endothelial growth factor (VEGF) and the hypoxiaregulated transcription factor hypoxia inducible factor (HIF)- $2 \alpha$.

Separate tissue from these three areas of interest was enzymatically digested and cultured in hypoxic ( $1 \%$ oxygen) and normoxic ( $21 \%$ oxygen) conditions. Supernatants were harvested at 24 hours and analysed for expression of key inflammatory cytokines by ELISA.

Results We observed profound hypoxia in the synovium of RA tendons and joints in vivo despite immunohistochemical evidence of markedly increased vascularity, measured as expression of CD31. Immunohistochemical analysis revealed twofold greater VEGF expression in tenosynovium $(P<0.05)$, compared with joint synovium from the same patient. Levels of HIF- $2 \alpha$ were found to be similar in tendon and joint synovium.

One of the consequences of synovial hypoxia could be the modulation of inflammatory and angiogenic cytokines. We found that hypoxia upregulated in vitro expression of pro-angiogenic cytokine VEGF in both joint and tenosynovial cultures $(P<0.05)$ by an average of $128 \%$, and in parallel increased levels of the pro-inflammatory cytokine tumour necrosis factor alpha by an average of $105 \%$. In contrast, hypoxia downregulated expression of monocyte chemoattractant protein-1 $(P<0.05)$ by an average of $44 \%$.

Conclusions Hypoxia is a feature of rheumatoid tenosynovitis. Key cytokines are regulated by hypoxia in this disease process. HIF- $2 \alpha$ may represent the link between hypoxia and VEGF-driven angiogenesis in rheumatoid tendon disease. Acknowledgements BS is a Royal College of Surgeons of England Research Fellow. The Kennedy Institute of Rheumatology receives a core grant from arc.

P8

Short-fibre modification facilitates adenovirus-mediated gene delivery in rheumatoid arthritis synoviocytes and synovium: role of RGD and non-RGD binding integrins M-L Toh ${ }^{1}$, S-S Hong'2, L Franqueville², L Lindholm³ ${ }^{3}$ P Boulanger ${ }^{2}$, P Miossec ${ }^{1}$

${ }^{1}$ Department of Immunology and Rheumatology, Mixed Unit Civil Hospital of Lyon-Biomerieux, Edouard Herriot Hospital, Lyon, France; ' Laboratory of Virology and Viral Pathogenesis, CNRS UMR-5537, Faculty of Medicine RTH Laennec, Lyon, France; ${ }^{3}$ Department of Medical Microbiology and Immunology, University of Göteborg, and Got-A-Gene AB, Göteborg, Sweden

Arthritis Res Ther 2005, 7(Suppl 1):P8 (DOI 10.1186/ar1529)

Objective Adenovirus 5 (Ad5) receptors other than Cocksackie-ad receptor have not been well explored in rheumatoid arthritis (RA) human fibroblast-like synoviocytes (FLS). Defining these receptors may enable development of improved Ad5 vectors to specific synovial targets. As Cocksackie-ad receptor is absent in RA FLS, we investigated the efficacy and receptor usage of fibre-modified Ad5 vectors for gene delivery in vitro and ex vivo.

Methods Short fibre modified vectors with seven fibre repeats (Ad5GFP-R7knob, Ad5GFP-R7-RGD) were compared with wild-type (WT) 22 repeats 
(Ad5GFP-FiWT) and GFP expression measured in RA human FLS and a synovium explant model. Integrin expression was determined by flow cytometry. Receptor usage was examined by competition binding assays with viral penton base (PB) WT, RGD and LDV-mutant peptides, and antibodies against PB and integrins. Viral neutralization by synovial fluid was examined.

Results Ad5GFP-R7-knob gene transduction was 25-fold higher than Ad5GFPR7-RGD and 40-fold to 50-fold higher than Ad5GFP-FiWT in FLS. Ad5GFP-R7knob gene transduction was reduced with $P B, R G D$ and LDV, and antibodies against PB and $\alpha v, \alpha v \beta 3, \alpha v \beta 5$, and $\beta 1$ integrins. Ad5GFP-R7-knob transduction was increased by tumour necrosis factor and IL-1, remained 30 -fold to 40 -fold higher than Ad5GFP-FiWT in the presence of synovial fluid and preferentially transduced synovium explant outgrowth/tissue.

Conclusion Ad5GFP-R7-knob has broadened viral tropism superior to RGDmodified and WT vectors, mediated by integrin-binding RGD and non-RGD motifs in viral PB capsomers in RA FLS. Ad5 short fibre modifications utilize tropism of the native PB to optimise integrin usage as both attachment and endocytotic receptors. This may be a useful strategy of enhancing gene delivery in RA, reducing vector dose and induction of inflammation and toxicity.

\section{P9}

\section{Laminin type 1 augments the transforming growth factor beta-induced expression of matrix metalloproteinase 3 in synovial fibroblasts}

\section{Hoberg, M Rudert, WK Aicher}

Department of Orthopedic Surgery, University Medical Center, Tuebingen, Germany Arthritis Res Ther 2005, 7(Suppl 1):P9 (DOI 10.1186/ar1530)

Background and objective Elevated expression of laminins (LN) and integrins in the synovial membrane of rheumatoid arthritis (RA) versus osteoarthritis patients has been reported but metabolic effects of attachment of synovial fibroblasts (SF) to $L N$ are not well studied. We therefore investigated gene expression patterns in SF upon attachment to LN1 (EHS laminin) in comparison with LN10.

Methods Expression of IL-1 $\alpha$, IL-1 $1 \beta$, IL-6, IL-8, IL-16, IL-18 as well as matrix metalloproteinase (MMP)-1 and MMP-3 were investigated in RA SF $(n=6)$ or osteoarthritis $\mathrm{SF}(n=7)$ in primary or early passage cultures. Fibroblasts were seeded onto LN1-coated vessels (BD BioCoat ${ }^{\circledR}$ ) for 24-72 hours and cells attached to cell culture vessels served as controls in all experiments. In addition, cells were activated with cytokines and growth factors such as transforming growth factor beta (TGF- $\beta$ ). After activation, transcript amounts of individual genes were enumerated by quantitative RT-PCR. A recombinant cytokine standard and GAPDH RT-PCR served as controls in each sample. Expression of the $\alpha 1, \beta 1$ and $\gamma 1$ chains of LN1 laminin by SF was investigated by quantitative RTPCR and immunocytochemistry. The secretion of MMPs was enumerated by ELISA in SF supernatants.

Results Growth of SF on LN1-coated surfaces without additional stimuli induced a significant IL-8 mRNA response (3.1-fold, $\pm 1.24, P \leq 0.001)$ and lower responses for IL-1 $\beta$, IL-16, IL-1 $\alpha$, IL-18 and IL-6. MMP-1 mRNA was upregulated 2.3-fold ( $\pm 0.79, P \leq 0.001)$, and MMP-3 mRNA only 1.5-fold. Upon incubated of SF on LN10, cytokine and MMP expressions were not changed. Addition of TGF- $\beta$ $(10 \mathrm{ng} / \mathrm{ml}, 24$ hours) to SF attached to tissue culture vessels showed a different induction profile. Here IL- $6, \mathrm{IL}-1 \beta, \mathrm{IL}-1 \alpha, \mathrm{IL}-8, \mathrm{IL}-16$ and MMP-1 mRNAs were induced to some degree, IL-18 mRNA was reduced whereas MMP-3 mRNA was induced (3.15-fold, $\pm 0.7, P \leq 0.04$ ), when compared with controls. Next combinations of activation by TGF- $\beta$ and laminin signaling were investigated. For cytokine expressions no additive effects of combining these signals were seen and MMP-1 mRNA was induced to some extent (threefold, \pm 1.76 ). In contrast, MMP-3 mRNA was induced more that 10 -fold $(12.8 \pm 8.7, P \leq 0.025)$ and MMP-3 secretion was elevated almost 20 -fold $(19.67 \pm 6.9, P \leq 0.06)$. In SF, mRNAs encoding $\alpha 1, \beta 1$ and $\gamma 1$ laminin - which encode the proteins for $\mathrm{LN} 1$ - were detected by quantitative RT-PCR and transcript amounts encoding the $\alpha 1$ and $\beta 1$ chains of LN1 were higher than mRNA encoding the LN1 $\gamma 1$ chain. Using an anti-EHS serum, LN1 was detected on SF by immunocytochemistry. However, using monoclonal antibodies to laminin $\alpha 1$ or $\gamma 1$ proteins, staining signals were very weak.

Conclusions Attachment to LN1-laminin in the presence of TGF- $\beta$ induces elevated MMP-3 expression in SF. However, an autocrine stimulation of MMP-3 expression by SF via TGF- $\beta$ and LN1 seems rather unlikely, as LN1 is not expressed in high amounts in the adult synovial membrane. Still, activation of SF by LN1 may serve as a model for activation of fibroblasts by extracellular matrix compounds in the presence of growth factors or cytokines, and both pathways contribute to the aggressive invasive growth of $\mathrm{SF}$ in the course of RA.
P10

Peptide mimetics of anti-dsDNA idiotypes as a tool for lupus-specific IVIG preparation: specificity and efficacy in the treatment of experimental systemic lupus erythematosus

M Blank'1, I Nur'2, R Meidler², L Bar², L Slutzki', Y Shoenfeld ${ }^{1}$

${ }^{1}$ The Center for Autoimmune Diseases, Department of Medicine 'B', Sheba Medical Center, Tel-Hashomer, Israel; ${ }^{2} \mathrm{OMRIX}$ Biopharmaceuticls Inc., Nes-Ziona, Israel Arthritis Res Ther 2005, 7(Suppl 1):P10 (DOI 10.1186/ar1531)

Background Since the idiotypic network is an important mechanism for controlling the immune repertoire, we tested anti-idiotypic modulation employing concentrated specific natural polyclonal anti-dsDNA anti-idiotypic antibodies obtained from a commercial IVIG in the treatment of experimental systemic lupus erythematosus (SLE).

Aim To address the specificity and efficacy of affinity purified IVIG, affinity purified on peptide mimetics of anti-dsDNA idiotypes, in vitro and in vivo, as treatment for experimental lupus.

Materials and methods Specific natural polyclonal anti-dsDNA anti-idiotypic antibodies were affinity purified from IVIG (OMRIX Biopharmaceuticls Inc., NesZiona, Israel) on an anti-dsDNA-Sepharose column constructed with anti-dsDNA idiotypes affinity purified from 55 patients with SLE. This compound improved the clinical manifestations of NZBXWXF1 mice in 200 times lower concentration than IVIG. This lupus-specific IVIG was introduced to a peptide phage display library (C-7mer-C). The identified synthetic peptides (idiotype mimetics) were synthesized and used to replace the human anti-dsDNA idiotypes column. IVIG affinity purified on the synthetic peptides columns were determined as peptidespecific IVIG (psIVIG). The psIVIG compound was tested for specificity by ELISA and competition assays.

Results Each psIVIG inhibited the binding of anti-dsDNA antibodies from 12 lupus patients, to dsDNA, differentially by $15 \%$ up to $46 \%$ or as a mix up to $87-94 \%$. Naîve mice immunized with a branched peptide composed of the synthetic mimetics of anti-dsDNA idiotypes induced the generation of elevated titers of anti-dsDNA. The anti-dsDNA generation was inhibited in the branced peptide immunized mice, following treatment with psIVIG. A cocktail of psIVIG was introduced to NZBxWxF1. The following clinical parameters were improved in the NZBxWxF1 psIVIG subjected mice: circulating anti-dsDNA antibodies, leukopenia, proteinuria and immunoglobulin deposits in the kidneys.

Conclusion We introduce herein an IVIG subfraction, specific for anti-dsDNA treatment for lupus patients, and discuss its efficacy and beneficial effect in suppression of humoral and clinical signs of SLE versus regular IVIG.

\section{P11}

Acceleration of apoptotic cells clearance by macrophages upon exposure to serum amyloid $\mathbf{P}$ component and its synthetic derivates

\section{Slutzky', M Blank', G Goddard', M Fridkin², Y Shoenfeld'}

${ }^{1}$ Department of Medicine $B$ and The Center for Autoimmune Diseases, Sheba Medical Center, Tel-Hashomer, Israel; ${ }^{2}$ Department of Organic Chemistry, The Weizmann Institute of Science, Rehovot, Israel

Arthritis Res Ther 2005, 7(Suppl 1):P11 (DOI 10.1186/ar1532)

Background Impaired clearance of apoptotic cellular debris by macrophages was recently suggested as a mechanism leading to the systemic pathogenesis of systemic lupus erythematosus patients. Serum Amyloid P component (SAP) is a plasma protein that binds apoptotic cells and macrophages.

Objective To investigate the ability of SAP protein and its synthetic peptide derivates to enhance the clearance of apoptotic cells by macrophages in vitro. Methods Macrophages were isolated from peripheral blood cells of 30 healthy donors and the purity was confirmed by FACS analysis using CD14-PE. Apoptosis was induced in Jurkat cells using anti-Fas antibodies and confirmed by staining with a caspACE ${ }^{\mathrm{TM}}$ FITC-VAD-FMK. The apoptotic cells were introduced to macrophages in the presence/absence of SAP protein and SAP synthetic peptide derivatives. Clearance of labeled apoptotic cells by macrophages, in vitro, was detected by FACS and analyzed under fluorescence microscope. Results In the presence of SAP, the clearance of apoptotic cells increased twice (index 2.03) compared with the control (index 1). In the presence of SAP derivate synthetic peptide (marked as peptide 26), an increase of $53 \%$ (index 1.53) was detected. A D-form of the SAP synthetic derivate peptide was used as a negative control and gave a clearance index of only 1.1 in comparison with the control. A significant correlation was found between the concentration of SAP protein and SAP synthetic peptide derivates and the clearance index.

Conclusion Herein we show the role of SAP and SAP-derived synthetic peptides in accelerating clearance of apoptotic cells by macrophages in vitro. Our results may pave the way for future development of SAP-derived synthetic peptides for apoptotic cell clearance improvement in systemic lupus erythematosus patients. 


\section{P12}

Close association between valvular heart disease and central nervous system manifestations in antiphospholipid syndrome I Krause ${ }^{1,2}$, S Lev ${ }^{2}$, A Fraser ${ }^{2}$, M Blank'1, J Chapman ${ }^{3}$, Y Shoenfeld ${ }^{1,4}$

${ }^{1}$ Research Center for Autoimmune Diseases, Sheba Medical Center, TelHashomer, Israel; ' 2 Department of Medicine 'E', Rabin Medical Center, Beilinson Campus, Sackler Faculty of Medicine, Tel-Aviv University, Israel; ${ }^{3}$ Department of Neurology, Sheba Medical Center, Tel-Hashomer, Israel; ${ }^{4}$ Department of

Medicine 'B', Sheba Medical Center, Tel-Hashomer, Israel

Arthritis Res Ther 2005, 7(Suppl 1):P12 (DOI 10.1186/ar1533)

Objectives Heart valve lesions and central nervous system (CNS) involvement are among the most common manifestations of antiphospholipid syndrome (APS). We evaluated possible inter-relations between these manifestations in a large group of APS patients.

Methods Two hundred and eighty-four APS patients were retrospectively evaluated, 159 of whom had primary APS (PAPS). Cardiac-CNS associations were determined for the entire study population, and for subgroups of patients with PAPS or APS secondary to systemic lupus erythematosus (SLE).

Results Significant associations where found between cardiac vegetations and epilepsy $(P<0.02)$, and between cardiac valves thickening or dysfunction and migraine $(P=0.002)$. Borderline association was found between valvular vegetations and migraine $(P=0.09)$. Subanalyses revealed that patients with PAPS had significant associations between cardiac valve pathology and both epilepsy and migraine, while patients with APS secondary to SLE presented no such associations. Conclusions Our study points to potentially different biological behaviors in PAPS than in APS secondary to SLE. According to our results, the presence of cardiac valves pathology may be a risk factor for several CNS involvements in PAPS.

\section{P13}

\section{Elevated titers of anti-ribosomal-P antibodies in systemic lupus erythematosus}

G Zandman-Goddard', B Gilburd', S Bardechevski', L Stojanovich', P Langevitz ${ }^{2}$, Y Shoenfeld ${ }^{1}$

'Center for Autoimmune Diseases and Department of Medicine ' $B$ ', Sheba Medical Center, Tel-Hashomer, Israel; 2Rheumatology Unit, Sackler Faculty of Medicine, Tel-Aviv University, Israel

Arthritis Res Ther 2005, 7(Suppl 1):P13 (DOI 10.1186/ar1534)

Objective Ribosomal $\mathrm{P}$ is located in the cytoplasm. The detection of antibodies is to the 60 kda fraction. Anti-ribosomal P antibodies are highly specific for systemic lupus erythematosus (SLE), and are detected at a 15-20\% frequency according to the literature. Elevated anti-ribosomal $\mathrm{P}$ titers correlate with disease activity and are specifically associated with neuropsychiatric disease such as psychosis/depression. and coexist with anti-dsDNA antibodies. The aim of our study was to evaluate the frequency of anti-ribosomal P antibody titers and the correlation with manifestations in SLE patients.

Methods Sera samples from 174 individuals were evaluated for titers of antiribosomal P antibodies: 77 samples from SLE patients, 22 patients with antiphospholipid syndrome (APS), 20 patients with familial Mediterranean fever, 12 patients with infections, and 43 healthy controls. Anti-ribosomal P antibody titers were tested by ELISA. Manifestations of SLE at the time of serum sampling were determined by the SLEDAl score.

Results Six SLE patients (11\%) harbored elevated anti-ribosomal P antibody titers. Five SLE patients were females, mean age 44.3 years (range, 18-73 years old), and the mean SLEDAI mean score was 7 (range, 3-10) indicating moderate disease. Elevated titers of anti-dsDNA were detected in 50\% of SLE patients with elevated anti-ribosomal P antibodies. One patient had secondary APS. One patient with elevated titers of anti-ribosomal had renal disease and psychosis. Three patients had a rash, while none of the patients had arthritis or leukopenia. Anti-ribosomal $\mathrm{P}$ titers were not elevated in patients with primary APS, familial Mediterranean fever, infections, or in healthy controls.

Conclusion The prevalence of elevated titers of anti-ribosomal P antibodies was restricted to SLE patients. No correlation with a specific manifestation was found.

\section{P14}

\section{Human type II collagen is processed in lysosomal compartments of macrophages for presentation of the glycosylated arthritogenic epitope $\mathrm{hCll}_{259-273}$ to $\mathrm{CD} 4 \mathrm{~T}$ cells in HLA-DR1 transgenic mice}

A von Delwig', DM Altmann'2, JD Isaacs', R Holmdahl' ${ }^{3}$, N McKie', JH Robinson ${ }^{1}$ ${ }^{1}$ Musculoskeletal Research Group, University of Newcastle upon Tyne, UK; ${ }^{2}$ Human Disease Immunogenetics Group, Imperial College School of Medicine, London, UK; ${ }^{3}$ Section for Medical Inflammation Research, Lund University, Lund, Sweden Arthritis Res Ther 2005, 7(Suppl 1):P14 (DOI 10.1186/ar1535)

Background Post-translational modification of human type II collagen (hCll) in the form of hydroxylation of Pro and Lys residues and glycosylation of some hydroxylated Lys residues has been shown to correlate with $\mathrm{hCll}$ arthritogenicity in susceptible strains of mice [1,2]. At the epitope level, O-linked glycosylation of Lys $_{264}$ located within the arthritogenic region $\mathrm{hCll}_{259-273}$ has been implicated in the creation of neoepitopes recognized by arthritogenic T cells [3]. Macrophages and to lesser extent primed B cells have been implicated in processing hCll for presentation of $\mathrm{hCll}_{259-273}$ epitope to specific T cells $[4,5]$, whereas Langerhans dendritic cells are unable to process CII [6]. Macrophages may thus play a pivotal role in activation of autoreactive $\mathrm{T}$ cells during collagen-induced arthritis. However, no information is available on the mechanisms of antigen processing of the glycosylated arthritogenic epitope, although it is likely to be crucial for an understanding of the activation of autoimmune T cells in rheumatoid arthritis.

Objective We investigated the mechanisms of intracellular processing of $\mathrm{hCll}$ for presentation of the glycosylated epitope $\mathrm{hCll}_{259-273}$ to $\mathrm{CD} 4 \mathrm{~T}$ cells in macrophages from HLA-DR1-transgenic mice.

Methods HLA-DRB ${ }^{\star} 0101$ C57BL/6JO-O transgenic mice (designated HLA-DR1 tg) were developed by backcrossing HLA-DRB $01011^{+} \mathrm{Aq}^{+}$mice onto a $\mathrm{MHC}$ class II-deficient background. T-cell hybridomas specific for the glycosylated and non-glycosylated $\mathrm{hCll}_{259-273}$ epitope were developed to study antigen presentation of the glycosylated epitope by bone marrow macrophages used as antigenpresenting cells. Subcellular fractions of macrophages were used as a source of enzyme activity to digest $\mathrm{hCll}$ at $\mathrm{pH} 4.5$ in the presence and absence of enzyme inhibitors to localize stages of $\mathrm{hCll}$ degradation to particular endosomal/lysosomal compartments and to identify the families of enzymes involved.

Results HLA-DR1-tg mice lacking mouse MHC class II were susceptible to collagen-induced arthritis. Macrophages from DR1-tg mice processed intact hCll for presentation of the glycosylated epitope $\mathrm{hCll}_{259-274}$ to $\mathrm{T}$-cell hybridomas. T-cell hybridomas specific for the glycosylated peptide did not cross-react with the nonglycosylated peptide. Intracellular processing of $\mathrm{hCll}$ for presentation of the glycosylated epitope was prevented by inhibitors of serine-proteases, cysteine-proteases, aspartic-proteases and metallo-proteinases or agents that raise endosomal $\mathrm{pH}$, suggesting a requirement for extensive lysosomal processing. Lysosome-enriched subcellular fractions of macrophages were identified as the main organelles involved in processing and presentation of the glycosylated epitope from hCll, as these compartments contained: proteolytic enzymes of the serine-proteinase and cysteine-proteinase families that could generate the glycosylated hCll epitope; the glycosylated $\mathrm{hCll}$ epitope itself generated by intracellular processing of $\mathrm{hCll}$; peptide-receptive HLA-DR1 molecules; and complexes of HLA-DR1 molecules with the glycosylated and non-glycosylated $\mathrm{hCll}_{259-274}$ epitopes.

Conclusion We showed stringent conditions for intracellular lysosomal processing of $\mathrm{hCll}$ for presentation of the arthritogenic glycosylated epitope by HLA-DR1 molecules to CD4 T cells, which may explain the lack of tolerance to glycosylated collagen and induction of arthritis in HLA-DR1-tg mice.

References

1. Bäcklund J, Treschow A, Bockermann R, Holm B, Holm L, IssazadehNavikas S, Kihlberg J, Holmdahl R: Glycosylation of type II collagen is of major importance for $\mathrm{T}$ cell tolerance and pathology in collageninduced arthritis. Eur J Immunol 2002, 32:3776-3784.

2. Myers LK, Myllyharju J, Nokelainen M, Brand DD, Cremer MA, Stuart JM, Bodo M, Kivirikko KI, Kang AH: Relevance of posttranslational modifications for the arthritogenicity of type II collagen. J Immunol 2004, 172:2970-2975.

3. Bäcklund J, Carlsen S, Hoger T, Holm B, Fugger L, Kihlberg J, Burkhardt H, Holmdahl R: Predominant selection of T cells specific for the glycosylated collagen type II epitope (263-270) in humanized transgenic mice and in rheumatoid arthritis. Proc Natl Acad Sci USA 2002, 99:99609965.

4. Manoury-Schwartz B, Chiocchia G, Fournier C: Processing and presentation of type II collagen, a fibrillar autoantigen, by $\mathrm{H}-2^{q}$ antigen-presenting cells. Eur J Immunol 1995, 25:3235-3242.

5. Holmdahl M, Vestberg M, Holmdahl R: Primed B cells present type-II collagen to T cells. Scand J Immuno/ 2002, 55:382-389.

6. Holmdahl M, Grubb A, Holmdahl R: Cysteine proteases in Langerhans cells limits presentation of cartilage derived type II collagen for autoreactive T cells. Int Immunol 2004, 16:71 7-726.

Acknowledgements Supported by grant MP/R0619 from the Arthritis Research Campaign UK.

\section{P15}

Does the burden of inflammation determined prospectively in patients with early inflammatory arthritis determine the progression of atherosclerosis?

WY Al-Shehhi', S Skehan², E Hefferenan'2, F Hughes², DJ Veale', B Bresinihan ${ }^{1}$, O Fitzgerald ${ }^{1}$

${ }^{1}$ Department of Rheumatology, UCD, Dublin, Ireland; 2 Department of Radiology, UCD, Dublin, Ireland

Arthritis Res Ther 2005, 7(Suppl 1):P15 (DOI 10.1186/ar1536)

Background Accelerated atherosclerosis accounts for the increased mortality seen in patients with established rheumatoid arthritis (RA). Recent research has 
shown that RA is associated with an increase in the carotid intimal medial thickness (IMT). To date, there is no prospective study in RA that examines this question and none that includes an inflammatory disease control.

Objectives To evaluate prospectively the burden of inflammation in a cohort of patients with early inflammatory arthritis, including RA and psoriatic arthritis (PsA). To correlate inflammatory burden with IMT in this cohort and to compare with normal age-matched control subjects.

Methods RA and PsA patients with disease duration of more than 5 years were selected from the early arthritis clinic database for further study. Controls without inflammatory conditions were recruited from the general clinic. Men older than 40 years and women older than 50 years were excluded. Patients with known predisposing risks for ischaemic heart disease were also excluded. At the time of clinical assessment blood samples were collected for erythrocyte sedimentation rate (ESR) and C-reactive protein (CRP) together with fasting lipid samples, and carotid artery ultrasound was performed at the same time. The mean IMT was then calculated by averaging the values at three points, $1 \mathrm{~cm}$ above, at, and $1 \mathrm{~cm}$ below the area of maximal IMT for each carotid artery.

Results To date, 61 patients (29 RA, 26 PsA and six control subjects) have been reviewed. Twenty-one (72\%) of the RA patients compared with $11(42 \%)$ of the PsA patients had an increased IMT (normal IMT $<0.5 \mathrm{~cm}$ ). Mean \pm standard deviation IMT values in the RA cohort $(0.6 \pm 0.14)$ and in the PsA cohort $(0.5 \pm 0.15)$ were significantly greater than controls $(P=0.003, P=0.03$, respectively). IMT values in the RA cohort were greater than in PsA $(P=0.017)$. There was a significant correlation between IMT and age in the RA cohort. However, mean age in the three groups was not different and no correlation between IMT and age was seen in either the PsA or the control group. The area under the curve (AUC) ESR correlated with the AUC CRP in the group as a whole and in both RA and PsA separately. No correlation was found between the AUC for ESR or CRP and IMT in the different subgroups or in patients with an increased IMT.

Conclusions Patients with inflammatory arthritis (RA > PsA) are at a greater risk of developing atherosclerosis compared with control subjects. No correlation has been found to date between IMT values and the burden of inflammation as measured by ESR and CRP over time. Some additional mechanism of accelerated atherosclerosis in inflammation, yet to be determined, may well apply. The results are preliminary and recruitment with additional analysis is ongoing.

\section{P16}

\section{Altered composition of CD97 splice variants in rheumatoid} synovial tissue

FA van Gaalen', TCTM van der Pouw Kraan², RGHH Nelissen³, TWJ Huizinga ${ }^{1}$, CL Verweij $^{2}$

${ }^{1}$ Department of Rheumatology, Leiden University Medical Center, Leiden, The Netherlands; 'Department of Molecular Cell Biology, Free University Medical Center, Amsterdam, The Netherlands; ${ }^{3}$ Department of Orthopedic Surgery, Leiden University Medical Center, Leiden, The Netherlands Arthritis Res Ther 2005, 7(Suppl 1):P16 (DOI 10.1186/ar1537)

The intimal lining of synovial tissue consists of fibroblast-like synoviocytes (type A synoviocytes) and macrophages (type B synoviocytes). These cells are able to interact through the CD97/CD55 receptor ligand system. The CD97 receptor is a heterodimer receptor composed of an alpha and a beta subunit. The CD97alpha subunit may exist as three isoforms due to alternative spliced transcripts, each having a different affinity for CD55.

When comparing synovium of rheumatoid arthritis (RA) patients with synovium of patients with osteoarthritis (OA), no increase in the level of total of CD97alpha or in the level of CD97beta transcripts was found. However, in RA synovium a relative increased expression of CD97 transcripts encoding isoforms with an increased affinity for CD55 (CD97alpha2 and CD97alpha3 mRNA) was detected. A difference in CD97 splice variants between RA and OA tissues could not be attributed to differences in composition of cells as in both tissues the majority of $\mathrm{CD} 7^{+}$cells were CD14+ macrophages/monocytes - 93\% in RA and $94 \%$ in $\mathrm{OA}$, respectively.

These data indicate that during inflammation CD97 splicing is regulated, and we propose that this is likely to affect trafficking and functioning of CD97-positive leukocytes.

\section{P17}

Improvement of T-cell function in rheumatoid arthritis (RA) patients in clinical remission is associated with the recovery of IL-7 expression and depends on a familial history of RA F Ponchel, AK Brown, SL Field, L Hensor, P Emery, JD Isaacs

Molecular Medicine Unit and Rheumatology Research Unit, The University of Leeds, UK

Arthritis Res Ther 2005, 7(Suppl 1):P17 (DOI 10.1186/ar1538)

Background Rheumatoid arthritis (RA) is a chronic, inflammatory disease with a number of phenotypic and functional T-cell defects. We previously demonstrated with inflammation and associated with reduced thymic activity as measured by T-cell recepter excision circle (TREC) content. However, it is still not clear whether these dysfunctions are a primary feature of RA or only secondary to inflammation. Therefore we analysed RA patients in clinical remission in whom systemic inflammation was controlled.

Methods Patients were defined as 'in remission' when they had controlled disease, with no change of activity for at least 6 months, C-reactive protein below 15 , no swollen or tender joints and on stable treatment (with or without therapy). Measurements of TREC content in $\mathrm{CD}^{+}{ }^{+} \mathrm{T}$ cells was by real-time PCR. IL-7 quantification was by ELISA. Proliferation assay in response to mitogen, IL-2, TCR stimulation or recall antigen.

Results The TREC content of CD4 ${ }^{+} \mathrm{T}$ cells in these patients was heterogeneous $(n=35)$. However, two groups of patients could be defined: group 1 (TREC rich), similar to healthy controls ( $n=17$ ); group 2 (TREC poor), close to active disease $(n=19)$. No conventional clinical parameter such as age, sex, disease duration, remission duration, treatment, clinical scores or rheumatoid factor could explain this difference. However, age at disease onset was significantly distributed between these two groups $(P=0.021)$ and correlated with a familial maternal history of RA. IL-7 is essential to thymic T-cell development and to T-cell activation in the periphery. We found large variations in IL-7 circulating levels between RA patients in remission $(n=57)$. There was a positive correlation between the circulating levels of IL-7 and the TREC content $(r=0.701$, $P<0.0001$ ), suggesting that recovery of thymic activity was limited by the availability of IL-7. We investigated T-cell responses to mitogen, antigen, recall antigen and IL-2 in patients with high and low levels of circulating IL-7 ( $n=5$ for each group). Low levels of IL-7 were associated with hyporesponsiveness to all stimuli, which could be overcome by adding IL-7 to the cultures. In contrast, high levels of IL-7 were associated with optimal responses to all stimuli including recall antigen and were not greatly ameliorated by supplementing IL-7.

Conclusion RA patients in clinical remission are clearly separated in two groups, according to their ability to express IL-7 and the associated consequences on thymic activity and T-cell responses. This ability was dependent on the age of the patients at disease onset. This was only associated with a maternal family history of RA and suggests that genetic anticipation may be involved.

Reference

1. Ponchel F, Morgan A, Bingham S, Quinn M, Buch M, Verburg R, Henwood A, Douglas S, Masurel A, Conaghan $P$, et al:: Dysregulation lymphocyte proliferation and differentiation in patients with rheumatoid arthritis. Blood 2002, 100:4550-4556.

P18

T-bet expression in rheumatoid arthritis patients with early, disease-modifying anti-rheumatic drug naïve disease is low and correlates with low levels of IL-7 and T-cell dysfunctions

F Ponchel, AK Brown, SL Field, M Quinn, P Conaghan, P Emery, JD Isaacs

Molecular Medicine Unit and Rheumatology Research Unit, The University of Leeds, UK

Arthritis Res Ther 2005, 7(Suppl 1):P18 (DOI 10.1186/ar1539)

Background Rheumatoid arthritis (RA) exact pathogenesis remains uncertain, although autoimmune processes appear to play a role, and RA is often referred to as a Th1-driven disease. However, it is well known that RA patients have a reduced capacity to produce IL-2 and interferon gamma (IFN- $\gamma$ ). IL-7 is an important cytokine during early events leading naïve $\mathrm{T}$ cells towards Th1 polarisation. We have associated low levels of circulating IL-7 with reduced thymyc T-cell development and T-cell functions in the periphery. The origin of IL-7 in the circulation is not clear and stromal cells in the bone marrow, liver, gut and lymph nodes are potential production sources. The only cytokines known to regulate the expression of IL-7 in different tissues are IFN- $\gamma$ (bone marrow), transforming growth factor beta (TGF- $\beta$ ) (skin) and both IL-1 and tumour necrosis factor alpha (TNF- $\alpha$ ) (synovium). We therefore investigated the relationship between levels of circulating IL-7, a Th1 (IFN- $\gamma$ ) and a Th2 (TGF- $\beta$ ) cytokine, a proinflammatory cytokine (TNF- $\alpha$ ) and two transcription factors essential for T-cell polarisation (T-bet and GATA3).

Methods Patients 'in remission' were defined as having controlled disease, with no change of activity for at least 6 months, C-reactive protein below 15, no swollen or tender joints and on stable treatment (with or without therapy). A cohort of these patients was selected on the basis of their high and low levels of circulating IL-7. RA patients with active, early, disease-modifying anti-rheumatic drug (DMARD) naive disease were also recruited. Circulating levels of cytokines were measured by ELISA and the expression of T-bet and GATA3 by real-time PCR on mRNA extracted from peripheral blood mononuclear cells.

Results We selected 10 patients with either low or high levels of circulating IL-7. Levels of IFN- $\gamma$, TGF- $\beta$ and TNF- $\alpha$ were measured. High levels of IL- 7 were significantly associated with higher levels of IFN- $\gamma(P=0.04)$ and a tendency for lower levels of TGF- $\beta$ but no difference was found for TNF- $\alpha$. Furthermore, there was a 
direct correlation between levels of IL-7 and IFN- $\gamma(R=0.650, P=0.005)$. It is established that a high ratio of expression between T-bet and GATA3 defined a tendency towards Th1 polarisation. T-bet levels of expression were significantly higher in patients with high IL-7 $(P<0.0001)$ and we found a positive correlation between the two $(R=0.600, P=0.011)$. Similar results and correlation were found using the ratio of T-bet to GATA3 expression $(P<0.0001$ and $R=0.700$, $P=0.002$ ). These results were confirmed in a cohort of 10 patients with active, early, DMARD naïve disease.

Conclusion These results confirm that Th1 polarisation is impaired in RA. In addition, they suggest that either the Th1 cytokines regulate the levels of circulating IL-7 or that the transcription factors regulating Th1 polarisation also regulate the expression of IL-7. Further studies on the mode of regulation for IL-7 expression in the circulation are necessary to understand how this cytokine may participate to the pathogenesis of RA.

P19

\section{The signalling signature downstream of the notch receptor in} CD4+CD25 high regulatory $T$ cells in RA defines anergic cells: insight into resistance to anti-tumour necrosis factor therapies MH Buch ${ }^{1}$, K Snow ${ }^{2}$, S Field ${ }^{2}$, P Emery', J Isaacs ${ }^{3}$, F Ponchel ${ }^{2}$

${ }^{1}$ Academic Unit of Musculoskeletal Disease, University of Leeds, UK; ${ }^{2}$ Molecular Medicine Unit, University of Leeds, UK; ${ }^{3}$ Department of Rheumatology, University of Newcastle, UK

Arthritis Res Ther 2005, 7(Suppl 1):P19 (DOI 10.1186/ar1540)

Background We have previously reported on the abnormal expression of the different members of the Jagged/Notch signalling pathway in early rheumatoid arthritis (RA) patients. HES1 and Deltex are two major signalling molecules resulting from the transduction of notch signals. We have shown that signalling through the Jagged/Notch pathway is involved in the development of an anergic phenotype in a T-cell clone model in vitro. We have also used this model to establish a Notch signalling signature characterising a T-cell suppression reaction. We analysed the Notch signature of effecter $\mathrm{CD} 4^{+} \mathrm{T}$ cells and $\mathrm{CD} 4^{+} \mathrm{CD} 25^{\text {high }}$ regulatory $\mathrm{T}$ cells in RA patients and compared it with healthy controls.

Methods The HA1.7 CD4+T-cell clone develops an anergic phenotype associated with high $C D 25$ expression when stimulated with its cognate peptide in the absence of co-stimulation. These cells were used in a suppression assay of fresh HA1.7 (CD25low) cells, activated by anti-CD3/CD28 antibodies in proliferation assays. Real-time PCR for the quantification of gene expression was undertaken. $\mathrm{CD} 4^{+} \mathrm{CD} 25^{-} \mathrm{T}$ cells and $\mathrm{CD} 4{ }^{+} \mathrm{CD} 25^{\text {high }}$ regulatory $\mathrm{T}$ cells were sorted using flow cytometry from $50 \mathrm{ml}$ blood from five RA patients with early, disease-modifying anti-rheumatic drug naive disease and seven healthy controls. Gene expression was quantified by real-time PCR.

Results During a suppression reaction of fresh HA1.7 cells (CD25 low) by anergic HA1.7 expressing high levels of CD25, the expression of HES1 was induced; however, Deltex was inhibited. This signature is unique and differs from either an activation (downregulation of both) or an anergy signalling signature (upregulation of both). Results from a similar quantification in RA patients, suggest that CD4 ${ }^{+} \mathrm{CD} 25_{\text {high }} \mathrm{T}$ cells in RA have the signature of anergic cells with high levels of both HES1 and Deltex, whereas control regulatory cells display a signature comparable with previously published data (low HES1, high Deltex) [1]. Preliminary results in RA patients, pre and post TNF-blockade, suggest that the response to therapy is associated with a normalisation of the Notch signature post treatment to resemble the signature of control activated regulatory $\mathrm{T}$ cells (upregulated HES1 and downregulated Deltex).

Conclusions $\mathrm{CD} 4{ }^{+} \mathrm{CD} 25$ high regulatory $\mathrm{T}$ cells in RA appear anergic and may therefore not be able to suppress an undesirable auto-immune reaction very early in the RA disease process. Altogether, our results on the abnormal baseline expression of Jagged/Notch molecules and these atypical signalling signatures suggest that the Jagged/Notch pathway may be involved in the pathogenesis of RA. Reference

1. Ng WF, Duggan PJ, Ponchel F, Matarese G, Lombardi G, Edwards AD, Isaacs JD, Lechler Rl: Human CD4(+)CD25(+) cells: a naturally occurring population of regulatory T cells. Blood 2001, 98:2736-2744.

P20

Citrullinated $\alpha$ enolase, a novel citrullinated autoantigen in rheumatoid arthritis, upregulated by chronic inflammation A Kinloch, V Tatzer, R Wait, D Peston, S Sacre, P Donatien, D Moyes, $P$ Taylor, PJ Venables

Inflammation \& Immunity, Kennedy Institute of Rheumatology, Imperial College London, UK

Arthritis Res Ther 2005, 7(Suppl 1):P20 (DOI 10.1186/ar1541)

Introduction Antibodies to citrullinated proteins are the most specific serological marker for rheumatoid arthritis (RA). They are associated with severity of disease and may occur years before clinical manifestations. It is unclear whether antibodies to citrullinated proteins react with any citrullinated protein or whether there are
Figure 1

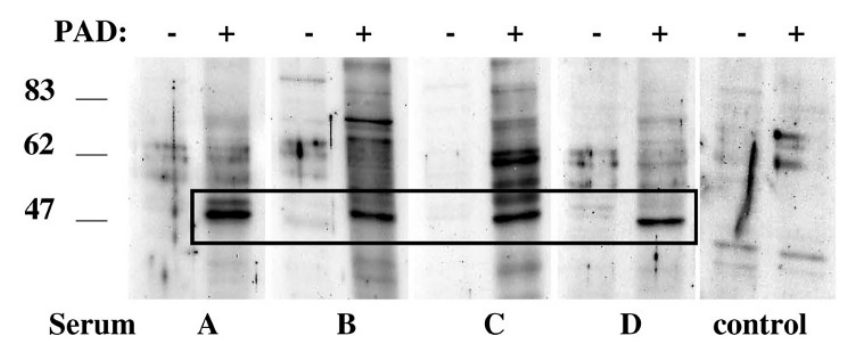

Reactivity of rheumatoid arthritis and healthy serum with non-citrullinated/ citrullinated lysates of HL60 monocytes. PAD, peptidyl arginine deiminase.

specific citrullinated proteins driving the autoimmune response. In this study we use the HL60 cell line as a substrate for identifying autoantigens in myelomonocytic cells, the major cell type present in the rheumatoid joint.

Method Citrullinated autoantigens were sought by immunoblotting with a screening panel of four RA and one healthy control sera, using lysates of HL60 cells, untreated or differentiated to monocytes or granulocytes. Each lysate was incubated with or without peptidyl arginine deiminase in vitro. Reactive bands were identified using two-dimensional gel electropheresis and MALDI TOF microsequencing. IgG antibodies to purified $\alpha$-enolase, incubated with or without peptidyl arginine deiminase, were detected by immunoblotting with 53 RA and 40 healthy control serum samples. Immunohistochemistry of synovial sections and immunoblotting of lysed synoviocytes was performed using anti- $\alpha$-enolase antibody diluted to 1:400. Macrophages stimulated with lipopolysaccharide or tumour necrosis factor were derived from primary monocytes, differentiated with monocyte colony-stimulating factor.

Results By immunoblotting, a band of $47 \mathrm{kDa}$ in the deiminated lysates of HL60s differentiated to monocytes and granulocytes reacted selectively with the four RA sera (Fig. 1, sera A-D), but not with the control serum. It was identified as citrullinated $\alpha$-enolase by microsequencing and confirmed by immunoblotting of twodimensional subcellular fractions with an anti- $\alpha$-enolase antibody and RA sera. In a larger panel of sera, $24(46 \%)$ of the RA samples reacted with citrullinated $\alpha$ enolase, of which seven also reacted with the uncitrullinated form. Six (15\%) normal sera reacted with both forms. $\alpha$-Enolase was demonstrated in the subsynovium and vascular endothelial cells in all synovial membrane biopsies. This was confirmed by immunoblotting where a protein in RA synoviocyte lysates, co-migrating with purified $\alpha$-enolase, reacted with anti- $\alpha$-enolase antibody. Stimulation of macrophages with lipopolysaccharide or tumour necrosis factor upregulated and induced secretion of $\alpha$ enolase. Secretion was inhibited with monensin.

Conclusions In this study, nearly one-half of the RA sera reacted with citrullinated $\alpha$-enolase. This is a high frequency given the relative insensitivity of immunoblotting. We suggest that using a more refined ELISA-based assay, both diagnostic sensitivity and specificity will increase. The finding of antibody, together with abundant expression of antigen in the joint (which is amplified with inflammatory stimuli) makes citrullinated $\alpha$-enolase a candidate autoantigen for driving the chronic immune response in RA.

Acknowledgment This work was supported by the Arthritis Research Campaign (arc).

P21

Abatacept (CTLA4-Ig) modulates human T-cell proliferation and cytokine production but does not affect lipopolysaccharide-induced tumor necrosis factor alpha production by monocytes

PM Davis, SG Nadler, KA Rouleau, SJ Suchard

Bristol-Myers Squibb Pharmaceutical Research Institute, Princeton, New Jersey, USA Arthritis Res Ther 2005, 7(Suppl 1):P21 (DOI 10.1186/ar1542)

Background and objectives Activated T cells play a central role in the inflammatory cascade leading to the joint inflammation and destruction characteristic of rheumatoid arthritis (RA). The cytokines secreted by activated T cells are thought to both initiate and propagate the immunologically driven inflammation associated with RA.

Abatacept, the first of a new class of agents for the treatment of RA that selectively modulates the co-stimulatory signal required for full T-cell activation, was evaluated for its ability to regulate human T-cell proliferation and cytokine production in vitro. The effect of abatacept on lipopolysaccharide (LPS)-induced tumor necrosis factor alpha (TNF- $\alpha$ ) from monocytes was evaluated to distinguish the impact of this agent on innate versus adaptive, antigen-specific immune responses.

Methods T cells were isolated from normal healthy human volunteers. The effect of abatacept on antigen-dependent T-cell activation was evaluated using either an irradiated human B-cell line (PM-LCL) as the antigen-presenting cells (APCs) for a primary mixed lymphocyte reaction (MLR), or autologous E-PBMCs as APCs 
for a recall response to tetanus toxin (TT). Cytokines were measured at various times post activation, with proliferation determined on day 5 . Monocytes were isolated by elutriation, challenged with LPS and TNF- $\alpha$ levels measured at 6 hours. Chi L6 was included as a non-specific fusion protein control.

Results Abatacept significantly downmodulated T-cell proliferation, in both primary and recall responses, at concentrations between 0.3 and $100 \mu \mathrm{g} / \mathrm{ml}$, with maximal inhibition ( 60-80\%) observed at $\sim 3-10 \mu \mathrm{g} / \mathrm{ml}$. These concentrations are below the abatacept trough plasma levels observed in patients receiving a clinically effective dose [1]. Under conditions of maximal inhibition of proliferation, and similar to trough plasma levels in patients $(30 \mu \mathrm{g} / \mathrm{ml})$, abatacept also inhibited IL-2, TNF- $\alpha$ and interferon gamma secretion in both primary and TT-dependent recall responses. However, the extent, kinetics and rank order of cytokine inhibition by abatacept was somewhat different between primary and recall responses. In contrast, abatacept did not inhibit LPS-induced TNF- $\alpha$ production in primary human monocytes, demonstrating that its action is restricted to antigen-dependent T-cell responses.

Conclusion Abatacept, a selective co-stimulation modulator, significantly inhibited the activation (as measured by cytokine production) and proliferation of human $\mathrm{T}$ cells in the context of a primary MLR or TT-dependent memory response. This inhibition occurred at concentrations below the serum $\mathrm{C}_{\text {min }}$ levels observed in patients receiving a clinically effective dose of abatacept [1] $(10 \mathrm{mg} / \mathrm{kg}$ monthly), consistent with suppression of T-cell activation in vivo. There was no effect of abatacept on LPS-stimulated TNF- $\alpha$ production in monocytes indicating that this agent may largely preserve innate immune responses.

\section{Reference}

1. Kremer JM, Westhovens R, Leon M, Di Giorgio E, Alten R, Steinfeld S, Russell A, Dougados M, Emery P, Nuamah IF, et al.: Treatment of rheumatoid arthritis by selective inhibition of T-cell activation with fusion protein CTLA4Ig. N Engl J Med 2003, 349:1907-1915.

\section{P22}

Intra-articular gene therapy blocking NF-אB using adenoassociated virus type 5 ameliorates adjuvant arthritis in rats J Adriaansen', SW Tas', N Hajji', A Bakker'2, MJ Vervoordeldonk', PP Tak'

${ }^{1}$ Academic Medical Center, Amsterdam, The Netherlands; ${ }^{2}$ Amsterdam

Molecular Therapeutics, Amsterdam, The Netherlands

\section{Arthritis Res Ther 2005, 7(Suppl 1):P22 (DOI 10.1186/ar1543)}

Objective NF- $\mathrm{KB}$ is highly activated in synovium of RA patients, and can induce transcription of proinflammatory cytokines, adhesion molecules, and inducible nitric oxide, among others. Phosphorylation of the inhibitor of $\kappa \mathrm{B}(\mathrm{I} \mathrm{KB})$ proteins is an important step in NF-KB/Rel activation and is regulated by IKB kinase (IKK). The IKK complex consists of at least three subunits, including IKK $\alpha$ and IKK $\beta$ (also called IKK1 and IKK2) and the regulatory subunit IKK $\gamma$. In an initial study in Lewis rats with adjuvant arthritis (AA) adenoviral dominant-negative IKK2 (Ad.IKK2dn) significantly ameliorated the severity of disease as evidenced by decreased paw swelling compared with Ad.GFP-treated rats [1]. However, adenoviral vectors are known to be very immunogenic, compromising stable long-term expression of the transgene. Adeno-associated virus (AAV) is considered the most promising vector for gene therapy in RA. In a comparative serotype study we found that direct injection of AAV5 into the ankle joints of rats with AA resulted in the highest synovial transduction, with good expression of the transgene at the protein level until the end of the study, followed by AAV2. In the present study we investigated the effect of inhibiting NF-KB in AA in rats using AAV-mediated intra-articular gene therapy. For this purpose we used the following vectors: AAV5 containing the IKK2dn gene (AAV5.IKK2dn) or AAV2 containing the IKB $\alpha$-supressor gene (AAV2.IKB $\alpha \mathrm{SR}$ ). Methods AAV5.IKK2 dn $\left(2.5 \times 10^{10} \mathrm{vp}\right)$, AAV2.IKB $\alpha$ SR $\left(2.5 \times 10^{10} \mathrm{vp}\right)$ or AAV5/ AAV2.GFP were injected into the right ankle joints of rats with AA on day 11 after adjuvant immunization. Subsequently, the effect of both genes on paw swelling was measured by water displacement plethysmometry. Animals were sacrificed 2 weeks after intra-articular injection and joints were collected for analysis. Bone degradation was examined using $\mathrm{X}$-rays of the ankle joints and histology was performed to assess synovial inflammation and joint damage.

Results In the rats treated with IKK2dn, significantly reduced paw swelling was observed ( $P<0.05$, AAV5.dnIKK2 versus AAV5.GFP). No significant effect was found on cartilage and bone destruction. Intra-articular treatment of the rats with $A A V 2 . I K B \alpha S R$ only showed a marginal effect on the clinical course of arthritis. Conclusion We demonstrate that AAV5-mediated IKK2dn gene transfer to the synovium reduces the severity of inflammation in AA rats, when the treatment was started after the onset of disease. In contrast, injection of AAV2.IKB $\alpha$ SR had a poor clinical effect on paw swelling in rats with AA. This could either be due to the dissimilarities in the mechanisms that these genes use to inhibit NF-KB activation (IKK2 versus $1 \mathrm{~K} B \alpha$ ) or to the use of different $A A V$ serotypes as vector (AAV5 versus $A A V 2$ ). Reference

1. Tak PP, Gerlag DM, Aupperle KR, Aupperle KR, van de Geest DA, Overbeek $\mathrm{M}$, et al:: Inhibition of nuclear factor kappa $\mathrm{B}$ kinase beta is a key regulator of synovial inflammation. Arthritis Rheum 2001, 44:1897-1907.
P23

Collagenous repeat containing sequence of $\mathbf{2 6} \mathrm{kDa}$ protein: a novel mesenchymal gene of cartilage and adipose tissue differentiation

IH Tarner', C Büchler1, J Schölmerich', E Neumann'1, S Gay², A Schäffler ${ }^{1}$, U Müller-Ladner ${ }^{1}$

${ }^{1}$ Department of Internal Medicine I, University Hospital Regensburg, Germany; ${ }^{2}$ Center for Experimental Rheumatology, Department of Rheumatology, University of Zurich, Switzerland

Arthritis Res Ther 2005, 7(Suppl 1):P23 (DOI 10.1186/ar1544)

Background Collagenous repeat containing sequence of $26 \mathrm{kDa}$ protein (CORS-26) is a recently discovered novel member of the TNF/C1q/adiponectin superfamily. CORS-26 has been detected in chondrocytes and osteoblasts, but its functional properties are largely unknown. As CORS-26 shows structural analogies to adiponectin, which has been shown to exert proinflammatory and destructive properties in arthritic synovium, the goal of the present study was to examine the expression and regulation of CORS-26 in adipocyte differentiation. Methods Gene expression of 3T3-L1-preadipocyte cultures were examined on the protein and mRNA level by real-time PCR, Western blot, electrophoretic mobility shift assay and luciferase-reporter gene assay.

Results CORS-26 showed numerous homologies to adiponectin such as a Cterminal globular domain and a N-terminal collagen domain. CORS-26 mRNA expression could not be detected in preadipocytes and in early adipocytes after 48 hours of culture. After 4-9 days of adipocyte differentiation, however, upregulation of CORS-26 mRNA and protein could be detected. CORS- 26 promoter activity and RNA expression could be stimulated by troglitazone and fenofibrate but not by 15-deoxy-prostaglandin J2. In addition, PPAR $\gamma$, but not PPAR $\alpha$, binds specifically to a promoter response element at position $-641 /-596$.

Conclusions CORS-26 appears to be a novel adiponectin with strong homologies to the other members of the C1q/TNF/adiponectin superfamily, which includes its functional properties that may be as proinflammatory in the arthritic joint as those of adiponectin.

\section{P24}

\section{Activation of antigen-presenting cells by endogenous retroviral RNA}

D Moyes', S Sacre', N Temperton'2, A Lundberg', B Foxwell', P Venables'

${ }^{1}$ The Kennedy Institute, Imperial College London, UK; ${ }^{2}$ Wohl Virion Centre,

University College London, UK

Arthritis Res Ther 2005, 7(Suppl 1):P24 (DOI 10.1186/ar1545)

Background Several studies have linked human endogenous retroviruses (HERVs) with autoimmune disease. We have previously demonstrated that the full-length polymorphic HERV-K113 provirus prevalence is increased in both $\mathrm{Sjö}^{-}$ gren's syndrome $(15.6 \%)$ and multiple sclerosis $(11.9 \%)$ compared with the normal UK population (4\%). Recent studies have shown that exogenous viral RNA can activate dendritic cells via the TLR7/8-mediated pathway. In this study we investigate the possibility that HERV-K113 RNA sequences are capable of activating both dendritic cells and macrophages. We examine the production of cytokines by both cell types in response to a series of different HERV-K113 RNA species to identify any differences in the profile produced.

Methods A clone of the full-length HERV-K113 provirus was digested with restriction enzymes to generate different length fragments of the $5^{\prime}$-LTR and gag gene for in vitro transcription, producing single-stranded RNA species. The RNA was treated to remove any residual protein, DNA, lipopolysaccharide or other contaminants. After quantitation, the RNA was complexed with Lipofectamine 2000 (Invitrogen, Paisley, UK) or left uncomplexed before being used to treat cells. Cytokines levels in the cell supernatants were measured by ELISA. Dendritic cells were assayed for tumour necrosis factor (TNF), IL-6 and IP-10, while macrophages were assayed for TNF, IL-6, IL-8 and IL-10. Controls used were un-complexed RNA, polyU synthetic RNA, and the chemical activator of TLR 7 and 8, Resiquimod (R-848).

Results Stimulation with R-848 produced TNF, IL-6, IL-8 and IL-10 from macrophages and TNF, IL-6 and IP-10 from dendritic cells (Table 1). Uncomplexed RNA did not stimulate cytokine production in any cell type demonstrating a lack of lipopolysacharide or other contaminant activation of cells. Stimulation of dendritic cells with each of the three complexed RNA species resulted in the production of IL-6, TNF and IP-10 (Table 1). Stimulation of macrophages only produced TNF. Although low levels of IL-6 were detected, there was no increase over control cells (Table 1). Neither IL-8 nor IL-10 was produced in macrophages in response to any of the RNA species. All three RNA species were more active than a poly-U synthetic RNA control.

Conclusions The data demonstrate that RNA from HERVs is capable of inducing cytokine release by both macrophages and dendritic cells. The pattern of cytokine expression differs between the two cell types and may reflect different activation pathways in each case. This work demonstrates a possible mechanism of action for HERV induction of an inflammatory response leading to an autoimmune disease. 
Table 1

Cytokine release in response to one species of HERV-K113 RNA from different cells

\begin{tabular}{lccc}
\hline & $\begin{array}{c}\text { Tumor } \\
\text { necrosis } \\
\text { factor } \\
(\mathrm{ng} / \mathrm{ml})\end{array}$ & $\begin{array}{c}\mathrm{IL}-6 \\
(\mathrm{ng} / \mathrm{ml})\end{array}$ & $\begin{array}{c}\mathrm{IP}-10 \\
(\mathrm{ng} / \mathrm{ml})\end{array}$ \\
\hline Macrophage and K113 RNA & 5.46 & 0.234 & Not done \\
Macrophage and R848 (positive control) & 5.78 & 15.56 & Not done \\
Macrophage and control RNA (negative control) & 0.04 & 0.0 & Not done \\
Dendritic cell and K113 RNA & 4.45 & 3.75 & 5.2 \\
Dendritic cell and R848 (positive control) & 6.49 & 13.57 & 5.12 \\
Dendritic cell and control RNA (negative control) & 0.095 & 0.458 & 0.824 \\
\hline
\end{tabular}

\section{P25}

Anti-tumour necrosis factor alpha treatment in patients affected by rheumatoid arthritis with anti-Ro/SSA antibodies I Cavazzana, F Franceschini, E Danieli, P Airò, R Cattaneo

Rheumatology and Clinical Immunology Unit and Chair, Spedali Civili, Brescia, Italy Arthritis Res Ther 2005, 7(Suppl 1):P25 (DOI 10.1186/ar1546)

Background Anti-tumour necrosis factor alpha (anti-TNF- $\alpha$ ) treatment could induce the onset of new autoantibodies $[1,2]$ or clinical features of other autoimmune diseases than rheumatoid arthritis (RA) [2].

Aim To analyse the efficacy and safety of anti-TNF- $\alpha$ treatment in six patients affected by RA with anti-Ro/SSA antibodies and to evaluate the clinical and laboratory changes during the treatment.

Patients and methods Antinuclear antibodies (IFI on HEp-2 cells), anti-dsDNA (Farr assay), anticardiolipin (aCL, ELISA) and anti-ENA (CIE) were evaluated before the treatment and every 6-12 months. Anti-Ro/SSA antibodies were confirmed by ELISA, using recombinant 52 and $60 \mathrm{kDa}$ Ro proteins (Pharmacia).

Results Six patients had anti-Ro/SSA antibodies before anti-TNF $\alpha$ treatment. Anti-Ro/SSA was detected in four sera using both CIE and ELISA, while two sera showed anti-60 kDa Ro antibodies only by ELISA. Six patients (mean age, 58 years; standard deviation [SD], 9.8) were affected by long-acting RA (mean duration, 7 years; range, 5-22 years), not responding to common disease-modifying anti-rheumatic drugs (mean, 5 disease-modifying anti-rheumatic drugs; SD, 2.3). All the patients were treated for a mean of 31 months (SD, 20.4 months), four subjects with Infliximab and two with Etanercept. Before the treatment the patients showed active arthritis with a mean of 25.3 tender joints (SD, 2.16), 17.3 swollen joints (SD, 8.6) and Disease Activity Score (DAS) of 5.5 (SD, 1.04); after $6,12,18$, and 24 months all the patients showed a rapid and sustained improvement with reduction of DAS value (see Table 1).

All the patients were affected by Sjögren syndrome (SS), associated to RA, clinically stable during the treatment. One subject was also affected by primary biliary cirrhosis, which remained clinically and histologically stable. Three patients developed antidsDNA at low titre after 6 months and a fourth after 12 months of treatment. Only one patient developed skin lesions after 6 months of Infliximab, clinically and histologically similar to subacute cutaneous lupus with lgM deposits at basal membrane. No patients developed aCL, while the titre of anti-Ro antibodies by ELISA was stable during the treatment. One subject, affected by RA and HCV hepatitis, stopped the etanercept treatment due to severe increase of hepatic enzymes.

\section{Table 1}

Clinical response to anti-tumour necrosis factor alpha treatment in patients with rheumatoid arthritis and anti-Ro/SSA antibodies

\begin{tabular}{lcc}
\hline & Mean Disease Activity Score 44 & $P$ \\
\hline At onset & 5.56 & \\
6th month & 3.38 & 0.006 \\
12th month & 3.41 & 0.03 \\
18th month & 3.33 & 0.01 \\
24th month & 2.88 & 0.006 \\
\hline
\end{tabular}

Conclusions Anti-TNF treatment in RA-SS patients with anti-Ro/SSA showed a good and sustained efficacy until the 24th month. Four patients (66.7\%) showed anti-dsDNA after 6-12 months, while only one developed subacute cutaneous lupus-like symptoms. No other autoantibodies nor an increase of the anti-Ro titre were observed.

References

1. Charles PJ, Smeenk RJ, DeJong J, Feldmann M, Maini RN: Assessment of autoantibodies to double-stranded DNA induced in rheumatoid arthritis patients following treatment with infliximab, a monoclonal antibody to tumor necrosis factor alpha: findings in open-label and randomized placebo-controlled trials. Arthritis Rheum 2000, 43:2383-2390.

2. Cush JJ: Unusual toxicities with TNF inhibition: heart failure and druginduced lupus. Clin Exp Rheumatol 2004, 22(Suppl 35):S141-S147.

\section{P26}

Ha-Ras/ERK1-2 pathway is responsible for oxygen species generation in scleroderma fibroblasts

S Svegliati', MM Luchetti', P Paroncini', A DiCarlo', F Bevilacqua', N Campelli', E Avvedimento ${ }^{2}$, A Gabrielli ${ }^{1}$

${ }^{1} /$ stituto di Clinica Medica Generale, Ematologia ed Immunologia Clinica, Università Politecnica delle Marche, Ancona, Italy; ${ }^{2}$ Dipartimento di Biologia e Patologia cellulare e Molecolare, Università Federico II, Napoli, Italy Arthritis Res Ther 2005, 7(Suppl 1):P26 (DOI 10.1186/ar1547)

Background Reactive oxygen species (ROS) are involved in scleroderma (SSc) fibroblast activation, proliferation and type I collagen gene expression [1]. In order to understand the signal trasduction pathway involved in ROS generation by SSc fibroblasts, we have assessed the role of Ha-Ras and Ki-Ras proteins and that of their downstream effectors.

Materials and methods Intracellular ROS were evaluated with 2',7'-dichlorofluorescein diacetate (DCHF-DA $10 \mu \mathrm{M})$. For Ha-Ras and Ki-Ras protein expression whole cell lysates were immunoprecipitated with specific antibodies and subjected to SDS-PAGE or cells were fixed in paraformaldehyde (4\%) and observed by confocal microscopy. SSc skin fibroblasts were transiently transfected with the dominant negative form of Ha-Ras (H17Ras) or with the empty vector using the Effectene Trasfection reagents (BioRad).

Results Quiescent SSc fibroblasts contain an altered ratio between the two Ras isoforms showing a selective increase of the mass and the activity of Ha-Ras protein when compared with normal controls. Moreover ERK1-2 are constitutively phosphorilated in quiescent SSc fibroblasts. Inhibition of either Ras, ERK1-2, ROS with specific inhibitor or trasfecting with the negative Ras variant reverse this phenotype back to normal and reduce collagen overproduction by SSc cells. Conclusions These results suggest that ROS production in SSc fibroblasts is linked and ultimately dependent on increased Ha-Ras signalling via ERK1-2. These events are responsible for the characteristic cellular phenotype of scleroderma fibroblasts and explain some of the clinical features of the disease. Moreover, these data provide evidence for the use of Ras inhibitors in the treatment of the disease.

Reference

1. Sambo P, Baroni SS, Luchetti M, Paroncini P, Dusi S, Orlandini G, Gabrielli A: Oxidative stress in scleroderma: maintenance of scleroderma fibroblast phenotype by the constitutive up-regulation of reactive oxygen species generation through the NADPH oxidase complex pathway. Arthritis Rheum 2001, 44:2653-2664.

\section{P27}

Magnetic resonance imaging examinations of the spine in patients with ankylosing spondylitis before and after therapy with the tumor necrosis factor alpha receptor fusion protein etanercept

X Baraliakos', J Davis², W Tsuji ${ }^{3}$, J Braun 1

${ }^{1}$ Rheumazentrum Ruhrgebiet, Herne, Germany; ${ }^{2}$ Division of Rheumatology, University of California, San Francisco, California, USA; ${ }^{3}$ Amgen Inc., Thousand Oaks, California, USA

Arthritis Res Ther 2005, 7(Suppl 1):P27 (DOI 10.1186/ar1548)

Objective To assess spinal inflammation by magnetic resonance imaging (MRI) before and after treatment with the tumor necrosis factor (TNF) receptor fusion protein etanercept compared with placebo.

Methods MRI examinations of the lower thoracic and lumbar spine of 40 patients with active ankylosing spondylitis (AS) were performed as part of the recently published randomized controlled trial at four different timepoints: baseline (BL), after 12 weeks (FU1), 24 weeks (FU2) and 48 weeks (FU3). Nineteen patients received etanercept twice weekly, $2 \times 25 \mathrm{mg}$ subcutaneously, for 1 year and 21 patients received placebo for 6 months (until FU2) before switching to etanercept. The patients' (mean age 39.7 years, 75\% male, 89\% HLA-B27-positive) mean disease duration was 13 years. MRI examinations included T1-weighted sequences before (T1) and after application of gadolinium-diethylenetriaminepentaacetic-acid (T1/Gd-DTPA) and T2-weighted fat-saturated (T2-FS) sequences. MRI examinations were scored by a modified ASspiMRI score using predefined vertebral units as a basis. 
Results After 12 weeks, spinal inflammation (T2-FS) regressed by $54 \%(1.33$ mean scoring points per vertebral units at $\mathrm{BL}$ and 0.61 at $\mathrm{FU} 1, P=0.002)$ in the etanercept group, but worsened by $13 \%$ in the placebo group $(0.94$ at $\mathrm{BL}$ and 1.06 at TP1, respectively; $P<0.05)$. After switching to etanercept, placebo patients improved similarly. T1/Gd-DTPA MRI sequences performed equally well. About $60 \%$ of all active lesions at BL were detected in the thoracic spine. There were no significant changes in the chronicity score.

Conclusions Treatment with etanercept of patients with active AS results in regression of spinal inflammation as assessed by spinal MRI. Inclusion of the thoracic spine in MRI examinations of AS patients may be of particular importance.

P28

\section{Two-year follow-up results after re-administration of etanercept in active ankylosing spondylitis}

X Baraliakos', J Brandt' 1 , J Listing' ${ }^{2}$, H Haibel $^{3}$, HM Rudwaleit ${ }^{3}$, J Sieper ${ }^{3}$, J Braun ${ }^{1,3}$

${ }^{1}$ Rheumazentrum Ruhrgebiet, Herne, Germany; ${ }^{2}$ German Rheumatism Research Center, Epidemiology Department, Berlin, Germany; ${ }^{3}$ Department of

Gastroenterology/Rheumatology, Charité, Medical University Berlin, Campus Benjamin Franklin, Germany

Arthritis Res Ther 2005, 7(Suppl 1):P28 (DOI 10.1186/ar1549)

Objectives The tumor necrosis factor alpha (TNF- $\alpha$ ) receptor fusion protein etanercept has proven short-term efficacy in patients with active ankylosing spondylitis (AS). The results of continuous treatment with etanercept over 1 year were reported previously. Here we report the experience with the second year of follow-up.

Methods Overall, 23 out of 30 AS patients (77\%) who had participated in the initial placebo-controlled phase of the trial were included in this 2-year extension, where patients with active disease were treated with etanercept $(2 \times 25$ mg subcutaneously twice a week). Disease-modifying anti-rheumatic drugs and steroids were not allowed. The clinical response was assessed by standard assessment tools for disease activity (Bath AS Disease Activity Index [BASDAI]), function (Bath AS Functional Index) and mobility (Bath AS Metrology Index). The primary outcome of this trial was efficacy on disease activity after 2 years of continuous treatment with etanercept in AS patients, compared with patient status at baseline (BL).

Results Of the 30 initial patients, 21 (70\%) completed year 2. At week 102,54\% of the patients had maintained a 50\% improvement of BASDAl, and 9/21 (43\%) were in a state of partial remission according to the ASAS criteria. The mean BASDAl score remained stable $(2.6 \pm 2.2$ at week 54 and $2.7 \pm 2.4$ at week 102) in the second study year. Similarly, all other clinical parameters showed no change during year 2 with significant improvement compared with BL. Two patients experienced serious adverse events leading to discontinuation of therapy.

Conclusions This study confirms the efficacy and safety of etanercept in the therapy of patients with active AS without simultaneous administration of diseasemodifying anti-rheumatic drugs or steroids over 2 years of continuous treatment.

\section{P29}

\section{Clinical response to the anti-tumor necrosis factor alpha antibody infliximab in patients with ankylosing spondylitis over 3 years}

X Baraliakos', J Brandt', J Listing', J Sieper ${ }^{3}$, J Braun'

${ }^{1}$ Rheumatology Medical Center Ruhrgebiet, Herne, Germany; ${ }^{2}$ German

Rheumatism Research Center, Berlin, Germany; ${ }^{3}$ University Medicine Berlin, Campus Benjamin Franklin, Germany

Arthritis Res Ther 2005, 7(Suppl 1):P29 (DOI 10.1186/ar1550)

Objective Infliximab, a monoclonal antibody against tumor necrosis factor alpha (TNF- $\alpha$ ), is approved in Europe for the treatment of patients with active ankylosing spondylitis (AS) who have responded inadequately to conventional therapy. This report provides analyses from a 3-year extension study, as a follow-up to both the 1-year and 2-year open label extensions of the original 3-month randomized controlled trial of infliximab in patients with AS.

Methods Of the 49 patients with AS who completed the second year of the study, 46 continued treatment with infliximab $5 \mathrm{mg} / \mathrm{kg}$ every 6 weeks through week 156. The Bath AS Disease Activity Index (BASDAI), the Bath AS Functional Index, the Bath AS Metrology Index, patient's and physician's global assessment, quality of life (Short Form-36), C-reactive protein, and erythrocyte sedimentation rate were assessed throughout the study period.

Results The improvement of signs and symptoms observed in the majority of the patients during the first and second year was sustained throughout the third year of the study. Forty-three patients (62\% of the 69 patients enrolled at baseline and $93 \%$ of the patients who started the third year) completed week 156. A BASDAI $50 \%$ improvement was achieved by 28 of 43 patients $(65 \%)$ at week 156 . The scores for other efficacy assessments were similar to the values observed at weeks 54 and 102. Median C-reactive protein levels remained low $(1.5 \mathrm{mg} / \mathrm{l}$ at week 156). There were no relevant side effects and no discontinuation because
Conclusions Patients with AS receiving infliximab for 3 years showed durable clinical response without loss of efficacy. Long-term infliximab treatment was well tolerated by patients in this study.

\section{P30}

Clinical response to discontinuation of anti-tumor necrosis factor therapy in patients with ankylosing spondylitis after 3 years of continuous treatment with infliximab

\section{Baraliakos ${ }^{1}$, J Listing' ${ }^{2}$, J Brandt', M Rudwaleit ${ }^{3}$, J Sieper ${ }^{3}$, J Braun ${ }^{1}$}

${ }^{1}$ Rheumazentrum Ruhrgebiet, Herne, Germany; ${ }^{2}$ German Rheumatism Research Center, Berlin, Germany; ${ }^{3}$ Charité, Medical University of Berlin, Campus Benjamin Franklin, Department of Rheumatology, Germany

Arthritis Res Ther 2005, 7(Suppl 1):P30 (DOI 10.1186/ar1551)

Objective To analyze the clinical response and the time to relapse after withdrawal of continuous long-term infliximab therapy in patients with ankylosing spondylitis (AS).

Methods After 3 years of infliximab therapy, all AS patients $(n=42)$ discontinued treatment (TP1) and were visited regularly in order to assess the time to relapse (TP2) for 1 year. Relapse was defined as an increase to a Bath AS Disease Activity Index (BASDAI) value and physician's global assessment $>4$ according to the ASAS recommendations.

Results After 52 weeks, 41 of the 42 patients $(97.6 \%)$ had to be reinfused because of relapse. The mean change of the BASDAl between TP1 and TP2 was $3.6 \pm 1.7$, and for the physician's global assessment was $4.4 \pm 1.8$ (both $P<0.001)$. The mean time to relapse was 17.5 weeks $( \pm 7.9$ weeks, range 7-45 weeks). Ten patients (24\%) showed a relapse within 12 weeks and 38 patients $(90.5 \%)$ within 36 weeks. After 52 weeks, only one patient had remained in ongoing remission without anti-tumor necrosis factor therapy. Patients who were in partial remission and those with normal C-reactive protein levels at baseline had longer mean time to relapse after discontinuation. Retreatment with infliximab was safe and resulted in clinical improvement similar to the state before withdrawal in all patients.

Conclusions Discontinuation of long-term therapy with infliximab led to relapse of disease activity in all but one patient after different time periods. Remission and low $\mathrm{C}$-reactive protein levels at the time of withdrawal were associated with longer flare free periods. Retreatment with infliximab was safe and efficacious.

\section{P31}

Radiographic progression in patients with ankylosing spondylitis after 2 years of treatment with the tumor necrosis factor alpha antibody infliximab

\section{Baraliakos', J Listing'2, M Rudwaleit ${ }^{3}$, J Brandt', J Sieper ${ }^{3}$, J Braun'}

${ }^{1}$ Rheumazentrum Ruhrgebiet, Herne, Germany; ${ }^{2}$ German Rheumatism Research Center, Berlin, Germany; ${ }^{3}$ Rheumatology, Charité, Campus Benjamin Franklin, Berlin, Germany

Arthritis Res Ther 2005, 7(Suppl 1):P31 (DOI 10.1186/ar1552)

Background Anti-tumor necrosis factor therapy is clinically efficacious in patients with active ankylosing spondylitis (AS) and leads to improvement of spinal inflammation, as assessed by magnetic resonance imaging. It is unclear whether antitumor necrosis factor therapy has influence on chronic spinal changes in AS.

Objective To analyze the effect of infliximab on the radiographic course of AS over 2 years.

Methods Complete sets of lateral radiographs of the cervical and the lumbar spine were available from 82 patients from two sources: 41 patients (group 1) had been treated with infliximab ( $5 \mathrm{mg} / \mathrm{kg} / 6$ weeks) as part of a recent randomized controlled trial, and 41 patients (group 2) were part of the early German AS cohort (GESPIC), without controlled interventions. Radiographs were performed at baseline and after 2 years and were scored by the modified SASSS.

Results Patients in the infliximab group were older, had a longer disease duration and more radiographic damage at baseline. The mean modified SASSS change was $0.4( \pm 2.7)$ and $0.7( \pm 3.4)$ for group 1 and group 2 , respectively $(P=$ not significant). Radiographic damage at baseline was a predictor for more radiographic progression. Patients with baseline damage who were treated with infliximab showed a trend for less radiographic progression. There were no correlations between clinical parameters and radiographic progression.

Conclusions AS patients treated with infliximab showed somewhat less radiographic progression after 2 years. Patients with prevalent radiographic damage are prone to develop more damage over time. Infliximab may decelerate radiographic progression in such patients. Larger studies are needed to prove that anti-tumor necrosis factor therapy inhibits structural damage.

Acknowledgement This abstract is eligible for application for a postgraduate student fellowship. 
P32

Oxygen radical production determines chondrocyte death and regulates matrix metalloproteinase-mediated matrix degradation during interferon gamma-accelerated immune complex arthritis

PLEM van Lent ${ }^{1}$, KCAM Nabbe ${ }^{1}$, AEM Holthuysen ${ }^{1}$, JK Kolls ${ }^{2}$, SM Holland ${ }^{3}$, WB van den Berg1

${ }^{1}$ Department of Rheumatology, UMCN Nijmegen, The Netherlands; ${ }^{2}$ Department of Pediatrics, University of Pittsburgh, Pennsylvania, USA; ${ }^{3}$ Host Defenses, National Institute of Allergy and Infectious Diseases, Bethesda, Maryland, USA

Arthritis Res Ther 2005, 7(Suppl 1):P32 (DOI 10.1186/ar1553)

Objective In earlier studies we found that FcyRl determines chondrocyte death and matrix metalloproteinase (MMP)-mediated cartilage destruction during interferon gamma (IFN- $\gamma$ )-regulated immune complex (IC)-mediated arthritis [1]. As binding of ICs to FcyRl leads to oxygen radical production, we now investigate the contribution of oxygen radicals to induction of both parameters of cartilage destruction using P47phox knockout mice. These mice have a defect in NADPHoxidase activation and fail to produce oxygen radicals.

Methods IFN- $\gamma$ was expressed in knee joints of P47phox ${ }^{-1-}$ and their wildtype (WT) controls by local injection of adenoviral IFN- $\gamma$ construct. One day thereafter, a passive IC-mediated arthritis was induced. Chondrocyte death and MMP-mediated cartilage destruction were measured in various layers of the knee joint using histology of total knee joints. Neoepitopes induced by MMPs were detected using immunolocalisation and anti-VDIPEN antibodies. Synovium was isolated and mRNA levels (MMPs/ tissue inhibitors of metalloproteinases [TIMPs]) were determined using quantitative RT-PCR.

Results High levels of IFN- $\gamma$ were found 1 day after injection of the IFN- $\gamma$ adenoviral construct in knee joints of $\mathrm{P}_{4} 7^{-/-}$and WT controls, and resulted in a high and comparable upregulation of FcyRI up to day 7 in both groups. Induction of ICmediated arthritis in IFN- $\gamma$-treated knee joints resulted in prominent but comparable joint inflammation both at day 3 and day 7 . At day 7, macrophages formed the dominant cell type. When compared with WT controls, RNA levels of MMP-3 and MMP-13, which are crucial in cartilage destruction within this model, were elevated in inflamed synovia of P47phox ${ }^{-/-}$whereas TIMP-3, a crucial inhibitor of MMP-3, was completely absent. Nevertheless, MMP-mediated cartilage destruction was significantly lower in P47 phox $^{-/-}$at day 7 (between 30-60\%). Moreover chondrocyte death that was evident in arthritic WT controls $(20-60 \%$ at day 3 and $30-80 \%$ at day 7) was completely blocked in P47phox ${ }^{-1}$.

Conclusion During IFN- $\gamma$-regulated IC-mediated arthritis, oxygen radicals, which are strongly induced after Fc $\gamma \mathrm{Rl}$ stimulation, determine chondrocyte death and largely mediate MMP-mediated cartilage destruction.

Reference

1. Nabbe KC, van Lent PL, Holthuysen AE, Kolls JK, Verbeek S, van den Berg WB: FcgammaRI up-regulation induced by local adenoviral-mediated interferon-gamma production aggravates chondrocyte death during immune complex-mediated arthritis. Am J Pathol 2003, 163:743-752.

P33

In systemic sclerosis, levels of tissue kallikrein are related to microvascular changes assayed by videocapillaroscopy and immunohistochemistry

A Del Rosso', AF Milia', LI Manneschi' ${ }^{2}$, Distler $^{3}$, S Guiducci', A Pignone', $\mathbf{S}$ Gay $^{3}$, MM Cerinic ${ }^{1}$

${ }^{1}$ Department of Medicine, Division of Rheumatology, University of Florence, Italy; ${ }^{2}$ Department of Anatomy, Histology and Forensic Medicine, University of Florence, Italy; ${ }^{3}$ Center of Experimental Rheumatology, University Hospital of Zurich, Switzerland

Arthritis Res Ther 2005, 7(Suppl 1):P33 (DOI 10.1186/ar1554)

Objective In systemic sclerosis (SSc), characterised by microvascular changes leading to ischemia and impaired angiogenesis, the lack of an angiogenic response to hypoxia may be due to inappropriate synthesis of angiogenic and angiostatic factors. Tissue kallikrein ( $t$-kallikrein) is a potent angiogenic agent regulating the kallikrein kinin system with kallistatin as its natural inhibitor. T-allikrein is synthesised by endothelial and vascular smooth muscle cells as well as inflammatory cells.

Aim To evaluate, in SSc patients: $t$-kallikrein and kallistatin levels and their correlation with clinical features and parameters of microvascular involvement; and $t$-kallikrein expression in the skin of SSc patients.

Methods Serum levels of $t$-kallikrein and kallistatin (ELISA) were assayed in 43 SSc patients, also evaluated for subsets (dSSc, 11 patients; ISSc, 32 patients), clinical and immunological features and microvascular involvement (ulcers, teleangiectasias, nailfold videocapillaroscopy). Expression of t-kallikrein (immunohistochemistry) was evaluated in biopsies taken from the involved skin of the forearm of six SSc patients (two ISSc and four dSSc) and six controls.

Results Circulating levels of $t$-kallikrein were higher in SSc patients than in controls $(n=35)(P<0.001)$. No differences in $\mathrm{t}-$ kallikrein were detected between ISSc and dSSc. T-kallikrein levels were higher in patients with early (five patients) and active (31 patients) capillaroscopic patterns than in those with a late pattern (seven patients) $(P=0.019$ and 0.023$)$. Patients with giant capillaries and capillary microhemorrhages (35 patients) had higher t-kallikrein levels than patients with architectural derangement (8 patients) $(P=0.04)$. Neither differences in kallistatin levels were detected between SSc patients and controls nor between ISSc and dSSc. In control skin samples, t-kallicrein immunoreactivity was shown in endothelial and smooth muscle cells and pericytes of microvessels. In ISSc skin, immunoreaction for t-kallicrein was found in microvascular endothelial cells and in perivascular inflammatory infiltrates present in the papillary dermis near to the dermo-epidermal junction. In dSSc skin, the few remaining vessels showed no immunoreactivity for t-kallicrein, except for the vessels near sweat glands and nerves.

Conclusions Our findings indicate that both t-kallikrein levels and t-kallikrein skin expression are related to typical SSc microvascular changes as assayed by nailfold videocapillaroscopy and by immunohistochemistry of skin biopsies.

\section{P34}

A complete phylogenetic analysis coupling expression data from EST databases. An example with a family of genes: the peptidyl arginine deiminase genes N Balandraud, P Gouret, E Danchin, M Blanc, D Zinn, P Pontarotti

EA 3781 Evolution, Genome, Environment, Université de Provence, Marseille, France Arthritis Res Ther 2005, 7(Suppl 1):P34 (DOI 10.1186/ar1555)

Background For functional annotation, similarity-based approaches [1] do not take into account all the information from comparative and evolutionary biology. They do not differentiate between orthologs and paralogs among homologs and, furthermore, the closest BLAST is often not the nearest neighbour [2]. Phylogenetic approaches taking into account duplication and speciation events are necessary to solve these problems. But they do not blend any data from transcriptional behaviour. Nevertheless, orthologs can have very similar 'molecular function' but undergo a different 'macroscopic function' because of a transcriptional shift.

Growing data for gene expression profiling are available in various databases concerning normal or pathological tissues (Expressed Sequence Tags [ESTs] from NR, TIGR, GeneNote, Gepis, etc.). Some works recently examined the correlation between evolution (duplication and speciation) of genes and expression divergence within and between species $[3,4]$, and some examine the expression profile between orthologous genes in sequenced species [5].

Methods We performed a phylogenetic analysis of a protein family, using EST databases. This allowed us to enlarge the dataset of species containing homologs and consequently to improve the reconstruction of the genes' evolutionary history. We then extracted all the transcriptional data contained in EST databases, to decipher the gene expression pattern. Because gene annotation is currently labour intensive, we used a locally developed platform dedicated to phylogenetic annotation (named FIGENIX) [6]. We validated this approach on a family of genes possibly implied in rheumatoid arthritis; the peptidyl arginine deiminase (PADI) genes.

Results We show here a phylogenetic annotation with an enlarged dataset including EST contigs and expression data. It allowed us to integrate more functional data for analysis of a set of genes and permits us to give a transcriptional footprint of the gene. Our analysis showed that the PADI-2 paralog group have kept the ancestral molecular function coupled with a probable ancestral expression profile. These classified data permitted us to perform an updated footprint of the transcriptional data for each paralog group from this protein family.

Conclusion We believe this method announces a new way to annotate uncharacterized ESTs. More than classical phylogeny, it allows highlighting of the transcriptional shift between paralogs, and is thus a good tool to improve annotation. It showed that functional shift can occur in differential tissue expression rather than in biochemical function of the protein.

This method of analysis is at its beginning and has to be extended to all kinds of expression database, including databases where expression data are normalized such as UniGene. In the future it cannot be ignored in annotating new unknown ESTs, underlined by DNA microarray assays for example.

References

1. Altschul SF, et al.: Gapped BLAST and PSI-BLAST: a new generation of protein database search programs. Nucleic Acids Res 1997, 25:33893402.

2. Koski LB, Golding GB: The closest BLAST hit is often not the nearest neighbor. J Mol Evol 2001, 52:540-542.

3. Gu Z, et al.: Duplicate genes increase gene expression diversity within and between species. Nat Genet 2004, 36:577-579.

4. Huminiecki L, Wolfe KH: Divergence of spatial gene expression profiles following species-specific gene duplications in human and mouse. Genome Res 2004, 14:1870-1879.

5. Yanai I, et al:: Genome-wide midrange transcription profiles reveal expression level relationships in human tissue specification. Bioinformatics 2004, in press [E-pub]. 
6. Gouret P, et al:: 'Intelligent' automation of genomics annotation: expertise integration in a new software platform, Figenix. Genome Res 2004, submitted.

Acknowledgement This work is supported by the French Society of Rheumatology (SFR).

P35

Expression of innate immune receptors in the immunopathologic lesion of primary Sjogren's syndrome: preliminary results

E Bourazopoulou, EK Kapsogeorgou, JG Routsias, DA Liakos, HM Moutsopoulos, AG Tzioufas

Department of Pathophysiology, School of Medicine, National University of Athens, Greece

Arthritis Res Ther 2005, 7(Suppl 1):P35 (DOI 10.1186/ar1556)

Background Toll-like receptors (TLRs) have a crucial role in early host defence against invading pathogens. They recognize specific molecular patterns that are present in microbial and viral components, whereas several endogenous molecules seem to be placed among their expanding group of ligands. The CD91 receptor (low-density lipoprotein receptor-related protein [LRP1] or alpha 2macroglobulin receptor [A2MR]) is a multifunctional receptor that recognizes hsp-chaperoned peptides, which are processed and presented by the MHC class I and MHC class II molecules. So far, there is an increasing evidence that the receptors of innate immunity also play an important role in acquired immunity. Objective The objective of our study was to evaluate the expression of TLRs and CD91 in minor salivary gland (MSG) biopsy tissues and non-neoplastic salivary gland epithelial cell lines obtained from patients with primary Sjogren's syndrome (pSS).

Methods At the mRNA level, the expression of TLR2, TLR4, TLR7, TLR9 and CD91 was examined in a non-neoplastic salivary gland epithelial cell line from a patient with pSS by means of RT-PCR. Protein expression of TLR2, TLR4 and CD91 was studied by immunohistochemistry in MSG biopsy tissues from 12 patients with $\mathrm{pSS}$ and nine control patients with non-specific sialadenitis.

Results RT-PCR analysis revealed transcriptional activation of TLR2, TLR4, TLR7, TLR9 and CD91 genes in the epithelial cell line of pSS patient. In MSG biopsy tissues, abundant expression of CD91 was observed in ductal cells of both pSS and control tissues, but also in the focal lymphocytic infiltrates of pSS patients. TLR2 and TLR4 protein expression was localized in single, scattered cells, both in pSS patients and, to a lesser extent, in control patients, showing a distinct staining pattern.

Conclusion The presence of CD91 and TLRs in the immunopathologic lesion of Sjogren's syndrome provides preliminary evidence, suggesting their possible role in innate immune responses within the lesion.

\section{P36}

\section{The association of different B27 subtypes with the peptide} loading complex

\section{JC Goodall, L Ellis, JSH Gaston}

Department of Medicine, Addenbrookes Hospital, University of Cambridge, UK Arthritis Res Ther 2005, 7(Suppl 1):P36 (DOI 10.1186/ar1557)

Background The molecular basis for the strong association of HLA-B27 with ankylosing spondylitis (AS) has not been elucidated. A number of B27 alleles including $B^{*} 2704$ and $B^{*} 2705$ are associated with increased susceptibility to AS; in contrast, the alleles $B^{\star} 2706$ and $B^{\star} 2709$ are not associated with AS. These alleles differ in amino acid regions that have been shown to influence the interaction with tapasin, an accessory molecule that plays a critical role in incorporating HLA class I into the peptide loading complex (PLC). Our aim was to determine whether B27 subtypes differ in their association with the PLC.

Methods The .221 line that is negative for HLA class I alleles A, B and C was transfected with $B^{\star} 2704,05,06$ and 09 expression constructs, and stable long-term transfectants with equivalent HLA-B27 expression were selected. The PLC was immunoprecipitated using the anti-TAP antibody 148.3 (provided by R Tampe) and magnetic anti-immunoglobulin microbeads. Non-specific binding of HLA class I was determined by immunoprecipitation with an isotype control mAb. Following reducing SDS-PAGE and western blotting of the eluted proteins, the co-precipitation of HLA-B27 was detected by probing the immunoblots with the anti-HLA class I mAb, $\mathrm{HC} 10$, followed by HRP-linked secondary antibodies and visualized using electrochemiluminescence. Detection of TAP and tapasin was achieved by stripping bound $\mathrm{mAbs}$ and reprobing the immunoblots with RING4C (provided by P Cresswell) or 'Giles' (provided by T Elliott) polyclonal antisera, respectively.

Results Only HLA-B*2704, 05 and 09 were found to be readily detectable above background on the immunoblot; suggesting that they were co-precipitated by the anti TAP $\mathrm{mAb}$ and therefore incorporated into the PLC. Despite expressing equivalent quantities of HLA class I heavy chain to the other B27 subypes, HLA$B^{\star} 2706$ was unique in that it was not detectable above background levels. The tation of the PLC since equivalent quantities of TAP and tapasin were immunoprecipitated from the cell lysates of all B27 subtypes.

Conclusions We have shown that $B^{\star} 2706$ differs from the other B27 subtypes in that it cannot be detected within the PLC. B*2706 differs from $B^{\star} 2704$ at amino acid positions 114 and 116 , and we suggest that these amino acid differences confer the PLC independence of this allele. PLC independence may play a role in contributing protection from arthritis by a number of potential mechanisms such as conferring an inability to bind an athritogenic peptide that is only efficiently bound in the context of the PLC. Alternatively, lack of association with the PLC could also modify an event downstream of peptide loading that may protect from disease susceptibility.

\section{P37}

Functional regulatory immune responses against human cartilage glycoprotein-39 in health versus proinflammatory responses in rheumatoid arthritis

JHM van Bilsen'1, H van Dongen', LR Lard', EIH van der Voort', DG Elferink2 ${ }^{2}$ AM Bakker', AMM Miltenburg ${ }^{3}$, TWJ Huizinga', RRP de Vries ${ }^{2}$, REM Toes

${ }^{1}$ Department of Rheumatology, Leiden University Medical Center, Leiden, The Netherlands; ${ }^{2}$ Department of Immunoheamatology and Blood Transfusion, Leiden University Center, Leiden, The Netherlands; ${ }^{3}$ NV Organon, Oss, The Netherlands Arthritis Res Ther 2005, 7(Suppl 1):P37 (DOI 10.1186/ar1558)

The class of immune response against autoantigens could profoundly influence the onset and/or outcome of autoimmune diseases. Until now, there is only limited information on the antigen-specific balance between proinflammatory and regulatory responses in humans. Here we analyzed the natural immune response against a candidate autoantigen in rheumatoid arthritis, Human Cartilage gp-39 (HC gp-39).

Peripheral blood mononuclear cells from healthy individuals reacted against $\mathrm{HC}$ gp-39 with the production of IL-10, but not interferon gamma (Fig. 1). Ex vivo assays indicated that the naturally occurring $\mathrm{HC}$ gp-39-specific immune response in bulk is powerful enough to suppress antigen-specific recall responses, demonstrating that, rather than being unresponsive, the $\mathrm{HC}$ gp-39directed immune response in healthy individuals shows a strong bias towards a regulatory phenotype. Moreover, CD4+ $\mathrm{T}$-cell lines directed against $\mathrm{HC}$ gp-39 expressed CD25, GITR and Foxp3 molecules and were capable of suppressing antigen-specific T-cell responses. Cell-cell contact was required for this suppression. As opposed to healthy individuals, the $\mathrm{HC}$ gp-39-directed immune response in 50\% of patients with rheumatoid arthritis exhibits polarization towards a proinflammatory Th1 phenotype and is significantly less powerful in suppressing antigen-specific recall responses.

Together these findings indicate that the presence of $\mathrm{HC}$ gp-39-specific immune responses in healthy individuals may have an inhibitory effect on inflammatory responses in areas where $\mathrm{HC} \mathrm{gp-39}$ is present. Furthermore, these data indicate that the class of $\mathrm{HC}$ gp-39-directed immune response in RA patients has shifted from an anti-inflammatory towards a proinflammatory phenotype.

Figure 1

(a) HC gp-39 (b) Recall antigens

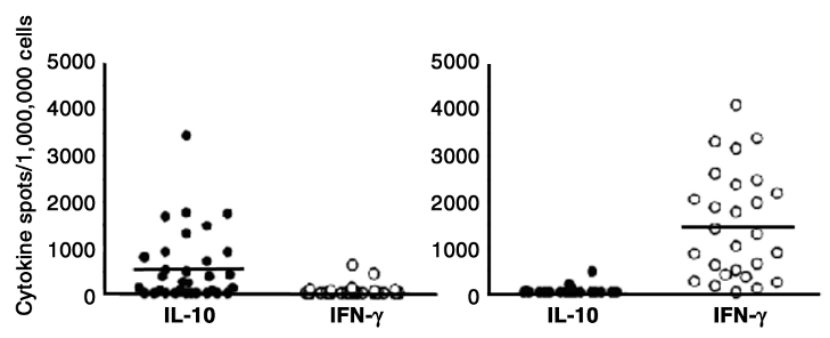

Human Cartilage gp-39 (HC gp-39)-specific immune reactivity in healthy donors. (a) Peripheral blood mononuclear cells from healthy donors $(n=31)$ react against $\mathrm{HC}$ gp-39 by production of IL-10 but not interferon gamma (IFN- $\gamma$ ). (b) 'Control' recall antigen-induced responses are dominated by IFN- $\gamma$ production.

Acknowledgements This work was supported by The Dutch Arthritis Association. REMT is supported by a Vidi Grant from the Netherlands Organization for Scientific Research. HvD and LRL are supported by The Netherlands Organization for Health Research and Development. 
P38

Differential survival of leucocyte subsets by synovial, bone marrow and skin fibroblasts: site-specific rescue of CD4 $T$ cells versus activation-induced rescue of neutrophils AD Filer ${ }^{1}$, G Parsonage 1 , AMCT Thomas ${ }^{2}$, SJ Curnow'1, A Akbar ${ }^{3}$, M Salmon', CD Buckley ${ }^{1}$

${ }^{1}$ Rheumatology Research Group, MRC Centre for Immune Regulation, University of Birmingham, UK; ${ }^{2}$ Royal Orthopaedic Hospital, Birmingham, UK; ${ }^{3}$ Department of Immunology and Molecular Pathology, UCL, London, UK

Arthritis Res Ther 2005, 7(Suppl 1):P38 (DOI 10.1186/ar1559)

Background Chronic inflammatory disease is characterised by predilection for certain sites, the molecular basis of which remains obscure. We investigated the role that fibroblasts play in governing the survival (persistence) as well as the type of infiltrate (predominantly neutrophil versus lymphocyte) using a leucocytefibroblast co-culture model. We used matched synovial, bone marrow and skin fibroblasts to test the hypothesis that a fibroblast-derived, stromal area post code identifies microenvironments that can support differential leucocyte accumulation. Methods Matched synovial (SFb), bone marrow (BMFb) and dermal fibroblast $(\mathrm{DFb})$ lines were established from eight patients satisfying ACR criteria for rheumatoid arthritis undergoing total hip or knee arthroplasty. Activated peripheral blood CD4 T cells prepared by negative depletion and neutrophils prepared by density gradient centrifugation were co-cultured with fibroblasts. Survival, apoptosis and proliferation were assessed using total cell counts and active caspase 3 staining (T cells) or DiOC6 staining (neutrophils).

Results Site-specific survival of CD4 T cells was observed in all eight sets of matched samples: at 3 days, DFb maintained better T-cell survival than culture in medium alone ( $40 \pm 1.2 \%$ (mean \pm standard error) compared with $25.4 \pm 1.4 \%$ $(P<0.05))$. However, both SFb and BMFb maintained even better T-cell survival compared with DFb: $58.8 \pm 1.2 \%(P<0.001$ versus $\mathrm{DFb})$ and $51.4 \pm 2 \%$ $(P<0.05$ versus $\mathrm{DFb})$. Fibroblast pre-activation with a wide range of proinflammatory signals had no effect on differential survival. T-cell survival was partially reconstituted by medium from T cell:fibroblast co-cultures and reduced by $50 \%$ in the presence of transwells suggesting that $T$ cell:fibroblast interactions are necessary for increased survival. Unlike the case for T cells, resting fibroblasts did not affect neutrophil survival. However pre-treatment of all fibroblasts with tumour necrosis factor alpha significantly increased neutrophil survival: SFb $51.2 \pm 6.1 \%$, BMFb $51.1 \pm 5.5 \%$, DFb $50.6 \pm 4.7 \%$, versus control $16.2 \pm 3.3 \%$, $P<0.001$. Fibroblast conditioned media reconstituted the survival effect, indicating that a soluble survival factor(s) as opposed to cell-cell contact mechanism is involved in neutrophil survival.

Conclusions Fibroblasts differentially regulate leucocyte subset survival in both a site-dependent and activation-dependent manner. Our results provide an alternative explanation for site-specific differences in the pattern of T-cell and neutrophil accumulation in different chronic inflammatory diseases.

\section{P39}

\section{Regulatory T-cell defect in the arthritis-susceptible DBA/1 mouse \\ SP Fairchild ${ }^{1}$, M Schenk ${ }^{1}$, R Doffinger ${ }^{2}$, FC Hall ${ }^{1}$}

${ }^{1}$ Department of Clinical Medicine, University of Cambridge, UK; ${ }^{2}$ Department of Clinical Immunology and Biochemistry, Addenbrooke's Hospital, Cambridge, UK Arthritis Res Ther 2005, 7(Suppl 1):P39 (DOI 10.1186/ar1560)

Background DBA/1 mice are susceptible to collagen-induced arthritis and to low-grade spontaneous inflammatory arthritis in elderly males. CD $4^{+} \mathrm{CD} 25^{+}$ T cells have been implicated in the suppression of arthritis in the susceptible DBA/1 strain [1,2] and in patients with rheumatoid arthritis [3]. We have studied the function of the $\mathrm{CD} 4^{+} \mathrm{CD} 25^{+} \mathrm{T}$-cell subset in DBA/1 and arthritis-non-susceptible C57BI/6 male mice.

Methods The frequencies of $\mathrm{CD} 4{ }^{+} \mathrm{CD} 25^{+} \mathrm{T}$ cells were assessed in lymph nodes and the spleen of DBA/1 and C57BI/6 mice by FACS analysis. Spleens were harvested from adult male $\mathrm{DBA} / 1$ and $\mathrm{BALB} / \mathrm{c}$ mice. $\mathrm{CD} 4^{+} \mathrm{CD} 25^{+}$and CD4 ${ }^{+} C D 25^{-} T$ cells were purified using Miltenyi reagents and a VarioMACS. Proliferation assays in response to either Concanavalin A $(1 \mu \mathrm{g} / \mathrm{ml})$ or soluble anti-CD3 $(0.1-10 \mu \mathrm{g} / \mathrm{ml})$ were performed using $5 \mathrm{e} 4 \mathrm{CD} 4^{+} \mathrm{CD} 25^{-}$cells with $1 \mathrm{e} 5$ irradiated adherent splenocytes as antigen-presenting cells. Co-culture of $\mathrm{CD} 4^{+} \mathrm{CD} 25^{+}$and $\mathrm{CD} 4^{+} \mathrm{CD} 25^{-} \mathrm{T}$ cells in a $1: 1$ ratio was used to assess suppression. Cytokines (IL-2, interferon gamma, IL-4, IL-10, tumour necrosis factor alpha, IL-6, IL-17, IL-15) were measured in the tissue culture supernatant after 36 hours incubation. Proliferation was measured by $\left[{ }^{3} \mathrm{H}\right]$-thymidine incorporation. Suppression ratios (SRs) were calculated as follows: $\mathrm{SR}=$ (response from $\mathrm{CD} 25^{+}: \mathrm{CD} 25^{-} \mathrm{T}$-cell coculture) / (response from CD25- T cells alone). SRs in DBA/ 1 and C57BI/6 mice were compared using Student's $t$ test.

Results The frequency of $\mathrm{CD} 4^{+} \mathrm{CD} 25^{+} \mathrm{T}$ cells was comparable in the spleen and lymph nodes of DBA/ 1 and $\mathrm{C} 57 \mathrm{~B} / / 6$ mice. SRs were higher (i.e. less suppression) in proliferation assays from DBA/1 mice compared with C57BI/6 mice, which were stimulated with anti-CD3 $(P=0.03)$. DBA/1 cultures were generally associated with higher cytokine levels. Cytokine SRs were higher (i.e. less suppression) for DBA/ 1 compared with C57B//6 cultures for IL-6 and IL-17. SRs were lower (i.e. more suppression) for DBA/1 compared with C57BI/6 cultures for IL-4 and IL-10. Conclusions The arthritis-susceptible DBA/1 mouse strain appears to have a functional defect in the $\mathrm{CD} 4+\mathrm{CD} 25^{+} \mathrm{T}$-cell compartment. IL-4 and IL-10 are efficiently suppressed by $\mathrm{CD} 4^{+} \mathrm{CD} 25^{+} \mathrm{T}$ cells. Suppression of IL- 6 and IL-17 appears to be defective. This may partly explain the susceptibility of DBA/1 mice to erosive arthritis. References

1. Morgan ME, Sutmuller RP, Witteveen HJ, van Duivenvoorde LM, Zanelli E, Melief CJ, Snijders A, Offringa R, de Vries RR, Toes RE: CD25+ cell depletion hastens the onset of severe disease in collagen-induced arthritis. Arthritis Rheum 2003, 48:1452-1460.

2. Loughry A, Fairchild S, Athanasou N, Edwards J, Hall FC: Inflammatory arthritis and dermatitis in thymectomized $\mathrm{CD}_{25}{ }^{+}$cell depleted adult mice. Rheumatology 2005, in press.

3. Ehrenstein MR, Evans JG, Singh A, Moore S, Warnes G, Isenberg DA, Mauri C: Compromised function of regulatory $T$ cells in rheumatoid arthritis and reversal by anti-TNFalpha therapy. J Exp Med 2004, 200:277-285.

P40

Heme oxygenase 1 regulates osteoclastogenesis

J Zwerina', S Hayer', K Redlich', I Radda', O Hoffmann'², JS Smolen', G Schett ${ }^{1}$

${ }^{1}$ Division of Rheumatology, Department of Internal Medicine III, Medical University of Vienna, Austria; 2Institute for Pharmacy, University of Vienna, Austria Arthritis Res Ther 2005, 7(Suppl 1):P40 (DOI 10.1186/ar1561)

Heme oxygenase 1 (HO-1) plays an important role in vascular disease, transplantation and inflammation. In animal models of acute and chronic inflammation, induction of HO-1 has anti-inflammatory and cytoprotective properties. Since inflammation is an important trigger of osteoclastogenesis, we hypothesized that $\mathrm{HO}-1$ might play a role in osteoclastogenesis.

When HO- 1 was induced by hemin in vitro, a significant dose-dependent inhibitory effect on osteoclastogenesis was observed. Hemin primarily inhibited differentiation of mononuclear osteoclast precusors to osteoclasts. These effects were based on a downregulation of the expression of c-fms and RANK, the receptors for monocyte-colony stimulating factor and RANKL, whereas MAP kinase, NF-KB or Akt signalling were not affected. In vivo, $\mathrm{HO}-1$ induction prevented endotoxin-induced calvarial bone resorption. Furthermore, assessment of synovial tissue from rheumatoid arthritis (RA) patients showed expression of $\mathrm{HO}-1$ in monocytes and fibroblasts, whereas osteoclasts were rarely $\mathrm{HO}-1$ positive. To further assess the role of HO-1 activity in RA patients, we correlated the presence of local bone erosions with serum bilirubin levels. We observed significant higher bilirubin levels in nonerosive than in erosive RA patients. Thus, an increase of HO-1 expression is antiosteoclastogenic in vitro and might protect from increased bone resorption in vivo. Acknowledgement This study was supported by the START price of the Austrian Science Fund (GS).

\section{P41}

How does tumour necrosis factor uncouple T-cell receptorinduced IL-2 gene expression in murine T cells? JM Clark, K Aleksiyadis, M Panesar, AP Cope

The Kennedy Institute of Rheumatology Division, Faculty of Medicine, Imperial College London, UK

Arthritis Res Ther 2005, 7(Suppl 1):P41 (DOI 10.1186/ar1562)

Background T-cell receptor (TCR)-induced IL-2 expression is suppressed the level of mRNA and protein following chronic culture of T cells with tumour necrosis factor (TNF) at picomolar concentrations, an effect that is reversible upon removal of TNF. This TCR hyporesponsiveness is reminiscent of that of T cells recovered from inflamed sites such as the rheumatoid synovium. We have found that, although chronic TNF attenuates TCR $\zeta$ expression and TCR-proximal signalling, IL-2 expression is suppressed regardless of whether cells are stimulated via the TCR, or with phorbol ester and calcium ionophore to activate the MAPK and NFAT pathways directly. Suppression therefore occurs independently of TCR-proximal effects of TNF $[1,2]$.

Objectives We are studying the mechanisms by which chronic TNF suppresses $\mathrm{T}$-cell responses in the $\mathrm{CD}^{+}$mouse $\mathrm{T}$-cell hybridoma clone, 11A2. We have compared the stability of induced IL-2 mRNA, and the regulation of transcription factors important for IL-2 gene expression, in control and TNF-treated cells; and we have recently begun to study effects of chronic TNF on chromatin remodelling across the IL-2 proximal promoter (plL-2), which precedes initiation of transcription, using the chromatin accessibility real-time PCR (CHART-PCR) assay [3]. Methods Cells were grown in the presence or absence of $2.5 \mathrm{ng} / \mathrm{ml}$ murine TNF for 8 days. Washed cells were stimulated with either plate-bound anti-CD3\&, or phorbol 12-myristate 13-acetate (PMA) and ionomycin, for 4 hours. 
$m R N A$ stability assay Actinomycin $\mathrm{D}$ was added and cells harvested over a further 4-hour time-course. Total RNA was extracted and probed for IL-2 mRNA by ribonuclease protection assay.

Transcription factor regulation Whole cell lysates were probed for NF- $\kappa \mathrm{B}$ and IкB family proteins by immunoblot.

Chromatin remodelling Nuclei were isolated and subjected to limited nuclease digestion. DNA was extracted and quantitative analysis of target sequences within plL-2 carried out by real-time PCR. An increase in the threshold cycle $\left(C_{T}\right)$ of a target sequence PCR product from activated cells was indicative of stimulation-induced chromatin remodelling.

Results IL-2 mRNA induced via TCR was unstable $\left(t_{1 / 2}<30 \mathrm{~min}\right)$, whereas PMA and ionomycin stabilised IL-2 mRNA strongly ( $t_{1 / 2}>2$ hours). However, the initial rate of decay was similar in control and TNF-treated cells, suggesting that reduced expression in TNF-treated cells was not due to decreased stability of IL-2 mRNA. $\mathrm{c}-\mathrm{Re} \mathrm{l}, \mathrm{I} \mathrm{KB} \beta$ and $\mathrm{I} \mathrm{B} \varepsilon$ were regulated differently in control and TNF-treated cells: expression of $\mathrm{I} \mathrm{KB} \varepsilon$ was comparatively enhanced, while that of $\mathrm{c}-\mathrm{Rel}$ and $\mathrm{I} \mathrm{B} \beta \beta$ was attenuated in TNF-treated cells. These altered responses may affect the ability of TNF-treated cells to remodel plL-2 productively through NFKB consensus binding upon cell stimulation [4]. This hypothesis is now being tested by CHART-PCR.

Conclusions Chronic culture of murine T cells in TNF does not alter the stability of IL-2 mRNA induced via TCR, or by PMA and ionomycin, in those cells. However, altered regulation of NF-KB expression and activity in TNF-treated cells may contribute to poor inducibility of IL-2 through effects on stimulus-induced changes in chromatin conformation across plL-2.

\section{References}

1. Isomäki P, Panesar M, Annenkov A, Clark JM, Foxwell BM, Chernajovsky Y, Cope AP: Prolonged exposure of T cells to TNF down-regulates TCR zeta and expression of the TCR/CD3 complex at the cell surface. J Immunol 2001, 166::5495-5507.

2. Clark JM, Annenkov AE, Panesar M, Isomäki P, Chernajovsky Y, Cope AP: T cell receptor zeta reconstitution fails to restore responses of $T$ cells rendered hyporesponsive by tumor necrosis factor alpha. Proc Natl Acad Sci USA 2004, 101:1696-1701.

3. Rao S, Procko E, Shannon MF: Chromatin remodeling, measured by a novel real-time polymerase chain reaction assay, across the proximal promoter region of the IL-2 gene. J Immuno/ 2001, 167:4494-4503.

4. Rao S, Gerondakis S, Woltring D, Shannon MF: c-Rel is required for chromatin remodeling across the IL-2 gene promoter. J Immuno/ 2003 , 170:3724-3731.

\section{P42}

The invasiveness of fibroblast-like synoviocytes is of relevance for the rate of joint destruction in patients with rheumatoid arthritis and is a patient characteristic TCA Tolboom ', AHM van der Helm-van Mil', RGHH Nelissen², FC Breedveld', REM Toes', TWJ Huizinga' ${ }^{1}$

${ }^{1}$ Department of Rheumatology, Leiden University Medical Center, Leiden, The Netherlands; '2Department of Orthopedics, Leiden University Medical Center, Leiden, The Netherlands

Arthritis Res Ther 2005, 7(Suppl 1):P42 (DOI 10.1186/ar1563)

Objective Rheumatoid arthritis (RA) is characterized by inflammation of synovial joints and degradation of these joints. Fibroblast-like synoviocytes (FLS) are thought to play a role, because they can invade normal human cartilage in SCID mice [1] and matrigel in vitro [2]. This study was undertaken to investigate the association of these in vitro characteristics with the disease characteristics in patients with RA.

Methods Synovial tissue samples of 72 RA and 50 osteoarthritis patients were obtained; from seven patients with RA, samples of different joints were collected. The FLS invasiveness in Matrigel matrix was studied; the intra-individual and interindividual differences were compared. The radiological scores of the X-rays of the hands and feet of the patients with the FLS that exhibit the most extreme differences in in vitro ingrowth (most invasive and least invasive FLS) were determined with the Sharp-van der Heijde method to determine the relationship between in vitro invasion data and clinical data.

Results FLS from patients with RA are more invasive than FLS from patients with osteoarthritis $(P<0.001)$. The intra-individual variation in FLS invasion was much less than the inter-individual variation $(P=0.028$; mean difference \pm standard deviation, $1204 \pm 926$ and $3476 \pm 2367$ for intra-individual and inter-individual variation, respectively), showing that the level of FLS invasion is a patient characteristic. The mean Sharp score of X-rays of the hands and feet divided by the disease duration was $4.7 \pm 3.2$ units per year of disease duration $(n=9)$ for the patients with the least invasive disease, which was much lower as compared with $22.3 \pm 9.4$ units per year of disease duration $(n=8)$ for the patients with the most invasive disease $(P<0.001)$.

Conclusion The ex vivo behaviour of FLS is a patient characteristic given the destruction in patients with RA. This suggests that the ex vivo invasive behaviour of FLS from patients with RA is of relevance for the rate of joint destruction in patients with RA.

References

1. Muller-Ladner U, Kriegsmann J, Franklin BN, Matsumoto S, Geiler T, Gay RE, Gay S: Synovial fibroblasts of patients with rheumatoid arthritis attach to and invade normal human cartilage when engrafted into SCID mice. Am J Pathol 1996, 149:1607-1615

2. Tolboom TC, Pieterman E, Van Der Laan WH, Toes RE, Huidekoper AL, Nelissen RG, Breedveld FC, Huizinga TW: Invasive properties of fibroblast-like synoviocytes: correlation with growth characteristics and expression of MMP-1, MMP-3, and MMP-10. Ann Rheum Dis 2002 61:975-980.

Acknowledgements TCAT and AHMvdH-vM contributed equally to this work.

P43

A role for IL-7 in regulating $C D 4+C D 25^{\text {high }}$ regulatory $\mathrm{T}$ cells CA Lawson 1,2, AK Brown', SL Field ${ }^{2}$, C Burgoyne ${ }^{2}$, P Emery ${ }^{1}$, JD Isaacs, ${ }^{3}$ F Ponchel1,2

${ }^{1}$ Department of Rheumatology, University of Leeds, UK; ${ }^{2}$ Molecular Medicine Unit, University of Leeds, UK; ${ }^{3}$ Department of Rheumatology, University of Newcastle, UK

Arthritis Res Ther 2005, 7(Suppl 1):P43 (DOI 10.1186/ar1564)

Background Despite the accumulation of evidence that $\mathrm{CD} 4{ }^{+} \mathrm{CD} 25^{\text {high }}$ regulatory $\mathrm{T}$ cells play an important role in the prevention of autoimmune disease, little is known about how they are regulated in vivo. There are thought to be at least two mechanisms for the generation of regulatory $T$ cells: naturally occurring $\mathrm{CD} 4{ }^{+} \mathrm{CD} 25^{\text {high }}$ regulatory $\mathrm{T}$ cells derive from the thymus, and peripherally-induced regulatory cells arise under tolerogenic conditions. Recently, cytokine activation requirements for $\mathrm{CD} 4+\mathrm{CD} 25$ high regulatory $\mathrm{T}$-cell function were associated with IL-7, as well as IL-2 and IL-4. We examined a cohort of patients with rheumatoid arthritis whose disease was well controlled, and where we had previously shown that heterogeneous circulating levels of IL-7 positively correlated with thymic activity, to investigate the role of IL-7 on the function of CD4 ${ }^{+} \mathrm{CD} 25^{\text {high }} \mathrm{T}$ cells.

Methods Peripheral blood samples were taken from patients with rheumatoid arthritis whose disease was well controlled. Serum IL-7 levels were measured by ELISA. Quantification of the CD4+CD25high T-cell subset was performed using flow cytometry. Thymic activity was evaluated by real-time PCR quantification of $\mathrm{T}$-cell receptor excision circles in $\mathrm{CD}^{+} \mathrm{T}$ cells. Thymidine incorporation assays were used to assess the response of $\mathrm{CD} 4^{+} \mathrm{CD} 25^{\text {high }} \mathrm{T}$ cells to IL-7 stimulation, and also their ability to suppress the proliferation of CD4 ${ }^{+} \mathrm{CD} 25^{-} \mathrm{T}$ cells in response to phytohaemagglutinin in co-culture.

Results Circulating levels of IL-7 positively correlated with the frequency of circulating $\mathrm{CD} 4^{+} \mathrm{CD} 25^{\text {high }} \mathrm{T}$ cells $(n=47, R=0.647, P<0.0001)$. This appeared to result from an increased production of these cells by the thymus. High levels of circulating IL-7 in vivo were associated with increased suppressor functions of CD4 ${ }^{+} \mathrm{CD} 25^{\text {high }} \mathrm{T}$ cells in vitro $(n=5$, mean $79 \%$ suppression by cells from patients with high IL-7 levels, and 39\% suppression by cells from patients with low IL-7 levels). In patients with low circulating IL-7 levels, adding exogenous IL-7 to co-cultures appeared to increase suppression.

Conclusions Our data suggest that IL-7 has a role in regulating CD4+CD25 high T-cell number and function. Circulating IL-7 levels are low in active rheumatoid arthritis, and this may be a contributory factor to the reduced size and suppressor function of the CD $4{ }^{+} \mathrm{CD} 25^{\text {high }}$ regulatory T-cell population in this disease.

\section{P44}

\section{Results of the Consensus Finding Study Group on} Autoantibodies (March 2003-March 2004)

\section{Hamann', P Charles' ${ }^{2}$ M Renz ${ }^{3}$, HP Seelig ${ }^{3}$, A Wiik4, RJT Smeenk'}

${ }^{1}$ Department of Autoimmune Diseases, Sanquin Diagnostic Services at CLB, Amsterdam, The Netherlands; ' ${ }^{2}$ Department of Rheumatology, Kennedy Institute and Hammersmith Hospitals, London, UK; ${ }^{3}$ Laboratory Prof. HP Seelig, Dr R

Seelig and Colleagues, Karlsruhe, Germany; ${ }^{4}$ Department of Autoimmunology, Statens Serum Institut, Copenhagen, Denmark

Arthritis Res Ther 2005, 7(Suppl 1):P44 (DOI 10.1186/ar1565)

The European Consensus Finding Study Group on Autoantibodies is currently formed by 43 European laboratories involved in the field of serological diagnostics in rheumatic diseases. The aim of this group is to work towards achieving common consensus in this field: a laboratory result should be the same, wherever the result is obtained.

The Steering Committee of the Consensus Finding Study Group tested 24 candidate sera that had been contributed by the participating laboratories. Ten sera were selected for the annual serum round. This year, special emphasis was put on anti-DNA antibody testing. For this purpose four sera were included that contained different specificities of anti-DNA (Table 1). The remaining six sera were positive for a variety of autoantibodies (Table 1) 
Table 1

\section{Summary of results for $\mathbf{1 0}$ distributed sera}

\begin{tabular}{lll}
\hline Serum & Detected antibodies & Comments \\
\hline 1 & dsDNA & Only Crithidia luciliae positive \\
2 & SS-A $52 \mathrm{kDa}+60 \mathrm{kDa}, \mathrm{SS}-\mathrm{B}$ & Good consensus \\
3 & SS-A $52 \mathrm{kDa}, \mathrm{SCL}-70$ & SS-A $60 \mathrm{kDa}$ only found by minority \\
4 & PR-3 ANCA & Very few incorrect results \\
5 & SS-B & SS-A found by ELISA, \\
& & counterimmunoelectrophoresis and \\
6 & U1-snRNP, A and C protein & immor neglot mostly negative \\
7 & Centromere (CENP-B) & Good consensus \\
8 & ssDNA & Perfect consensus \\
9 & dsDNA & No consensus \\
10 & dsDNA & Good consensus \\
\hline
\end{tabular}

For the first time, a dedicated computer program was used to allow more easy data entry and statistical analysis. Next to the 10 sera, a CD-ROM containing 20 HEp-2 cell staining patterns was dispatched to the participating laboratories. The CD-ROM also contained the spreadsheets to be filled in by the participants.

Results were returned by 38 out of 43 participating laboratories. The data were discussed at a satellite meeting during the 24th European Workshop on Rheumatology Research in Berlin and were published on the website of the Sanquin Blood Supply Foundation in Amsterdam (via a link distributed to the participants). In depth analysis of individual results compared with the consensus were sent to the participants.

In general, the participants reached a high degree of consensus for most of the samples. The results for anti-DNA were very promising. A perfect consensus was reached for the absence of dsDNA antibodies in an anti-ssDNA specific serum (serum 8 ). The absence of false positive results showed that assays measuring anti-dsDNA antibodies have been improved such as to exclude detection of antissDNA. Serum 9 was obtained from a rheumatoid arthritis patient who had been treated with anti-tumour necrosis factor alpha (Infliximab) and developed antidsDNA antibodies, yet showed no clinical signs of systemic lupus erythematosus. Using different assays, divergent results were obtained. Most laboratories found anti-dsDNA antibodies by the indirect immunofluorescence technique (substrate Crithidia (uciliae), whereas anti-dsDNA ELISAs were negative. For this sample most (commercial) Farr assays showed positive reactions. Detailed analysis of the serum in Amsterdam showed the presence of low avidity $\lg M$ and IgG antibodies to dsDNA. In order to understand the cause of the discrepancies the influence of choice of assay will be studied in more detail. Divergent results, depending on the method for detection, were also found for antibodies to SS-A. Obviously, ELISA-based techniques were more sensitive. Unfortunately, most ELISAs do not distinguish between anti-SS-A 52 and anti-SS-A 60 antibodies. The clinical relevance of the lower limit of detection has to be established in the future. We will include a more detailed method analysis in the next rounds.

There was general agreement among the participating laboratories that these Consensus Rounds should be continued, including the HEp-2 cell pattern recognition CD-ROM effort. It was decided to focus next round on anti-phospholipid antibodies. For information about the study group please contact Ruud Smeenk (r.smeenk@sanquin.nl).

\section{P45}

Effects of overexpression of PAD4 enzyme in mouse synovium AJW Zendman', WAM Horstman', AJ Arntz'2, MB Bennink2, ER Vossenaar', WJ van Venrooij', WB van den Berg'2, FAJ van de Loo'2, GJM Pruijn'

${ }^{1}$ Department of Biochemistry 161, Radboud University Nijmegen, The Netherlands; ${ }^{2}$ Department of Experimental Rheumatology, University Medical Center St Radboud, Nijmegen, The Netherlands

\section{Arthritis Res Ther 2005, 7(Suppl 1):P45 (DOI 10.1186/ar1566)}

Background Autoantibodies directed against citrullinated proteins (e.g. antiCCP) can be detected in rheumatoid arthritis (RA) patients with very high specificity. The antibodies are present already years before the first clinical symptoms and their presence predicts the development of erosive disease. Citrullinated proteins, the target of anti-CCP antibodies, are formed by post-translational deimination of arginine residues, catalyzed by peptidylarginine deiminase enzymes (PADs). PAD enzymes are expressed by cells present in the inflamed joints of RA patients. These data are the basis of our hypothesis that protein citrullination by PAD is intimately involved in the development of RA.
Objective To investigate the effect of PAD4 overexpression in synovium of naive mice and in mouse models of arthritis.

Methods An adenoviral delivery tool for local expression of mouse PAD4 was successfully constructed. Using this adenoviral construct, PAD4 overexpression was effectuated in cells present in the synovial cavity after intraarticular injection. Effects of PAD4 overexpression in mice with or without co-induction of arthritis (streptococcal cell wall arthritis) were investigated using routine scoring of arthritic features (e.g. swelling) and histology. The presence of citrulline-specific antibodies was measured by ELISA using a synthetic citrullinated peptide (CCP1) and a noncitrullinated control peptide.

Results In all naive mice (C57/BI6, $n=12$ ) we were able to effectively overexpress PAD4 in the synovial tissue, lasting at least 1 week. This overexpression resulted in the generation of citrullinated proteins in vivo. In mice receiving a control adenovirus no citrullinated proteins were observed. The overexpression did not induce inflammation. Similar experiments in streptococcal cell wall (SCW)-induced mice (C57/BI6 + SCW, $n=8$ ) showed a prolonged expression of citrullinated proteins when the knees were preloaded with PAD4 (compared with control adenovirus-treated SCW mice, $n=8$ ), but this did not affect the level of inflammation or tissue damage. Overexpression of PAD4 did not lead to the production of citrulline-specific autoantibodies in these mice.

Conclusion Adenoviral expression constructs can successfully be used to introduce PAD enzymes in mouse synovium. Overexpression of PAD4 results in the production of synovial citrullinated proteins, but did not cause additional inflammation in mice nor did it cause an immune response to citrullinated proteins. Generating anti-CCP-positive mice will be crucial in studying the effect of specific immune complexes on arthritic phenomena in mouse models of arthritis. Acknowledgements This work was financially supported by the Netherlands Foundation for Chemical Research and the Netherlands Technology Foundation, the Dutch League against Rheumatism and the Netherlands Foundation for Medical Research.

\section{P46}

Long-term survival after lymphocytotoxic monoclonal antibody therapy for rheumatoid arthritis JD Isaacs' ${ }^{1}$ AM Clarke², BL Hazleman ${ }^{3}$, G Hale ${ }^{4}$, H Waldmann ${ }^{4}$, DPM Symmons ${ }^{2}$

${ }^{1}$ School of Clinical Medical Sciences, University of Newcastle upon Tyne, UK; ${ }^{2}$ ARC Epidemiology Unit, University of Manchester, UK; ${ }^{3}$ Department of Rheumatology, Addenbrooke's Hospital NHS Trust, Cambridge, UK;

${ }^{4}$ Dunn School of Pathology, University of Oxford, UK Arthritis Res Ther 2005, 7(Suppl 1):P46 (DOI 10.1186/ar1567)

Background In the early 1990s, we used the monoclonal antibody alemtuzumab (MabCampath, Schering AG, Berlin, Germany) to treat patients with refractory rheumatoid arthritis. This treatment provided temporary relief of symptoms but was associated with long-term lymphopenia, particularly of T lymphocytes [1]. We continue to follow these patients to exclude any adverse effects of their longterm lymphopenia.

Objective The objective of the current study was to compare 10-year mortality in this patient cohort with mortality in a control patient cohort.

Methods Fifty-three rheumatoid arthritis patients that received alemtuzumab (median dose, 172 mg; range, 1-420 mg) between 1991 and 1994 (cases) were monitored via the Office for National Statistics Central Registry, to ensure notification of death. A retrospective, matched-cohort study of mortality was performed with 102 control subjects selected from the European League Against Rheumatism database. This database comprises patients with rheumatic disorders who have received immunosuppressive drugs, usually cyclophosphamide or azathioprine.

Results Median (range) duration of follow-up was 10.29 (1.27-12.15) years for cases and $10.35(1.18-12.14)$ years for controls. There were 20 deaths among the cases compared with 37 among the controls. This provided a mortality rate of 0.045 deaths per person per year for the cases and 0.041 deaths per person per year for the controls. The mortality rate ratio was 1.10 (95\% confidence interval, $0.61-1.95)$. There was no significant difference in survival between the two groups ( $P=0.73$, log-rank test). Figure 1 illustrates Kaplan-Meier survival plots for cases and controls. The causes of death in the cases reflected those expected in a hospital-based rheumatoid arthritis cohort. Median time (range) from treatment to death for cases was $5.9(1.3-11.7)$ years. Mortality did not differ according to total dose of alemtuzumab or the number of courses received. Conclusions Despite long-term lymphopenia, there was no excess mortality in recipients of alemtuzumab at a median follow-up of 10.3 years from treatment.

Reference

1. Isaacs JD, Greer S, Sharma S, Symmons D, Smith M, Johnston J, Waldmann $\mathrm{H}$, Hale G, Hazleman BL: Morbidity and mortality in rheumatoid arthritis patients with prolonged and profound therapy-induced lymphopenia. Arthritis Rheum 2001, 44:1998-2008.

Acknowledgements This work was supported by llex Oncology, Inc. and the Arthritis Research Campaign 
Figure 1

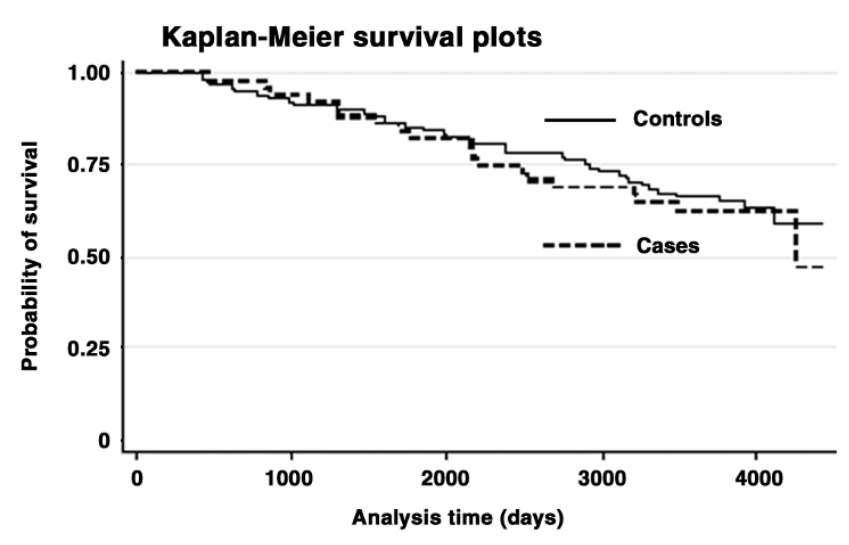

Kaplan-Meier survival plots.

\section{P47}

The persistence of abnormal T-cell differentiation patterns predicts relapse in rheumatoid arthritis patients with controlled disease

\section{A Brown ${ }^{1}$, F Ponchel' ${ }^{1}$ J Isaacs ${ }^{2}$, P Emery ${ }^{1}$}

${ }^{1}$ University of Leeds, UK; ${ }^{2}$ University of Newcastle, UK

Arthritis Res Ther 2005, 7(Suppl 1):P47 (DOI 10.1186/ar1568)

Background We previously demonstrated abnormal T-cell differentiation in rheumatoid arthritis (RA) and hyperproliferation of T cells in relation with inflammation. However, it is still not clear whether these T-cell dysfunctions are a primary feature of RA or are only secondary to inflammation. We therefore analysed RA patients in clinical remission in whom systemic inflammation was controlled.

Methods Patients were defined as in remission when they had controlled disease, with no change of activity for at least 6 months, C-reactive protein below 15 , no swollen or tender joints and on stable treatment. Relapse was defined as a change in disease activity sustained for at least 3 months, requiring or not a change in treatment. Flow cytometry was used to assess T-cell differentiation pattern in controls $(n=23)$, active RA $(n=28)$ and remission $(n=34)$. CD4 $4^{+} \mathrm{T}$ cell proliferation assay in response to mitogen, IL-2, and antibody stimulation in eight paired blood samples for active and remission disease. Statistical analysis was used to seek clinical and laboratory correlate to differentiation abnormalities. Results The abnormal differentiation patterns observed in active disease were maintained in remission. The first inflammation-associated subset was persistent. The second atypical subset was never observed in health, poorly represented in active disease but largely accumulated in remission. The hypreproliferation associated with the first subset was lost in remission and was therefore driven by inflammation. The second atypical subset was associated with profound hyporesponsiveness in both active and remission disease. Relapse occurred in 13 out of 32 patients within 12 months of follow-up. Relapse was associated with a higher frequency of the inflammation-related cells $(P=0.017)$. We proceeded to a complete analysis of factors that could predict relapse. Only rheumatoid factor positivity $(P=0.033)$ and possibly having nodules $(P=0.079)$ were associated with relapse. Using a regression analysis revealed that the frequency of inflammation related cells was highly significant in predicting relapse, being correct in $78 \%$ of cases $(P=0.025)$. Neither the presence of rheumatoid factor or nodule improved this model.

Conclusions These results suggest that the hyper-reactivity of the inflammatory subset is associated with disease activity; however, its persistence in the absence of inflammation suggests a primary defect possibly in activation-induced cell death. The second subset appear fairly inactive, but more work is necessary to fully characterise these cells. Its complete absence in health and accumulation in disease remission also suggests a primary defect in T-cell differentiation pathways in RA.
P48

The inhibitor of differentiation-2 promotes synovial fibroblast-dependent osteoclastogenesis in rheumatoid arthritis

M Kurowska-Stolarska ${ }^{1}$, J Distler', W Rudnicka², E Neumann ${ }^{3}$, T Pap ${ }^{4}$, R Wenger ${ }^{5}$, A Jungel', BA Michel', U Muller-Ladner ${ }^{3}$, RE Gay', W Maslinski ${ }^{2}$, $\mathbf{S}$ Gay ${ }^{1}$, O Distler $^{1}$

${ }^{1}$ Center of Experimental Rheumatology, University Hospital, Zurich, Switzerland; ${ }^{2}$ Department of Pathophysiology and Immunology, Institute of Rheumatology, Warsaw, Poland; ${ }^{3}$ Department of Internal Medicine, University of Regensburg, Germany; ${ }^{4}$ Division of Experimental Rheumatology, Otto von Guericke University, Magdeburg, Germany; 5 Institute of Physiology, University of Zurich, Switzerland Arthritis Res Ther 2005, 7(Suppl 1):P48 (DOI 10.1186/ar1569)

Objectives Despite indirect evidence suggesting that low oxygen levels might occur in the rheumatoid arthritis (RA) synovium, direct proof of the presence of hypoxia in the arthritic synovium as well as the relevance of low oxygen levels for joint destruction is lacking. The aim of this study was to analyse the distribution of hypoxia in arthritic joints and to evaluate the molecular effects of the hypoxic environment on the phenotype of RA synovial fibroblasts (SF).

Methods The hypoxia marker EF-5 was applied in mice with the collagen-induced arthritis (CIA). Expression profile analysis with hypoxic and normoxic SF was performed using subtractive hybridization and microarray. The expression of the inhibitor of differentiation-2 (Id-2), CD68 (macrophage marker) and prolyl hydroxylase (fibroblast marker) was evaluated by immunohistochemistry on synovial tissues from RA, osteoarthritis patients and ClA mice. To evaluate the function of Id-2 in SF, cells were transfected with the pcDNA3.1 containing cDNA for Id-2 or Id-2-specific siRNA or mock controls. The expression of Id-2 and genes regulated by Id-2 in transfected SF was evaluated by SYBR Green real-time PCR and western blot. SF stably transfected with Id-2 were cocultured with bone marrow cells in a transwell system. The expression of the receptor activator of NF- $\mathrm{kB}$ ligand (RANKL) and osteoprotegerin were measured by real-time PCR. The development of osteoclasts was evaluated by visualization of the activity of tartrate-resistant acid phosphatase.

Results Using the hypoxia marker EF-5 we found that in mice with CIA, synovial cells invading bone and cartilage are exposed to reduced oxygen levels. Expression profile studies identified Id-2 as being upregulated under low oxygen conditions. In addition, IL-1beta stimulation increased the expression of Id-2 in these cells. Histological studies of RA synovium and CIA synovium showed strong expression of Id-2 in SF at sites of synovial invasion into bone. Overproduction of Id-2 in SF by stable transfection triggered the expression of several genes promoting osteoclastogenesis, including BMP-2, PTHrP, Wnt5a and vascular endothelial growth factor. Conversely, the suppression of endogenous ld-2 led to the downregulation of the expression of these molecules. Consistent with these findings coculture of Id-2 transfected SF with bone marrow cells increased the expression of the osteoclast differentiation factor RANKL, and decreased the expression of the osteoclast inhibitory factor osteoprotegerin in bone marrow stromal cells, which was followed by an increase in the number of osteoclasts. Conclusion Taken together, our data provide evidence that hypoxia is present at sites of synovial invasion in RA and that Id-2 induced by hypoxia contributes at these sites to joint destruction by promoting SF-dependent osteoclastogenesis.

P49

Abstract withdrawn 
P50

Identification of the EGF-TM7 receptor EMR2 and its ligand dermatan sulphate in rheumatoid synovial tissue EN Kop1, MJ Kwakkenbos'2, GJD Teske1, MC Kraan', TJ Smeets', M Stacey ${ }^{3}$, H-H Lin ${ }^{3}$, J Hamann' ${ }^{2}$, PP Tak ${ }^{1}$

${ }^{1}$ Division of Clinical Immunology and Rheumatology, Academic Medical Center, Amsterdam, The Netherlands; ${ }^{2}$ Laboratory of Experimental Immunology, Academic Medical Center, Amsterdam, The Netherlands; ${ }^{3}$ Sir William Dunn School of Pathology, University of Oxford, UK

Arthritis Res Ther 2005, 7(Suppl 1):P50 (DOI 10.1186/ar1571)

Introduction EMR2 is a member of the EGF-TM7 family closely related to CD97. Chondroitin sulphates have recently been identified as ligands for EMR2 and CD97. Chondroitin sulphates have been implicated in the pathogenesis of rheumatoid arthritis (RA). The objective of this study is to determine the expression of EMR2 and the distribution of EMR2 and CD97 ligands in RA synovial tissue.

Methods Synovial tissue samples were obtained by arthroscopy from patients with RA $(n=19)$, inflammatory osteoarthritis $(n=13)$, and reactive arthritis $(n=13)$. Immunohistologic staining was performed with EMR2 mAb and stained synovial tissue sections were analysed by digital image analysis. Co-expression of EMR2 with lineage and activation markers was determined by double immunofluorescence microscopy. To evaluate the expression of EMR2 and CD97 ligands in RA synovium, binding assays using fluorescent beads loaded with EMR2-Fc or CD97-Fc constructs were performed.

Results EMR2 expression in the synovial sublining was significantly higher in RA compared with disease controls. Most EMR2-positive cells were macrophages and dendritic cells, expressing co-stimulatory molecules and tumour necrosis factor alpha. Dermatan sulphate was shown to be the ligand of the largest isoform of EMR2 and CD97 in rheumatoid synovium. In addition, the smaller isoforms of CD97, but not EMR2, bound CD55 on fibroblast-like synoviocytes. Conclusion The EGF-TM7 receptor EMR2 and CD97 are abundantly expressed on myeloid cells in synovial tissue of RA patients where they encounter their ligands dermatan sulphate and CD55. These interactions might facilitate the retention of activated macrophages in the synovium.

\section{P51}

Prevalence and specificity of autoantibodies to defensins in sera of patients with systemic autoimmune diseases AG Terzoglou, JG Routsias, AG Tzioufas, HM Moutsopoulos

Department of Pathophysiology, School of Medicine, University of Athens, Greece Arthritis Res Ther 2005, 7(Suppl 1):P51 (DOI 10.1186/ar1572)

Background Defensins are small positively charged peptides (29-47 amino acids) that are highly conserved among species and play a role in host defense against bacteria and viruses. There are two categories of defensins: A-defensins are secreted from human neutrophils, and B-defensins are produced from the epithelial cells. Each peptide contains six cystein amino acid residues, connected with disulfide bonds. The physicochemical properties of defensins are similar with the properties of another molecule, B2-glycoprotein I (B2-GPI), the major target of the autoantibodies found in the sera of patients with antiphospholipid syndrome (APS). Objective We investigated whether sera from patients with APS as well as primary Sjogren Syndrome (pSS), systemic lupus erythematosus (SLE), and rheumatoid arthritis $(\mathrm{RA})$ contain autoantibodies that react with defensins.

Methods We synthesized the linear peptides $\mathrm{NH}_{2}$-ACYCRIPACIAGERRYGTCIYOGRLWAFCC-OH and $\mathrm{NH}_{2}$-GIGDPVTCLKSGAICHPVFCPRRYKOIGTCGLPGTKCCKKP-OH, corresponding to the amino acid sequences of the mature forms of $A 1$ (defA1) and B2 (defB2) defensins, respectively. These peptides were oxidized, in order for the disulfide bonds of the native molecules to be created, and subsequently were lyophilized. The peptides were dissolved in water and they were tested in ELISA experiments against sera from patients with APS $(n=24)$, pSS $(n=24)$, SLE $(n=16)$, and RA $(n=8)$. Additionally, sera from normal individuals were tested. Homologous inhibition experiments were performed in order to examine the specificity of the immune response against defensins.

Results None from the tested sera reacted against the defA1. Sera from patients with systemic autoimmune diseases contained autoantibodies to defB2 as follows: $21 \%$ of patients with APS and $25 \%, 31 \%$, and $12 \%$ of the sera from the patients with $\mathrm{pSS}, \mathrm{SLE}$, and RA, respectively, gave a positive reaction against the same peptide. None of the normal sera reacted with the peptides at all. In the inhibition experiments the defB2 peptide, when it was used as soluble inhibitor, inhibited the binding of the antibodies at the plate-bound defB2 by $64 \%$.

Discussion Defensins are components of the innate immunity and share common physicochemical properties with the B2-GPI molecule. A rather small proportion of sera from patients with systemic autoimmune diseases contain antibodies that react specifically with the defB2 peptide. The presence of these autoantibodies is not disease specific and their pathogenic significance, if any, remains to be elucidated.
P52

Tumor necrosis factor alpha reverses the immunosuppressive properties of mesenchymal stem cells in collagen-induced arthritis

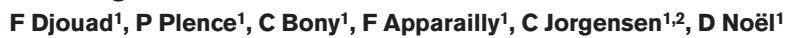

${ }^{1}$ INSERM U475, Montpellier, France; ${ }^{2}$ Department of Immuno-rheumatology, Lapeyronie Hospital, Montpellier, France

Arthritis Res Ther 2005, 7(Suppl 1):P52 (DOI 10.1186/ar1573)

Background Mesenchymal stem cells (MSCs) are bone-marrow-derived progenitor cells, widely investigated for their potential of differentiation towards multiple lineages, such as osteocytes and chondrocytes. Recently, we and others showed that MSCs exhibit immunosuppressive properties inducing in vivo tolerance of T lymphocytes towards allogeneic cells. Moreover, these cells can be easily genetically modified to express ectopic molecules, such as anti-inflammatory cytokines. Objective Here, we investigated whether naïve and IL-10-expressing MSCs could display an inhibitory effect towards self-reactive T lymphocytes in vitro and have a biological effect in the murine model of collagen-induced arthritis (CIA).

Methods We used the murine C3H10T1/2 cell line (C3 MSCs) in the CIA murine model of RA. We injected various doses of MSC at immunization or at boost and evaluated the clinical and immunological parameters. Immunosuppressive properties of MSC were determined in vitro in mixed lymphocyte reactions. Results We evaluated the potential immunosuppressive role of MSCs in the CIA model and showed that MSCs did not display any benefit whatever the day of cell injection (day 0 or day 21 ) and the numbers of MSCs $\left(10^{6}\right.$ or $\left.4 \times 10^{6}\right)$. Both clinical and immunological analysis argued for the accentuation of the Th1 helper response. Using luciferase-expressing MSCs, we were unable to detect labeled cells in the articular environment of the knee, suggesting that worsening of the symptoms was unlikely due to the homing of MSCs in the joints. Experiments in vitro showed that addition of tumor necrosis factor alpha was sufficient to reverse the immunosuppressive effect of MSCs on T-cell proliferation and this observation was associated with an increase of IL-6 secretion.

Conclusion Here, we demonstrated that the immunosuppressive properties of MSCs did not provide any clinical benefit in CIA. Our data suggest that environmental parameters, in particular inflammation, may influence the immunosuppressive properties of MSCs.

\section{P53}

Neutralizing IL-17 during re-activation of experimental arthritis prevents joint inflammation and bone erosion by decreasing RANKL and IL-1

\section{Koenders, E Lubberts, LAB Joosten, WB van den Berg}

Department of Rheumatology, Experimental Rheumatology and Advanced Therapeutics, Radboud University Medical Center Nijmegen, The Netherlands Arthritis Res Ther 2005, 7(Suppl 1):P53 (DOI 10.1186/ar1574)

Background Rheumatoid arthritis is characterized by an intermittent course of the disease with alternate periods of remission and relapse. T cells, and in particular the T-cell cytokine IL-17, are expected to be involved in this flare-up of arthritis.

Objective To study the role of T-cell IL-17 in flare-up of experimental arthritis. Methods Antigen-induced arthritis was induced in C57BI/6 mice by immunizing and boosting with mBSA/ complete Freund's adjuvant, and subsequent intraarticular injection of $60 \mu \mathrm{g}$ mBSA. At week 4 of arthritis, $2 \mu \mathrm{g} \mathrm{mBSA}$ was injected into the arthritic joint to induce a flare-up of the smouldering inflammation. To study the role of IL-17 in this flare-up, neutralizing rabbit-anti-mouse-IL-17 antibodies (or control antibodies) were injected 2 hours prior to antigen rechallenge. Results Quantitative PCR at various time points after arthritis induction showed that IL-17 mRNA expression was already upregulated at day 1 , increased even more at day 2 and day 7 , and clearly diminished at day 21. After antigen rechallenge, IL-17 mRNA expression rapidly increased, peaking at 4 hours with a 250fold upregulation compared with naive mice.

Neutralizing IL-17 significantly prevented joint swelling, as measured by ${ }^{99 \mathrm{~m} T \mathrm{C}}$ uptake at day 1 (Fig. 1a). Arthritic knee joints were isolated at day 4 , and histological analysis showed significantly suppressed joint inflammation (Fig. 1b) and cartilage proteoglycan depletion in the anti-IL-17-treated group.

Blocking IL-17 also clearly protected arthritic mice against bone erosions (Fig. 1c). Cathepsin K staining showed reduced osteoclast-like activity, and quantitative PCR showed reduced RANKL mRNA expression in the anti-IL-17-treated group. The degree of bone erosions strongly correlated with the severity of joint inflammation, suggesting that neutralizing IL-17 reduces bone erosion by suppressing joint inflammation.

Interestingly, mice treated with anti-IL-17 antibodies showed reduced protein levels of IL-1 $\beta$, tumor necrosis factor alpha, and the cytokine-induced neutrophil chemoattractant KC, suggesting that IL-17 acts as an upstream mediator in destructive joint inflammation during flare-up of experimental arthritis. 
Figure 1
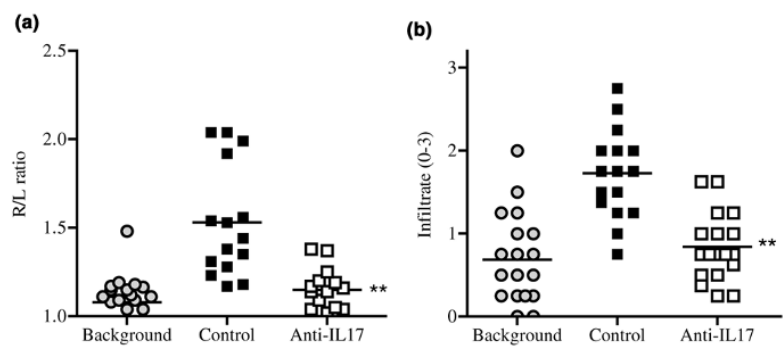

(c)

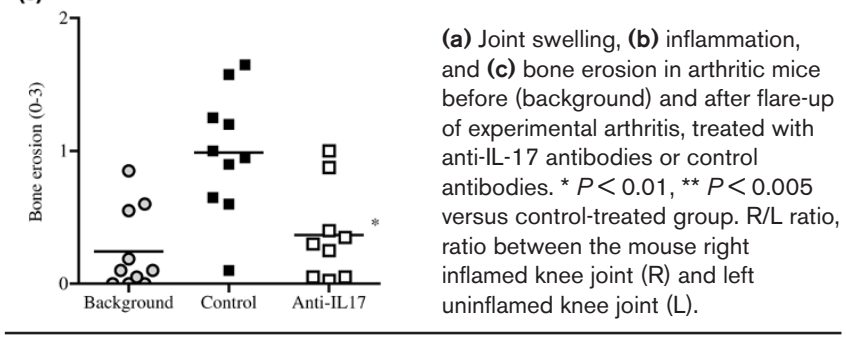

Conclusion These data indicate IL-17 to be an important upstream proinflammatory cytokine driving joint pathology during flare-up of experimental arthritis and suggest therapeutic benefit of neutralizing IL-17 during relapses of rheumatoid arthritis.

\section{P54}

Functional Toll-like receptor 9 modulates the activity of bone marrow $B$ cells isolated from rheumatoid arthritis patients W Rudnicka', E Warnawin', M Buler', T Burakowski', M Bik', E Kontny', C Michalak ${ }^{2}$, P Maldyk ${ }^{2}$, W Maslinski ${ }^{1}$

${ }^{1}$ Department of Pathophysiology and Immunology, Institute of Rheumatology, Warsaw, Poland; ${ }^{2}$ Clinic of Othopedic Surgery, Institute of Rheumatology, Warsaw, Poland

Arthritis Res Ther 2005, 7(Suppl 1):P54 (DOI 10.1186/ar1575)

Background Toll-like receptors (TLRs), a family of pathogen recognition receptors, represent an important component of the innate immune system that also contributes to the development of acquired immunity. TLR9 expressed in the cytoplasm of several cell types including B cells, and recognize and are activated by unmethylated CpG-rich, pathogen-derived DNA sequences. This CpG stimulation triggers B-cell proliferation and promotes Th1 response. Moreover, DNA containing $\mathrm{CpG}$-rich motifs, acting as polyclonal stimuli, participates in the maintenance of serological memory by human memory B cells. Recent data indicate that bone marrow in rheumatoid arthritis (RA) patients may actively participate in the pathogenesis of RA as a secondary lymphoid organ via overproduction of proinflammatory cytokines and a site of effective antigen presentation.

Objective To test the hypothesis that RA bone-marrow-derived B cells express functional TLR9.

Methods Bone marrow mononuclear cells (BMMC) were isolated from RA bone marrow samples obtained during joint replacement surgery. The expression of TLR9 protein on B lymphocytes, gated as CD19-positive cells in BMMC preparation, was assessed by intracellular staining and flow cytometric analysis. BMMC were stimulated in vitro with agonistic (CpG-ODN) or control (GpC-ODN) oligodeoxynucleotides $(15-30 \mu \mathrm{g} / \mathrm{ml})$. In blocking experiments chloroquine $(2-3 \mu \mathrm{g} / \mathrm{ml})$ was added to the culture $50 \mathrm{~min}$ before stimuli. The expression of activation markers CD86 and CD54 on B lymphocytes (CD19+) were analyzed after 48 and 72 hours in culture using flow cytometry. Ki-67 and CFSE staining (flow cytometry) was applied to evaluate B-cell proliferation after 72 and 120 hours of culture with oligodeoxynucleotides. The concentrations of oligodeoxynucleotides and chloroquine used in experiments were not toxic to BMMC as judged by colorimetric lactate dehydrogenase assay. The presence of bacterial DNA in bone marrow plasma and BMMC was evaluated by DNA extraction and PCR amplification. Primers used for PCR recognize highly conserved regions of the eubacterial 16S-ribosomal RNA gene.
Results RA bone-marrow-derived B lymphocytes express TLR9 protein that could be detected via intracellular staining and flow cytometric analysis. Importantly, these TLR9 are functional. CpG-ODN, but not control GpC-ODN, in a dose-dependent manner enhanced the expression of activation markers (CD86 and CD54) on B cells in BMMC cultured in vitro. The specificity of CpG-ODN triggered expression of CD86 was confirmed in experiments where cells cultured in the presence of chloroquine, a known inhibitor of TLR9-triggered signal tranduction, failed to respond to CpG-ODN. Moreover, CpG-ODN triggers bone marrow B-cell proliferation in vitro, as judged by enhanced expression of proliferation marker Ki-67 and a diminished level of CFSE dye. Interestingly, our preliminary data indicate the presence of bacterial DNA in several samples of bone marrow tissues isolated from RA patients. We could amplify DNA encoding bacterial 16S-ribosomal RNA in one bone marrow plasma and three BMMC out of total five analyzed samples.

Conclusions Our results indicate that CpG oligodeoxynucleotides, the potent agonists of TLR9, modulate the activity of $B$ cells from bone marrow of RA patients via induction of costimulatory and adhesion molecules (CD86, CD54) expression, and via cell proliferation. Thus, our data suggest that bone marrow of RA patients may represent an important secondary lymphoid organ that actively participates in the pathogenesis of RA.

\section{P55}

\section{BiP induces IL-4-dependent regulatory cells}

R Brownlie ${ }^{1}$, LK Myers ${ }^{2}$, VM Corrigall ${ }^{3}$, MD Bodman-Smith ${ }^{3}$, SJ Thompson', GS Panayi ${ }^{3}$

'Department of Immunology, School of Life Sciences, KCL, London, UK; ${ }^{2}$ Department of Paediatrics UT Medical Group Inc., Memphis, Tennessee, USA; ${ }^{3}$ Department of Academic Rheumatology, GKT School of Medicine, KCL, London, UK

Arthritis Res Ther 2005, 7(Suppl 1):P55 (DOI 10.1186/ar1576)

Background Previously we have described the stress protein BiP as a putative autoantigen in RA [1]. The administration of BiP intravenously (i.v.) prior to induction of collagen-induced arthritis $(\mathrm{CIA})$ resulted in almost complete amelioration of disease [1]. Our studies now indicate that BiP has several immunomodulatory actions including the skewing of T-cell differentiation towards Th2 [2].

Aim This study focused on the therapy of ClA and investigated subcutaneous administration of $\mathrm{BiP}$ in comparison with intravenous administration and whether adoptive transfer of BiP-treated cells could successfully inhibit the onset of disease. Finally, IL-4 knockout mice were used to determine the importance of the T cell in the therapeutic role of BiP

Methods ClA was induced in DBA-1 mice by injection of bovine collagen type II (CII) in complete Freund's adjuvant followed by a booster injection in incomplete Freund's adjuvant at day 21. At the first appearance of swollen joints, the mice were injected subcutaneously (s.c.) with BiP, a control protein, BSA or vehicle control (PBS). Disease progression was followed by measurement of swollen joints. At termination, splenocytes and draining lymph node cells were removed and T-cell cytokine secretion was assessed. Mixed spleens and lymph nodes from groups of DBA-1 mice that had been immunized s.c. with BiP or BSA $(200 \mu \mathrm{g})$, or i.v. with BiP or BSA $(10 \mu \mathrm{g})$, were collected 12 days after immunization. Cell cultures $\left(2.5 \times 10^{6} \mathrm{cells} / \mathrm{ml}\right)$ were set up with $20 \mu \mathrm{g}$ of the respective protein (BiP or BSA) for 5 days. Cells were then washed and injected intraperitoneally into DBA $/ 1$ mice $\left(20 \times 10^{6}\right.$ cells/mouse $)$ that had received the first Cll immunization 24 days previously. DR $1^{+/+} \mathrm{IL}-4$ knockout mice, $(n=20)$ developed by backcrossing $\mathrm{C} 57 \mathrm{BI} / 6 \mathrm{IL} 4^{-/-}$mice to $\mathrm{DR} 1^{+/+}$mice, were immunized with $\mathrm{Cll}$, and on day 24 after immunization were given $10 \mu \mathrm{g}$ BiP i.v. Wild-type HLADR $1^{+/+}$transgenic mice ( $n=20 /$ group) were administered $10 \mu \mathrm{g} \mathrm{BiP}$ or PBS i.v. Results Administration i.v. or s.c. of BiP significantly reduced $(P<0.05)$ the incidence and severity of CIA when given at the onset of disease. A lower incidence of arthritis was also recorded from groups of mice treated with BiP s.c. and i.v. $(37.5 \%$ and $64 \%$, respectively) as compared with $100 \%$ recorded from the control group by day 70 . T cells removed from mice that had been treated with $\mathrm{BiP}$ via both routes were shown to secrete IL-4 in response to in vitro BiP stimulation. In response to $\mathrm{Cll}$, results indicated upregulation of IL-5 and IL-10 production from BiP-treated groups compared with the arthritic control group. In the adoptive transfer studies the mice receiving subcutaneous BiP-primed cells had a significant suppression of arthritis by day 48 , and by day 66 in those receiving intravenous BiP-primed cells, compared with mice that had received BSA-primed T cells $(P<0.05)$. When the IL- $4^{-/-}$mice were scored for disease severity the $\mathrm{IL}-4^{-I-}$ mice treated with BiP were no different from wild-type mice treated with PBS whereas wild-type mice treated with BiP had a significant suppression of arthritis ( $P \leq 0.05$, Students test). 
Conclusion These findings suggest that treatment of $\mathrm{ClA}$ with $\mathrm{BiP}$ is mediated at least in part by induction of IL-4-dependent regulatory T cells.

References

1 Corrigall VM, Bodman-Smith MD, Fife MS, Canas B, Myers LK, Wooley P, Soh C, Staines NA, Pappin DJ, Berlo SE, et al.: The human endoplasmic reticulum molecular chaperone $\mathrm{BiP}$ is an autoantigen for rheumatoid arthritis and prevents the induction of experimental arthritis. J Immunol 2001, 166:1492-1498.

2. Bodman-Smith MD, Corrigall VM, Kemeny DM, Panayi GS: BiP, a putative autoantigen in rheumatoid arthritis, stimulates IL-10-producing CD8positive T cells from normal individuals. Rheumatology 2004, 42:637644.

Acknowledgement Research was funded by Immune Regulation Ltd (GSP, VMC and MBS are shareholders in the company).

\section{P56}

High-dose chemotherapy and syngeneic stem cell transplantation in a patient with refractory rheumatoid arthritis: poor response associated with persistence of synovial abnormalities

M van Oosterhout', RJ Verburg', EWN Levarht', D Moolenburgh'2, I Bajema 3 , RY Barge ${ }^{4}$, WE Fibbe 4 , JM van Laar'

${ }^{1}$ Department of Rheumatology, Leiden University Medical Center, The Netherlands; ${ }^{2}$ Department of Rheumatology, Medical Center Alkmaar, The Netherlands; ${ }^{3}$ Department of Pathology, Leiden University Medical Center, The Netherlands; ${ }^{4}$ Department of Hematology, Leiden University Medical Center, The Netherlands Arthritis Res Ther 2005, 7(Suppl 1):P56 (DOI 10.1186/ar1577)

Background High-dose chemotherapy (HDC) and stem cell transplantation (SCT) is an experimental treatment option for patients with refractory rheumatoid arthritis (RA). The re-infusion of stem cells with a possible intrisic cell defect may be the cause for disease relapse despite increased sensitivity to disease-modifying anti-rheumatic drugs (DMARDs). This can be avoided by a syngeneic transplantation with healthy stem cells from an identical sibling.

Objectives We treated a 44-year-old woman, who had seropositive, erosive RA, with HDC and syngeneic SCT from her healthy identical twin sister. The patient had undergone multiple joint operations and had become refractory to conventional DMARDs, etanercept and infliximab.

Methods Outcome measures were DAS 28, rheumatoid factor titer (IgM), need for DMARD therapy and synovial inflammation for which tissue was obtained at baseline, 2, 3 and 6 months through knee arthroscopy. Stem cells from the donor were mobilized with filgrastim and collected by leukapheresis on day -1 and 0 . HDC consisted of cyclophosphamide (total dose $13.4 \mathrm{~g}$ intravenously) combined with $1 \mathrm{mg} / \mathrm{kg}$ prednisone. On day 0 an unmanipulated graft containing $182 \times 10^{9}$ leukocytes, containing $769 \times 10^{6} \mathrm{CD}^{4} 4^{+}$cells, was administered.

Results Before conditioning, infliximab, methotrexate and prednisone were discontinued, which resulted in a disease flare (Fig. 1). The treatment was well tolerated and the patient was discharged on day 14 without joint complaints. After 1 month disease activity flared accompanied by an acute phase response. Despite maintenance therapy with $10 \mathrm{mg}$ prednisone and re-institution of

\section{Figure 1}
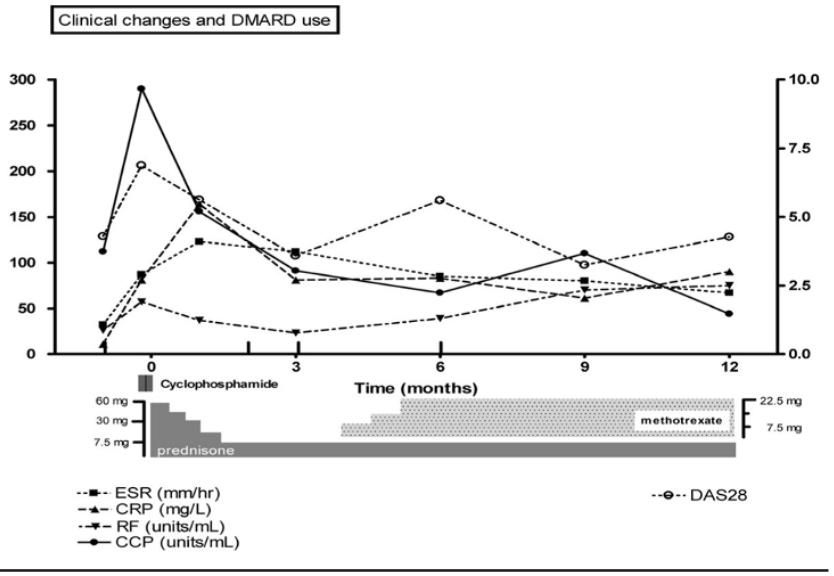

methotrexate up to $22.5 \mathrm{mg} /$ week she had continued active disease up to 1 year after SCT (Fig. 1). Synovial biopsies at all timepoints showed a marked infiltration with neutrophils and plasmacells, even 2 months after HDC. Immunohistological staining showed infiltration of activated $T$ cells $\left(C D 38^{+}\right)$, B cells $\left(C D 19^{+}\right)$and plasma cells $\left(\mathrm{CD} 138^{+}\right)$

Discussion HDC and syngeneic SCT did not result in remission of RA. The persistence of RF, anti-CCP (lgG) and plasma cells in the synovial tissue suggests host plasma cells were not eradicated by cyclophosphamide, nor replaced by donor plasma cells (rheumatoid factor and anti-CCP negative)

\section{P57}

Expression of IL-18 in muscle tissue of patients with treatment-resistant idiopathic inflammatory myopathies IE Lundberg1, S Barbasso', AK Ulfgren', JA Gracie'2, IB McInnes²

${ }^{1}$ Rheumatology Unit, Department of Medicine, Karolinska Institutet at Karolinska University Hospital, Solna, Stockholm, Sweden; ${ }^{2}$ Centre for Rheumatic Diseases, University of Glasgow, Glasgow, UK

Arthritis Res Ther 2005, 7(Suppl 1):P57 (DOI 10.1186/ar1578)

Background Idiopathic inflammatory myopathies, myositis, are characterized by infiltrates of $\mathrm{T}$ lymphocytes and macrophages in muscle tissue. Some patients with myositis are very resistant to immunosuppressive treatment. Thus new therapies are urgently required. An increased knowledge of the key molecular mechanisms in myositis would be important for development of new targeted therapies for these patients. IL-18 is a pleiotropic cytokine with proinflammatory and immunoregulatory effects. It has been shown to play an important pathogenic role in various autoimmune disorders (e.g. rheumatoid arthritis and Sjögrens syndrome). Whether IL-18 has a role in disease mechanism in myositis is not known. Objective To study the expression of IL-18 in muscle tissue of patients with idiopathic inflammatory myopathies.

Methods Thirteen treatment-resistant patients, six polymyositis, four dermatomyositis, one juveline dermatomyositis, and two inclusion body myositis with signs of active muscle inflammation, were included in our study. Muscle biopsies were investigated by immunhistochemistry using monoclonal antibodies against IL-18. Results Intracellular IL-18 expression was detected in all patients with polymyositis, dermatomyositis and inclusion body myositis. The IL-18 expression was predominantly localized to inflammatory cells, in large infiltrates and scattered in tissue, in endothelial cells of capillaries (11/13), in fibroblast-like cells and smooth muscle cells.

Conclusion This is the first study to demonstrate IL-18 expression at the protein level in muscle tissue from patients with idiopathic inflammatory myopathies. This was a consistent finding in mononuclear inflammatory cells in all three subsets of patients with treatment-resistant disease. These results suggest that IL-18 could have a role in the immunopathogenesis of myositis.

\section{P58}

Evaluation of chondrocyte micromass culture for the study of cartilage degradation TG Morgan, X Xu, AD Rowan, TE Cawston

Musculoskeletal Research Group, School of Clinical Medical Sciences, University of Newcastle upon Tyne, UK

Arthritis Res Ther 2005, 7(Suppl 1):P58 (DOI 10.1186/ar1579)

Background An understanding of the mechanisms involved in cartilage degradation that occur in rheumatoid arthritis and osteoarthritis is essential for the development of treatments to block disease progression. Culturing chondrocytes to synthesize a cartilage matrix would ideally provide a cartilage matrix of consistent composition that would reproducibly respond to different stimuli. When cultured in monolayers, chondrocytes convert to a fibroblastic morphology and gradually lose their native phenotype, no longer able to synthesize type II collagen and aggrecan. However, when chondrocytes are cultured at a high density in the presence of growth factors, the chondrocytes regain their native phenotype and native round morphology [1]

Objective To optimise the culture conditions for chondrocyte micromass synthesis of matrix components, and to evaluate the response of the culture to cytokine stimulation by measuring the degradation of matrix components.

Methods Chondrocytes extracted from bovine nasal cartilage were passaged several times to produce substantial cell stocks. Harvested cells were resuspended at $2 \times 10^{7}$ cells $/ \mathrm{ml}$ and seeded in the centre of each well of a 48-well plate $(20 \mu \mathrm{l} /$ well). Cultures were maintained in a serum-free medium with or without ITS (insulin, transferrin and selenium), ascorbic acid, and transforming 
Figure 1

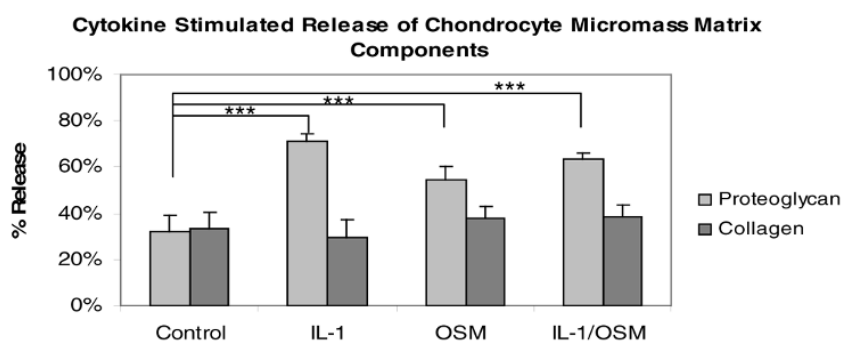

The effect on proteoglycan and collagen release when bovine nasal chondrocyte micromass cultured for 14 days in serum-free medium supplemented with $25 \mathrm{ng} / \mathrm{ml}$ transforming growth factor beta 1 and then stimulated for 14 days with IL-1 (1 ng/ml), oncostatin M (OSM) $(10 \mathrm{ng} / \mathrm{ml})$ and IL-1/OSM combination $(1 / 10 \mathrm{ng} / \mathrm{ml})$. A significant difference with control was identified by Student's $t$ test, ${ }^{* \star *} P<0.005$

growth factor beta 1 (TGF $\left.\beta_{1}\right)$ for 14-21 days. Histological analysis of the micromass cultures examined the presence of proteoglycans and type II collagen. The effects of cytokine stimulation with IL-1, oncostatin M (OSM), and the combination of both cytokines on matrix degradation were tested by analysing proteoglycan and collagen release. Collagenase activity was measured by bioassay. Results Both ITS and ascorbic acid were necessary for maximum collagen incorporation into micromass cultures. Collagen and proteoglycan incorporation increased with increasing TGF $\beta_{1}$ concentration and increasing culture duration, maximum incorporation detected when TGF $\beta_{1}$ was used at $30 \mathrm{ng} / \mathrm{ml}$ and cultures were maintained for 21 days. Histology showed that both proteoglycans and type II collagen were present throughout the chondrocyte micromass matrix. When micromass cultures were stimulated with IL-1, OSM and IL-1/OSM for 14 days, proteoglycan release was greater compared with control, but there was no difference for collagen release (see Fig. 1). Active collagenase levels were negligible, although high levels of pro-collagenase were detected.

Conclusion The chondrocyte micromass cultures synthesized a matrix that contained proteoglycan and type II collagen. The micromass culture may serve as a good model for studying proteoglycan but not collagen degradation.

Reference

1. Bradham DM, Horton WE: In vivo cartilage formation from growth factor modulated articular chondrocytes. Clin Orthopaed Relat Res 1998, 352:239-249.

Acknowledgment This work was funded by GlaxoSmithKline.

\section{P59}

A link between complement activation and anti-SSA in immune complex-induced cytokine production in systemic lupus erythematosus

\section{Mathsson, E Åhlin, H Storfors, J Rönnelid}

Unit of Clinical Immunology, Uppsala University, Uppsala, Sweden

Arthritis Res Ther 2005, 7(Suppl 1):P59 (DOI 10.1186/ar1580)

Background We have earlier shown that polyethylene glycol (PEG)-precipitated immune complexes (IC) from systemic lupus erythematosus (SLE) patients can induce IL-10 and IL-6 production from peripheral blood mononuclear cells. SLE is associated with IC-induced classical complement activation. Autoantibodies together with autoantigens released from apoptotic cells partly explain the IC formation. We therefore wanted to investigate how complement activation and levels of autoantibodies correlate to the amount of circulating $\mathrm{IC}$ and IC-induced cytokine production.

Others have previously shown an inverse correlation between IL-10 and IL-12 in SLE and we have shown that the degree of complement activation affects ICinduced IL-10 and IL-12 in different ways. A second aim was to investigate the effect of SLE IC on the production of IL-10 and IL-12.

Methods In total 195 SLE samples were investigated. Levels of autoantibodies (anti-nuclear antibodies, anti-DNA, SSA, SSB, RNP and Sm) were known for $116 / 195$ samples. In a first study we investigated 19 patients with paired serum samples obtained at different occasions and mostly showing activation of the classical complement pathway. Here we primarily wanted to evaluate the effect of disease activity on IC-induced cytokine production. In a second study 78 status were studied. Samples were PEG-precipitated and 10\% PEG precipitates were added to serum-free cultures of normal peripheral blood mononuclear cells, and the levels of IL-10, IL- 6 and IL-12p40 were measured in the supernatants after 20 hours. Classical complement pathway function, levels of C3, C3d and circulating IC were measured in all samples.

Results In paired analysis, increased complement activation (lowered classical function and raised $\mathrm{C} 3 \mathrm{~d} / \mathrm{C} 3$ ratios) was associated with increased IL-10 production $(P=0.046)$. For $\mathrm{IL}-6$ there was a parallel but non-significant trend. Decreased C3 levels were also associated with increased IL-12 production $(P=0.03)$. When investigating these complement-activating sera in crosssection we found increased IL-10 levels in $\mathrm{ENA}^{+}(P=0.03)$ and $\mathrm{DNA}^{+}$samples $(P=0.046)$ compared with $\mathrm{ENA}^{-}$and $\mathrm{DNA}^{-}$samples with the largest impact of anti-SSA/SSB $(P=0.02)$. The same pattern was found for IL-6.

In sera with normal complement levels there was no association between antibody status and IL-10 production, but IL-12 levels were increased in ENA ${ }^{+}$cultures $(P=0.01)$ and linked to anti-SSA/SSB $(P<0.0001)$. There was no association to anti-DNA.

Circulating IC in serum and PEG IC-induced IL-10 production correlated in samples from active SLE $(r=0.55, P=0.003)$, and we therefore used circulating IC as a surrogate marker for IC-induced IL-10 production in statistical evaluation of all sera with known antibody status. In analysis of variance with circulating IC as the dependent variable we found a strong interaction between decreased complement function and the occurrence of ENA, especially anti-SSA (complement $P<0.0001$, anti-SSA $P=0.0001$, interaction $P=0.002)$. Anti-DNA showed no association.

Conclusion We have found a link between classical complement activation, levels of circulating IC and induction of the cytokines IL-10, IL-6 and IL-12 with strong interaction between complement activation and presence of autoantibodies, notably anti-SSA. We hypothesize that, in quiescent SLE, autoantibodies circulate in monomeric form. In active SLE apoptotic cells release SSA forming IC with circulating anti-SSA, with subsequent induction of cytokines like IL-10.

PEG-precipitated SLE IC induce the production of IL-10 and IL-12 with parallel patterns depending on complement and autoantibodies. Inverse regulation of IL-10 and IL-12 in SLE therefore does not seem to depend on SLE-specific IC.

\section{P60}

The value of serum type II collagen epitope measurement in assessing early clinical response to treatment with antitumour necrosis factor alpha therapy in rheumatoid arthritis RH Mullan', T Lobanok2 ${ }^{2}$ C Walsh', L King ${ }^{3}$, O FitzGerald', B Bresnihan', U Fearon', R Poole ${ }^{2}$, DJ Veale ${ }^{1}$

${ }^{1}$ University College Dublin, Ireland; '2Shriners Hospital, Cedar Avenue, Montreal, PQ, Canada; ${ }^{3} /$ bex Pharmaceuticals, Montreal, PQ, Canada

Arthritis Res Ther 2005, 7(Suppl 1):P60 (DOI 10.1186/ar1581)

Introduction Current guidelines on the use of anti-tumour necrosis factor alpha $($ anti-TNF- $\alpha$ ) therapies in rheumatoid arthritis (RA) recommend a 3-month trial of the drug before a clinical assessment of treatment efficacy can be made. The serum level of the type II collagen propeptide CPII correlates with type II collagen synthesis, and is elevated in RA. The collagen cleavage neo-epitope C2C is specific for the destruction of type II collagen by the collagenases MMP-1, MMP-8 and MMP-13. Previously we have shown that the ratio of C2C/CPII is increased in osteoarthritis and correlates with cartilage destruction. The current pilot study assessed the utility of serum measurement of C2C and CPII in predicting early response to treatment with anti-TNF- $\alpha$ therapy in a group of 20 RA patients.

Method Twenty patients were assessed before commencement of either infliximab $(n=5)$ or etanercept $(n=15)$ therapy, and at 1-month and 3-month timepoints after therapy was commenced. Measurements of 28 swollen and tender joint counts for DAS28 score, erythrocyte sedimentation rate (ESR) and C-reactive protein (CRP) were taken at the time of each assessment. Additional serum was collected for measurement of CPII and C2C by ELISA. The change in serum levels of ESR, CRP, C2C, CPII and a ratio of C2C/CPII between 0 and 1 month was calculated, and then correlated with clinical outcomes at 3 months to assess their predictive value, using the Spearman Rank Correlation Coefficient.

Results The median DAS28 score fell from 6.20 to 3.74 following 3 months of treatment $(P=0.0004$, Wilcoxon Sign Rank Test). The change in $\mathrm{C} 2 \mathrm{C} / \mathrm{CPII}$ at 1 month of therapy was more closely associated with the change in swollen joint count at 3 months $(P=0.16)$ than either $\mathrm{CRP}(P=0.62)$ or ESR $(P=0.24)$. The change in $\mathrm{C} 2 \mathrm{C} / \mathrm{CPII}$ at 1 month was more closely associated with tender joint count at 3 months $(P=0.19)$ than either the $\operatorname{ESR}(P=0.59)$ or $\operatorname{CRP}(P=0.52)$. One-month changes in $\mathrm{C} 2 \mathrm{C}$ and $\mathrm{CPII}$ when used individually were not predictive of swollen joint count at 3 months $(P=0.55$ and $P=0.74$, respectively) or tender joint count at 3 months $(P=0.4$ and $P=0.8$, respectively).

Conclusion The ratio of $\mathrm{C} 2 \mathrm{C} / \mathrm{CPII}$ appears to offer a better method of predicting early response to biological therapy than the standard acute phase markers CRP 
and ESR. Measurement of the $\mathrm{C} 2 \mathrm{C} / \mathrm{CPII}$ ratio may more closely reflect cartilage changes in RA through measurement of both the synthesis and destruction of type II collagen. A further trial with larger patient numbers is required to fully assess the utility of this measure in predicting outcome to therapeutic intervention in the treatment of RA.

\section{P61}

\section{Oncostatin M in rheumatoid arthritis: a key cytokine acts synergistically with other proinflammatory cytokines to promote human cartilage loss} U Fearon', R Mullan', R Poole'2, T Markham 1', O FitzGerald', B Bresnihan', DJ Veale ${ }^{1}$ ${ }^{1}$ St Vincents University Hospital \& The Conway Institute, Dublin, Ireland; ${ }^{2}$ Shriners Hospital and McGill University, Montreal, Canada

Arthritis Res Ther 2005, 7(Suppl 1):P61 (DOI 10.1186/ar1582)

Introduction Oncostatin M (OSM), an IL-6 type cytokine, is raised in the rheumatoid arthritis (RA) joint and correlates with markers of inflammation and cartilage destruction. This study explores the mechanistic role of OSM in cell adhesion, angiogenesis and matrix degradation.

Methods Microvascular endothelial cells (EC), primary RA synovial fibroblasts (SFC), synovial explant culture and RASFC/normal cartilage co-cultures were stimulated with OSM $(20 \mathrm{ng} / \mathrm{ml}) \pm \mathrm{IL}-1(5 \mathrm{ng} / \mathrm{ml})$. Cell proliferation, cell adhesion molecules expression, tubule formation, cell migration and matrix degradation were assessed by proliferation, flow cytometry, matrigel angiogenesis and ELISA assays. Results OSM stimulated EC/RASFC (basal $=0.9 / 0.56$ to $1.56 / 1.94 ; P<0.05$, day 4) alone but demonstrated a synergistic effect in the presence of IL-1 $(P<0.01)$. OSM and IL-1 increased intercellular adhesion molecule expression, with no effect on vascular cell adhesion molecule or platelet endothelial cell adhesion molecule. OSM had an additive effect with IL-1 on intercellular adhesion molecule expression $(P<0.05)$. OSM increased $\mathrm{EC}$ tubule formation (basal $=$ $20 \pm 1.5$ to $47 \pm 3.7$; mean \pm standard error, $P<0.01$ ), and increased EC migration (basal $=1.265 \pm 0.115$ to $1.67 \pm 0.187 ; P<0.05$ ). OSM synergistically increased TIMP-1 production in EC, SFC and synovial explants in the presence of $\mathrm{IL}-1$, with no effect on $\alpha v \beta 3$ or urokinase (-type plasminogen activator) receptor expression. OSM and IL-1 stimulated MMP-1 and MMP-13 expression in cartilage and SFC alone $(P<0.05)$ but had a significant threefold synergistic induction when cultured together $(P<0.01)$. Cartilage sections stained with safranin-O demonstrated almost complete depletion of proteoglycan expression in RASFC/cartilage co-cultures following 4 weeks incubation with IL-1/OSM. This was reflected in the supernatants where IL-1 and OSM together synergistically increased GAG release from a basal level of 3.5-9.1 ( $\mu \mathrm{g} \mathrm{GAG/mg}$ cartilage weight) compared with either IL-1 or OSM alone.

Conclusion OSM plays a critical role in RASFC activation and cartilage invasion, also promoting angiogenesis. Its ability to act synergistically with other proinflammatory cytokines such as IL-1 further supports a key role for OSM in RA.

\section{P62}

Methotrexate, through adenosine release, downregulates tumor necrosis factor alpha-induced synovial fibroblast

\section{IL-15 expression and proliferation}

\section{E Miranda, M Benito, A Balsa, E Martin-Mola}

Department of Rheumatology, Hospital La Paz, Madrid, Spain

Arthritis Res Ther 2005, 7(Suppl 1):P62 (DOI 10.1186/ar1583)

Background Tumor necrosis factor alpha (TNF- $\alpha$ ) is found at high concentrations in the rheumatoid joint and induces upregulation of synovial fibroblast IL-15, a cytokine known to induce fibroblast proliferation through an autocrine loop. The mechanism of action of low-dose oral methotrexate (MTX) is not well understood. By inhibiting AICAR transformylase, MTX has been described to induce the extracellular release of the potent anti-inflammatory autacoid adenosine.

Objective To test the effect of MTX on TNF- $\alpha$-induced synovial fibroblast IL-15 expression and proliferation.

Methods Synovial fibroblasts (Sfib) isolated from surgical specimens of rheumatoid arthritis (RA) patients $(n=10)$ were cultured in six-well plates. Sfib were stimulated with $10 \mathrm{ng} / \mathrm{ml}$ TNF- $\alpha$ and pretreated for 2 hours with medium or MTX at varying doses $(0,0.01,0.1,1,10 \mu \mathrm{M})$ in the presence or absence of adenosine deaminase, DPCPX (adenosine A1 receptor antagonist) or DMPX (adenosine A2 receptor antagonist). Surface and intracellular IL-15 protein were determined by immunofluorescence and flow cytometry. IL-15 mRNA was measured by real-time quantitative RT-PCR in a LightCycler instrument (Roche). Sfib proliferation was evaluated using the alamar blue bioassay.
Results TNF- $\alpha$ induced an upregulation of intracellular and surface IL-15 protein expression and of IL-15 mRNA, together with an increased Sfib proliferation rate. Sfib proliferation was significantly inhibited by a neutralizing anti-IL15 monoclonal antibody but not by an isotype control antibody. In the presence of MTX, TNF- $\alpha$ induced IL-15 upregulation and Sfib proliferation were reduced by $40-65 \%$ in a dose-dependent manner. Adenosine deaminase reversed the effects of MTX as did addition of DMPX but not DPCPX 1 hour prior to MTX.

Conclusion MTX interferes with TNF- $\alpha$-induced synovial fibroblast IL-15 upregulation and proliferation. This effect seems to be mediated through adenosine release and adenosine $\mathrm{A} 2$ receptor engagement. This may be a mechanism by which MTX controls the aggressive behavior of synovial fibroblasts in RA.

Acknowledgements This work was supported by Ministerio de Educación y Ciencia Programa Ramón y Cajal (EM) and Ministerio de Educación y Ciencia grant SAF 2003-01670 (EM and MB).

\section{P63}

Influence of HLA-DR genes on the production of rheumatoid arthritis-specific autoantibodies to citrullinated fibrin I Auger', M Sebag'2, C Vincent'2, S Guis', L Nogueira², B Svensson ${ }^{3}$, A Cantagrel ${ }^{4}$, G Serre ${ }^{2}$, J Roudier ${ }^{1}$

1 INSERM UMR 639, Medical School and Rheumatology Ward, La Conception Hospital, Marseille, France; ${ }^{2}$ CNRS-Toulouse III University UMR 5165, Purpan

Hospital, Toulouse, France; ${ }^{3}$ Rheumatology Ward, Helsingborg Lasarett AB, Helsingborg, Sweden; ${ }^{4}$ Toulouse III University EA 2405 and Rheumatology Ward, Rangueil Hospital, Toulouse, France

Arthritis Res Ther 2005, 7(Suppl 1):P63 (DOI 10.1186/ar1584)

Rheumatoid arthritis (RA), a chronic inflammatory joint disease, develops in patients expressing particular HLA-DR alleles. RA patients' sera contain antibodies to a post-translationally modified form of fibrin on which arginyl residues are transformed in citrullins.

We tested whether HLA-DR alleles influence the production of anti-citrullinated fibrin antibodies in RA patient sera and whether the replacement of arginyl residues by citrullyl residues on fibrin peptides could modify their binding to RA-associated HLA-DR molecules and their recognition by T cells in RA patients and controls.

We found that RA-associated HLA-DR alleles are also associated with presence of anti-citrullinated fibrin antibodies in RA patient sera. Multiple peptides from the alpha and beta chain of fibrin are capable to bind many HLA-DR alleles. RA-associated HLA-DR alleles are good fibrin peptide binders. However, citrullination does not influence fibrin peptide binding to HLA-DR or fibrin peptide recognition by T cells.

Finally, peripheral blood $T$ cells that recognize native or citrullinated fibrin peptides are common in RA patients and very uncommon in normal controls. These results suggest that citrullination of fibrin has nothing to do with peptide/ HLA-DR/T cell interaction and is merely involved in the definition of $B$-cell epitopes.

\section{P64}

Bone marrow as a secondary lymphoid organ: mature T-cell subsets in bone marrow from rheumatoid arthritis and osteoarthritis patients

E Warnawin', T Burakowski', L Jung'2, P Maldyk'2, W Maslinski'

${ }^{1}$ Department of Pathophysiology and Immunology, Institute of Rheumatology, Warsaw, Poland; ${ }^{2}$ Clinic of Orthopedic Surgery, Institute of Rheumatology, Warsaw, Poland

Arthritis Res Ther 2005, 7(Suppl 1):P64 (DOI 10.1186/ar1585)

Introduction Recent data indicate that bone marrow plays an important role not only as a primary lymphoid organ responsible for haemopoiesis, but also as a secondary lymphoid organ with capability of antigen presentation exceeding that of lymph nodes. Although in chronic inflammatory/immune disease, like rheumatoid arthritis (RA), bone marrow participates in the initiation and/or perpetuation of the disease, there is little information about the real number of lymphocyte subpopulations in the bone marrow of these patients and how they can be modulated by T-cell growth factors. IL-15 acting through IL-15 receptors (including high-affinity IL-15R alpha chain) is a key cytokine influencing the development of natural killer cells in bone marrow, and proliferation and maintenance of the memory $\mathrm{CD}^{+}$cell pool. However, there is no information about the levels of IL-15 in bone marrow. Objectives To compare the real cell numbers within lymphocyte subsets in bone marrow isolated from RA and osteoarthritis (OA) patients. To measure the levels of soluble IL-15 and surface-expressed IL-15R alpha in bone marrow plasma and cells, respectively. To analyze the presence of memory and actively proliferating T cells in the bone marrow. 
Methods Bone marrow samples, obtained from RA and OA patients (mean age $53.1 \pm 10.6$ years and $54.3 \pm 13.6$ years, respectively) undergoing joint replacement surgery, were diluted four times in heparinized PBS. Bone marrow plasma samples were obtained by centrifugation and levels of IL-15 were measured using specific ELISA. The real number of lymphocytes stained for $\mathrm{CD}^{+}$and CD19+ were counted in the presence of TruCount beads using flow cytometry. Surface-expressed IL-15R was done on cells separated by gradient centrifugation, acid wash of surface-bound IL-15 and flow cytometric analysis. Surface expression of CD45RO was evaluated by flow cytometry. Cell proliferation was measured by intracellular expression of Ki-67.

Results There were twice as many $\mathrm{T}\left(\mathrm{CD}^{+}\right)$cells in RA in comparison with $\mathrm{OA}$ bone marrow $\left(6.1 \pm 2.8\right.$ versus $3.2 \pm 1.6$ cells $\times 10^{6} / \mathrm{ml}$ bone marrow, $P=0.008)$. In contrast, only $42 \%$ of $B\left(C D 19^{+}\right)$cells present in OA were present in RA bone marrow $\left(2.0 \pm 0.9\right.$ versus $0.85 \pm 0.3$ cells $\times 10^{6} / \mathrm{ml}$ bone marrow, $P=0.02$ ). Interestingly, both $\mathrm{CD} 3^{+} \mathrm{CD} 4^{+}$and $\mathrm{CD} 3^{+} \mathrm{CD} 8^{+}$cells obtained from $\mathrm{RA}$ patients expressed a significantly higher level of CD45RO $(P=0.002$ and $P=0.001$, respectively), measured as the mean fluorescence intensity, than in OA. In addition, there was a tendency (although not statistically significant, $P=0.08$ ) for a higher percentage of $\mathrm{CD}^{+} \mathrm{CD} 8^{+} \mathrm{CD} 45 \mathrm{RO}^{+}$cells in bone marrow from RA patients in comparison with OA patients (38.7\% versus $28.1 \%)$. Interestingly, lymphocytes isolated from RA patients expressed a significantly higher level of surface IL-15R alpha chain $(P=0.01)$, indicating their activation status. Cells isolated from RA bone marrow bound significantly more Ki-67 than cells isolated from OA bone marrow, indicating that RA cells proliferated more vigorously There were elevated levels of IL-15 in bone marrow plasma from RA in comparison with OA patients $(1304.5 \pm 956.3 \mathrm{pg} / \mathrm{ml}$ and $760 \pm 238.7 \mathrm{pg} / \mathrm{ml}$, respectively, $P=0.01$ )

Conclusion Lymphocytes obtained from RA patients expressed higher levels of IL-15R alpha and proliferated more rapidly than lymphocytes obtained from OA patients. It is likely that locally overproduced IL-15 is responsible for the elevated number of $\mathrm{T}$ cells in RA bone marrow. Higher density of $\mathrm{CD}_{4} \mathrm{RO} \mathrm{O}^{+}$memory marker expressed on T cells from RA patients further supports the role of IL-15 as a known growth factor for memory $\mathrm{T}$ cells. A significantly lower B-cell number in RA than in OA suggests that these cells actively emigrate from RA bone marrow to peripheral blood and affected joints. Distinct subpopulations of mature lymphocytes present in bone marrow from RA and OA patients indicate that the bone marrow acts as a secondary lymphoid organ that actively contributes to the pathogenesis of RA.

\section{P65}

Signaling pathways involved in TRAIL-induced rheumatoid arthritis synovial fibroblast proliferation J Morel'1,2, R Audo ${ }^{2}$, B Combe ${ }^{1,2}$

${ }^{1} \mathrm{CHU}$ Lapeyronie, Montpellier, France; ${ }^{2}$ INSERM U454, Montpellier, France Arthritis Res Ther 2005, 7(Suppl 1):P65 (DOI 10.1186/ar1586)

Tumor necrosis factor alpha-related apoptosis inducing ligand (TRAIL) is a proapoptotic factor that can also induce cell proliferation. The role of TRAIL in rheumatoid arthritis (RA) is still unclear. Previously, we reported that TRAIL induces RA fibroblast-like synoviocyte (FLS) proliferation. We now investigate the intracellular mechanisms involved in TRAIL-induced cell proliferation. Therefore, we tested the effect of TRAIL on signaling pathways including MAP kinases (p38 and ERK1/2) and PI3 kinase/Akt, known to control cell proliferation. For all these experiments, the concentration of TRAIL used to stimulate cell was $0.5 \mathrm{nM}$. TRAIL induced p38 MAP kinase but with a lower intensity in comparison with tumor necrosis factor alpha (TNF- $\alpha)(n=3)$. TRAIL also induced ERK1/2 and Akt phosphorylation in RA FLS in a time-dependent manner, showing maximum activation between 5 and 10 min by western blot $(n=3)$. This kinetic and intensity of ERK and Akt activation were similar to positive control TNF- $\alpha(n=3)$. The transcription factor NF-KB plays a major role in cell survival and is activated by MAP kinases and Akt. When NF- $\mathrm{BB}$ is activated, it translocates from the cytoplasm to the nucleus. We examined NF- $\mathrm{KB}$ translocation into the nucleus of RA FLS following TRAIL stimulation using immunofluorescence. RA FLS treated with TRAIL did not induce a nuclear uptake of NF- $\mathrm{KB}(n=3)$. The percentages of RA FLSpositive NF- $\kappa B$ translocation was increased from $4.3 \pm 0.9 \%$ to $8.9 \pm 2.3 \%$ when stimulated with TRAIL. For the positive control IL-1 $\beta$, this percentage increased to $97.9 \pm 1.1 \%$. This result was confirmed by western blot. To determine the implication of MAP kinases, and PI3 kinase/Akt in TRAlL-induced proliferation, we therefore tested different specific signaling inhibitors including $\mathrm{PI} 3$ kinase inhibitor LY294002, ERK1/2 inhibitor PD98059, and p38 inhibitor SB203580. RA FLS were pretreated with the specific inhibitors at different concentrations or $\mathrm{Me}_{2} \mathrm{SO}$ vehicle control for 1 hour and were then stimulated with TRAIL. Proliferation was assessed according to the level of incorporated tritiated thymidine. ERK
TRAIL-induced proliferation in a dose-dependent manner $(n=3)$. PI3 kinase inhibitor LY294002 almost completely blocked proliferation promoted by TRAIL. These results highlight the main role of Akt in TRAIL-mediated proliferation.

\section{P66}

Local IL-18 gene transfer prevents severe joint destruction in murine collagen-induced arthritis by induction of IL- 4 and osteoprotegerin

\section{LAB Joosten, RL Smeets, MI Koenders, MMA Helsen,}

B Oppers-Walgreen, FAJ van de Loo, E Lubberts, WB van den Berg

Rheumatology Research and Advanced Therapeutics, Department of

Rheumatology, University Medical Center Nijmegen, The Netherlands

Arthritis Res Ther 2005, 7(Suppl 1):P66 (DOI 10.1186/ar1587)

Introduction IL-18 is a member of the IL-1 family of proteins that exerts proinflammatory effects and is a pivotal cytokine for the development of T-cell-mediated immune responses. IL-18 can promote both the Th1 and Th2 pathways. Recent studies indicated IL-18 as an important stimulator of chronic inflammation in human diseases such as rheumatoid arthritis, Crohn's disease and several allergic disorders.

Objective To investigate the aggravating effect of local IL-18 overexpression on both joint inflammation and joint destruction in murine collagen-induced arthritis. Methods: Collagen-induced arthritis was induced in DBA-1 mice by intradermal injection of $100 \mu \mathrm{g}$ bovine type II collagen in FCA. At day 21 the mice were boosted with $100 \mu \mathrm{g}$ type II collagen. IL-18 gene transfer was performed on day 22 by intra-articular injection of $1 \times 10^{7} \mathrm{pfu}$ AdmlL-18. As control we injected $1 \times 10^{7}$ pfu Ad5del70.3 Histopathology was examined at days 5 and 12 after AdIL-18 injection. Bone destruction was investigated using $\mathrm{X}$-ray analysis. In addition, both systemic and local cytokine levels were determined in sera and patellae washouts, using a multiplex bead array (Luminex Technology). Osteoprotegerin levels were examined by ELISA.

Results Here we report that overexpression of IL-18 in knee joints of collagen type II primed DBA-1 mice resulted in aggravation of joint inflammation. Enhanced influx of proinflammatory cells, predominantly polymorphonuclear cells, was seen already at day 5 in both synovial tissue and the joint cavity. At day 12 extremely severe joint inflammation was seen in the AdlL-18 injected knee joint, whereas the Ad5del70.3-injected or saline-injected mice showed modest joint inflammation. Of high interest, although severe joint inflammation developed after IL-18 gene transfer, no signs of joint destruction were noted at day 12 after IL-18 overexpression. Histopathological and $\mathrm{X}$-ray analysis revealed that both cartilage and bone destruction were completely prevented after intra-articular IL-18 exposure during collagen-induced arthritis. In addition, we found that local overexpression of IL-18 resulted in elevated levels for IL-4 in both serum (29 \pm 14 versus $4 \pm 3 \mathrm{pg} / \mathrm{ml}$ ) and in synovial tissue washouts $(1240 \pm 220$ versus $24 \pm 7 \mathrm{pg} / \mathrm{ml})$ when compared with Ad5del70.3. Remarkably high levels of osteoprotegerin were found in synovial tissue washouts at day 12 after IL-18 gene transfer compared with Ad5del70.3 vector (1570 \pm 270 versus $210 \pm 20 \mathrm{pg} / \mathrm{ml}$ ). Moreover, local IL-10 levels were strongly enhanced whereas IL-1 $\beta$ levels were significantly reduced after IL-18 gene transfer.

Conclusion The present study clearly demonstrated that local IL-18 overexpression accelerates joint inflammation, but prevents development of severe cartilage and bone destruction. High levels of both IL-4 and osteoprotegerin indicate establishment of a non-destructive type 2 inflammation. These data suggest that IL-18 may have a dual role in chronic destructive arthritis and that therapies based on local IL-18 blockade might be inadequate in rheumatoid arthritis.

\section{P67}

Gene expression profiling provides a link between high inflammatory synovitis and myofibroblast-like synoviocytes TCG Timmer', PV Kasperkovitz', LGM van Baarsen'1, P-P Tak'2, TWJ Huizinga ${ }^{3}$, TCTM van der Pouw Kraan', CL Verweij'

${ }^{1}$ Department of Molecular Cell Biology and Immunology, VUMC, Amsterdam, The Netherlands; ${ }^{2}$ Division of Clinical Immunology and Rheumatology, AMC Amsterdam, The Netherlands; ${ }^{3}$ Department of Rheumatology, LUMC, Leiden, The Netherlands

Arthritis Res Ther 2005, 7(Suppl 1):P67 (DOI 10.1186/ar1588)

Objective The molecular pathogenesis of rheumatoid arthritis (RA) is still poorly understood. Given the heterogeneity in gene expression patterns and cellular distribution between RA synovial tissue, we determined whether this variability is also reflected at the level of fibroblast-like synoviocytes (FLS) cultured from those synovial tissues.

Methods Gene expression profiles in FLS from 19 RA synovial tissues were analyzed using cDNA microarrays and hierarchical cluster analysis. To validate the subclassification, we performed prediction analysis and principal component 
analysis. Genes that differed significantly in expression between FLS cultures were selected using statistical analysis of microarrays. Real-time PCR was performed to validate microarray data. Immunostaining was applied to study the expression of the genes of interest in FLS and synovial tissues.

Results Hierarchical clustering identified two main groups of FLS characterized by distinctive gene expression profiles. FLS from high inflammatory synovial tissues revealed increased expression of a transforming growth factor beta/ Activin A inducible gene program that is characteristic of myofibroblasts, a cell type that is considered to be involved in wound healing, whereas increased growth factor (IGF2/IGFBP5) production appears to constitute a characteristic feature of FLS derived of low inflammatory synovial tissues. The molecular feature that defines the myofibroblast-like phenotype is reflected as an

increased proportion of myofibroblast-like cells in the heterogeneous FLS population. Myofibroblast-like cells are also found upon immunohistochemical analysis of synovial tissue.

Conclusion The data support the notion that heterogeneity between synovial tissues is reflected in FLS as a stable trait, and provide evidence for a possible link between FLS behavior and the inflammatory status of RA synovium.

\section{P68}

Anakinra (Kineret) in psoriasis and psoriatic arthritis: a single-center, open-label, pilot study A Gibbs, T Markham, C Walsh, B Bresnihan, D Veale, O FitzGerald

St Vincents University Hospital, Elm Park, Dublin, Ireland Arthritis Res Ther 2005, 7(Suppl 1):P68 (DOI 10.1186/ar1589)

Backround Both gene and protein expression of IL-1 are highly upregulated in skin and synovium of patients with psoriatic arthritis (PsA) and psoriasis. IL-1 is known to play a key role in keratinocyte activation, matrix metalloproteinase upregulation, differentiation of osteoblast precursors into mature osteoblasts, angiogenesis and the development of erosive disease. Thus, the purpose of the study is to assess the effects of IL-1 inhibition on the clinical features of psoriasis and PsA disease activity, on synovial and cutaneous immunohistology and on magnetic resonance imaging (MRI) features of an affected knee joint.

Methods To date, eight PsA patients (six male and two female, average age 41 years [22-61 years]) with active skin and joint disease have been enrolled for treatment over 12 weeks with $100 \mathrm{mg}$ anakinra daily by subcutaneous injection. Physical examination, patient and physician global assessment of disease activity (on a scale of $0-3$ ), tender and swollen joint scores, erythrocyte sedimentation rate, C-reactive protein and PASI scores were performed at all visits. Plain X-rays of hands, wrists, knees and feet were performed at baseline. A biopsy of a psoriatic plaque, multiple arthroscopic synovial biopsies and a MRI scan of an inflamed knee joint were performed at baseline and week 12. HAQ scores, patient assessment of disease activity and pain using visual analogue scales were obtained at weeks 0 and 12. PsA-OoL were calculated at weeks 0 and 12. The primary endpoint was the proportion of patients who met the Psoriatic Arthritis Response Criteria (PsARC) at 12 weeks.

Results Seven patients completed the study. One male patient was non-compliant with the study protocol and susbsequently withdrew before completion of the study. Baseline physical examination revealed psoriasis and active synovitis in all patients. Three of the seven patients $(43 \%)$ met the PsARC at week 12. Mean HAQ scores improved from $1.23(0.75-1.9)$ to $0.96(0.25-1.6)$, while mean PsAQoL scores changed from 22/46 (9-41) to 20/46 (5-40). Other relevant data are presented in Table 1.

Conclusion This ongoing study has shown anakinra to be of modest benefit in patients with PsA. Further patient recruitment, MRI assessment and immunohistochemical analysis of tissue samples is ongoing.
P69

Engagement of the p55 TNF-R for different periods of time induces qualitatively distinct pathways of tumour necrosis factor signalling and gene expression profiles in murine $\mathrm{T}$ cells P Vagenas', JM Clark', J Testar', M Panesar', I Udalova', T Freeman², P Lyons $^{2}$, AP Cope ${ }^{1}$

${ }^{1}$ The Kennedy Institute of Rheumatology, Faculty of Medicine, Imperial College London, UK; ${ }^{2}$ MRC Human Genome Resource Centre, Hinxton, Cambridge, UK Arthritis Res Ther 2005, 7(Suppl 1):P69 (DOI 10.1186/ar1590)

To provide insight into the mechanisms whereby sustained tumour necrosis factor (TNF) stimulation promotes chronic inflammatory responses, we have examined TNF-R signalling pathways in murine T-cell hybridomas. Prolonged TNF signalling attenuates p55 TNF-R (TNF-RI)-induced JNK activation through mechanisms that are NF- $\mathrm{KB}$ dependent and that target receptor proximal pathways, since arsenite-induced JNK activation is preserved. In contrast, phosphorylation and degradation of $\mathrm{lkB}$ alpha, combined with nuclear translocation and gel shift assays, demonstrate that prolonged TNF stimulation promotes chronic NF- $\kappa B$ activation. However, acute and chronic TNF signals are qualitatively distinct. In chronic TNF-treated T cells degradation of $\mathrm{lkB}$ alpha and nuclear translocation of RelA (p65) are not as pronounced as that in cells following acute stimulation, while translocation of NF- $\mathrm{KB} 1$ ( $\mathrm{p50}$ ) is enhanced. Gel shift assays demonstrate consistent differences in the binding of Rel family proteins to DNA, since acute TNF stimulation induces formation of a dominant DNA binding complex comprising p65:p50 heterodimers (complex I), while the binding capacity of a second complex (complex II), which may include p50:p50 homodimers, is increased in nuclear extracts of chronic TNF-stimulated cells. Preliminary data indicate that chronic but not acute TNF stimulation also induces RelB nuclear translocation and DNA binding. A direct comparison of gene expression profiles in control, acute and chronic TNF-stimulated T cells suggests that differences in TNF-R signalling translate to distinct gene fingerprints. By interrogation of extended fragments of genomic sequence from 66 TNF-induced genes, we have mapped the DNA binding consensus sequence sites for different transcription factors. For the classical NF- $\kappa B$ DNA binding motif GGGRNNYYCC our analysis revealed multiple kB 'hits' in 55/66 TNF-induced genes. Variation in kB motifs was observed across gene clusters. Our results have uncovered differences between acute and chronic TNF signals that may arise through variation in signal input, specific requirements for Rel family dimers, divergence in $\mathrm{kB}$ consensus sequences and differences in affinities of protein-DNA binding.

Acknowledgements This work was supported by The Wellcome Trust and by arc.

\section{P70}

Expression of Toll-like receptor (TLR) 2, TLR3, TLR4 and TLR7 is increased in rheumatoid arthritis synovium and regulates cytokine production by dendritic cells upon stimulation of TLR specific pathways

MF Roelofs, LAB Joosten, S Abdollahi-Roodsaz, AWT van Lieshout, TRDJ Radstake, WB van den Berg

Department of Rheumatology, Rheumatology Research and Advanced

Therapeutics, University Medical Center Nijmegen, The Netherlands

Arthritis Res Ther 2005, 7(Suppl 1):P70 (DOI 10.1186/ar1591)

Background Rheumatoid arthritis (RA) is a chronic autoimmune disease that is characterized by inflammation of the synovial joints, which leads to joint destruction. Dendritic cells (DC) are specialized antigen presenting cells, which are activated by 'pathogen associated molecular patterns' via toll-like receptors (TLRs). TLR3 and TLR7 are important in the recognition of viral RNA, whereas TLR2 and TLR4 are involved in the recognition of bacterial components and host-derived molecules, including heat shock proteins, fibronectin, hyaluronic acid. TLR and $\mathrm{DC}$ are critical in the regulation of immune responses.

Aim To determine the expression of TLR2, TLR3, TLR4 and TLR7 in synovial biopsies and to evaluate the potential differences in DC maturation and cytokine production upon TLR-mediated triggering of cells from RA patients and healthy controls.

Table 1 (abstract P68)

\begin{tabular}{|c|c|c|c|c|c|c|c|c|c|}
\hline & $\begin{array}{l}\text { Patient } \\
\text { assessment } \\
\text { of disease } \\
\text { activity } \\
(0-3)\end{array}$ & $\begin{array}{l}\text { Physician } \\
\text { assessment } \\
\text { of disease } \\
\text { activity } \\
(0-3)\end{array}$ & $\begin{array}{c}\text { Tender } \\
\text { joint } \\
\text { score } \\
(0-78)\end{array}$ & $\begin{array}{c}\text { Swollen } \\
\text { joint } \\
\text { score } \\
(0-76)\end{array}$ & $\begin{array}{c}\text { Patient } \\
\text { assessment } \\
\text { of disease } \\
\text { activity } \\
\text { on VAS }\end{array}$ & $\begin{array}{c}\text { Patient } \\
\text { assessment } \\
\text { of pain } \\
\text { on VAS }\end{array}$ & $\begin{array}{l}\text { PASI } \\
\text { score }\end{array}$ & $\begin{array}{c}\text { Erythrocyte } \\
\text { sedimentation } \\
\text { rate }\end{array}$ & $\begin{array}{c}\text { C-reactive } \\
\text { protein }\end{array}$ \\
\hline Baseline & $2.14(1-3)$ & $2.28(1-3)$ & $24(11-38)$ & $20(7-29)$ & $61(27-85)$ & $61(21-90)$ & $5.21 \%(1-11)$ & $27(3-78)$ & $33(4-70)$ \\
\hline Week 12 & $1.7(1-3)$ & $1.7(1-3)$ & $16(12-21)$ & $11(5-25)$ & $43(12-70)$ & $46(20-71)$ & $4.5 \%(0-8)$ & $18(2-46)$ & $13(4-29)$ \\
\hline
\end{tabular}


Methods The presence of TLR2, TLR3, TLR4 and TLR7 in synovial tissue, from RA patients, osteoarthritis patients and healthy controls was studied with immunohistochemistry techniques. Furthermore, monocyte-derived dendritic cells from RA patients and healthy controls were cultured for 6 days and subsequently stimulated via specific TLR pathways; TLR2 ( pam $_{3} \mathrm{cys}$ ), TLR3 (poly(IC)), TLR4 (LPS) and TLR7 (R848). We determined the expression of cell surface markers (CD14, CD80, CD83, CD86, MHCl and MHCll) by flow cytometry and tumour necrosis factor alpha, IL-6, IL-10 and IL-12 production was measured by multiplex cytokine bead arrays.

Results TLR2, TLR3, TLR4 and TLR7 expression was markedly increased in synovial tissue from RA patients, compared with synovial tissue from osteoarthritis patients and healthy controls. After stimulation via specific TLR pathways, flow cytometry showed a clear DC maturation upon stimulation via all TLR pathways without any difference between RA cells and cells from healthy controls. However, cytokine production by DC from RA and controls was clearly different. RA DC produced increased levels of the proinflammatory cytokines tumour necrosis factor alpha and IL-6 upon stimulation of TLR2 or TLR4. In contrast, such differences were not noted after TLR3 and TLR7 stimulation. Triggering of TLR3 and TLR7 pathways resulted in increased production of IL-12, which was equal between RA and control DC. Intriguingly, simultaneous stimulation of TLR3/4, TLR3/7 and TLR4/7 pathways resulted in a marked synergy with respect to the production of proinflammatory mediators.

Conclusion These results point out that despite a comparable DC phenotype, RA cells produce higher cytokine levels upon stimulation of TLR2 and TLR4 compared with healthy controls. The combination of specific TLR stimulations resulted in a distinguished synergy in cytokine production. Here, we postulate the hypothesis that the persistence of viral material in synovial tissue triggers the TLR3 and TLR7 pathways, which subsequently results in inflammation and release of endogenous ligands, resulting in TLR4 stimulation that leads to a vicious circle of synovial inflammation.

\section{P71}

\section{Post-transcriptional regulation of proinflammatory cytokines in arthritis using gene delivery of AU-rich element binding factors}

M Khoury', F Apparailly', AR Clark ${ }^{3}$, P Plence', D Noel', C Jorgensen ${ }^{1,2}$

${ }^{1}$ INSERM U475, Université Montpellier I, Montpellier, France; ${ }^{2} \mathrm{CHU}$ Lapeyronie, service ImmunoRhumatologie, Montpellier, France; ${ }^{3}$ Kennedy Institute of Rheumatology Division, Faculty of Medicine, Imperial College London, UK Arthritis Res Ther 2005, 7(Suppl 1):P71 (DOI 10.1186/ar1592)

Background Monocytes and macrophages are abundant in rheumatoid synovial tissue and play a major role in the pathogenesis of rheumatoid arthritis (RA) by secreting proinflammatory cytokines such as tumour necrosis factor alpha (TNF- $\alpha$ ). An innovative approach is based on the regulation of mRNA stability and degradation, which constitute a critical step in the control of gene expression rather than neutralization of the downstream protein. Stability and degradation of TNF- $\alpha$ mRNA are regulated by cis-acting sequences as AU-rich elements (AREs). Tristetraprolin (TTP), a class of Cys-Cys-Cys-His $(\mathrm{CCCH})$ zinc finger proteins, was identified as the critical TNF- $\alpha$ ARE-binding protein. Importantly, TTP knockout mice develop inflammatory arthritis, dermatitis and myeloid hyperplasia, prevented by anti-TNF- $\alpha$ antibodies. A recent study suggested that a low TTP/TNF- $\alpha$ RNA expression ratio could indicate failure of RA patients to produce adequate amounts of TTP in response to increased TNF- $\alpha$ production. Our aim is to investigate the therapeutic potential of gene expression of ARE-binding elements for anti-TNF- $\alpha$ therapies. Methods We used the THP1 human monocyte cell line known for a high lipopolysaccharide (LPS)-induced TNF- $\alpha$ secretion. An expression vector containing TTP gene driven by the CMV promoter was constructed. TTP expression was evaluated by western blot following transfection by electroporation $(320 \mathrm{mV})$. The secretion of TNF- $\alpha$ by THP1 was assessed by ELISA before and after TTP transfection ( $1 \mu \mathrm{g} / 10^{6}$ cells), following LPS stimulation $(50 \mathrm{ng} / \mathrm{ml})$. Results A significant decrease in TNF- $\alpha$ expression was observed in the TTPtransfected THP1 cells, compared with a GFP mock control. This suppression increased over time and last at least 48 hours after LPS stimulation (23\% at 3 hours and $37 \%$ at 24 hours).

Conclusion These preliminary results support an important role of TTP in regulating TNF- $\alpha$ in monocytes and might be the new target for gene delivery in an antiTNF- $\alpha$ strategy in RA. In vivo evaluation of this strategy in experimental models of RA will be tested.
P72

Arthroscopic lavage with methylprednisolone is superior compared with either treatment alone in patients with inflammatory arthritis of the knee: a randomized prospective trial

M van Oosterhout', JK Sont ${ }^{2}$, I Bajema ${ }^{3}$, FC Breedveld1, JM van Laar1

${ }^{1}$ Department of Rheumatology, Leiden University Medical Center, The Netherlands; ${ }^{2}$ Department of Medical Decision Makingy, Leiden University Medical Center, The Netherlands; ${ }^{3}$ Department of Pathology, Leiden University Medical Center, The Netherlands

Arthritis Res Ther 2005, 7(Suppl 1):P72 (DOI 10.1186/ar1593)

Background Patients with recurring or persisting inflammatory arthritis of the knee despite anti-rheumatic therapies are frequently treated with intra-articular steroid injections. Lavage of a rheumatic joint may also be benificial by removing synovial debris and proinflammatory substances from the joint. Only uncontrolled studies have been performed so far and none of these has studied local synovial features. Objectives To compare the therapeutic effect of three local interventions: arthroscopic lavage with steroid application ( $A L+s t)$, arthroscopic lavage with placebo $(A L+p l)$ and needle biopsie with steroids $(N B+s t$, as a representative of joint injection). To study synovial tissue biopsies for predictive factors for the response to local therapy.

MethodsArthroscopic lavage with 1 I saline was performed under local anaesthesia using 2 trocharts. At the end of the procedure $80 \mathrm{mg}$ methylprednisolone or placebo was instilled. Needle biopsy was performed under local anaesthesia with 1 trochart and instillation of $80 \mathrm{mg}$ methylprednisolone. In all patients synovial tissue specimens were obtained and histologically analyzed. Kaplan-Meier curves were constructed depicting the time until recurrence of arthritis. Cumulative incidences of recurrence were used to calculate relative risks (RRs) between treatment groups.

Results Seventy-eight patients were equally randomized over the three interventions. $\mathrm{AL}+\mathrm{st}$ was the best therapy with a median time untill recurrence of 0.58 years, followed by $\mathrm{NB}+$ st ( 0.23 years) and $\mathrm{AL}+\mathrm{pl}(0.08$ years) (Fig. 1) The RR for recurrence compared with $\mathrm{AL}+\mathrm{st}$ was 2.0 for $\mathrm{NB}+\mathrm{st}(P=0.03)$ and 4.7 for $\mathrm{AL}+\mathrm{pl}(P<0.001)$. Synovial tissue analysis showed a relation between infiltrating lymphocytes and outcome of $\mathrm{AL}+\mathrm{st} ; \mathrm{RR}=2.7(P=0.01)$ in patients with less infiltrating lymphocytes. The opposite was found for fibrosis; $\mathrm{RR}=2.9(P=0.03)$ in patients with more fibrosis.

Figure 1

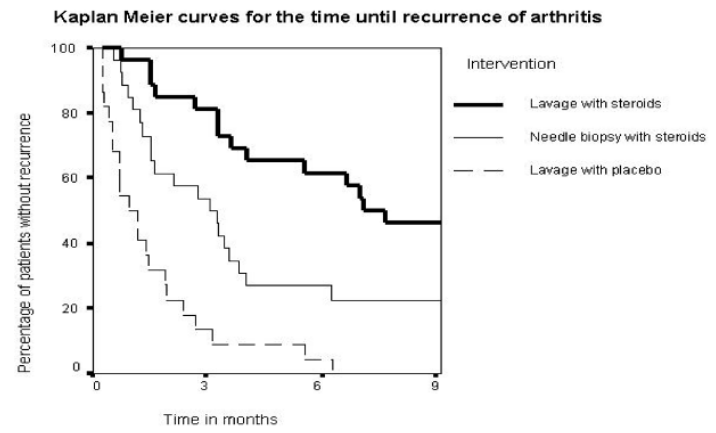

Kaplan-Meier curves for the time until recurrence of arthritis.

Conclusion Joint lavage preceding steroid injection had a marked effect on time until recurrence when compared with joint lavage or steroid injection alone. The clinical effect was correlated with the presence of a lymphocytic infiltrate and the absence of fibrosis.

P73

Phenotypic, genotypic and functional characterization of mesenchymal stem cells from synovial membrane compared with bone marrow

F Djouad1', D Noël', G Uzé², T Haüpl³, P Plence'1, C Bony¹, F Apparailly', C Jorgensen ${ }^{1,3}$

${ }^{1}$ INSERM U475, Montpellier, France; ${ }^{2}$ CNRS UMR 5124, University of Montpellier II, Montpellier, France; ${ }^{3}$ Charité Hospital, Berlin, Germany; ${ }^{4}$ Department of Immuno-rheumatology, Lapeyronie Hospital, Montpellier, France Arthritis Res Ther 2005, 7(Suppl 1):P73 (DOI 10.1186/ar1594)

Objective Mesenchymal stem cells (MSC) are progenitor cells of mesodermal lineages that are present in different tissues such as the fat pads and the bone 
marrow (BM) but also in the cartilage and the synovial membrane (SM). The aim of this study was to better characterize BM-derived and SM-derived MSC and discriminate between the two tissue sources.

Methods BM-MSC and SM-MSC were compared for the expression of cell surface markers and induction of MHC class I and class II molecules by interferon (IFN)- $\alpha 2$, IFN- $\beta$ and IFN- $\gamma$. Functional characterization was assessed through their differentiation potential towards chondrocytes and osteoblasts using quantitative RT-PCR, and their immunosuppressive properties by mixed lymphocyte reaction and indoleamine 2,3-dioxygenase activity. Using macroarray technology, the expression of 268 genes was monitored in four samples from BM-derived and SM-derived MSC.

Results BM-derived and SM-derived MSC were shown to express CD44, CD73, CD90 and CD105 in the same range and to respond to IFN stimulation. We showed that IFN- $\beta$, IFN- $\alpha 2$ and IFN- $\gamma$ are able to stimulate the expression of MHC class I and class II molecules on the MSC surface from both origins. Using quantitative analysis, we demonstrated similar differentiation capacities (osteogenesis and chondrogenesis induction) for BM-derived or SM-derived MSC. Moreover, our data are the first to show the same immunosuppressive features in mixed lymphocyte reaction ( $>90 \%$ of T-cell proliferation inhibition) and similar expression of indoleamine 2,3-dioxygenase in BM-derived and SM-derived MSC. Nevertheless, the macroarray analysis shows a statistically significant discrimination between SM-derived and BM-derived MSC, in particular inflammatory cytokine as IL6 and IL8 were upregulated in synoviocytes compared with BM-MSC. Validation in terms of expression of specific proteins is being undertaken.

Conclusion Altogether, the data indicate that BM-MSC and SM-MSC shared multiple phenotypic and functional properties. However, the genomic signature permits one to discriminate the MSC originating from the two different tissues.

\section{P74}

\section{Proinflammatory role for $\mathrm{AT}_{1}$ receptors in the rat synovium \\ AG Price', JC Lockhart', JS McLean', WR Ferrel/2}

${ }^{1}$ Biological Sciences, University of Paisley, UK; ${ }^{2}$ Centre for Rheumatic Diseases, Royal Infirmary, Glasgow, UK

Arthritis Res Ther 2005, 7(Suppl 1):P74 (DOI 10.1186/ar1595)

The increasing recognition that angiotensin II (Ang II) has proinflammatory roles suggests it may therefore contribute to chronic inflammatory conditions such as rheumatoid arthritis (RA), but this requires investigation. Walsh and colleagues demonstrated the presence and upregulation of $A T_{1}$ receptors [1] and angiotensin-converting enzyme [2] in the synovium of RA patients. The aim of this study was to elucidate the role of Ang II in rat knee joint pathophysiology and to investigate the anti-inflammatory potential of $\mathrm{AT}_{1}$ receptor antagonist losartan in models of arthritis. The second phase of this study was to determine the mechanism of any anti-inflammatory action of losartan. In vivo losartan has been reported to undergo hepatic first-pass metabolism [3], yielding two metabolites, EXP-3174 (the active metabolite) and EXP-3179. The latter is structurally similar to the nonselective cyclooxygenase inhibitor indomethacin. Since the anti-inflammatory benefit of cyclooxygenase inhibitors is well documented, we aimed to determine whether any anti-inflammatory benefit associated with losartan was attributable to $\mathrm{AT}_{1}$ receptor antagonism or inhibitory action on the prostanoid system.

In vivo experiments Experimental arthritis was induced under anaesthesia (halothane $/ \mathrm{O}_{2} / \mathrm{N}_{2} \mathrm{O}$ ) by intra-articular and peri-articular injection of Freund's complete adjuvant in adult male Wistar rats $(350 \pm 20 \mathrm{~g}$ body weight, mean \pm SEM, $n=6$ ). Knee joint swelling was measured using callipers and expressed as the percentage change from pre-induction values. The anti-inflammatory potential of losartan (Merck \& Co., USA) was tested both prophylactically and therapeutically. Prophylactically, losartan (15 mg/kg; subcutaneous) was given 1 hour prior to induction of inflammation and every 48 hours thereafter. Therapeutically, 12 days after the induction of inflammation, losartan $(15 \mathrm{mg} / \mathrm{kg}$; subcutaneous) treatment was started and dosing continued every 48 hours. The mechanism of action of losartan was investigated using EXP 3174 (Merck \& Co.), given prophylactically ( $15 \mathrm{mg} / \mathrm{kg}$; subcutaneous, maintenance every 48 hours) 1 hour prior to induction of inflammation (Freund's complete adjuvant).

In vitro experiments U937 human monocytes at a logarithmic phase of growth were harvested and adjusted to $10^{6} \mathrm{cell} / \mathrm{s} / \mathrm{ml}$ in the presence of $10 \mathrm{nM}$ phorbol 12myristate 13-acetate for 3 days to differentiate into adherent macrophages. Cells were then quiesced for 24 hours before experiments commenced. Cells were then stimulated with lipopolysaccharide $(50 \mathrm{ng} / \mathrm{ml})$ incubated in the presence of medium (control), Ang II $\left(10^{-9} \mathrm{~mol}\right)$ or losartan $\left(10^{-9} \mathrm{~mol}\right.$ to $\left.10^{-6} \mathrm{~mol}\right)$. Supernatants were collected and tumour necrosis factor alpha (TNF- $\alpha$ ) levels measured by ELISA

Prophylactic administration of losartan resulted in a significant reduction in knee joint swelling over 14 days (two-way analysis of variance [ANOVA], $P<0.00001$; $n=6$ ). Therapeutic losartan treatment reduced established knee joint swelling compared with vehicle control (two-way ANOVA, $P<0.00001 ; n=7$ ). Prophylactic administration of EXP-3174 also resulted in a reduced percentage increase in knee joint diameter, again found to be significant (two-way ANOVA, $P<0.00001 ; n=6-8)$. Losartan inhibited TNF- $\alpha$ production from U937 cells in a dose-dependent manner (cf. LPS-treated cells; $n=3$ ).

The anti-inflammatory benefit of both prophylactic and therapeutic losartan treatment suggests both an important role for Ang II in arthritis, and a novel therapeu- tic application for $\mathrm{AT}_{1}$ receptor antagonists. This anti-inflammatory action may be due to inhibition of TNF- $\alpha$ release from macrophages.

References

1. Walsh DA, Suzuki T, Knock GA, Blake DR, Polak JM, Wharton J: AT receptor characteristics of angiotensin analogue binding in human synovium. Br J Pharmacol 1994, 112:435-442.

2. Walsh DA, Mapp PI, Wharton J, Polak JM, Blake DR: Neuropeptide degrading enzymes in normal and inflamed human synovium. $A m$ Pathol 1993, 142:1610-1621.

3. Krämer C, Sunkomat J, Witte J, Luchtefeld M, Walden M, Schmidt B, Böger $\mathrm{RH}$, Forssmann W-G, Drexler H, Schieffer B: Angiotensin II receptorindependent antiinflammatory and antiaggregatory properties of losartan: role of the active metabolite EXP3179. Circ Res 2002, 90:770-776. Acknowledgements This study was funded by University of Paisley Research Funds and Merck \& Co, USA.

P75

\section{Proteomic characterisation of cell contact-dependent macrophage activation} MJ Peirce, R Wait, X Wu, S Begum, J Saklatvala, AP Cope

The Kennedy Institute of Rheumatology Division, Imperial College School of Medicine, London, UK

Arthritis Res Ther 2005, 7(Suppl 1):P75 (DOI 10.1186/ar1596)

The generation of tumour necrosis factor alpha (TNF- $\alpha$ ) in the rheumatoid synovium is widely perceived as pivotal to disease pathogenesis in rheumatoid arthritis (RA). While TNF- $\alpha$ production in dispersed synovial cell cultures is T-cell-dependent [1] there is little evidence of antigen-dependent T-cell activation. One explanation for this apparent paradox is the well-documented ability of appropriately activated $T$ cells to drive production of TNF- $\alpha$ from macrophages through a cell contact-dependent, antigen-independent mechanism [2]. Despite some promising leads (e.g. CD69 [3]), the identities of the relevant stimulating Tcell surface ligands, and the macrophage signalling pathways engaged following cell contact remain obscure. Our approach has been to develop new proteomic techniques to address these issues in a systematic and unbiased fashion.

We used cell surface biotinylation and solution-phase isoelectric focusing to extract and resolve plasma membrane proteins from murine T-cell hybridomas and primary splenocytes prior to identification using liquid chromatography tandem mass spectrometry (LC MS/MS). Using this method we identified 75 plasma membrane proteins, 13 of which appeared to be regulated following stimulation with phorbol 12-myristate 13-acetate and ionomycin. Six of these putative changes were verified independently by western blotting or by flow cytometry [4]. To develop protocols to examine macrophage signalling pathways we utilised antiphosphotyrosine antibodies to recover tyrosine phosphoproteins from RAW 264.7 cells activated with the tyrosine phosphatase inhibitor, pervanadate. Recovered proteins were eluted using phenyl phosphate, then resolved and identified by solutionphase isoelectric focusing and LC MS/MS. We identified 113 proteins inducibly associated with anti-phosphotyrosine immunoprecipitates from distinct subcellular compartments (cytosol, nucleus and plasma membrane), many of which were known tyrosine phosphoproteins (e.g. Fyn, Syk, SHIP, SLP76) but also included several hypothetical proteins, hitherto not documented at the protein level.

We then used this methodology to identify macrophage signalling proteins phosphorylated following cell contact with T-cell receptor transgenic splenocytes stimulated for 48 hours with specific (influenza virus hemaglutinin), or non-specific (ovalbumin), peptide. Proteins recovered in RAW cell phosphotyrosine immunoprecipitates were analysed by western blotting using antibodies to phosphotyrosine or the signalling molecules phospholipase $\mathrm{C}$ gamma, Hck, p38 and ERK. Cell contact with either resting or activated splenocytes induced acute and transient tyrosine phosphorylation of the src kinase Hck and phospholipase C gamma. In contrast, sustained activation of the MAP kinases p38 and ERK was observed only after contact with activated splenocytes.

Application of these methods should shed new light on the molecular bases of the contact-dependent activation of macrophages by activated $\mathrm{T}$ cells and thereby, suggest novel targets for therapeutic intervention in RA.

References

1. Brennan FM, Hayes $A L$, Ciesielski CJ, et al:: Evidence that rheumatoid arthritis synovial T cells are similar to cytokine-activated T cells: involvement of phosphatidylinositol 3-kinase and nuclear factor kappaB pathways in tumor necrosis factor alpha production in rheumatoid arthritis. Arthritis Rheum 2002, 46:31-41.

2. Burger $\mathrm{D}$, Dayer Jm: The role of human T-lymphocyte-monocyte contact in inflammation and tissue destruction. Arthritis Res 2002, 4 (Suppl 3):S169-S176

3. Mcinnes IB, Leung BP, Sturrock RD, et al: Interleukin-15 mediates cell-dependent regulation of tumor necrosis factor-alpha production in rheumatoid arthritis. Nat Med 1997, 3:189-195.

4. Peirce MJ, Wait R, Begum S, et al.: Expression profiling of lymphocyte plasma membrane proteins. Mol Cell Proteomics 2004, 3:56-65. 
P76

\section{Sustained downregulation of the TCR $\zeta$ chain defines a transition from antigen mode to inflammation mode during terminal T-cell differentiation}

Z Zhang, M Panesar, P Amjadi, A Foey, S Owen, F Dazzi, FM Brennan,
AP Cope

The Kennedy Institute of Rheumatology Division, Faculty of Medicine, Imperial College London, UK

Arthritis Res Ther 2005, 7(Suppl 1):P76 (DOI 10.1186/ar1597)

The molecular events that define early phases of activation and differentiation of effector $T$ cells have been well characterised. Those events regulating terminal differentiation and effector function of chronically activated $T$ cells at sites of inflammation, on the other hand, are less well understood. Using in vitro and in vivo models, we have explored the effects of the chronic inflammatory process on T-cell differentiation by investigating how tumour necrosis factor (TNF) regulates T-cell activation and effector responses. These studies have revealed that TNFstimulated $T$ cells resemble those derived from inflamed synovial joints since they express cell surface activation antigens, but are profoundly hyporesponsive to TCR engagement. Studies with mouse T cells suggest that this may be explained, at least in part, by the fact that TNF selectively targets the expression of the TCR $\zeta$ chain. Loss of TCR $\zeta$ expression perturbs the assembly, expression and stability of the TCR/CD3 complex leading to attenuation of membrane proximal tyrosine phosphorylation, intracellular calcium mobilisation and the transcription of cytokine genes upon TCR engagement, when compared with untreated T cells. We have begun to investigate whether TCR $\zeta$ expression could be used as a 'biomarker' for chronically activated, hyporesponsive T cells in human peripheral blood. Using a FACS-based assay, we have identified subsets of $\mathrm{CD}^{+} \mathrm{T}$ cells expressing low levels of TCR $\zeta$ (hereafter termed TCR $\zeta^{\text {dim }}$ cells) in the peripheral blood of healthy donors, as well as patients with inflammatory arthritis. In vitro studies of peripheral blood lymphocytes from healthy donors define not only antigen-dependent downmodulation of TCR $\zeta$ expression, which is transient, but also more sustained downregulation, which may arise through antigen-independent mechanisms. Experiments reveal that, when compared with TCR $\zeta^{\text {bright }}$ cells, the TCR $\zeta^{\text {dim }}$ population is enriched for cells expressing effector memory cell surface markers. While TCR $\zeta^{\text {dim }}$ cells are hyporesponsive to TCR engagement, they retain effector potential, since a significant proportion are capable of producing TNF- $\alpha$ and interferon gamma upon stimulation with phorbol ester and calcium

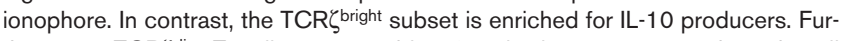
thermore, TCR $\zeta^{\text {dim }} \mathrm{T}$ cells are capable of activating monocytes through cell contact-dependent mechanisms. Together these data suggested that TCR $\zeta^{\text {dim }}$ T cells may represent a subset of circulating effector memory cells in vivo. Finally, we observed that TCR $\operatorname{dim} \mathrm{T}$ cells are enriched in inflamed joints of patients with inflammatory synovitis. Treatment of rheumatoid arthritis patients with anti-TNF (infliximab, $3 \mathrm{mg} / \mathrm{kg}$ ) leads to significant accumulation of TCR $\zeta^{\mathrm{dim}}$ T cells in peripheral blood of a subset of patients. Indeed, the extent of accumulation at 14 weeks after starting treatment predicts the DAS28 clinical response at 30 weeks. We propose that sustained downregulation of the TCR $\zeta$ chain defines a checkpoint where intracellular signals driving T-cell differentiation and effector responses switch from antigen mode to inflammation mode, where effector function may be largely antigen independent. As such this cell subset may represent a valid therapeutic cellular target.

Acknowledgments This work was funded by the Wellcome Trust and the Arthritis Research Campaign UK.

\section{P77}

\section{Rheumatoid synovial fibroblasts activate endothelial cells and promote neutrophil recruitment in a flow based multicellular model of the rheumatoid pannus}

\section{E Smith¹, F Lally', A Filer², M Stone³, J Shaw³, GB Nash¹, CD Buckley²,} GE Rainger ${ }^{1}$

${ }^{1}$ Department of Physiology, The Medical School, University of Birmingham, UK; ${ }^{2}$ Department of Rheumatology, The Medical School, University of Birmingham, UK; ${ }^{3}$ Respiratory and Inflammation Research, AstraZeneca, Alderley Park, UK Arthritis Res Ther 2005, 7(Suppl 1):P77 (DOI 10.1186/ar1598)

In rheumatoid arthritis, leukocytes are recruited from the blood by vascular endothelial cells (EC) and accumulate in stromal tissue forming ectopic leukocyte aggregates that contribute to soft tissue remodelling and bone destruction. The signals that regulate the process of chronic leukocyte recruitment into the diseased joint are poorly defined; however, it is probable that cells of the tissue stroma that have undergone a disease-specific transition in phenotype contribute to inflammation by the inappropriate production of proinflammatory cytokines and chemokines $[1,2]$. Here we have used a novel co-culture model [3] to monitor the role of stromal cells in regulating the quality and quantity of leucocytes that enter but not fibroblasts from normal tissue (skin), have the ability to drive inflammation by activating $\mathrm{EC}$ and promoting the recruitment of neutrophils.

EC were established in co-cultured with fibroblasts from the synovium (SF) or skin (DF) of RA patients on the opposite sides of porous transwell membranes. After 24 hours of EC conditioning, co-cultures were incorporated into a parallel plate leukocyte adhesion assay and the ability of $E C$ to recruit flowing neutrophils determined. Neutrophils adhered efficiently to EC co-cultured with $5 \times 10^{5} \mathrm{SF}$ $\left(88 \pm 12 / \mathrm{mm}^{2} / 10^{6}\right.$ cells perfused) but not to EC cocultured with DF $\left(12 \pm 9 / \mathrm{mm}^{2} / 10^{6}\right.$ cells perfused). Anti-P-selectin and anti-E-selectin antibodies markedly decreased neutrophil adhesion, and an anti-CD18 antibody (the $\beta 2$ integrin) abolished neutrophil recruitment. The antibody blockade of the neutrophil chemokine receptor CXCR2 but not CXCR1 also abolished neutrophil recruitment. Supernatants collected from cocultures of EC/SF, but not from SF cultured alone, activated EC to support neutrophil adhesion, indicating the presence of a soluble activating factor(s) generated exclusively in co-culture. EC/SF co-culture supernatants also contained a high concentration of IL-6 $(2925.2 \pm 521.2 \mathrm{pg} / \mathrm{ml})$, and both IL-6 levels and neutrophil adhesion were reduced in a dose-dependent manner by the inclusion of hydrocortisone in the co-culture medium. An anti-IL-6 antibody also abolished neutrophil adhesion.

We propose that in the rheumatoid synovium, a previously unsuspected process of cross-talk involving IL-6 signalling occurs between synovial fibroblasts and vascular EC resulting in the upregulation of adhesion molecules and chemokines that support neutrophil recruitment. The observation that neutrophil adhesion in this coculture system can be inhibited by anti-IL-6 neutralising antibodies or hydrocortisone suggests that this novel in vitro model of the synovium faithfully mimics important in vivo processes involved in leukocyte accumulation within the synovium. It also suggests that this new model can act as a useful screen for new anti-inflammatory therapies in rheumatoid arthritis.

References

1. Buckley CD, Filer A, Haworth O, Parsonage G, Salmon M: Defining a role for fibroblasts in the persistence of chronic inflammatory joint disease. Ann Rheum Dis 2004, 63(Suppl 2):ii92-ii95.

2. Parsonage G, Falciani F, Burman A, Filer A, Ross E, Bofill M, Martin S, Salmon M, Buckley CD: Global gene expression profiles in fibroblasts from synovial, skin and lymphoid tissue reveals distinct cytokine and chemokine expression patterns. Thromb Haemost 2003,90:688-697.

3. Rainger GE, Stone P, Morland CM, Nash GB: A novel system for investigating the ability of smooth muscle cells and fibroblasts to regulate adhesion of flowing leukocytes to endothelial cells. J Immunol Methods 2001, 255:73-82.

P78

Impaired early B-cell tolerance in patients with rheumatoid arthritis

J Samuels', Y-S Ng1, D Paget', E Meffre',

${ }^{1}$ Laboratory of Biochemistry and Molecular Immunology, Hospital for Special Surgery, New York, USA; 2 Weill Medical College of Cornell University, New York, USA

Arthritis Res Ther 2005, 7(Suppl 1):P78 (DOI 10.1186/ar1599)

Purpose Autoantibody production is a characteristic of most autoimmune diseases including rheumatoid arthritis (RA), but the stage of B-cell development at which B-cell tolerance is broken remains unknown. We previously established in healthy donors that most autoreactive developing $B$ cells were removed either in the bone marrow or in the periphery, revealing two B-cell tolerance checkpoints. We aimed to determine whether the autoreactive B cells in RA evade one or both of these checkpoints. In addition, analysis of the antibody repertoires in RA might lead to potential insights about the mechanisms that may explain loss of B-cell tolerance.

Methods We obtained peripheral blood from four patients with clinically and serologically documented active RA, who had not yet received steroids, disease-modifying anti-rheumatic drugs or biologic therapy, or who had been off such regimens for at least 4 months. After isolating their peripheral B cells, we sorted, cloned and expressed in vitro antibodies from single new emigrant $\left(\mathrm{CD} 19^{+} \mathrm{CD} 10^{+} \mathrm{lgM}+\mathrm{CD} 27^{-}\right)$ and mature naive $\left(\mathrm{CD}_{19}{ }^{+} \mathrm{CD} 10^{-} \mathrm{IgM}^{+} \mathrm{CD} 27^{-}\right) \mathrm{B}$ cells. In total, we examined by ELISA the specificity of 232 recombinant antibodies.

Results The antibody sequences in two of the patients showed a significant increase in the proportion of 11 amino-acid long CDR3s in IgK chains (previously described in RA synovium and blood), while the other two patients demonstrated an increase in $\mathrm{J}_{\kappa} 1$ usage along with a shift towards more downstream $\mathrm{V}_{\kappa}$ segments. This skewed lgK repertoire suggests that receptor editing, one of the mechanisms that ensures B-cell tolerance, is defective in those two patients. Despite the different antibody sequence patterns of our RA patients, all four patients showed significantly increased proportions of polyreactive antibodies in the new emigrant compartment $(23.1 \%$ versus $8.2 \%$ in controls, $P=0.0094$ ) autoreactive antibodies in the mature naïve compartment $(45.3 \%$ versus $20.4 \%$ in controls, $P=0.0001)$, and anti-CCP antibodies in both compartments (13.8\% versus $0 \%$ in controls, $P=0.019$ ). This demonstrates that autoreactive $B$ cells in RA patients fail to be removed at both checkpoints of early B-cell tolerance. 
Conclusions RA patients exhibit defective central and peripheral B-cell tolerance, which may favor the development of autoimmunity. We have also identified two distinct groups of RA patients based on their antibody sequences that suggest divergent mechanisms within the RA population - yet lead to a common phenotype.

\section{P79}

\section{Characterization of the IL-12 family of cytokines in human dendritic cells infected with live Chlamydia trachomatis MK Matyszak, JC Goodall, JSH Gaston}

University of Cambridge Clinical School, Department of Medicine,

Addenbrooke's Hospital, Cambridge, UK

Arthritis Res Ther 2005, 7(Suppl 1):P79 (DOI 10.1186/ar1600)

Introduction Chlamydia trachomatis (CT) is a clinically important pathogen. In $2-5 \%$ of cases, infection results in the development of reactive arthritis. We have previously shown that human dendritic cells (DC) can be readily infected with live CT following in vitro exposure [1]. Infected DC efficiently process chlamydia and present chlamydial antigens to both $\mathrm{CD}^{+}{ }^{+}[1]$ and $\mathrm{CD} 8^{+} \mathrm{T}$ cells $[1,2]$. Infected DC also produce a number of cytokines including IL-12 and tumour necrosis factor alpha but not IL-10 [1]. The production of IL-12 following infection gives DC the ability to stimulate Th1 responses to chlamydia. Here we have investigated production of another two cytokines from the IL-12 family - IL-23 and IL-27 by DC infected with live CT. The results were compared with those obtained following DC activation with other inflammatory stimuli.

$\mathrm{IL}-23$ is a heterodimer composed of $\mathrm{p} 19$ and p40 subunits, where the p40 subunit is shared between IL-23 and IL-12. IL-27 is a heterodimer composed of p28 and Epstein-Barr virus-induced protein 3 (EBI3) subunits. The main function of both of these cytokines is to stimulate interferon gamma (IFN- $\gamma$ ) production in T cells. However, whereas IL-27 activity primarily affects naïve T cells, IL-23 stimulates both primed and memory cells.

Methods DC were generated in vitro from $\mathrm{CD} 14^{+}$monocytes purified from peripheral blood mononuclear cells. After 7 days of culture in the presence of GM-CSF and IL-4, DC were fully differentiated. Immature DC, DC activated with lipopolysaccharide (LPS), IFN- $\gamma$, heat-killed CT and infected with live CT were used for these experiments. Total RNA was purified using the High Pure RNA isolation kit (Roche). IL-23 oligos used were: p19 forward, $5^{\prime}$-tcg-gca-cga-gaa-caactg-ag-3'; p19 reverse, 5'-tgg-gga-aca-tca-ttt-gta-gtc-t-3'; p40 forward, $5^{\prime}$-att-gag-gtc-atg-gtg-gat-gc-3'; p40 reverse, 5'-att-gct-ggc-att-ttt-gcg-gc-3'. IL27 oligos used were: p28 forward, $5^{\prime}$-gcg-gaa-tct-cac-ctg-cca-g-3'; p28 reverse, 5'-cgg-gag-gtt-gaa-tcc-tgc-a-3'; EBI3 forward, 5'-cgt-gcc-ttt-cat-aac-aga-gca-3'; EBI3 reverse, 5'-gac-gta-gta-cct-ggc-tcg-g-3'. GAPDH was used as control. PCR settings: $95^{\circ} \mathrm{C}$ for 3 min followed by 29 cycles of $95^{\circ} \mathrm{C}$ for $30 \mathrm{sec}, 60^{\circ} \mathrm{C}$ for $30 \mathrm{sec}, 72^{\circ} \mathrm{C}$ for $90 \mathrm{sec}$. PCR products were resolved on $1.5 \%$ agarose gel. Results We showed that infection of DC with live CT resulted in the upregulation of message for all four subunits studied (p19, p40, p28, EBI3). The message was detected at 18 and 24 hours post infection. LPS-activated DC upregulated $p 28$, $\mathrm{p} 40$ and EBI3 but not p19. There was no p19 message at either 18 or 24 hours post stimulation. The main subunit upregulated following IFN- $\gamma$ activation was p28 and there was no detectable p19 mRNA at any of the times studied. Interestingly, there was also no expression of $\mathrm{p} 19$ when immature DC were exposed to heatkilled CT. Exposure of DC to heat-killed CT did, however, upregulate p28, p40 and EBI3. A small amount of $\mathrm{EBI} 3$ message was detected in immature $\mathrm{DC}$, but on none of the other subunits studied.

Conclusion Whereas activation of DC with LPS, IFN- $\gamma$ and heat-killed CT stimulates production of IL-27, these stimuli are insufficient to induce p19 upregulation, and in consequence IL-23 production. However, infection of DC with live CT resulted in the production of mRNA for both IL-23 and IL-27. This study highlights important differences in immune responses following exposure to live and heat-killed CT.

\section{References}

1. Matyszak MK, Young J, Gaston JS: Uptake and processing of Chlamydia trachomatis by human dendritic cells. Eur J Immuno/ 2002, 32:742-751.

2. Matyszak MK, Gaston JS: Chlamydia trachomatis-specific human CD8+ T cells show two patterns of antigen recognition. Infect Immun 2004, 72:4357-4367.

\section{P80}

\section{NKT cell status and IL-10-dependent therapeutic effect of} NKT cell stimulation on collagen-arthritis in DBA/1 mice N Bessis, A Miellot, A Herbelin, R Zhu, M-C Boissier

Université Paris 13, France

Arthritis Res Ther 2005, 7(Suppl 1):P80 (DOI 10.1186/ar1601)

Background and objectives Defective NKT cell function has been linked with autoimmunity. Both the number of NKT cells and their functional capacity of releasing interferon gamma (IFN- $\gamma$ ) and IL-4 after TCR ligation are for instance impaired in NOD mice developing diabetes, in mice developing a model of multiple sclerosis and also in humans with autoimmune diseases. To investigate the NKT cell role in rheumatoid arthritis, we studied their quantitative and qualitative profile in collagen-induced arthritis (CIA) DBA/1 susceptible mice, and we tested whether NKT stimulation with their synthetic ligand alpha-galactosylceramide $(\alpha-$ GalCer) was therapeutic in CIA.

Methods The number of NKT cells in the liver was determined by flow cytometry with an anti-TCR $\alpha \beta$ or empty or $\alpha$-GalCer-loaded CD1 tetramer. CIA was induced by immunization of DBA/1 mice with collagen II (CII) in adjuvant, and with a boost 21 days later. $\alpha$-GalCer $(4 \mu \mathrm{g})$ was administered at the same time of the first immunization. In one experiment, one group of mice was also treated with an anti-IL-10 receptor antibody. Cll-specific CD4 ${ }^{+}$cells from lymph node (LN) response was monitored by immunizing mice with $75 \mu \mathrm{g} \mathrm{Cll}$ in adjuvant in hind paws. Some mice were administered $4 \mu \mathrm{g} \alpha$-GalCer in the $\mathrm{Cll} /$ adjuvant mixture at the same time. Nine days after immunization, $\mathrm{CD}^{+}{ }^{+}$cells were purified from LN and cultured with CII and antigen-presenting cells. Proliferation was measured after 3 days by measuring BrdU incorporation, and IL-4, IL-10 and IFN- $\gamma$ levels were assessed by ELISA in the supernatants.

Results The number of NKT cells among leucocytes in the liver of DBA/1 mice was comparable to what is generally observed in $\mathrm{C} 57 \mathrm{BI} / 6$ and suggest a normal quantitative profile of NKT cells in DBA/1 mice. In contrast, in vivo NKT cell function was altered in DBA/ 1 mice since stimulation with $\alpha$-GalCer ( $4 \mu \mathrm{g}$ intraperitoneally) led to decreased IL-4 and IFN- $\gamma$ levels in the serum 2 hours after the injection, as compared with C57BI/6 mice $(693 \pm 154 \mathrm{pg} / \mathrm{ml}$ versus $1557 \pm 137 \mathrm{~g} / \mathrm{ml} \mathrm{IL-4}$ $[P<0.01]$ and $2077 \pm 378$ versus $4005 \pm 581 \mathrm{IFN}-\gamma[P<0.02])$. Treatment of $\mathrm{ClA}$ with $\alpha$-GalCer at day 0 induced a clear-cut diminution of clinical (ANOVA test, $P=0.0001)$ and histological scores $(1.19 \pm 0.14$ versus $1.85 \pm 0.24, P<0.05)$ of arthritis, as compared with the control group. Importantly, treatment of mice with an anti-IL-10 receptor abrogated the protective effect of $\alpha$-GalCer. The $\alpha$-GalCerinduced protection was associated with the ability of $\mathrm{LN} \mathrm{CD} 4^{+}$cells from Cll-immunized and $\alpha$-GalCer-treated DBA/ 1 mice to secrete larger amounts of IL-10 upon in vitro restimulation with $\mathrm{Cll}$, while IL-4 and IFN- $\gamma$ levels were not affected. Cllinduced proliferation was slightly reduced in LN CD4 cells from Cll-immunized and $\alpha$-GalCer-treated DBA/1 mice as compared with controls.

Conclusion These findings raise the possibility that NKT stimulation might induce a shift toward an anti-inflammatory Th2 status and could be used therapeutically to treat chronic autoimmune arthritis.

\section{P81}

\section{Distinct patterns of RANKL/osteoprotegerin system} modulation through anti-tumour necrosis factor and corticosteroid therapy in rheumatoid arthritis synovium Al Catrina1, D Makrygiannakis', E af Klint' ${ }^{1}$, SB Catrina², S Ernestam ${ }^{3}$, L Klareskog', AK Ulfgren'

${ }^{1}$ Department of Rheumatology, Karolinska University Hospital, Solna, Karolinska Institutet, Stockholm, Sweden; ${ }^{2}$ Department of Molecular Medicine, Karolinska University Hospital, Solna, Karolinska Institutet, Stockholm, Sweden; ${ }^{3}$ Department of Rheumatology, Karolinska University Hospital, Huddinge, Karolinska Institutet, Stockholm, Sweden

Arthritis Res Ther 2005, 7(Suppl 1):P81 (DOI 10.1186/ar1602)

Background Anti-tumour necrosis factor (TNF) therapy with both etanercept and infliximab decreases radiographic progression of patients with rheumatoid arthritis (RA), while the effect of local corticosteroid injections, a routine adjuvant therapy in arthritis, on bone metabolism is still debated. Thus, we investigated the effect of both anti-TNF and local corticosteroid therapy on synovial expression of osteoprotegerin (OPG) and receptor activator of NF- $\mathrm{kB}$ ligand (RANKL).

Methods OPG and RANKL were evaluated by immunohistochemistry in serial synovial biopsies obtained from 18 RA patients before and after 8 weeks of treatment with etanercept (nine patients) or infliximab (nine patients). Eighteen additional patients with arthritis that received a local corticosteroid injection were evaluated before and after 2 weeks of the injection. Biopsies were evaluated by double-blind semi-quantitative analysis and image analysis. The in vitro effect of TNF antagonists and corticosteroids (dexametasone) on RANKL/OPG expression in osteoblasts was evaluated by western blot. Statistical analysis was performed using the Wilcoxon's signed-rank test followed by Bonferroni correction. Results OPG was present in all biopsies with a characteristic pattern restricted mainly to the endothelial cells and few mononuclear cells. RANKL was present mainly in the T-cell area and to a lesser extent on some endothelial cells, but absent in other mononuclear cells. Treatment with both infliximab and etanercept increased synovial OPG expression. Neither infliximab nor etanercept influenced RANKL expression following 8 weeks of treatment. The RANKL/OPG ratio decreased following therapy in both groups, the effect being more pronounced in the responders as compared with non-responders to therapy. Local corticosteroid treatment resulted in a similar change of the RANKL/OPG ratio through a different mechanism, with a significant decrease of the synovial RANKL and no changes in the OPG expression. In vitro both TNF antagonists and corticosteroids mimicked the in vivo effect inducing a decrease in the RANKL/OPG ratio in TNF-primed osteoblasts. 
Conclusion Therapy with both TNF antagonists and local corticosteroids modulates the RANKL/OPG system, inhibiting bone destruction through distinct mechanisms. Thus, association of these two therapies may be beneficial in preventing bone erosions in RA.

P82

IL-1-driven cartilage and bone destruction are dependent on Toll-like receptor (TLR) 4, but not TLR2

S Abdollahi-Roodsaz, LAB Joosten, MF Roelofs, MMA Helsen, B Oppers-Walgreen, CHM Arndtz, TRDJ Radstake, WB van den Berg

Rheumatology Research Laboratory and Advanced Therapeutics, University Medical Center Nijmegen, The Netherlands

Arthritis Res Ther 2005, 7(Suppl 1):P82 (DOI 10.1186/ar1603)

Background: Rheumatoid arthritis (RA) is an autoimmune disease characterized by systemic, chronic joint inflammation. In spite of great efforts, the etiology of RA has not been completely elucidated to date. Toll-like receptors (TLRs) belong to the family of pattern recognition receptors and are involved in both innate and adaptive immune responses against microorganisms via recognition of pathogenassociated molecular patterns. In addition, TLRs have been reported to recognize several endogenous ligands, which are generated under stress conditions and cartilage damage. Triggering of TLRs results in the release of various proinflammatory cytokines such as IL-1 $\beta$ and tumor necrosis factor alpha. IL-1 $\beta$ is one of the crucial cytokines in the pathogenesis of RA and is involved in Th1-mediated processes and cartilage and bone destruction. Therefore, TLRs may potentially be involved in either initiation or chronicity of arthritis.

Objective To study the contribution of TLR2 and TLR4 in IL-1 $\beta$-driven joint inflammation, cartilage destruction and bone erosion.

Methods C57BL/6 wild-type (WT), BL/6 TLR2 knockout (KO), C57BL/10 WT and BL/10 TLR4 KO mice were intra-articularly injected with $3 \times 10^{6} \mathrm{PFU}$ mouse IL-1 $\beta$ adenoviral vector (AdmlL-1 $\beta$ ) or control vector (Ad5del70-3). Inflammatory cell infiltration, cartilage proteoglycan $(\mathrm{PG})$ depletion and cartilage and bone destruction were microscopically examined 7 days after virus injection.

Results Prolonged expression of mIL-1 $\beta$ in mice knee joints induced a severe arthritis in WT animals, exhibiting pathophysiologic changes resembling those in human RA. High amounts of inflammatory cells, predominantly polymorphonuclear cells, were present in the knee joint cavities of both WT and KO mice. Severe PG depletion, cartilage destruction and bone erosion were observed in WT mice, which did not differ from those in TLR2-deficient mice. Interestingly, in TLR4-deficient mice, cartilage PG depletion and destruction were significantly reduced. TLR4 KO mice showed also a marked reduction in bone erosion compared with WT mice, although this reduction was not significant.

Conclusion These data strongly suggest that TLR4 is involved in IL-1 $\beta$-driven pathologic processes. Both cartilage and bone destruction were reduced in TLR4 KO mice, although joint inflammation was comparable with WT mice. This indicates the involvement of potential TLR4 ligands, generated by IL-1 $\beta$-induced cartilage degradation, in chronic RA. Our data point out that TLR4 may be a novel therapeutic target in the treatment of RA.

Acknowledgements Supported by the Dutch Arthritis Association. AdmIL-1 $\beta$ virus was a kind gift from Dr Carl D Richards from the Department of Pathology and Molecular Medicine, McMaster University, Ontario, Canada.

P83

\section{Differential expression of multidrug resistance-related proteins on monocyte-derived macrophages from rheumatoid arthritis patients}

R Oerlemans ${ }^{1}$, BAC Dijkmans', JW van der Heijden'1, WF Lems', A Reurs², GL Scheffer ${ }^{2}$, RJ Scheper ${ }^{2}$, G Jansen ${ }^{1}$

${ }^{1}$ Department of Rheumatology, VU University Medical Center, Amsterdam, The Netherlands; ${ }^{2}$ Department of Pathology, VU University Medical Center, Amsterdam, The Netherlands

Arthritis Res Ther 2005, 7(Suppl 1):P83 (DOI 10.1186/ar1604)

Background Primary or acquired drug resistance is well recognized as a common cause of treatment failure of patients with cancer. From a rheumatoid arthritis (RA) perspective, the issue of resistance to anti-rheumatic drugs (e.g. disease-modifying anti-rheumatic drugs [DMARDs]) has not received much attention. A possible mechanism of drug resistance is overexpression of multidrug resistance (MDR)-related proteins. The objective of this study was to identify whether various MDR proteins are expressed on macrophages as potential target cells for DMARDs.

Methods Monocyte-derived macrophages (MDM) were obtained from 15 RA patients (median age: 56 years, 13 females/two males) and eight healthy controls (median age: 51 years, four females/four males). Monocytes were isolated from peripheral blood mononuclear cells and differentiated into macrophages in the presence of monocyte-colony stimulating factor. Cytospins of MDMs were prepared for immunohistological analysis of the MDR-related proteins: P-glycopro- cancer resistance protein (BCRP) and lung resistance protein (LRP). Staining intensities were scored as follows: (0), negative; (1), weakly positive; (2), positive; (3), strongly positive. The total score is depicted as the summation of percentiles of cells with intensities $0-3$.

Results MDMs of healthy controls and of RA patients stained strongly positive for macrophage markers CD68 and 3A5. Except for MRP4, MDR protein expression was observed both in MDMs of healthy controls and RA patients. Results are shown as the median score with ranges (in parentheses) for MDR expression and a statistical evaluation (Mann-Whitney $U$ test) for differential expression (Table 1).

Table 1

Multidrug resistance (MDR) protein expression in monocyte-derived macrophages from rheumatoid arthritis patients and healthy controls as median scores (ranges)

\begin{tabular}{lccc} 
MDR protein & $\begin{array}{c}\text { Rheumatoid arthritis } \\
\text { patients }(n=15)\end{array}$ & Controls $(n=8)$ & $P$ value \\
\hline BCRP & $1.04(0.51-2.27)$ & $0.63(0.44-1.45)$ & 0.043 \\
LRP & $0.87(0-2.49)$ & $0.64(0.1-1.35)$ & 0.876 \\
MRP1 & $0.36(0-1.03)$ & $0.25(0.09-1.01)$ & 0.309 \\
MRP5 & $0.31(0-1.04)$ & $0.12(0.05-0.78)$ & 0.238 \\
P-glycoprotein & $0.24(0-1.05)$ & $0.1(0.05-1.06)$ & 0.813 \\
MRP4 & $<0.05$ & $<0.05$ & \\
\hline
\end{tabular}

Conclusions MDR proteins, known to be involved in conferring drug resistance, are expressed on macrophages of RA patients. Beyond this, there seems to be a trend for a higher expression of MDR proteins on MDM from RA patients compared with healthy controls. Further studies are warranted to assess whether MDR is a clinically relevant issue in daily treatment of RA patients.

Acknowledgement This study is supported by the Dutch Arthritis Association (Grant NRF-03-I-40).

\section{P84}

Inhibition of tumour necrosis factor alpha production by activated $\mathbf{T}$ cells of rheumatoid arthritis patients by novel anti-folate drugs: an ex vivo pilot study

JW van der Heijden', AH Gerards'2, R Oerlemans', WF Lems', RJ Scheper ${ }^{3}$, LA Aarden 4 , BAC Dijkmans'1, G Jansen'

${ }^{1}$ Department of Rheumatology, Vrije Universiteit Medical Center, Amsterdam, The Netherlands; ${ }^{2}$ Vlietland Hospital, Schiedam, The Netherlands; ${ }^{3}$ Department of Pathology, Vrije Universiteit Medical Center, Amsterdam, The Netherlands; ${ }^{4}$ Department of Immunopathology, Sanquin-Research, Amsterdam, The Netherlands Arthritis Res Ther 2005, 7(Suppl 1):P84 (DOI 10.1186/ar1605)

Introduction The folate antagonist methotrexate (MTX) is the 'anchor-drug' in the treatment of patients with rheumatoid arthritis (RA) [1]. The main target of MTX in intracellular folate metabolism is dihydrofolate reductase (DHFR) but several other targets have been described (e.g. thymidylate synthase [TS] and 5-aminoimidazole-4-carboxamide ribonucleotide [AICAR]). At present the exact mechanism of action of MTX in RA still remains elusive [1]. Despite the potent anti-rheumatic capacity of MTX many patients (at least $50 \%$ ) become resistant to MTX during long-lasting therapy. However, little is known about the mechanisms of resistance against MTX in RA patients [2].

From the field of oncology, where MTX is used against haematological malignancies, new anti-folate drugs were developed to circumvent MTX resistance [3]. These new folate antagonists have the following characteristics: are better transported through the reduced folate carrier, are retained intracellular more efficiently by polyglutamylation via folylpolyglutamate synthetase, and/or have other targets in the folate pathway besides DHFR (e.g. TS).

Objective To investigate whether two new-generation anti-folate drugs, PT523 (DHFR-inhibitor) and ZD1694 (TS-inhibitor), have equal or better anti-inflammatory capacity compared with MTX based on their capacity to inhibit tumour necrosis factor alpha (TNF- $\alpha$ ) production by activated T cells.

Methods Whole blood from 11 RA patients and six healthy volunteers was incubated ex vivo with MTX, PT523, ZD1694 and, as a control, the DMARD sulphasalazine (SSZ) after T-cell stimulation with $\alpha$-CD3/CD28. Inhibition of TNF- $\alpha$ production was measured after 72 hours by ELISA ${ }^{[4]}$.

The IC-50 values (defined as the drug concentration exerting $50 \%$ inhibition of TNF- $\alpha$ production) are used as a value for the anti-inflammatory capacity of the drug (Table 1). 
Table 1

Characteristics of drugs and mean IC-50 values ( \pm standard deviation)

\begin{tabular}{|c|c|c|c|c|c|}
\hline \multirow[b]{2}{*}{ Drug } & \multirow[b]{2}{*}{ Target } & \multirow[b]{2}{*}{$\begin{array}{l}\text { Reduced } \\
\text { folate } \\
\text { carrier } \\
\text { affinity } \\
\text { (cell uptake) }\end{array}$} & \multirow[b]{2}{*}{$\begin{array}{c}\text { Folylpoly- } \\
\text { glutamate } \\
\text { synthetase } \\
\text { affinity } \\
\text { (cell retention) }\end{array}$} & \multicolumn{2}{|c|}{$\begin{array}{l}\text { Mean IC-50 for } \\
\text { TNF- } \alpha \text { inhibition }\end{array}$} \\
\hline & & & & $\begin{array}{c}\text { Rheumatoid } \\
\text { arthritis } \\
\text { patients } \\
(n=11)\end{array}$ & $\begin{array}{l}\text { Controls } \\
(n=6)\end{array}$ \\
\hline MTX & DHFR & ++ & + & $78(77) \mathrm{nM}$ & 57 (27) nM \\
\hline PT523 & DHFR & +++ & - & $5.8(3.2) \mathrm{nM}$ & 6,4 (8.3) nM \\
\hline ZD1694 & TS & +++ & +++ & $16(26) \mathrm{nM}$ & $4.8(3.0) \mathrm{nM}$ \\
\hline SSZ & $N F-\kappa B$ & - & - & $278(216) \mu \mathrm{M}$ & $191(29) \mu \mathrm{M}$ \\
\hline
\end{tabular}

Results Both PT523 and D1694 turned out to inhibit TNF- $\alpha$ production by activated T cells much more efficiently than MTX (5-15 times). For comparison, the DMARD SSZ is effective at much higher concentration ( $\mu \mathrm{M}$ range). The inhibition of TNF- $\alpha$ production by the anti-folate drugs does not seem to be a result of (apoptotic) cell death of T cells whereas SSZ induces apoptosis of T cells (data not shown).

Conclusion In an ex vivo setting, two novel anti-folate drugs designed to circumvent MTX resistance proved to be very effective in inhibiting TNF- $\alpha$ production by activated T cells from RA patients and healthy volunteers. Future experiments are designed to evaluate ex vivo anti-folate sensitivity profiles for 'MTX-responders' and 'MTX-non-responders' to investigate whether these novel generation of antifolate drugs can be useful in cases of clinical failure on MTX.

References

1. Kremer JM: Toward a better understanding of methotrexate. Arthritis Rheum 2004, 50:1370-1382.

2. Jansen G, Scheper RJ, Dijkmans BAC: Multidrug resistance proteins in rheumatoid arthritis, role in disease modifying antirheumatic drug efficacy and inflammatory processes: an overview. Scand J Rheumatol 2003, 32:325-339.

3. Rots MG, Pieters R, Peters GJ, van Zantwijk CH, Mauritz R, Noordhuis P, Willey JC, Hahlen K, Creutzig U, Janka-Schaub G, et al:: Circumvention of methotrexate resistance in childhood leukemia subtypes by rationally designed antifolates. Blood 1999, 94:3121-3128.

4. Gerards AH, de Lathouder S, de Groot ER, Dijkmans BAC, Aarden LA: Inhibition of cytokine production by methotrexaat. Studies in healthy volunteers and patients with rheumatoid arthritis. Rheumatology 2003, 42:1189-1196.

Acknowledgement This study is supported by the Dutch Arthritis Association (Grant NRF-03-I-40)

\section{P85}

Marked expression of IL-1 receptors in cell nuclei and muscle fiber membrane in muscle tissue of patients with idiopathic inflammatory myopathies

C Grundtman', S Salomonsson', C Dorph1, J Burton², U Andersson'3,

\section{IE Lundberg'}

${ }^{1}$ Department of Rheumatology, Karolinska University Hospital Solna, Karolinska Institutet, Stockholm, Sweden; ${ }^{2}$ Department of Physiology, Karolinska Institutet, Stockholm, Sweden; ${ }^{3}$ Department of Woman and Child Health, Karolinska University Hospital Solna, Karolinska Institutet, Stockholm, Sweden

Arthritis Res Ther 2005, 7(Suppl 1):P85 (DOI 10.1186/ar1606)

Background In chronic inflammatory diseases, IL-1 is known as an important proinflammatory cytokine inducing the expression of a variety of genes mediating the synthesis of several proteins, which can induce both acute and chronic inflammatory changes. Idiopathic inflammatory myopathies, such as polymyositis and dermatomyositis, are chronic autoimmune muscle disorders characterised by proximal muscle weakness and histopathological signs of patchy distribution of inflammatory cells in the skeletal muscle. Muscle tissue of patients with such myopathies has been shown to have an increased expression of IL-1 $\alpha$ and IL-1 $\beta$; however, the expression and pathophysiological role of IL-1 receptors in muscle tissue from such patients and healthy subjects has not yet been elucidated.

Aim To investigate the expression of IL-1 receptors in muscle tissue from both patients and healthy subjects and to describe whether IL-1 receptors are differentially expressed in symptomatic and non-symptomatic muscle tissue of patients with polymyositis and dermatomyositis.

Method Muscle biopsies from eight patients with polymyositis, three patients with dermatomyositis, and six healthy subjects were included in this study. Muscle biopsies were taken from two different sites, one from a symptomatic muscle and another biopsy from a non-symptomatic muscle. IL-1 $\alpha$, IL-1RI, and IL-1RII expression were investigated by immunohistochemistry. IL-1RI and IL-1RII were also investigated by confocal microscopy.

Results In patients with polymyositis and dermatomyositis, IL-1 $\alpha$ was found to be expressed in endothelial cells and inflammatory cells, and significantly increased in symptomatic when compared with non-symptomatic muscle tissue. IL-1 $\alpha$ was not expressed in muscle tissue of healthy subjects. In patients, IL-1RI and IL-1RII were strongly expressed in inflammatory cells, endothelial cells, in the membrane of the muscle fiber, as well as in the nucleus of the muscle fiber of patients, whereas in muscle tissue of healthy subjects only a scattered pattern of IL-1RI and IL-1RII expression in a few endothelial cells and in a few of nuclei of the muscle fiber could be observed. The membrane and nuclear expression were confirmed by double stainings.

Discussion This is the first study to show the expression of IL-1RI and IL-1RII in the cell nuclei and in the membrane of muscle fibers. Moreover, we have found some marked differences between symptomatic and non-symptomatic muscle tissue of patients and healthy subjects indicating a potentially important role of IL-1 receptors in the pathogenesis of idiopathic inflammatory myopathies.

\section{P86}

Interleukin-1 receptor antagonist lentiviral gene transfer to the murine knee joint ameliorates collagen-induced arthritis FAJ van de Loo, LM Delavallee, OJ Arntz, MB Bennink, LAB Joosten, WB van den Berg

Rheumatology Research \& Advanced Therapeutics, Department of Rheumatology, University Medical Center Nijmegen, The Netherlands Arthritis Res Ther 2005, 7(Suppl 1):P86 (DOI 10.1186/ar1607)

Introduction The inflamed joints in rheumatoid arthritis patients are ideally suited for gene therapy applications to acquire local production of potent anti-inflammatory biologics. The need for precise and absolute targeting as for treating cancer is not necessary in rheumatoid arthritis. However, the challenge is to obtain longterm transgene expression. For this purpose we used in this study HIV-based lentiviruses (LV) for the stable transduction of the murine synovium.

Methods The coding sequence of murine interleukin-1 receptor antagonist (IL-1Ra) was inserted into the pRRLcpptpgkmcspreSsin transfer vector (selfinactivating vector was a gift from D Trono, Geneva, Switzerland) behind the phosphoglycerate kinase-promoter. Vesicular stomatitis virus $G$ envelope pseudotyped lentiviruses were produced by cotransfection of 293T-cells [1]. Recombinant lentiviruses were purified and titres were determined using the p24 ELISA (Murex HIV Antigen MAB, Abbott). Lentiviruses encoding for firefly luciferase (Luc) were used for control. The lentiviruses were injected intra-articularly into the murine knee joint. Synovial explants were taken to measure the luciferase activity using a luminometer. Mice immunized against bovine collagentype II received an intra-articularly injection of lentiviruses 1 day after the antigen booster but without any visual signs of collagen-induced arthritis.

Results Naîve C57BI/6 mice were injected with $300 \mathrm{ng}$ (p24 value) of LV-Luc into the knee joint. The Luc activity in the synovial biopsies increased steadily until day 7 and remained at this level until the end of the experiment (day 21 after injection). Next we injected the same amount of LV-mlL-1Ra and LV-Luc into the right knee joint of collagen-type II immunized mice just before onset of arthritis. At day 31 after immunization all mice in the LV-IL-1Ra and LV-Luc-treated groups developed clinical signs of severe arthritis in the paws. However, macroscopic examination of the injected knees showed a significant reduction in joint inflammation in all mice of the LV-mIL-1Ra group (arbitrary score [0-3] $0.55 \pm 0.37$ ) as compared with the LV-Luc-treated mice (score $1.55 \pm 0.53$ ). Microscopic evaluation confirmed the marked reduction in joint inflammation. Furthermore, the joint that received LV-mIL-1Ra showed a marked reduction in bone destruction whereas the extent of cartilage destruction was comparable with the LV-Luctreated knees during collagen-induced arthritis.

Conclusion We show for the first time that lentiviral-mediated local overexpression of IL-1Ra had a pronounced effect on the development of collagen-induced arthritis in that particular knee joint. No protective effect was observed on the ipsilateral or contralateral paws as we have demonstrated previously for IL-1Ra gene transfer using adenoviral vectors $[2,3]$. The lentiviral transduction of the synovium seems feasible and efficacious for treatment of arthritis.

References

1. Dull T, Zufferey R, Kelly M, Mandel RJ, Nguyen M, Trono D, Naldini L: A third-generation lentivirus vector with a conditional packaging system. $J$ Virol 1998, 72:8463-8471.

2. Bakker AC, Van de Loo FA, Joosten LA, Bennink MB, Arntz OJ, Dmitriev IP, Kashentsera EA, Curiel DT, van den Berg WB: A tropism-modified adenoviral vector increased the effectiveness of gene therapy for arthritis. Gene Ther 2001, 8:1785-1793.

3. Bakker AC, van de Loo FA, Joosten LA, Arntz OJ, Varley AW, Munford RS, van den Berg WB: C3-Tat/HIV-regulated intraarticular human interleukin-1 receptor antagonist gene therapy results in efficient inhibition of collagen-induced arthritis superior to cytomegalovirus-regulated expression of the same transgene. Arthritis Rheum 2002, 46:1661-1670. 
P87

Effects of anti-rheumatic treatments on the prostaglandin $E_{2}$ biosynthetic pathway in synovial tissue and synovial fluid cells from patients with rheumatoid arthritis

M Korotkova, M Westman, KR Gheorghe, E af Klint, C Trollmo,

A-K Ulfgren, L Klareskog, P-J Jakobsson

Department of Medicine, Rheumatology Unit, Karolinska Institutet/Karolinska University Hospital, Stockholm, Sweden

Arthritis Res Ther 2005, 7(Suppl 1):P87 (DOI 10.1186/ar1608)

Background Microsomal prostaglandin E synthase (mPGES)- 1 is upregulated in experimental arthritis and is markedly expressed in synovial tissue from patients with rheumatoid arthritis (RA), suggesting its important role of in the pathogenesis of inflammatory arthritis. However, the effects of current anti-rheumatic therapies on mPGES-1 expression have not been examined.

Objective To study the effects of anti-tumour necrosis factor (TNF) alpha blockers and glucocorticoids on prostaglandin (PG) $E_{2}$ biosynthesis and mPGES-1 expression in synovial fluid mononuclear cells (SFMC) and synovial tissue from RA patients. Methods Synovial tissues were obtained from 18 RA patients before and after treatment with TNF-blockers (infliximab and etanercept) and from 16 patients before and after intra-articular injection of steroids. SFMC were obtained from eight RA patients. In vitro effects of TNF-blockers and dexamethasone (Dex) on mPGES-1 expression in SFMC were examined by flow cytometry. PGE $_{2}$ levels in culture supernatants were analyzed by enzyme immunoassay. Immunohistological analysis and double immunofluorescence were performed using antibody against mPGES-1, cyclooxygenase (COX) and CD163.

Results Treatment of SFMC with TNF-blockers or Dex decreased lipopolysaccharide-induced mPGES-1 and COX-2 expression in CD14+ monocytes and $\mathrm{PGE}_{2}$ synthesis in culture supernatants. Double immunofluorescence revealed that mPGES-1 and COX-2 were co-localized in SFMC and in synovial tissue cells. Local treatment with steroids significantly reduced the mPGES-1, COX-2 and COX-1 expression in synovial tissue. However, neither mPGES-1 nor COX-2 expression in RA synovial tissues were significantly affected by anti-TNF therapy. Conclusion In vitro, both TNF-blockers and Dex suppressed mPGES-1 in SFMC. In RA synovial tissue, local steroids but not TNF-blockade downregulate mPGES1. These data provide support to the use of combination of TNF-blockade and inhibitors of $\mathrm{PGE}_{2}$ production for optimal anti-inflammatory achievement.

\section{P88}

Mitogen-activated kinases in psoriatic arthritis: differences in activation and distinct regulation by etanercept therapy RJU Lories, I Derese, FP Luyten, $K$ de Vlam

Laboratory for Skeletal Development and Joint Disorders, Department of Rheumatology, University Hospitals Leuven, Katholieke Universiteit Leuven, Belgium Arthritis Res Ther 2005, 7(Suppl 1):P88 (DOI 10.1186/ar1609)

Introduction Mitogen-activated protein kinases (MAPKs) are important effector enzymes of different molecular signaling cascades with critical roles in inflammation, in particular in the induction and downstream effects of tumor necrosis factor (TNF) alpha and IL-1. Inhibition of the MAPK signaling cascades has been identified as a potential therapeutic target in chronic arthritis. Little is known about the activation and extent of MAPK signaling in the synovium of patients with psoriatic arthritis. We studied phosphorylation of the three common MAPKs (p38, extracellular regulating kinase [ERK] and C-jun-N-terminal kinase [JNK]) in psoriatic arthritis and the effect of anti-TNF therapy on their activation.

Materials and methods Synovial biopsies were obtained by needle arthroscopy in nine patients with active psoriatic arthritis in a clinically involved knee before the initiation of etanercept (Enbrel ${ }^{\mathbb{C}}$; Wyeth Pharmaceuticals, Louvain-la-Neuve, Belgium). All patients were included in a 6-month open-label clinical trial of $25 \mathrm{mg}$ etanercept two times a week [1]. Follow-up biopsies were taken in the same knee after 6 months. Six biopsies were used for histological examination. Frozen sections were stained with $\mathrm{H} \& \mathrm{E}$ or used for immunofluorescense. Sections were fixed with acetone and stained with goat-anti-human phosphorylated ERK, mouse-anti-human phosphorylated p38 or mouse-anti-human phosphorylated JNK (all from Santa Cruz Technologies, Santa Cruz, USA). Cy2-conjugated donkey anti-goat or Сy3-conjugated goat anti-mouse antibodies (Jackson Immunoresearch, West Grove, USA) were used as secondary antibodies. Negative controls were performed with non-specific IgG. Severity of synovitis was assessed with a composite score based on a blinded semi-quantitative score $(0-3)$ of four parameters (lining layer thickness, sublining vascularity, inflammatory cell infiltration and presence of lymphoid aggregates). Activation of MAPKs was quantified with Image J digital analysis software. The area of fluorescent staining due to antibody-linked fluorochrome emission was normalized to cell number based on area of emission by DAPI nuclear staining. For statistical analysis non-parametric Wilcoxon test for paired samples was used.

Results Activated p38 was present in both lining and sublining layers. In the sublining layer, positive cells were found in inflammatory infiltrates, in perivascular zones and in mononuclear cell infiltrates and perivascularly. Activation of JNK was recognized in cells of the lining layer, in some sublining cell infiltrates and the endothelium. The composite histological severity score was significantly lower after etanercept treatment (median score 4 versus $1 ; P<0.02$ ). Etanercept therapy resulted in a significant decrease in ERK (median normalized fluorescent area 0.096 versus $0.04 ; P<0.03$ ) and JNK (median normalized fluorescent area 0.91 versus $0.34 ; P<0.03$ ) but not in p38 activation (median normalized fluorescent area 0.04 versus $0.07 ; P>0.07$ ).

Conclusion Different MAPKs are activated in psoriatic arthritis synovium. The activation pattern suggests distinct roles for specific MAPKs in synovitis. The activation pattern of $\mathrm{p} 38$ and pERK strongly mimics the pattern described in rheumatoid arthritis [2]. Activation of JNK in the lining layer of rheumatois arthritis synovium has not been reported. Etanercept therapy had a significant effect on JNK and ERK but not on p38 activation. These data suggest that other, probably TNF-independent, signals activate p38 in psoriasis arthritis synovium.

References

1. de Vlam K, Lories RJ: Treatment of refractory polyarticular psoriatic arthritis with etanercept in monotherapy [abstract]. Ann Rheum Dis 2004, 63(Suppl 1):S405.

2. Schett G, et al:: Activation, differential localization, and regulation of the stress activated protein kinases ERK, JNK and p38 MAPK in synovial tissue and cells in rheumatoid arthritis. Arthritis Rheum 2000, 43:2501 2512.

Acknowledgements $\mathrm{KdV}$ is the recipient of an unrestricted medical school grant from Wyeth Pharmaceuticals. RL is a post-doctoral fellow from the Fund for Scientific Research Flanders.

\section{P89}

Identification of a natural soluble form of the IL-18 receptor accessory protein as an immunomodulator in experimental arthritis

\section{RL Smeets, OJ Arntz, MB Bennink, WB van den Berg, FAJ van de Loo}

Experimental Rheumatology and Advanced Therapeutics, Department of Rheumatology, University Medical Center Nijmegen, The Netherlands

Arthritis Res Ther 2005, 7(Suppl 1):P89 (DOI 10.1186/ar1610)

Background In the inflammatory process preceding erosive arthritis, IL-18 plays an important role. IL-18 is known to regulate immune responses by stimulating Th1 maturation, and signaling is initiated through formation of a trimericc receptor complex, consisting out of IL-18 bound to the IL-18R $\alpha$ and its accessory receptor IL-18R $\beta$.

Objective The aim of this study was to determine the physiological role of a recently described soluble form of the IL-18 receptor accessory protein (sIL-18R $\beta$ ) in mice. Methods Mouse sIL-18R $\beta$ (genebank accession number AK053176) was cloned from murine lung CDNA and used for the generation of an adenoviral vector (Ad.5IL-18R $\beta$ ). Expression analysis of the sIL-18R $\beta$ and its full-length membrane bound IL-18R $\beta$ in different murine tissue was achieved through endpoint PCR. To investigate the in vivo mode of action, sIL-18R $\beta$ was systemically overexpressed in collagen type II-immunized male DBA/1 mice. Systemical overexpression was achieved through intravenous injection of $3 \times 10^{8}$ pfu Ad5sIL-18R $\beta$ or the control vector (Ad5Luc) before clinical manifestation of collagen-induced arthritis (CIA). At 1 and 4 days post adenoviral injection, splenocytes were harvested and the cytokine profile in plasma and splenocyte culture supernatants was determined Results Short IL-18R $\beta$ mRNA was highly expressed in tissue of lymphoid origin, and no expression could be observed in immune privileged organs like the testis, the eye and the brain, suggesting a prominent role in immune regulation. Expression of sIL-18R $\beta$ was disease regulated in mice suffering from CIA, whereas the full-length IL-18R $\beta$ was not regulated. Splenocytes of slL-18R $\beta$-treated immunized mice produced significantly less interferon gamma and IL-10 compared with control treated animals. Adenoviral overexpression of the sIL-18R $\beta$ before clinical manifestation of CIA significantly aggravated arthritis, which was accompanied by a reduction of circulating IL-10, interferon gamma and a significant increased anti-bovine collagen II $\lg G_{1}$.

Conclusion Our results describe the existence of a novel short soluble form of the membrane IL-18R $\beta$, which is mainly expressed in lymphoid tissues. This sIL$18 R \beta$ expression appears regulated during CIA. Furthermore, we show that this novel soluble IL-18R $\beta$ functions as a putative modulator of IL-18 signaling; aggravating $\mathrm{CIA}$, by modulating T-cell immunity.

\section{P90}

Gene expression in chronically activated Th cells

U Niesner', I Albrecht', R Baumgrass'1, M Janitz ${ }^{2}$, H Lehrach ${ }^{2}$, T Haeupl ${ }^{3}$, J Grun', GR Burmester ${ }^{3}$, A Grutzkau', A Radbruch'

${ }^{1}$ German Arthritis Research Centre, Berlin, Germany; ${ }^{2}$ Department of Vertebrate Genomics, Max Planck Institute for Molecular Genetics, Berlin, Germany;

${ }^{3}$ Department of Rheumatology and Clinical Immunology, Charité University Medical Centre, Berlin, Germany; ${ }^{4}$ Oligene Inc., Berlin, Germany

Arthritis Res Ther 2005, 7(Suppl 1):P90 (DOI 10.1186/ar1611)

Th lymphocytes are considered to be key players in chronic inflammation. Upon repeated re-stimulation and differential instruction, they can develop a stable 


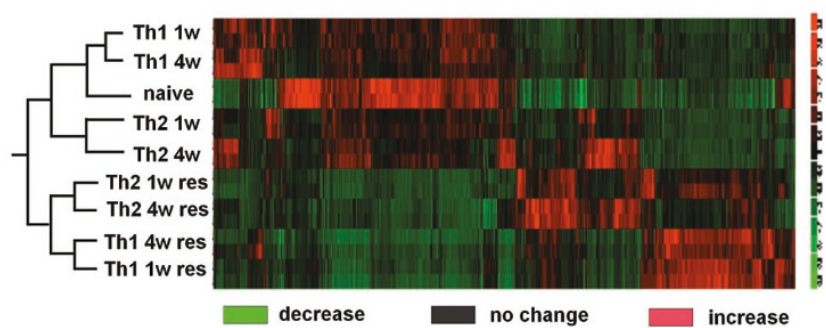

Hierarchical clustering of differentially expressed genes among doublets of the nine investigated cell populations ( $w$, weeks in culture; res, 3 hours re-stimulated) using Affymetrix Mouse Genome 430A 2.0 Arrays and DNA-Chip Analyzer (dChip).

memory for the expression of distinct genes coding for cytokines, chemokines, adhesion molecules, and so on. This functional memory contributes considerably to the chronicity of inflammation and its eventual refraction to therapy.

Here, we describe the global gene expression profiles of in vitro repeatedly reactivated murine Th1 and Th2 cells, and compare them with those of naïve and once activated Th1 and Th2 cells, both before and after re-stimulation (Fig. 1).

Among differentially expressed genes is a novel transcription factor, which downmodulates NF-kB signalling and is exclusively expressed in repeatedly re-activated Th1 cells. High expression of this gene can be detected in Th cells isolated from the site of inflammation of patients suffering from distinct forms of rheumatic diseases or ulcerative colitis, but not in Th cells isolated from healthy tissue or from blood. Expression of this gene in Th cells at the site of inflammation highlights the role of chronic T-cell activation in chronic inflammation and may represent an endogenous mechanism to limit inflammation. Apart from its diagnostic value, this molecular pathway also points to a new therapeutic target.

\section{P91}

Intracellular IL-1 receptor antagonist (iclL-1Ra1) does not antagonize growth inhibitory effects of pre-IL-1 $\alpha$ in SaOS-2 cells

\section{G Palmer, S Trolliet, D Talabot-Ayer, F Mézin, D Magne, C Gabay}

Division of Rheumatology, University Hospital of Geneva and Department of Pathology and Immunology, University of Geneva School of Medicine, Geneva, Switzerland

Arthritis Res Ther 2005, 7(Suppl 1):P91 (DOI 10.1186/ar1612)

Background IL- $1 \alpha$ is synthesized as a precursor (prelL-1 $\alpha$ ), which is processed into mature IL- $1 \alpha$ and a $\mathrm{N}$-terminal propeptide by calpain-like proteases. Besides its classical effects elicited upon IL-1 receptor binding, prelL- $1 \alpha$ exerts intracellular functions, including the modulation of cell growth and apoptosis. Nuclear translocation of prelL- $1 \alpha$, mediated by the $\mathrm{N}$-terminal propeptide, is required for these effects. IL-1 receptor antagonist (IL-1Ra) inhibits the classical effects of IL-1 by preventing the interaction of IL-1 with its receptor. Four different isoforms of IL-1Ra have been described, of which one is secreted and three others are intracellular (iclL-1Ra1, iclL-1Ra 2, iclL-1Ra 3). Due to their intracellular localization, iclL-1Ras cannot interact with cell surface receptors and have been suggested to carry out specific functions inside cells. The description of nuclear functions for prelL-1 $\alpha$ suggested that iclL1Ra variants might antagonize intracellular effects of prelL-1 $\alpha$. Objective and methods The aim of this study was to investigate effects of prelL$1 \alpha$ and iclL-1Ra1 on cell growth using stably transfected SaOS-2 osteosarcoma cells. IL- $1 \alpha$ and IL-1Ra expression was quantified by ELISA and their localization was examined by immunofluorescence. Cell counts, lactate dehydrogenase activity and $\left[{ }^{3} \mathrm{H}\right]$-thymidine incorporation were used to monitor cell growth.

Results IL- $1 \alpha$ expression ranged from 5.3 to $9.4 \mathrm{ng} / 10^{6}$ cells in culture supernatants and from 39.8 to $87.3 \mathrm{ng} / 10^{6}$ cells in lysates of prelL- $1 \alpha$ transfected SaOS- 2 cells. Immunostaining showed nuclear localization of prelL- $1 \alpha$, which was not modified by co-transfection of iclL-1Ra1. Expression levels of IL-1Ra ranged from 53 to $219 \mathrm{ng} / 10^{6}$ cells in supernatants and from 1678 to 4414 $\mathrm{ng} / 10^{6}$ cells in lysates of iclL-1Ra1 transfected cells. IL-1Ra staining was essentially cytoplasmic. Transfection of SaOS- 2 cells with prelL- $1 \alpha$ significantly decreased cell growth, as indicated by reduced cell counts and lactate dehydrogenase activity. PrelL- $1 \alpha$ also decreased thymidine incorporation, indicating an inhibition of cell proliferation. In contrast, addition of exogenous mature IL-1 $\alpha$ $(500 \mathrm{pg} / \mathrm{ml})$ had no inhibitory effect on SaOS-2 proliferation, suggesting that the effect of prelL- $1 \alpha$ on cell growth is intracellular. Transfection of SaOS- 2 cells with iclL-1Ra1 alone did not affect cell growth. Moreover, co-expression of iclL$1 \mathrm{Ra} 1 \mathrm{did}$ not reverse the growth inhibitory effect of prelL-1 $\alpha$. However the induction of IL- 6 secretion, a classical, receptor-mediated effect of prelL- $1 \alpha$, was inhibited in iclL-1Ra1 co-transfected cells.
Conclusions Expression of prelL- $1 \alpha$ decreased the growth of SaOS-2 cells. Cotransfection of iclL-1Ra1 did not antagonize this effect. These observations suggest that intracellular effects of prelL- $1 \alpha$ are not necessarily susceptible to inhibition by iclL-1Ra.

\section{P92}

Expression of microsomal prostaglandin E synthase-1 in muscle tissue of patients with idiopathic inflammatory myopathies

\section{Korotkova, S Barbasso, P-J Jakobsson, I Lundberg}

Rheumatology Unit, Department of Medicine, Karolinska Institutet/Karolinska University Hospital, Stockholm, Sweden

Arthritis Res Ther 2005, 7(Suppl 1):P92 (DOI 10.1186/ar1613)

Background Microsomal prostaglandin E synthase (mPGES)-1 catalyzes the formation of prostaglandin (PG) $\mathrm{E}_{2}$ from cyclooxygenase (COX)-derived $\mathrm{PGH}_{2}$. mPGES-1 is induced by proinflammatory cytokines, such as $\mathrm{IL}-1 \beta$ and tumor necrosis factor alpha, and is linked to conditions with high $\mathrm{PGE}_{2}$ biosynthesis and inflammation. $\mathrm{A}$ recent study has demonstrated enhanced COX-1 and COX-2 expressions in affected muscle tissues of patients with polymyositis (PM) and dermatomyositis (DM), suggesting their important role in the pathophysiology of these diseases. However, expression of mPGES-1 in muscle tissue has not been investigated.

Objective To study the expression of mPGES-1 in muscle tissue of patients with idiopathic inflammatory myopathies.

Methods Muscle biopsies were obtained from 13 patients with idiopathic inflammatory myopathies (six PM, four DM, one juvenile DM, two inclusion body myositis) and from six healthy subjects. Immunohistochemical analysis was performed using antibodies against mPGES-1.

Results mPGES-1 staining was detected in all patients with PM, DM and inclusion body myositis. Specifically strong intracellular staining was observed in inflammatory cells in infiltrates, in endothelial cells of capillaries, in scattered mononuclear cells and in fibroblast-like cells. In some patients mPGES-1 staining was localized in smooth muscle cells and endothelial cells of large vessels. In healthy subjects, weak mPGES-1 staining was detected only in scattered fibroblast-like cells, in a few mononuclear cells surrounding large vessels and in some capillaries.

Conclusion These results demonstrate the upregulation of mPGES-1 in muscle tissue of refractory patients with idiopathic inflammatory myopathies compared with healthy controls, suggesting a role of mPGES-1 in the pathophysiology of these diseases.

\section{P93}

\section{Quantifying the contribution of the second HLA-DRB1 susceptibility allele to the risk of rheumatoid arthritis: a meta-analysis}

\section{IA van Rossum 1, AH Zwinderman'2, PP Tak', N de Vries'}

${ }^{1}$ Department of Clinical Immunology \& Rheumatology, AMC/University of Amsterdam, The Netherlands; ${ }^{2}$ Division of Medical Statistics, AMC/University of Amsterdam, The Netherlands

Arthritis Res Ther 2005, 7(Suppl 1):P93 (DOI 10.1186/ar1614)

Background $H L A-D R B 1$ alleles encoding the shared epitope (SE) susceptibility sequence predispose to rheumatoid arthritis (RA). Having two copies results in even higher risk. This study analyzes whether the contribution of the second SE allele to RA risk is identical to that of the first SE allele.

Methods In a meta-analysis of 44 datasets of case-control studies meeting predefined criteria, the odds ratios $\mathrm{OR}_{1 / 0}$ and $\mathrm{OR}_{2 / 1}$ are calculated, as an estimate of the relative risk for RA for individuals having one compared with zero, or two compared with one $\mathrm{SE}$ allele; from these, the ratio $\mathrm{OR}_{2 / 1}: \mathrm{OR}_{1 / 0}$ is calculated.

Results Overall the weighted mean ratio $\mathrm{OR}_{2 / 1}: \mathrm{OR}_{1 / 0}(95 \%$ confidence interval) was 0.90 (0.80-1.01). In the 39 studies in which all patients met the ACR criteria for $\mathrm{RA}$, the overall ratio $\mathrm{OR}_{2 / 1}: \mathrm{OR}_{1 / 0}$ is $0.89(0.78-1.01$; see Fig. 1$)$. In a multiple regression analysis on a group level the ratio $\mathrm{OR}_{2 / 1}: \mathrm{OR}_{1 / 0}$ was not influenced by racial origin, the frequency of compound heterozygosity (0401/0404 or 0401/0408), the percentage of female or rheumatoid factor-positive patients, disease duration or age at disease onset.

Conclusions The contribution of the second SE allele to RA risk does not differ significantly from that of the first SE allele. This result will help to pool immunogenetic data across populations, resulting in a detailed description of the contribution of immunogenetic factors to RA risk.

In addition this finding implies that pathogenetic models for RA that incorporate the role of HLA should explain the fact that the first and the second SE alleles increase RA risk equally. Such effects might, for example, be described for models incorporating presentation of pathogenic antigenic peptides. 
Figure 1

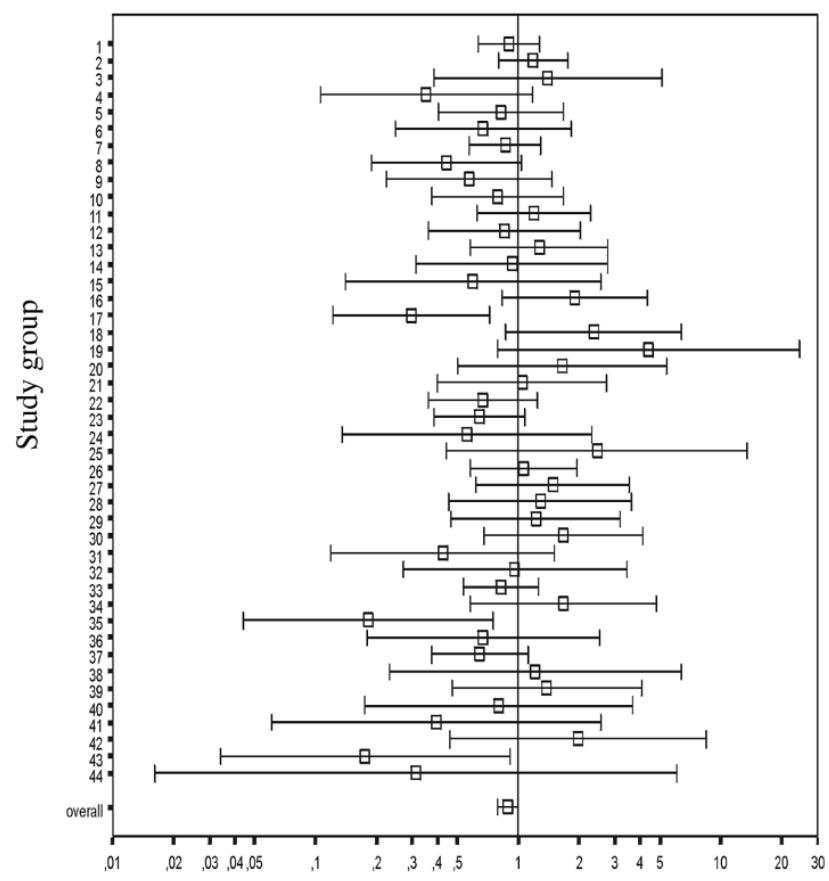

Ratio $\mathrm{OR}_{2 / 1}: \mathrm{OR}_{1 / 0}$ (see Methods) with $95 \%$ confidence interval of the 44 study groups and the overall weighted mean.

\section{P94}

Efficient IL-18 and IL-18BPc adenoviral gene transfer to cultured murine submandibular gland epithelial cells and successful murine retrograde submandibular duct cannulation to modulate IL-18 function in the salivary gland of animal models of Sjogren's syndrome

M Bombardieri'1,2, F Barone ${ }^{1,2}$, G Proctor ${ }^{3}$, FA van de Loo ${ }^{4}$, WB van den Berg ${ }^{4}$, G Valesini ${ }^{2}$, IB McInnes ${ }^{5}$, C Pitzalis ${ }^{1}$

${ }^{1}$ Rheumatology Department, GKT School of Medicine, KCL, London, UK; ${ }^{2}$ Rheumatology Unit, University of Rome 'La Sapienza', Italy; ${ }^{3}$ Salivary Research Group, GKT School of Dentistry, KCL, London, UK; ${ }^{4}$ Rheumatology Research and Advanced Therapeutics, Department of Rheumatology, University Medical Center Nijmegen, The Netherlands; ${ }^{5}$ Centre for Rheumatic Diseases, Glasgow Royal Infirmary, Glasgow University, UK

Arthritis Res Ther 2005, 7(Suppl 1):P94 (DOI 10.1186/ar1615)

Introduction We recently demonstrated that the proinflammatory and immunoregulatory cytokine IL-18 is strongly upregulated in salivary glands (SG) of Sjogren's syndrome (SS) patients. IL-18 gain or loss of function experiments in animal models of human diseases have provided evidence of the pathogenic role of this cytokine in chronic inflammation. However, in SS the pathogenic relevance of IL-18 in the development of murine autoimmune sialoadenitis has not as yet been evaluated. Here we describe in vitro experiments in which murine SG epithelial cells were tested for transfection efficiency with IL-18 and IL$18 \mathrm{BPcAdV}$ gene transfer. In addition, in preparation for in vivo $\mathrm{SG}$ modulation of IL-18 function by AdV-mediated gene transfer, we optimised retrograde SG cannulation in mice.

Methods Primary SG ductal epithelial cells (SG-DEC), established from submandibular glands of C57BL/6 mice using the explant outgrowth technique, as well as a murine SG-DEC line were used for AdV transfection. A mIL-18AdV from plasmid IL-18 TG3652 [1] was developed in our laboratory while we used the same mIL-18BPcAdV construct as previously reported [2]. AdVs encoding for LacZ or luciferase were used as controls. All AdVs were incubated at $50 \mathrm{MOI}$. Efficiency of gene transfer was evaluated through detection of IL-18 and IL$18 \mathrm{BPc}$ protein expression assessed by both immunostaining and western blot. Beta-galactosidase staining was performed to evaluate efficiency of transfection of LacZAdV. Feasibility of local delivery of compounds through retrograde cannulation of murine submandibular glands was also tested.

Results High efficiency of transfection of cultured murine SG-DEC was obtained
Figure 1

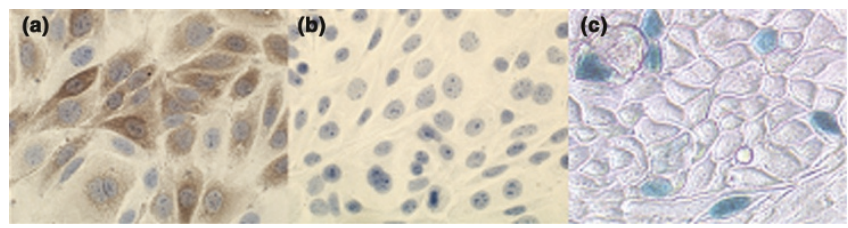

(a) Expression of murine IL-18 in salivary gland ductal epithelial cells (SG-DEC) transfected with IL-18AdV but not with LacZAdV (b). (c) Beta-galactosidase staining confirmed effective transfection of SG-DEC by LacZAdV.

\section{Figure 2}

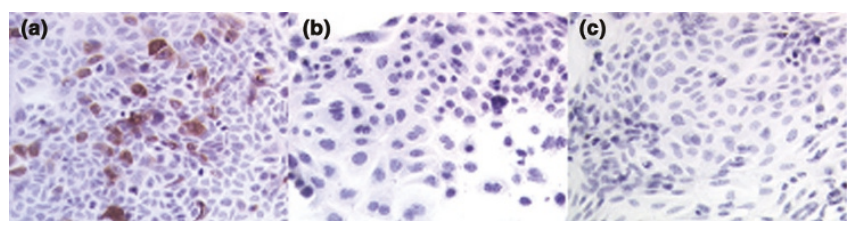

(a) Expression of murine IL-18BPc in salivary gland ductal epithelial cells transfected with IL-18BPcAdV but not with LucAdV (b) or medium alone (c).

the presence of protein production detectable as single bands from targetgeneAdV but not control-geneAdV transfected SG-DEC. A time-course study demonstrated in vitro gene expression up to 3 weeks after transfection. Feasibility of local SG delivery through retrograde submandibular duct cannulation was demonstrated by injection of trackable compounds.

Conclusion Here we report for the first time evidence of high and sustained efficiency of IL-18 and IL-18BPc AdV gene transfer in murine SG-DEC. In addition, we successfully adapted a cannulation technique previously used in bigger animals for in vivo local delivery of modulatory molecules to murine salivary glands.

Local delivery of IL-18/IL-18BPc adenoviral vectors in vivo in salivary glands of NOD mice and other murine models of SS through retrograde submandibular excretory duct cannulation will provide evidence of a possible pathogenic role of IL-18 in participating in autoimmune sialoadenitis and will establish a rationale for using IL-18 blocking agents as therapeutic tools in SS.

References

1. Walter DM, Wong CP, DeKruyff RH, Berry GJ, Levy S, Umetsu DT: II-18 gene transfer by adenovirus prevents the development of and reverses established allergen-induced airway hyperreactivity. J Immunol 2001, 166:6392-6398.

2. Smeets RL, van de Loo FA, Arntz OJ, Bennink MB, Joosten LA, Van Den Berg WB: Adenoviral delivery of IL-18 binding protein $C$ ameliorates collagen-induced arthritis in mice. Gene Ther 2003, 10:1004-1011.

\section{P95}

Type 1 regulatory $T$ cells in treatment of murine lupus

R Undeutsch'1, J Humrich', BH Hahn², F Hiepe', G Burmester',

A Radbruch ${ }^{3}$, G Riemekasten ${ }^{1}$

${ }^{1}$ Charité University Hospital, Berlin, Germany; ${ }^{2}$ University of California, Los Angeles, California, USA; ${ }^{3}$ German Arthritis Research Centre, Berlin, Germany Arthritis Res Ther 2005, 7(Suppl 1):P95 (DOI 10.1186/ar1616)

Systemic lupus erythematosus is a severe systemic autoimmune disease characterized by loss of tolerance towards a restricted panel of autoantigens. As a result, pathogenic autoantibodies against dsDNA or the Sm proteins occur. We identified the SmD1(83-119) peptide, the C-terminus of the spliceosomal protein SmD1, as a major B-cell and T-cell autoantigen in human and murine lupus [1-3]. In previous work we could show that intravenous high-dose application of SmD1(83-119) prolongs survival in NZB/W F1 mice and delays occurrence of anti-dsDNA autoantibodies [4]. Higher percentages of $\mathrm{CD} 4^{+} \mathrm{T}$ cells that produce $\mathrm{IL}-10$ and interferon gamma were detected on restimulation with phorbol 12myristate 13-acetate/ionomycin later on in the spleen, indicating involvement of type 1 regulatory $\mathrm{T}$ cell ( $\operatorname{Tr} 1$ cell)-mediated tolerance [4]. Transfer of splenic $\mathrm{CD} 0^{+} \mathrm{T}$ cells from mice treated with high doses of SmD1(83-119) into untreated mice delayed the occurrence of anti-dsDNA autoantibodies in these recipients as well [4].

We now performed a SmD1(83-119) specific analysis of the CD4+ $\mathrm{T}$ cells after high-dose application of SmD1(83-119) and detected SmD1(83-119)-reactive $\mathrm{CD} 4^{+} \mathrm{IL}-10^{+} \mathrm{Tr} 1$ cells in the spleen and in draining lymph nodes after additional 
immunization with SmD1(83-119). In vitro experiments showed that Tr1-cellmediated suppression of anti-DNA autoantibody production is dependent on the activity of IL-10 as the addition of neutralizing anti-IL-10 antibodies abrogated this effect. Furthermore, adoptive transfer of SmD1(83-119) reactive Tr1-cell-containing lymph node cells delayed the occurrence of anti-DNA autoantibodies in the recipient mice as well.

We conclude that high-dose application of SmD1(83-119) induces SmD1 (83-119) specific tolerance in NZB/W F1 mice, which is mediated by SmD1(83-119)reactive Tr1 cells. These results may open new ways for future autoantigen specific cell-based therapies in systemic lupus erythematosus.

References

1. Riemekasten G, et al.: A novel epitope on the C-terminus of SmD1 is recognized by the majority of sera from patients with systemic lupus erythematosus. J Clin Invest 1998, 102:754-763.

2. Riemekasten G, et al.: T cell reactivity against the SmD1(83-119) C terminal peptide in patients with systemic lupus erythematosus. Ann Rheum Dis 2002, 61:779-785.

3. Riemekasten G, et al.: Identification and characterization of SmD183119-reactive $T$ cells that provide $T$ cell help for pathogenic anti-doublestranded DNA antibodies. Arthritis Rheum 2003, 48:475-485.

4. Riemekasten G, Langnickel D, et al:: Intravenous injection of a D1 protein of the smith proteins postpones murine lupus and induces type 1 regulatory T cells. J Immunol 2004, 173:5835-5842.

Acknowledgements $\mathrm{RU}$ and $\mathrm{JH}$ contributed equally to this work.

\section{P96}

Hearing improvement in a variant Muckle-Wells syndrome case in response to IL-1 receptor antagonism

\section{Rynne, C Maclean, MF McDermott, P Emery}

Academic Unit of Musculoskeletal Disease, Department of Rheumatology, Leeds General Infirmary, Leeds, UK

Arthritis Res Ther 2005, 7(Suppl 1):P96 (DOI 10.1186/ar1617)

Background The proinflammatory cytokine IL-1 $\beta$ has been implicated in the pathogenesis of a number of the hereditary periodic fever syndromes. One such syndrome, Muckle-Wells syndrome (MWS), is characterised by the triad of urticaria, progressive sensorineural deafness and systemic amyloid A amyloidosis. Other features include rigors, leucocytosis, raised acute phase reactants and serum amyloid A levels. A number of case reports have recently emerged involving treatment with the recombinant human IL-1 receptor antagonist, Anakinra (Kineret; Amgen, Cambridge, UK) [1-3].

Case report A 59-year-old caucasian female presented with increasingly severe and intractable disease over a 15-year period. In addition to the above features, she also exhibited papilloedema and chronic aseptic meningitis. No other family members were affected. Upon commencing treatment with Anakinra, there was complete resolution of her inflammatory symptoms within 24-48 hours, and rapid normalisation of her C-reactive protein and serum amyloid A levels (from $415.0 \mathrm{mg} / \mathrm{l}$ to $12.6 \mathrm{mg} / \mathrm{l}$ after 4 weeks of therapy). Her intracranial pressure and CSF white cell counts also returned to normal. Audiometry confirmed a $15-30$ decibel improvement in the $250-4000 \mathrm{~Hz}$ frequency range in each ear. No mutations of the responsible gene - NALP3/CIAS1 on chromosome 1q44 were demonstrated on her DNA sequencing.

Discussion Our patient is the oldest reported sporadic case of MWS. Heterozygous missense mutations have thus far been reported in only $60 \%$ of MWS patients analysed [4]. The confirmed improvements in hearing, intracranial pressure, and CSF white cell counts seen here with Anakinra lend further support to the treatment of the autoinflammatory conditions by targeting IL-1. The pathogenesis of the sensorineural deafness in MWS is unclear although it is postulated that expression of mutated NALP3/CIAS1 in cartilage may have a causative role [3].

\section{References}

1. Hoffman HM, Patel DD: Genomic-based therapy: targeting interlerukin1 for autoinflammatory diseases. Arthritis Rheum 2004, 50:345-346.

2. Hawkins PN, Lachmann HJ, McDermott MF: Interleukin-1 receptor antagonist in Muckle-Wells syndrome. N Engl J Med 2003, 348:2583-2584.

3. Hawkins PN, Lachmann HJ, Aganna E, McDermott MF: Spectrum of clinical features in Muckle-Wells syndrome and response to Anakinra. Arthritis Rheum 2004, 50:607-612.

4. Neven B, Callebaut I, Prieur AM, Feldmann J, Bodemer C, Lepore L, Derfalvi B, Benjaponpitak S, Vesely R, Sauvain MJ, et al.: Molecular basis of the spectral expression of CIAS1 mutations associated with phagocytic cell-mediated autoinflammatory disorders CINCA/NOMID, MWS, and FCU. Blood 2004, 103:2809-2815.

Acknowledgements The authors gratefully acknowledge the invaluable assistance of Alison Bybee (Royal Free and University College Medical School, London) and Ebun Aganna (Queen Mary's School of Medicine and Dentistry, London) for their sequencing of our patient's DNA.
P97

The cytokine memory of SmD183-119-autoantigen-specific Th cells of systemic lupus erythematosus patients S Langer ${ }^{1}$, D Langnickel', P Enghard ${ }^{1}$, GR Burmester ${ }^{1}$, F Hiepe $^{1,2}$, G Riemekasten'

${ }^{1}$ Department of Rheumatology and Clinical Immunology, Charité University Hospital, Humboldt University of Berlin, Germany; ${ }^{2}$ German Rheumatism Research Center, Berlin, Germany

Arthritis Res Ther 2005, 7(Suppl 1):P97 (DOI 10.1186/ar1618)

Objective The aim of the study was to analyze the cytokine memory of Th cells derived from systemic lupus erythematosus (SLE) patients and healthy donors enriched for autoantigen-specific T cells by in vitro stimulation with SmD183-119, a common autoantigen in SLE.

Method Autoreactive $\mathrm{CD}^{+} \mathrm{T}$ cells derived from 37 SLE patients and 14 healthy donors were enriched by repetitive ex vivo stimulation of peripheral blood mononuclear cells with SmD183-119. For control, peripheral blood mononuclear cells were stimulated only with IL-2. After two rounds of antigen stimulations, cultures were stimulated with phorbol 12-myristate 13-acetate/ionomycin for intracellular cytokine staining. Frequencies of cytokine-expressing $T$ cells were analyzed and, in SLE patients, compared with disease activities and autoantibody levels.

Results Comparing cultures from SLE patients with those from healthy donors, SLE patients displayed higher frequencies of tumor necrosis factor alpha-positive T cells and the frequencies correlated with disease activity. SmD183-119-induced tumor necrosis factor alpha expression correlated with serum anti-dsDNA antibody levels.

The frequencies of IL-10-expressing $T$ cells were lower in cultures from SLE patients. Cultures from patients with high frequencies of IL-10+ $\mathrm{T}$ cells revealed low disease activities. SmD183-119-induced increases in IL-10-expressing T cells were associated with low anti-dsDNA and anti-SmD183-119 antibody levels in culture supernatants.

Conclusion The enrichment of SmD183-119-reactive $\mathrm{T}$ cells by in vitro cultures showed that cytokine changes specifically occur in lupus patients. The results give insight into the cytokine memory of autoreactive Th cells and their role in lupus pathogenesis.

\section{P98}

\section{PTPN22 as a rheumatoid arthritis susceptibility but not severity gene}

J Wesoly1, VEH Carlton'2, A Chokkalingam², AHM van der Helm-van Mil1, REM Toes' ${ }^{1}$ AB Begovic ${ }^{2}$, TWJ Huizinga ${ }^{1}$

${ }^{1}$ Department of Rheumatology, Leiden University Medical Center, The

Netherlands; ${ }^{2}$ Celera Diagnostics, Alameda, California, USA

Arthritis Res Ther 2005, 7(Suppl 1):P98 (DOI 10.1186/ar1619)

Background Due to the complex multigenic nature of rheumatoid arthritis (RA) identification of RA susceptibility and severity genes appears to be more challenging than originally anticipated. Among many recently reported RA susceptibility genes, a missense single nucleotide polymorphism in the protein tyrosine phosphatase PTPN22 appears to be involved in susceptibility to multiple autoimmune diseases including diabetes, systemic lupus erythematosus and Graves disease. Whether this polymorphism is also associated with severity of the phenotype in diseased individuals is not known.PTPN22 encodes a hematapoetic phosphatase also known as Lyp that functions as a negative regulator of T-cell activation via interaction with the c-Src tyrosine kinase Csk and phosphorylation of regulatory tyrosines or other Src family kinases, such as Lck and Zap70. The R620W polymorphism results in substitution of conserved arginine with tryptophan in the proximal SHH-3 binding domain of PTPN22, which is necessary for interaction with Csk. In vitro experiments show that the W620 variant of PTPN22 binds less efficiently to Csk, suggesting $T$ cells expressing this allele may be hyper-responsive and more prone to autoimmunity.

Objective To investigate the association of R620W polymorphism with RA in a Dutch Caucasian population and to determine whether this polymorphism associates with a qualitative phenotype of RA (remission versus progression) or a quantitative phenotype (rate of joint damage).

Methods PTPN22 genotyping was performed on DNA from 425 RA and 343 undifferentiated arthritis patients participating in the Leiden Early Arthritis Clinic and over 200 control individuals, both of Dutch Caucasian origin. Samples were genotyped using allele-specific kinetic PCR. Disease features were measured as previously published. X-rays were scored every year.

Results We confirmed association of the R620W allele of PTPN22 polymorphism with RA in our Dutch Caucasian population. Minor allele frequencies of 0.07 and 0.12 were found in controls and cases, respectively, with $P=0.008$ and an allelic odds ratio of $1.91,95 \%$ confidence interval $=1.18-3.11$. Surprisingly PTPN22 appeared to be a susceptibility gene for undifferentiated arthritis that either progresses or does not progress to RA. PTPN22 status did not influence the disease persistency, as analyzed in the remission versus non-remission group, or the disease severity as determined by analysis of joint damage. 
Figure 1

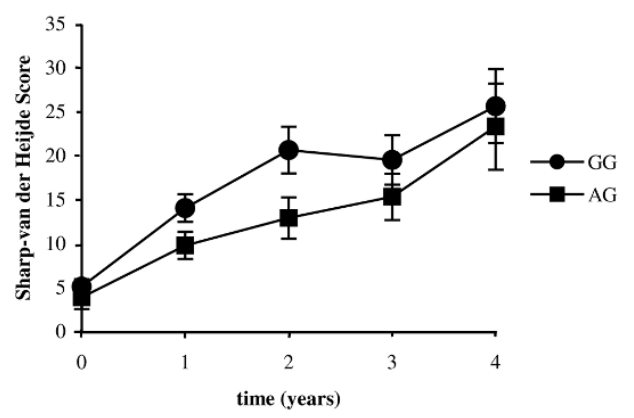

Progression of joint damage (Sharp-van der Heijde units).

Conclusion: Our results suggest that the R620W allele of PTPN22 increases susceptibility to RA but does not confer risk to a more severe disease course either with respect to joint destruction or with respect to disease severity.

\section{P99}

\section{Tryptase as a PAR-2 activator in joint inflammation} EB Kelso', L Dunning'2, JC Lockhart², WR Ferrell', R Plevin³, CP Sommerhoff ${ }^{4}$

${ }^{1}$ Centre for Rheumatic Diseases, University of Glasgow, UK; ${ }^{2}$ Biological Sciences, University of Paisley, UK; ${ }^{3}$ Department of Physiology \& Pharmacology, University of Strathclyde, Glasgow, UK; ${ }^{4}$ Department Clinical Biochemistry, University of Munich, Germany

Arthritis Res Ther 2005, 7(Suppl 1):P99 (DOI 10.1186/ar1620)

Background Protease-activated receptor-2 (PAR-2) is one of a family of Gprotein coupled transmembrane receptors activated by proteolytic release of a 'tethered' ligand. We previously reported this receptor has a pivotal role in chronic joint inflammation using a PAR-2 'knockout' mouse [1], but the serine protease responsible for its activation remains uncertain.

Objective We investigated whether $\beta$-tryptase has proinflammatory actions in the mouse knee and specifically whether a tryptase inhibitor can modulate experimentally induced chronic joint inflammation.

Methods Five micrograms of human $\beta$-tryptase was injected into the knee joint cavity of two groups of anaesthetized (Halothane $/ \mathrm{O}_{2} / \mathrm{N}_{2} \mathrm{O}$ ) mice $(30 \mathrm{~g})$ : wild-type $\left(\mathrm{PAR}^{-2^{+/+}}{ }^{\mathrm{C}} \mathrm{C} 57 \mathrm{BL} / 6 \mathrm{~J}\right.$ mice and PAR-2 gene disrupted mice (PAR-2 ${ }^{-/}$). Joint swelling was assessed by comparing caliper measurements of knee joint diameter pre-injection and post-injection. Chronic monoarthritis was induced using the same anaesthetic regime in separate wild-type mice by intra-articular and periarticular injection of Freunds complete adjuvant (FCA) (in 5\% methycellulose). In a parallel group of mice, $50 \mu \mathrm{g}$ of the tryptase inhibitor 4-amidino phenyl pyruvic acid (APPA) was co-administered with the FCA/methycellulose emulsion. Joint diameter was measured over 10 days.

Results Intra-articular injection of $\beta$-tryptase resulted in rapid joint swelling in wild-type mice that was completely abrogated in PAR-2-l- mice (Fig. 1a), suggesting that tryptase-mediated inflammatory actions require functional PAR-2. Tryptase plays an important role in mediating chronic inflammation as APPA coadministration substantially inhibited FCA-induced joint swelling (Fig. 1b). The present study extends our previous finding [1] that PAR-2 plays a key role in mediating chronic joint inflammation, by demonstrating that tryptase may be a crucial activating protease required for such PAR-2-mediated actions.

\section{Figure 1}
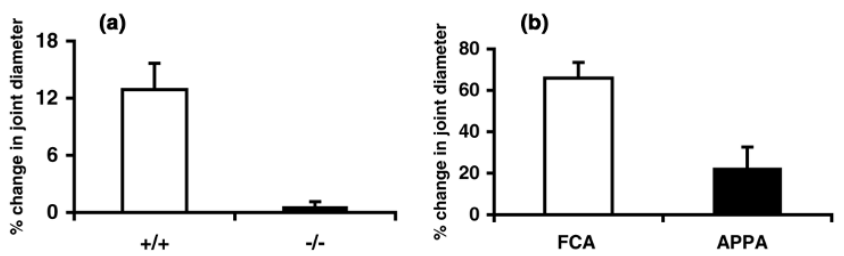

(a) Six hours after intra-articular injection of $\beta$-tryptase, knee joint swelling is evident in wild-type (PAR-2 ${ }^{+/+}$) mice but is virtually absent in PAR-2 gene disrupted (PAR$\left.2^{-l-}\right)$ mice $(P<0.001, n=4)$. (b) Knee joint swelling 10 days after Freunds complete adjuvant (FCA) treatment is significantly inhibited by co-administration of 4-amidino phenyl pyruvic acid (APPA) with FCA $(P=0.014, n=4)$.
Reference

Ferrell WR, Lockhart JC, Kelso EB, Dunning L, Plevin R, et al:: Essential role for proteinase-activated receptor-2 in arthritis. J Clin Invest 2003, 111:35-49.

\section{P100}

HLA DR-DQ haplotypes and genotypes in patients with systemic lupus erythematosus with co-existing Sjogren's syndrome, systemic lupus erythematosus without Sjogren's syndrome and primary Sjogren's syndrome: clinical and laboratory associations

Ch Georgopoulou', E Zintzaras'2, M Papadimitropoulos' ${ }^{3}$, M Spyropoulou' ${ }^{3}$, A Stavropoulou 3 , HM Moutsopoulos', MN Manoussakis'

${ }^{1}$ Department of Pathophysiology, Athens University Medical School, Athens, Greece; ${ }^{2}$ Biomathematics Unit, Thessaly University Medical School, Larissa, Greece; ${ }^{3}$ National Tissue Typing Center, George Gennimatas General Hospital, Athens, Greece

Arthritis Res Ther 2005, 7(Suppl 1):P100 (DOI 10.1186/ar1621)

Background Comparative immunogenetic studies of systemic lupus erythematosus with co-existing Sjogren's syndrome (SLE-SS), systemic lupus erythematosus without Sjogren's syndrome (SLE-noSS) and primary Sjogren's syndrome (pSS) are lacking.

Objective In the present study, we conducted a thorough evaluation of the genotype and haplotype profiles in well-defined subgroups of patients with SLE-SS, SLE-noSS and pSS, including their association with disease parameters.

Methods HLA-DRB1, HLA-DQA1 and HLA-DOB1 alleles were determined by PCR and hybridization with sequence-specific oligonucleotide probes in DNA specimens derived from 23 patients with SLE-SS, 41 patients with SLE-noSS and 55 patients with pSS (all Caucasians). Patients' records were retrospectively evaluated for various clinical and laboratory parameters.

Results Compared with healthy controls (odds ratios analyses), SLE-SS and pSS patients displayed a statistically increased frequency of the DRB $1 * 0301$ *1104 heterozygote genotype, whereas SLE-noSS patients had an increased frequency of the genotypes DQA $1{ }^{*} 0401{ }^{*} 0501$, DRB $1{ }^{*} 03011^{*} 1501$ and DQB $1{ }^{*} 0201-{ }^{*} 0602$. Such statistical associations were stronger for genotypes than single alleles. The analysis of haplotype estimated frequencies (by EM algorithm) had revealed an increased frequency of the DRB $1{ }^{*} 0301-D Q A 1 * 0501$ DQB $1{ }^{*} 0201$ haplotype, as well as of the extended haplotype DRB ${ }^{*} 0301$ -

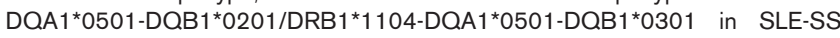
and pSS patients. In contrast, SLE-noSS patients had an increased estimated frequency of the DRB1*1501-DQA $1^{*} 0102-\mathrm{DQB} 1{ }^{*} 0602$ haplotype. In SLE-SS patients, positive associations of the DQB $1{ }^{*} 0201$ allele with anti-dsDNA (strong) and of DQA $1 * 0501$ with anti-La/SSB (marginal) were observed. In SLE-noSS, DQB $1{ }^{*} 0301-{ }^{*} 0602$ was strongly positively associated with interstitial lung disease, DQB $1{ }^{*} 0201-{ }^{*} 0602$ with central nervous system involvement, DQA $1{ }^{*} 0501$ with serositis and DRB $1{ }^{*} 1501$ with anti-dsDNA, whereas DQB1*0301 homozygosity demonstrated a significant protective effect for glomerulonephritis. In pSS patients, the DRB1*0301-*1104 and DQB1*0201${ }^{*} 0301$ genotypes were strongly positively associated with purpura, DRB $1{ }^{*} 1104$ ${ }^{*} 1601$ with arthritis, DQB $1{ }^{*} 0201-{ }^{*} 0502$ with renal tubular acidosis, DQB $1{ }^{*} 0301$ homozygosity with lymphadenopathy, DQA $1{ }^{*} 0501$ homozygosity with low $\mathrm{C} 4$ and DRB $1{ }^{*} 0301$ allele with anti-La/SSB, low C4 and cryoglobulinemia. In these patients, the extended haplotype DRB1*0301-DQA1*0501-DQB1*0201/ DRB1*1104-DQA $1 * 0501-\mathrm{DQB} 1{ }^{*} 0301$ associated strongly with the occurrence of low $\mathrm{C} 4$, anti-La/SSB and purpura.

Conclusions The present study indicates that SLE-SS and the SLE-noSS patients are immunogenetically dissimilar, whereas there is an apparent close immunogenetic relationship between SLE-SS and pSS patients. Furthermore, our data corroborate that the synergistic interactions between distinct pairs of alleles in the DR or the DQ locus confer higher relative risk for these diseases and for distinct clinical manifestations than each of these alleles individually. In pSS, the presence of the extended haplotype DRB1*0301-DQA $1{ }^{*} 0501-\mathrm{DQB} 1{ }^{*} 0201 /$ DRB $1 * 1104-D Q A 1 * 0501-D Q B 1{ }^{*} 0301$ appears to associate with adverse predictors for lymphoma development.

\section{P101}

\section{Specific inhibition of FoxO transcription factors in} rheumatoid arthritis synovial tissue

J Ludikhuize, TJM Smeets, M Vinkenoog, ME Sanders, PP Tak, KA Reedquist Division of Clinical Immunology and Rheumatology, Academic Medical Center, University of Amsterdam, The Netherlands

Arthritis Res Ther 2005, 7(Suppl 1):P101 (DOI 10.1186/ar1622)

Background Phosphorylation-dependent inactivation of FoxO transcription factors by the proto-oncogene product protein kinase B (PKB) plays a central role in promoting cellular survival, proliferation, and activation mediated by PI3kinase. PI3-kinase-dependent activation of PKB has been observed in rheumatoid 
arthritis (RA) synovial tissue, and blocking PKB activation has a protective effect in animal models of arthritis. However, the molecular mechanisms by which activation of PKB promotes arthritis have not been elucidated.

Objectives To determine whether FoxO transcription factors (FoxO1, FoxO3a, and FoxO4) are specifically inactivated in RA synovial tissue, to identify cell types in RA synovial tissue in which FoxO proteins are inactivated, and to identify inflammatory stimuli relevant to RA that inactivate FoxO transcription factors in cultured RA fibroblast-like synoviocytes (FLS) in vitro.

Methods Expression and PKB-dependent phosphorylation of FoxO1, FoxO3a, and FoxO4 were determined using specific antibodies in immunohistochemical and computer-assisted quantitative digital analysis of synovial tissue sections obtained from 12 RA and nine osteoarthritis (OA) patients. Double labelling with cell-specific antibodies was performed to identify FoxO expression and inactivation in specific cell populations. In vitro, cultured RA $(n=3)$ and OA $(n=2)$ FLS were stimulated with tumour necrosis factor (TNF) alpha, transforming growth factor (TGF) beta, or soluble CD154 prior to lysis and detection of FoxO phosphorylation by immunoblotting. FLS proliferative responses to these stimuli were determined in the presence and absence of the PI3-kinase inhibitor LY294002 by crystal violet staining.

Results Phosphorylated inactive $\mathrm{FoxO} 1$, $\mathrm{FoxO} 3$, and $\mathrm{FoxO} 4$ were observed in both RA and OA synovial tissue. Inactivation of FoxO1, FoxO3a, and FoxO4 was restricted to FLS, T lymphocytes, and synovial macrophages, respectively. Inactivation of FoxO1 and FoxO4 was significantly enhanced $(P<0.05)$ in RA synovial tissue compared with OA synovial tissue. No significant differences in the inactivation of T lymphocyte FoxO3a were observed between RA and OA synovial

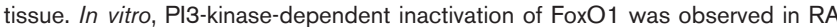
FLS following stimulation with TNF, TGF, and soluble CD154. Inactivation of FoxO1 was not sufficient to promote FLS proliferation, as only TNF and TGF but not soluble CD154 stimulated PI3-kinase-dependent FLS proliferation. Our studies demonstrate that inactivation of $\mathrm{FoxO} 1$ and $\mathrm{FoxO} 4$ is enhanced in $\mathrm{RA}$ compared with OA, provide the first (patho)physiological evidence of FoxO4 inactivation in vivo, and suggest important roles for FoxO1 and FoxO4 in maintaining, respectively, FLS and macrophage activation and survival in RA synovial tissue.

\section{P102}

\section{Quantitative and qualitative analysis of CD4+CD25+ regulatory $\mathrm{T}$ cells in systemic lupus erythematosus patients M Bonelli, JS Smolen, C Scheinecker}

Department of Rheumatology, Internal Medicine III, Medical University of Vienna, Austria

Arthritis Res Ther 2005, 7(Suppl 1):P102 (DOI 10.1186/ar1623)

$\mathrm{CD} 4{ }^{+} \mathrm{CD} 25^{+}$regulatory $\mathrm{T}$ cells (Treg) that specialize in the suppression of immune responses play a critical role in the regulation of peripheral immune tolerance and thus might be critically involved in the pathogenesis of autoimmune diseases. As for systemic lupus erythematosus (SLE), however, data concerning Treg are so far limited. We therefore initiated detailed quantitative and qualitative analysis of naturally occurring $\mathrm{CD} 4{ }^{+} \mathrm{CD} 25^{+}$Treg from SLE patients as compared with healthy controls. FACS analysis revealed increased proportions of $\mathrm{CD} 4{ }^{+} \mathrm{CD} 25^{+}$Treg, as well as activated $\mathrm{CD} 4{ }^{+} \mathrm{CD} 69^{+} \mathrm{T}$ cells, among peripheral blood mononuclear cells in SLE patients as compared with healthy controls. Ongoing experiments aim to analyze the capacity of purified CD4 ${ }^{+} \mathrm{CD} 25^{+}$Treg to suppress the proliferation of autologous or allogeneic T cells. Preliminary results indicate a tendency towards a reduced suppressive capacity of $\mathrm{CD} 4^{+} \mathrm{CD} 25^{+}$ Treg in SLE patients as compared with healthy controls. Additional experiments will determine the statistical significance of this observation and will correlate quantitative and/or qualitative defects of Tregs in SLE patients with clinical data. Finally, this study will also allow one to experimentally abrogate potential defects of Treg in SLE patients. This might provide the basis for new therapeutic concepts in the treatment of SLE and other autoimmune diseases.

\section{P103}

\section{HSP70/J-protein chaperone machines: expression analysis in granulocytes}

\section{A Zgaga-Griesz, A Adolf, S Haak, R Rzepka, I Melchers}

Clinical Research Unit for Rheumatology, University Medical Center, Freiburg, Germany

Arthritis Res Ther 2005, 7(Suppl 1):P103 (DOI 10.1186/ar1624)

Background Polymorphonuclear leucocytes (PMNLs) represent the first line of defence in innate immunity. PMNLs are the predominant cells accumulating in the synovial fluid of rheumatoid arthritis (RA) patients and play a crucial role in the development of tissue damage. Unstimulated PMNLs are postmitotic terminally differentiated cells whose default setting is death by apoptosis. Pathogens or inflammation induce a profound transcriptional and translational activity. Aberrant expression of members of the major chaperone family HSP70 occurs in synovial tissue of RA [1,2] and in PMNLs subjected to stress in the form of exercise [3] or polytrauma [4]. Activity and substrate specificity of HSP70s is regulated by the family of J-proteins. HSP7Os and J-proteins together generate important chaper- one machines, which are not only involved in classical chaperone tasks like proper protein folding but also in fine tuning of molecules involved in signal transduction and apoptotic pathways. Hardly any data exist on J-protein expression in PMNLs. Objectives Establishment of the promyeloid precursor cell line HL60 as a model for studies on the expression of several members of the HSP70/J-protein families on the level of mRNA and protein. Investigation of PMNLs from healthy donors (HD) and RA derived from peripheral blood (PB) and synovial fluid regarding the presence of members of the HSP70/J-protein chaperone machines.

Methods HL60 cells were cultivated in FCS-supplemented RPMl 1640 medium. PMNLs were separated by density gradient centrifugation followed by hypotonic lysis of erythrocytes. Total RNA was isolated and contaminating DNA was eliminated by Dnase digestion when necessary. Gene-specific primers complementary for various genes of HSP70s and J-proteins were designed and applied in RT-PCR. The presence and amount of several HSP7Os and J-proteins were analysed by western blots, applying equal amounts of protein per lane.

Results In unstimulated HL60 cells, transcripts of the constitutive member of the HSP70 family hspa8 (Hsc70) and several inducible HSP70s (hspa1b, hspa5, hspa6) could be verified. No hspa2 transcript was detected. The same expression pattern of these five genes was observed in PB-PMNLs from HD. RT-PCR revealed a clear signal for all J-proteins under analysis (Hdj2, HTid, Hsj1, Mdg1, Tpr2 and Sec63) in HL60. In contrast, we observed a complete absence of these J-protein transcripts in PB-PMNLs from HD. Interestingly, in PMNLs from RA some J-protein transcripts were expressed in PB-PMNLs or synovial fluid PMNLs. On the protein level, Hsp70, Hsc70 and BiP were detected in HL60, as well as Hdj2 and HTid. In contrast, Hsc70 and Hdj2 showed a reduced expression, and moreover BiP and $\mathrm{HTid}$ were under the level of detection in HD PB-PMNLs.

Conclusions We observed a distinct expression of several members of the HSP70/J-protein chaperone machines in PMNLs from HD or RA, and HL60. We assume that the observed reduction in protein expression of the HSP70/J-protein chaperone machines and the complete missing of J-protein transcripts contributes to the pro-apoptotic state of unstimulated PMNLs in the periphery. We suggest that the reappearance of several J-protein transcripts may be involved in the altered phenotype and prolonged survival of PMNLs in RA.

References

1. Schick C, Arbogast M, Lowka K, Rzepka R, Melchers I: Contiuous enhanced expression of the constitutive (Hsc70), but not the inducible (Hsp70) heat shock protein $\mathbf{7 0}$ in rheumatoid arthritis synovial tissue. Arthritis Rheum 2004, 50:88-93.

2. Kurzik-Dumke U, Schick C, Rzepka R, Melchers I: Overexpression of human homologs of the bacterial DnaJ chaperone in the synovial tissue of patients with rheumatoid arthritis. Arthritis Rheum 1999, 42:210-220.

3. Fehrenbach E, Passek F, Niess AM, Pohla H, Weinstock C, Dickhuth HH, Northoff H: HSP expression in human leukocytes is modulated by endurance exercise. Med Sci Sports Exerc 2000, 32:592-600.

4. Weingartmann G, Oehler R, Derkits S, Oismuller C, Fugger R, Roth E: HSP70 expression in granulocytes and lymphocytes of patients with polytrauma: comparison with plasma glutamine. Clin Nutr 1999, 18:121-124.

Acknowledgement Supported by DFG grant Me 604/6.

P104

\section{Genotype-dependent NOS-3 expression and rheumatoid} arthritis

M Cattaruzza', I Melchers ${ }^{2}$, M Hecker ${ }^{1}$

${ }^{1}$ Department of Cardiovascular Physiology, University of Göttingen, Germany; ${ }^{2}$ Clinical Research Unit for Rheumatology, University Medical Center, Freiburg, Germany Arthritis Res Ther 2005, 7(Suppl 1):P104 (DOI 10.1186/ar1625)

The ${ }^{-786} \mathrm{C}$-variant of the endothelial nitric oxide synthase nos-3 gene has been shown to be associated with coronary artery disease because of a blunted inducibility of gene expression [1]. IL-10, a cytokine involved in $\mathrm{T}_{\mathrm{H} 1} / \mathrm{T}_{\mathrm{H} 2}$-cell differentiation, is a new stimulus for NOS-3 expression [2].

We here address the question whether IL-10-induced NOS-3 expression is decreased in individuals with the ${ }^{-786} \mathrm{C} / \mathrm{C}$ genotype and, if so, whether a $\mathrm{T}_{\mathrm{H} 1}$ mediated disease like rheumatoid arthritis is associated with this genotype. Endothelial cells were isolated from an umbilical cord vein of known genotype and cultured as described [1]. The expression of NOS-3 was analysed by realtime semi-quantitative RT-PCR [1]. Genotyping was performed as described elsewhere [1]. Patients met the revised criteria of the ACR for the classification of rheumatoid arthritis, and donated blood samples after informed consent.

Primary human umbilical vein endothelial cells with the ${ }^{-786} \mathrm{C} / \mathrm{C}$ genotype did not respond with an increase in NOS-3 expression to $\mathrm{IL}-10$ incubation $(5 \mathrm{ng} / \mathrm{ml})$. This defect could be repaired after pre-incubation of the cells with a decoy oligonucleotide $(10 \mu \mathrm{mol} / \mathrm{l})$ directed against the C-variant of the promoter. Among 587 patients with rheumatoid arthritis tested, incidences for the ${ }^{-786} \mathrm{C} / \mathrm{C}$ genotype were significantly higher than in the general population $(17 \%$ versus $11.7 \% ; P<0.01)$. NOS-3 is one mediator of anti-inflammatory IL-10 actions. Individuals with the ${ }^{-786} \mathrm{C} / \mathrm{C}$ nos-3-genotype have an increased risk for the development of rheumatoid arthritis. This might be due to the IL-10 insensitivity of the C-variant of the promoter. 
References

1. Cattaruzza M, Guzik TJ, Slodowsky W, Pelvan A, Becker J, Halle M, Buchwald $A B$, Channon KM, Hecker, M: Shear stress insensitivity of endothelial nitric oxide synthase expression as a genetic risk factor for coronary heart disease. Circ Res 2004, 95:841-847.

2. Cattaruzza $M, S^{3}$ odowski $W$, Stojakovic $M$, Krzesz R, Hecker M: Interleukin-10 induction of nitric-oxide synthase expression attenuates CD40-mediated interleukin-12 synthesis in human endothelial cells. $J$ Biol Chem 2003, 278:37874-37880.

Acknowledgement Supported by a grant of the BMBF (competence network rheumatism) to IM.

\section{P105}

\section{Anti-high-density lipoprotein antibodies and lipoprotein} characteristics in systemic lupus erythematosus-related cardiovascular disease

\section{J Su, A Cederholm, E Hurt-Camejo, E Svenungsson, J Frostegård}

Department of Medicine, Karolinska University Hospital, Stockholm, Sweden

Arthritis Res Ther 2005, 7(Suppl 1):P105 (DOI 10.1186/ar1626)

Background The risk of cardiovascular disease (CVD) in systemic lupus erythematosus (SLE) is exceedingly high. Previously, a lupus pattern of dyslipidemia has been described (raised triglycerides, and low high-density lipoprotein [HDL]) and we recently reported that these lipid abnormalities were associated with tumor necrosis factor (TNF) activity.

Methods Twenty-three women (52 \pm 8.2 years) with SLE and a history of CVD (SLE cases) were compared with 26 age-matched women with SLE and no clinical manifestations of CVD (SLE controls) and 26 age-matched healthy women (controls). Lipoprotein-related measurements included lipoprotein particle size determination by use of NMR spectroscopy and affinity of low-density lipoprotein (LDL) to proteoglycans. Antibodies against apolipoprotein A1 (apoA1) in HDL and TNF was determined by ELISA. Common carotid intima-media thickness was measured by B-mode ultrasound as a surrogate measure of atherosclerosis.

Results Anti-apoA1 were raised in SLE cases as compared with SLE controls $(P=0.03)$ and controls $(P=0.001)$, and in SLE controls as compared with controls $(P=0.01)$. Among SLE cases, anti-apoA1 was associated with TNF $(P=0.01)$. Small dense LDL were more common among SLE controls and controls than in SLE cases $(P=0.036$ and 0.086 , respectively). Small HDL was more common among controls than in SLE cases and SLE controls $(P=0.001)$. LDL association with proteoglycans did not differ between groups Activity in the TNF system was significantly associated with triglycerides and negatively with $\mathrm{HDL}(P<0.01)$.

Conclusion SLE-related dyslipidemia showed a surprising pattern with large LDL and HDL rather than small; that is, not an expected 'atherogenic' lipid profile. Anti-apoA1 antibodies where strongly associated with CVD in SLE. Whether they may play a pathogenic role (e.g. by inhibiting anti-inflammatory properties of $\mathrm{HDL}$ ) is presently under investigation.

Acknowledgements Supported by the Swedish Heart Lung Foundation, the King Gustav V 80th Birthday Fund, the Torsten and Ragnar Söderberg Foundation, and the Swedish Science Fund.

\section{P106}

Discrepancy between jun/fos proto-oncogene mRNA and protein expression in the rheumatoid arthritis synovial membrane

\section{R Huber', B Stuhlmüller², E Kunisch', RW Kinne'}

${ }^{1}$ Experimental Rheumatology Unit, Friedrich Schiller University Jena, Germany; ${ }^{2}$ Department of Rheumatology and Clinical Immunology, Charité University Hospital, Humboldt University of Berlin, Germany

Arthritis Res Ther 2005, 7(Suppl 1):P106 (DOI 10.1186/ar1627)

Protein/mRNA expression of cjun, junB, junD, and cfos, levels of the mRNA-destabilizing proteins AUF-1 and tristetraprolin (TTP), and levels of the mRNA-stabilizing protein HuR were assessed in synovial membranes (SM) from patients with rheumatoid arthritis (RA), osteoarthritis (OA), joint trauma (JT), or postmortem normal controls (NC) using ELISA/western blotting and real-time RT-PCR.

Protein expression for Jun/Fos proto-oncogenes was significantly increased (JunB and JunD) or numerically increased (cJun and cFos) in RA SM compared with OA, JT, and NC. In contrast, jun/fos mRNA expression was significantly decreased (cjun and junB) or numerically decreased (junD and cfos) in RA and OA compared with JT or NC.

Protein expression levels of AUF-1 were comparable among the different groups. For TTP and HuR, interestingly, significantly increased protein expression was observed in RA and OA SM compared with either JT or NC.

Discrepancies between the mRNA and protein expression for several jun/fos genes suggest broad alterations of post-transcriptional processes in the RA SM. Whereas increased levels of mRNA-destabilizing TTP may contribute to the low levels of jun/fos mRNA, abundant mRNA-stabilizing HuR may augment translation of the remaining mRNA into protein. As a consequence, both increased expression of AP-1-dependent target genes by the activating transcription factors cJun/cFos and modified binding activity of the resulting AP-1 complexes via the
Acknowledgements This work was supported by grants from the Interdisciplinary Center of Clinical Research Jena (including a grant for junior researchers to RH; FKZ 01ZZ9602/FKZ 01ZZ0105) and the Jena Centre for Bioinformatics (FKZ 0312704B). RH was also supported by a grant of the German National Academic Foundation.

\section{P107}

Distinct biological properties of human mesenchymal stem cells from different sources

C De Bari', F Dell'Accio', J Vanlauwe'2, C Pitzalis', FP Luyten²

${ }^{1}$ Rheumatology Department, King's College London, UK; ${ }^{2}$ Rheumatology

Department, University Hospitals KU Leuven, Belgium

Arthritis Res Ther 2005, 7(Suppl 1):P107 (DOI 10.1186/ar1628)

Background Mesenchymal stem cells (MSCs) have been isolated from different tissues/organs, but it is not clear whether they possess distinct biological properties. We have previously characterized MSCs from the adult human synovial membrane (SM), which can differentiate at the single cell level to cartilage, bone, adipocytes, and skeletal muscle [1-3]. We have also reported that cells isolated from the adult human periosteum $(P)$ are chondrogenic in vitro [4]. In the present work, we show that expanded periosteal cells are multipotential. We then compare the chondrogenic and osteogenic potentials of P-MSCs with those of SM-MSCs.

Methods MSC populations were enzymatically released from the SM and periosteum of four adult human donors. To test multipotency, P-MSCs were subjected to in vitro differentiation assays or injected into regenerating tibialis anterior muscles of nude mice. In vitro chondrogenesis was tested in micromass culture in the presence of transforming growth factor beta in a chemically defined medium and assessed by histochemistry for cartilage proteoglycans and by quantitative RT-PCR for chondrocyte markers. To investigate bone formation in vivo, MSCs were seeded into Collagraft scaffolds and implanted under the skin of nude mice. Bone formation was assessed by histology and the human origin investigated by in situ hybridization for human $A L U$ genomic repeats and by RTPCR for bone markers using primers specific for human cDNA.

Results P-MSCs underwent chondrogenesis, osteogenesis, and adipogenesis in vitro as well as myogenesis in vivo. Multipotency was inherent at the single cell level. P-MSCs were compared with SM-MSCs from the same donors in the capacity to form cartilage in vitro and bone in vivo. Under our experimental conditions, SM-MSCs displayed greater chondrogenic potential than P-MSCs with higher contents of cartilage-specific proteoglycans and higher expression levels of mature chondrocyte markers. For bone formation, engraftment of P-MSCs and SM-MSCs into Collagraft was comparable and either MSC population survived long term in vivo (20 weeks). Histologically, no bone was evident at 4 weeks. At later time points (8-20 weeks), abundant bone formation was detected consistently in all periosteal samples. In contrast, bone was rarely observed, and in small amounts, in the synovial samples, with most human cells contributing to a fibrouslike tissue. In all cases, bone was mostly of human origin. As evaluated by quantitative RT-PCR, the expression levels of human OC, normalized for human beta-actin, were significantly higher in the periosteal samples than in the synovial ones. Bone was neither retrieved in empty Collagraft scaffolds nor in Collagraft scaffolds seeded with human dermal fibroblasts used for a cell negative control. Conclusions Expanded P-MSCs can differentiate to cartilage, bone, adipocytes, and skeletal muscle. Importantly, SM-MSCs displayed a greater chondrogenic potential in vitro than P-MSCs. By contrast, P-MSCs formed bone in vivo consistently and reproducibly as opposed to SM-MSCs. Our results suggest that MSCs derived from different tissue have distinct biological properties, thereby pointing to a need for development of quality controls for MSC preparations in clinical settings.

References

1. De Bari C, Dell'Accio F, Tylzanowski P, Luyten FP: Multipotent mesenchymal stem cells from adult human synovial membrane. Arthritis Rheum 2001, 44:1928-1942.

2. De Bari C, Dell'Accio F, Vandenabeele F, Vermeesch JR, Raymackers JM, Luyten FP: Skeletal muscle repair by adult human mesenchymal stem cells from synovial membrane. J Cell Bio/ 2003, 160:909-918.

3. De Bari C, Dell'Accio F, Luyten FP: Failure of in vitro-differentiated mesenchymal stem cells from the synovial membrane to form ectopic stable cartilage in vivo. Arthritis Rheum 2004, 50:142-150.

4. De Bari C, Dell'Accio F, Luyten FP: Human periosteum-derived cells maintain phenotypic stability and chondrogenic potential throughout expansion regardless of donor age. Arthritis Rheum 2001, 44:85-95.

\section{P108}

\section{Regulation of T-cell differentiation by IL-4R $\alpha$-chain single} nucleotide polymorphisms

A Skapenko, I Prots, S Mattyasovszky, CL Yoné, JR Kalden, H Schulze-Koops

Nikolaus Fiebiger Center for Molecular Medicine, Clinical Research Group III, University of Erlangen-Nuremberg, Erlangen, Germany

Arthritis Res Ther 2005, 7(Suppl 1):P108 (DOI 10.1186/ar1629)

Chronic inflammation in rheumatoid arthritis (RA) is mediated by repeatedly activated proinflammatory Th1 cells. In contrast, Th2 cells that might downmodulate the 
chronic autoimmune response are rarely found in RA. It has been previously documented that RA T cells are severely impaired in their ability to differentiate into Th2 effectors while exerting enhanced Th1 differentiation. The mechanisms underlying this functional abnormality, however, have not been delineated. As IL-4 is a most critical determinant in regulating immune responses by promoting Th2 cell development and inhibiting Th1 cell differentiation, we analyzed the role of single nucleotide polymorphisms (SNPs) in the IL-4 receptor (IL-4R) $\alpha$-chain, which is critical for binding of IL-4 and for IL-4 signal transduction, in the differentiation of human T cells. Three hundred and sixty-one healthy individuals were genotyped by allele-specific PCR for the two IL-4R $\alpha$-chain SNPs that are located in functionally important regions of the IL-4R $\alpha$-chain - the I50V SNP50 and the O551R SNP551 in the IL4-binding and STAT6-binding domains, respectively. Naive and memory CD4-positive $T$ cells were isolated from the peripheral blood of individuals who were homozygous for either allele at SNP50 and SNP551, and were primed for 5 days with mAbs to CD28 and/or CD3 in the presence or absence of exogenous IL-4. The phenotype of the resulting differentiated effector cells was then analyzed by flow cytometric analysis of cytoplasmic cytokines. The SNP551 alleles did not affect T-cell differentiation. In contrast, the inhibitory effect of IL-4 on Th1 cell differentiation was significantly diminished in CD4 T cells that were homozygous for the mutated allele at SNP50 (50V) as compared with those with the wild type allele (150). Likewise, the augmenting effect of IL-4 on Th2 cell differentiation was enhanced on T cells that were homozygous for the wild-type allele as compared with $T$ cells expressing the mutant allele. These data indicate that the mutant allele of the IL-4R $\alpha$-chain at SNP50 is associated with a decreased T-cell response to IL4. To delineate a potential mechanism of different responses to IL-4 in the cells expressing different alleles of the IL-4R, T cells form individuals who were homozygous for either the wild-type or the mutant allele at SNP50 were primed with different concentrations of IL-4 and analyzed by flow cytometry for STAT6 and phosphorylated STAT6. Whereas STAT6 concentrations were not different between T-cell expressing 150 or V50, STAT6 phosphorylation in response to IL-4 stimulation was significantly reduced in T cells expressing the V50 allele compared with T cells expressing 150. Thus, the V50 SNP50 allele of the IL-4R $\alpha$-chain might regulate T-cell differentiation by diminishing T-cell responses to IL-4, resulting in reduced STAT- 6 phosphorylation and subsequently in diminished Th2 cell differentiation. The V50 SNP50 allele might thereby contribute to the development of unbalanced Th subset activation, as characteristic for autoimmune diseases, such as RA. Acknowledgements Supported by the Deutsche Forschungsgemeinschaft, the Interdisciplinary Center for Clinical Research at the University of Erlangen-Nuremberg, and the Dr Robert Pfleger Foundation.

\section{P109}

\section{Role of IL-10 as a susceptibility factor for rheumatoid} arthritis and cardiovascular disease

\section{FAS Kurreeman', J De Vries', R Westendorp², AH Van Den Biggelaar ${ }^{2}$,} JJM Schonkeren', REM Toes', TWJ Huizinga'

${ }^{1}$ Department of Rheumatology, Leiden University Medical Centre, Leiden, The Netherlands; ${ }^{2}$ Department of General Internal Medicine, Section Gerontology and Geriatrics, Leiden University Medical Centre, Leiden, The Netherlands Arthritis Res Ther 2005, 7(Suppl 1):P109 (DOI 10.1186/ar1630)

IL-10 is both an immunoinhibitory (monocytes and T cells) and an immunostimulatory (B cells) cytokine that may confer susceptibility and modulate disease expression in rheumatoid arthritis (RA). The capacity to produce IL-10 (as measured by production of IL-10 in a lipopolysaccharide-stimulated whole blood culture) differs about fourfold between individuals. By comparing variance between monozygotic and dizygotic twins it was estimated that $60 \%$ of these differences are caused by genetic factors.

To determine relevance for susceptibility to diseases, we determined production levels of IL-10 in lipopolysaccharide-induced whole blood cultures from 76 new incident, non-treated RA patients and 63 healthy controls. RA patients had a $50 \%$ lower IL-10 production than controls $(P<0.001)$. In a cohort of women ( $\geq 85$ years) with high risk of cardiovascular death [1], we observed that low innate IL-10 production yielded a threefold increase in risk of dying, which, interestingly, correlates with the presence of the A allele of the IL-10 -2849 promoter polymorphism $(P=0.021)$.

To elucidate this genetic basis of IL-10 secretion and the functionality of IL-10 promoter polymorphisms, we used the technique of allele-specific transcript quantification to characterise the ratio between two alleles of the IL-10 gene in 15 healthy heterozygous individuals. We identified two groups whereby five healthy donors exhibited a $1: 1$ ratio whereas seven exhibited a ratio $>1$ $(P<0.0017)$ [2]. Donors heterozygous for haplotype IL-10.2 were only prevalent in the group with higher allelic expression ratios. The G allele of the IL-10 promoter single nucleotide polymorphism -2849 tags this haplotype, providing functional evidence that differential allelic transcription partly explains the constant associations found with IL-10 production levels.

In conclusion, this study provides evidence that IL-10 haplotypes dictating production of IL-10 are involved not only in conferring susceptibility to RA, but also in risk for cardiovascular death in old age.
References

1. Van Den Biggelaar AH, De Craen AJ, Gussekloo J, Huizinga TW, Heijmans BT, Frolich M, Kirkwood TB, Westendorp RG: Inflammation underlying cardiovascular mortality is a late consequence of evolutionary programming. FASEB J 2004, 18:1022-1024.

2. Kurreeman FA, Schonkeren JJ, Heijmans BT, Toes RE, Huizinga TW: Transcription of the IL10 gene reveals allele-specific regulation at the mRNA level. Hum Mol Genet 2004, 13:1755-1762.

\section{P110}

\section{Regulation of myeloid cell function and MHC class II} expression by tumor necrosis factor

\section{RB Mueller,2, A Skapenko',2, M Grunke ${ }^{2}$, J Wendler ${ }^{3}$, B Stuhlmuller 4 ,} JR Kalden ${ }^{2}$, H Schulze-Koops ${ }^{1,2}$

${ }^{1}$ Nikolaus Fiebiger Center for Molecular Medicine, Clinical Research Group III, University of Erlangen-Nuremberg, Erlangen, Germany; ${ }^{2}$ Department of Internal Medicine III and Institute for Clinical Immunology, University of Erlangen-Nuremberg, Erlangen, Germany; ${ }^{3}$ Rheumatologische Gemeinschaftspraxis, Erlangen, Germany; ${ }^{4}$ Department of Rheumatology and Clinical Immunology, Charité University Hospital, Berlin, Germany Arthritis Res Ther 2005, 7(Suppl 1):P110 (DOI 10.1186/ar1631)

Neutralizing agents to tumor necrosis factor (TNF) are the most successful means to ameliorate systemic autoimmune inflammation. Neutralization of TNF, however, is often associated with the development of autoantibodies, in particular to nuclear antigens, the mechanisms of which are unknown. Here, we analyzed the effect of TNF and its neutralization on MHC class II expression and function of antigen-presenting myeloid cells in rheumatoid arthritis (RA). Monocytes were isolated from the peripheral blood of RA patients before and after anti-TNF mAb treatment and from controls by negative selection, differentiated in vitro into macrophages and analyzed by flow cytometry for HLA-DR expression. T-cell responses to activation by myeloid cells were assessed in proliferation assays, and mRNA levels of the class II transactivator (CIITA) were determined by semiquantitative RT-PCR. HLA-DR expression was significantly reduced on myeloid cells from RA patients with active disease, but was increased to normal levels after TNF mAb treatment. Concordantly, in vitro application of TNF to monocytes from healthy individuals reduced their ability to upregulate HLA-DR during differentiation to macrophages and, importantly, inhibited their ability to stimulate $T$ cells in mixed lymphocyte reactions. Molecular analysis revealed that the effect of TNF on HLA-DR expression was mediated via suppression of the transcription factor CIITA. The data indicate that TNF decreases HLA-DR expression by reducing CIITA mRNA levels in myeloid cells, functionally resulting in a decreased stimulatory capacity of myeloid cells for T cells. Concordantly, ameliorating disease activity in chronic inflammatory diseases by neutralizing TNF restores HLA-DR expression of myeloid cells and their ability to stimulate T cells. Thus, anti-TNF treatment might lead to augmented T-cell activation by myeloid cells, thereby promoting immune responses to (auto)antigens and the development of anti-nuclear antibodies that are frequently associated with anti-TNF therapy.

Acknowledgements Supported by the Deutsche Forschungsgemeinschaft, the German Ministry for Education and Research, and the Interdisciplinary Center for Clinical Research at the University Hospital of the University of Erlangen-Nuremberg.

\section{P111}

Specific contributions of H-Ras and N-Ras GTPases to T-lymphocyte oxidative stress and integrin-dependent retention in rheumatoid arthritis

\section{PHJ Remans, ME Sanders, PP Tak, KA Reedquist}

Division of Clinical Immunology and Rheumatology, Academic Medical Center, University of Amsterdam, The Netherlands

Arthritis Res Ther 2005, 7(Suppl 1):P111 (DOI 10.1186/ar1632)

Background In rheumatoid arthritis (RA), constitutive activation of integrins promotes retention of oxidatively stressed, activated T lymphocytes in synovial tissue where they perpetuate inflammation through cell-cell contact with synovial stromal cells, macrophages and plasma cells. We have previously demonstrated that activation of Ras GTPases, coupled with inactivation of the related GTPase Rap1, is responsible for oxidative stress in RA synovial fluid (SF) T lymphocytes [1]. Inactivation of Rap1, however, is incongruous with the observation that Rap1 signaling is absolutely required for integrin function in T lymphocytes. Because T lymphocytes express multiple Ras homologs (H-Ras, K-Ras, and N-Ras), we explored the possibility that these proteins may make distinct contributions to reactive oxygen species (ROS) regulation and integrin function in T lymphocytes. Objectives To determine which Ras homologs are activated in RA SF T lymphocytes, to determine whether Ras homologs selectively regulate ROS production and integrin-dependent adhesion in T lymphocytes, and to use pharmacological inhibitors to characterize downstream signaling pathways by which Ras homologs may mediate these effects. 
Methods Activation of H-Ras, K-Ras, and N-Ras homologs in RA SF T lymphocytes were determined by activation-specific GTPase precipitation and immunoblot analysis. T-lymphocyte intracellular ROS production was measured by FACS-based analysis of DCFA oxidation. Control, active Ras, and RapGAP constructs were introduced into human Jurkat $T$ cells and healthy donor peripheral blood $\mathrm{T}$ lymphocytes by electroporation and nucleofection techniques, respectively. For ROS analysis, cells were cotransfected with RFP to detect transfected cells. For static adhesion assays, a luciferase reporter plasmid was used to detect transfected cells: cells were allowed to adhere to immobilized fibronectin or recombinant human intracellular adhesion molecule, non-adherant cells washed off, and adherant cells quantitated by luciferase assays. In some experiments, cells were preincubated with the pharmacological inhibitors BAPTAAM, LY294002, catalase, and DPI prior to ROS or adhesion analysis.

Results All three homologs of Ras were found to be constitutively activated in RA SF T lymphocytes. Active H-Ras, but not K-Ras or N-Ras, stimulated ROS production in both Jurkat and peripheral blood T lymphocytes. H-Ras-induced ROS production was sensitive to BAPTA-AM and catalase, but not LY294002 or DPI, indicating a calcium-sensitive hydrogen peroxide source of ROS that does not require a functional NADPH oxidase. These results are consistent with our previous observations in purified RA SF T lymphocytes. In contrast, N-Ras, but not H-Ras or $\mathrm{K}$-Ras, induced integrin-dependent adhesion in Jurkat $\mathrm{T}$ cells and peripheral blood $\mathrm{T}$ lymphocytes. N-Ras-induced adhesion was sensitive to LY294002, indicative of PI3-kinase signaling. Additionally, inactivation of Rap1 with RapGAP had no effect on N-Ras-induced adhesion. This represents the first (patho)physiological signaling pathway by which integrins can be activated independently of Rap1. Our results suggest that selective targeting of $\mathrm{H}$-Ras and $\mathrm{N}$-Ras may allow specific modulation of T-cell ROS production and integrin activation in RA T lymphocytes.

Reference

1. Remans PHJ, Gringhuis SI, van Laar JM, Sanders ME, Papendrect-van der Voort EAM, Zwartkruis FJT, Nivine Levarht EW, Rosas M, Coffer PJ, Breedveld FC, et al.: Rap1 signaling is required for suppression of Ras-generated reactive oxygen species and protection against oxidative stress in T lymphocytes. J Immuno/ 2004, 173:920-931.

\section{P112}

Requirement of IL-17 receptor signaling in resident synoviocytes for development of full blown destructive arthritis E Lubberts ${ }^{1,2}$, P Schwarzenberger ${ }^{2}$, W Huang ${ }^{2}$, JR Schurr ${ }^{2}$, JJ Peschon ${ }^{3}$, WB van den Berg', JK Kolls ${ }^{2}$

${ }^{1}$ University Medical Center St Radboud, Department of Rheumatology, Nijmegen, The Netherlands; ${ }^{2}$ LSU Health Sciences Center, Department of Medicine, Gene Therapy Program, New Orleans, Louisiana, USA; ${ }^{3}$ Amgen, Seattle, Washington, USA

Arthritis Res Ther 2005, 7(Suppl 1):P112 (DOI 10.1186/ar1633)

Chronic arthritis is characterized by persistent joint inflammation and concomitant joint destruction. IL-17 is a novel proinflammatory T-cell cytokine suspected to be involved in inflammatory and autoimmune diseases such as rheumatoid arthritis. Here, we report that IL-17 receptor (IL-17R) signaling is required in resident synovial cells for full progression of chronic synovitis and bone erosion. Repeated injections of Gram-positive bacterial cell wall fragments (SCW) directly into the knee joint of naive IL-17R-deficient (IL-17 $\mathrm{R}^{-/-}$) mice had no effect on the acute phase of arthritis but prevented progression to chronic destructive synovitis as was noted in wild-type (wt) mice. Micro-array analysis revealed significant downregulation of leukocyte-specific chemokines, selectins, collagenase-3, and IL-1 in the synovium of IL-17R-1- mice. Bone marrow (BM) chimeric mice revealed the need for IL-17/L-17R signaling in resident synovial cells for development of full blown synovitis. Chimeric mice of host wt and donor IL-17R $\mathrm{R}^{-/-} \mathrm{BM}$ cells developed destructive synovitis in this chronic relapsing SCW arthritis model similar to wt $\rightarrow$ wt chimeras. In contrast, chimeric mice of host IL-17R $\mathrm{R}^{-/-}$and donor wt BM cells were protected from full blown destructive arthritis similar to IL-17R $\mathrm{R}^{-1-} \rightarrow$ $\mathrm{IL}-17 \mathrm{R}^{-/-}$chimeras. These data strongly suggest $\mathrm{T}$-cell IL-17-IL-17R signaling in resident synovial cells to be a pivotal mechanism through which an acute macrophage-driven joint inflammation progresses into a chronic destructive synovitis. Prevention of local synovial IL-17-IL-17R signaling warrants consideration as a therapeutic target in chronic destructive arthritis.

\section{P113}

\section{Different molecules at the surface of stimulated $T$ cells induce IL-1beta, tumour necrosis factor and IL-1RA in human monocytes}

D Burger, N Molnarfi, L Gruaz, JM Dayer

Division of Immunology and Allergy, Clinical Immunology Unit, Faculty of Medicine, University Hospital, Geneva, Switzerland

Arthritis Res Ther 2005, 7(Suppl 1):P113 (DOI 10.1186/ar1634)

Imbalance in cytokine homeostasis is thought to play an important part in the demonstrated that T cells might exert a pathological effect through direct cellular contact with monocyte-macrophages, inducing a massive upregulation of IL-1 $\beta$ and tumour necrosis factor (TNF) [1]. We showed that this mechanism that might be relevant to chronic inflammation is specifically inhibited by high-density lipoproteins (HDL) [2]. Like many other stimuli, besides proinflammatory cytokines, the contact-mediated activation of monocytes induces the production of cytokine inhibitors such as IL-1Ra. HDL inhibited the production of IL-1 $\beta$ and TNF but not that of IL-1Ra induced in monocytes activated by membranes isolated from stimulated T cells to mimic cellular contact. This was also the case in peripheral blood mononuclear cells stimulated by either phytoheamagglutinin or tetanus toxoid. Similarly, IL-1Ra mRNA expression was not inhibited contrary to IL-1 $\beta$ and TNF mRNA. This demonstrates that different molecules at the surface of stimulated HUT-78 cells are involved in the induction of IL-1 1 , TNF and IL-1Ra in monocytes, IL-1 $\beta$ and TNF being activated by HDL-specific ligand(s). Separation of CHAPS-solubilized membrane molecules by liquid isoelectric focusing showed that two activity peaks were present; one activating IL-1 $\beta$, TNF and IL-1Ra production, the other inducing the production of IL-1Ra in the absence of IL-1 $\beta$ and TNF. Further isolation of these two types of factor by gel filtration demonstrated that factor(s) inducing IL-1 $\beta$, TNF and IL-1Ra displayed a $M_{r}$ around $40,000 \mathrm{kDa}$, whereas factors inducing IL-1Ra only displayed $M_{r}$ around $70,000 \mathrm{kDa}$ and $30,000 \mathrm{kDa}$. Thus different factors are expressed at the surface of stimulated $\mathrm{T}$ cells that differentially trigger the production of proinflammatory and anti-inflammatory factors, and are differently affected by HDL.

References

1. Burger D, Roux-Lombard P, Chizzolini C, Dayer JM: Cell-cell contact in chronic inflammation: the importance to cytokine regulation in tissue destruction and repair. In Cytokines and Joint Injury. Edited by van den Berg WB, Miossec P. Basel, Birkhäuser Verlag; 2004:165-188. [Parnham MJ (Series Editor): Progress in Inflammation Research.]

2. Hyka N, Dayer JM, Modoux C, Kohno T, Edwards CK, III, Roux-Lombard P, Burger D: Apolipoprotein A-I inhibits the production of interleukin1 beta and tumor necrosis factor-alpha by blocking contact-mediated activation of monocytes by T lymphocytes. Blood 2001, 97:2381-2389.

\section{P114}

\section{Infliximab treatment does not induce apoptosis in} peripheral blood mononuclear cells up to 24 hours after initiation of treatment in rheumatoid arthritis patients CA Wijbrandts, P Reinders-Blankert, P Klarenbeek, TJM Smeets, MJ Vervoordeldonk, PP Tak

Clinical Immunology and Rheumatology, Academic Medical Center/University of Amsterdam, The Netherlands Arthritis Res Ther 2005, 7(Suppl 1):P114 (DOI 10.1186/ar1635)

Background Apoptosis of peripheral blood $\mathrm{T}$ lymphocytes from patients with Crohn's disease has been described after in vitro activation followed by incubation with infliximab. These ex vivo data raised the question of whether in vivo treatment of rheumatoid arthritis (RA) with the chimeric tumor necrosis factor alpha antibody, infliximab, causes apoptosis in peripheral blood mononuclear cells. This study was designed to detect the early effects of infliximab treatment on apoptosis in the peripheral blood of patients with RA.

Methods Ten patients with active RA (Disease Activity Score [DAS 28] > 3.2) received $3 \mathrm{mg} / \mathrm{kg}$ infliximab intravenously in combination with methotrexate (mean dose of $25 \mathrm{mg}$ weekly). All 10 patients underwent blood sampling before, 1 hour after and 24 hours after the administration of infliximab. Apoptosis was determined using double staining with annexin- $\mathrm{V}$, as an early marker of apoptosis, and 7-amino-actinomycin D (AAD) to exclude necrotic cells from this population. Peripheral blood erythrocytes were lysed and the mononuclear cells were incubated with the different antibodies. The percentages of annexin-V-positive and 7AAD-negative monocytes and lymphocytes were analyzed by flow cytometry. For statistical analysis, a paired $t$ test was used to compare the percentages of apoptotic cells 1 hour and 24 hours after treatment with those at baseline.

Results All samples were analyzed for the presence of and change in apoptosis. At baseline, the median percentage of apoptotic monocytes was $0.90 \%$ (range $6.23-0.19$ ); 1 hour after treatment, the median percentage was $0.56 \%$ (range 2.90-0.32) and 24 hours after treatment the median percentage was $0.21 \%$ (range 2.90-0.00). In the lymphocyte population, the median percentage of apoptotic cells was $0.98 \%$ (range $2.15-0.00$ ) at baseline as compared with $0.52 \%$ (range 6.30-0.00) 1 hour after treatment and $0.39 \%$ (range 2.85-0.00) 24 hours after infliximab administration.

Conclusion In this in vivo study we found no statistically significant increase from baseline in the percentage of apoptotic monocytes or lymphocytes in the peripheral blood of RA patients at 1 hour or $\mathbf{2 4}$ hours after infliximab treatment. Acknowledgement Supported by Centocor. 


\section{P115}

Resistance of rheumatoid arthritis synovial fibroblasts to p38 MAP-kinase inhibition of pro-destructive functions mediated by tumor necrosis factor alpha/tumor necrosis factor receptor-1

E Kunisch', B Ukena1, R Fuhrmann' ${ }^{\text {, A Roth }}{ }^{2}$, R Winter ${ }^{2}$, RW Kinne1

${ }^{1}$ Experimental Rheumatology Unit, Friedrich Schiller University Jena, Germany; ${ }^{2}$ Clinic of Orthopedics, Friedrich Schiller University Jena, Germany

Arthritis Res Ther 2005, 7(Suppl 1):P115 (DOI 10.1186/ar1636)

Objectives In rheumatoid arthritis (RA), tumor necrosis factor (TNF) alpha is a major inductor of the proinflammatory/pro-destructive functions of synovial fibroblasts (SFB). These effects are predominantly mediated via the TNF receptor-1 (TNFR1). In addition to the NF-KB pathway, the p38 MAP kinase seems to play a central role for the underlying signal transduction. In the present study, RA-SFB were compared with osteoarthritis (OA)-SFB concerning the TNF- $\alpha / T N F R 1 / 2$ induced secretion of IL-6, IL-8, prostaglandin $\mathrm{E}_{2}\left(\mathrm{PGE}_{2}\right)$, and matrix metalloproteinase-1/tissue inhibitor of matrix metalloproteinase-1 (MMP-1/TIMP-1), as well as the sensitivity to p38 MAP-kinase inhibition.

Methods Early-passage (second) RA-SFB and OA-SFB were analyzed for TNFR expression by FACS. The cells were then stimulated with TNF- $\alpha(10 \mathrm{ng} / \mathrm{ml})$ or agonistic anti-TNFR1 (HTR-9) or anti-TNFR2 monoclonal antibodies (UTR-1; $10 \mu \mathrm{g} / \mathrm{ml}$ each) with/without inhibition of the p38 kinase by SB203580 $(1 \mu \mathrm{M})$. Secretion of IL-6, IL-8, PGE 2 , MMP-1, and TIMP-1 was analyzed by ELISA.

Results RA-SFB and OA-SFB both expressed TNFR1 and TNFR2 on their surface, without significant differences between the two groups. Secretion of IL-6, IL-8, PGE 2 , and MMP-1, but not TIMP-1, was significantly augmented by

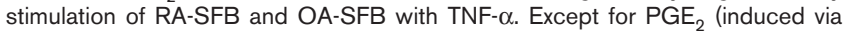
both TNFRs), these effects were exclusively mediated via the TNFR1. Inhibition of p38 kinase reduced the secretion of IL- 6 and $P G E_{2}$ significantly and equally well in RA-SFB and OA-SFB. However, the secretion of MMP-1 was significantly suppressed only in OA-SFB, whereas RA-SFB were insensitive to the inhibition of MMP-1 secretion by $\mathrm{p} 38$ inhibition.

Conclusion In early-passage RA-SFB and OA-SFB, TNF- $\alpha$-induced proinflammatory/pro-destructive functions are predominantly mediated by TNFR1. Strikingly, RA-SFB are partially resistant to the suppression of pro-destructive MMP-1 by p38 MAP-kinase inhibition. The underlying structural or functional alterations of the p38 MAP kinase in RA-SFB may contribute to the pathogenesis and/or therapeutic sensitivity of RA.

Acknowledgements This study was supported by the German Federal Ministry of Education and Research (BMBF; grant FKZ 01 ZZ0105 to RWK, Interdisciplinary Center for Clinical Research Jena) and the German Research Foundation (DFG; grant $\mathrm{KI} 439 / 7-1$ to RWK), as well as a grant for the advancement of female scientists to EK (LUBOM Thuringia).

\section{P116}

Patterns of gene expression in rheumatoid arthritis synovial tissue: inter-patient variability greater then intra-patient variability in a small study

J Lindberg', E af Klint², A Stark ${ }^{3}$, T Andersson², P Nilsson², L Klareskog², A Ulfgren ${ }^{2}$, J Lundeberg ${ }^{1}$

${ }^{1}$ Royal Institute of Technology, Stockholm, Sweden; ${ }^{2}$ Department of Rheumatology, Karolinska Institutet, Karolinska University Hospital, Stockholm, Sweden; ${ }^{3}$ Department of Orthopaedics, Karolinska University Hospital, Stockholm, Sweden

Arthritis Res Ther 2005, 7(Suppl 1):P116 (DOI 10.1186/ar1637)

Background The synovial membrane is not entirely homogeneous, leading to a local variability within a joint. Synovial tissue can be retrieved from patients in a number of ways (e.g. open surgery, blind needle biopsy, and arthroscopy).

Aim To study inter-patient versus intra-patient variability in gene expression in rheumatoid arthritis (RA) synovial tissue.

Patients and methods Orthopaedic samples Three synovial biopsies were taken from three RA patients (patients 1-3) at joint replacement surgery. All nine biopsies were divided into three, creating nine samples from each joint, and a total of 27 samples.

Arthroscopic samples Two to four synovial biopsies were sampled at arthroscopy from four RA patients (patients 4-7) with an inflamed knee joint. Multiple samples were taken from different sites in all patients.

Handling of synovial tissue and microarray analysis All samples were snap frozen and stored at $-80^{\circ} \mathrm{C}$. Following RNA extraction (at $4^{\circ} \mathrm{C}$ ), reverse transcription, cDNA amplification and labelling, each sample was hybridised in duplicate against a reference, on a cDNA array locally produced, representing 18,139 unique genes. After data filtering, genes were defined as differentially expressed (DE) if they had a B score $>0$ and a fold change $>2$. Several hierarchical clusterings were performed to obtain an overview of the data. DE genes in the samples were used to compare the variation between samples from one biopsy, the varia- tion between biopsies from one joint and the variation between biopsies from different patients. Clustering also allowed comparisons between the sampling techniques.

Results In the orthopaedic subset, variation between samples within one biopsy (patients 1-3) was between $1.2 \%$ and $6.7 \%$ of analysed genes. The amount of DE genes between biopsies was between $1.2 \%$ and 5.9\%. Hierarchical clustering of biopsies showed more similarities within a patient than between patients, except for one biopsy. One biopsy from patient 1 clustered with biopsies from patient 2 because the other two biopsies from patient 1 had higher fat cell content (confirmed microscopically) than any of the other biopsies. This was confirmed by gene expression profiles with activity in the fatty acid and lipid metabolism. When these genes were removed from the analysis, the samples clustered perfectly according to patient. The analysis of the arthroscopic subset (patients 4-7) showed a variation of DE genes between biopsies of $0.3-1.6 \%$, and a perfect matching in cluster analysis with all biopsies from the same patient clustering together, without any further labouring with data.

Table 1

\begin{tabular}{lccc}
\hline & $\begin{array}{c}\text { Average sample } \\
\text { weight }(\mathrm{mg})\end{array}$ & $\begin{array}{c}\text { Total RNA } \\
\text { yield (mg) }\end{array}$ & $\begin{array}{c}\text { RNA yield per } \\
\text { milligram of tissue (mg) }\end{array}$ \\
\hline Patients 1-3 & 32.9 & 14,808 & 502 \\
Patients 4-7 & 19.0 & 9834 & 407 \\
\hline
\end{tabular}

Conclusions This study shows that inter-patient variability is greater than intrapatient variability in gene expression, in RA synovial tissue. We found that one biopsy is enough to represent one patient as they clustered nicely together, with one exception, which was related to high content of fat cells in the biopsy. Variability is in the same order of magnitude in samples close to each other as compared with those further apart within the same patient.

\section{P117}

Association of FCGR2A and Fc gamma receptor haplotypes with Spanish polymyalgia rheumatica and giant cell arteritis AW Morgan', MA Gonzalez-Gay², JI Robinson', SJ Babbage1, L Haroon-Rashid' ${ }^{1}$, AH Hajeer ${ }^{3}$, WER Ollier ${ }^{3}$, JD Isaacs ${ }^{\mathbf{1}, \mathbf{4}}$

${ }^{1}$ Academic Unit of Musculoskeletal Disease and Molecular Medicine Unit, University of Leeds, UK; ${ }^{2}$ Rheumatology Division, Hospital Xeral-Calde, Lugo, Spain; ${ }^{3}$ The Centre for Integrated Genomic Medical Research, The University of Manchester, UK; ${ }^{4}$ School of Clinical Medical Sciences (Rheumatology),

University of Newcastle-Upon-Tyne, UK

Arthritis Res Ther 2005, 7(Suppl 1):P117 (DOI 10.1186/ar1638)

Background Polymyalgia rheumatica (PMR) and giant cell arteritis (GCA) are two inflammatory diseases that are thought to arise from a complex interaction between multiple genetic factors and environmental triggers. We have previously demonstrated an association between the $F_{c}$ gamma receptor ( $\left.F_{c} \gamma R\right)$ genetic locus on chromosome 1q22-23 and rheumatoid arthritis, and have now extended our study to include other inflammatory/autoimmune diseases.

Methods The FCGR2A-131H/R, FCGR3A-158F/V and FCGR3B-NA1/NA2 functional polymorphisms and a novel FCGR2B $3^{\prime}$-UTR polymorphism were examined for association with PMR $(n=69)$ and GCA $(n=83)$ in two well-characterised clinical cohorts from Northern Spain. In view of the close molecular proximity between FCGR2A, FCGR3A, FCGR3B and FCGR2B we have examined $F_{c} \gamma R$ haplotypes for association with rheumatoid arthritis. Pairwise disequilibrium coefficients $\left(D^{\prime}\right)$ were initially calculated in 115 matched controls. The EHPlus program was used to estimate haplotype frequencies for patients and controls and to determine whether significant linkage disequilibrium was present. A likelihood ratio test is performed to test for differences between the haplotype frequencies in cases and controls. A permutation procedure implemented in this program enabled 1000 permutations to be performed on all haplotype associations to assess significance.

Results There was borderline linkage disequilibrium between FCGR2A and FCGR3A ( $\left.D^{\prime}=-0.313, P=0.03\right)$ with more significant disequilibrium between FCGR3A and FCGR3B $\left(D^{\prime}=-0.646, P=0.0001\right)$. There was a significant difference in the FCGR2A allele $(P=0.03)$ and genotype frequencies $(P=0.03)$ with GCA compared with controls. Specifically, there was an increase in the FCGR2A-131RR genotype in both the PMR (odds ratio $[O R]=2.15 ; 95 \%$ confidence interval $[\mathrm{Cl}]=1.0-4.7, P=0.05)$ and $\mathrm{GCA}(\mathrm{OR}=2.53,95 \%$ $\mathrm{Cl}=1.3-5.1, P=0.01)$ populations compared with controls.

Increased homozygosity for the FCGR2A-FCGR3A 131R-158F haplotype was found in $8 \%$ controls compared with $25 \%$ of PMR (OR $=4.20,95 \% \mathrm{Cl}=$ $1.3-13.1, P=0.01)$ and $24 \% \mathrm{GCA}(\mathrm{OR}=4.46,95 \% \mathrm{Cl}=1.5-3.3, P=0.004)$ individuals. Logistic regression analyses suggested that both FCGR2A and FCGR3A contributed to GCA susceptibility. In addition, homozygosity for the 
FCGR3A-FCGR3B 158F-NA2 haplotype was found in 25\% controls compared with $44 \%$ GCA subjects (OR $=2.35,95 \% \mathrm{Cl}=1.0-5.7, P=0.06)$. Logistic regression analyses suggested that FCGR3A was the most important site contributing to GCA susceptibility.

A subgroup of GCA patients who had experienced visual symptoms was examined as a marker of disease severity. The most significant findings were increased homozygosity of a FCGR2A-FCGR3A 131R-158F haplotype (8\% controls, 16\% GCA without visual manifestations and $50 \%$ GCA with visual manifestations $[\mathrm{OR}=12.86,95 \% \mathrm{Cl}=1.3-128.2, P=0.03])$. Additionally, the FCGR3AFCGR3B haplotype was found in $11 / 13$ individuals who sustained visual manifestations. Homozygosity was seen in $25 \%$ controls, $27 \%$ GCA without eye involvement and 63\% GCA with visual manifestations $(\mathrm{OR}=5.83,95 \% \mathrm{Cl}=$ $0.9-38.9, P=0.08$ ). For each haplotype logistic regression analyses suggested FCGR3A was the most important site.

Conclusions We have demonstrated that FCGR2A may contribute to the 'susceptibility' of PMR and GCA in this Spanish population. The increased association observed with a FCGR2A-FCGR3A haplotype suggests the presence of additional genetic polymorphisms in linkage disequilibrium with this haplotype that may contribute to disease susceptibility. In addition, FcyR haplotypes may potentially define a subpopulation of individuals at greater risk of vascular occlusion. These findings may ultimately provide new insights into disease pathogenesis. Acknowledgement This work was funded by The Health Foundation.

\section{P118}

Noggin haploinsufficiency influences severity of arthritis in different mouse models

\section{Daans, RJU Lories, I Derese, P Tylzanowski, FP Luyten}

Laboratory for Skeletal Development and Joint Disorders, Department of

Rheumatology, University Hospitals Leuven, Katholieke Universiteit Leuven, Belgium Arthritis Res Ther 2005, 7(Suppl 1):P118 (DOI 10.1186/ar1639)

Introduction The severity and outcome of chronic arthritis is determined by the balance between destructive and homeostatic or anabolic molecular signaling pathways. Increasing evidence suggests a role for embryonic signaling pathways, essential for development and growth, in the maintenance of tissue homeostasis and in the induction of repair. Untimely or inappropriate activation of such pathways may also have a role in the progression of some diseases. Bone morphogenetic proteins (BMPs), originally identified as protein factors that induce a cascade of endochondral bone formation, have critical functions in cell proliferation, differentiation, adhesion and death. We have previously identified different BMPs in patients with chronic arthritis [1]. In this study, we evaluate the effect of shifting the balance in BMP signaling by reducing endogenous levels of noggin, a BMP antagonist, in a mouse model of systemic auto-immune arthritis (collageninduced arthritis $[\mathrm{ClA}]$ ), a model of cartilage destruction (methylated BSA [mBSA]-induced mono-arthritis) and a model of ankylosing enthesitis and spondyloarthritis (spontaneous arthritis in male DBA/1 mice).

Materials and methods Heterozygous mice with a targeted inactivation of the noggin gene and insertion of a $\beta$-galactosidase reporter (noggin+/LacZ) (a gift from $\mathrm{R}$ Harland, Berkeley, USA), originally in the C57/BI6 background, were backcrossed into the DBA/1 background for more than eight generations. mBSAinduced arthritis was induced by intra-articular mBSA injection on day 1 , followed by injection of subcutaneous IL-1 on three consecutive days. Mice were sacrificed at day 7. Severity of disease was determined with a histological score based on exsudate, inflammatory cell infiltration in the synovium, pannus formation, cartilage and bone destruction. Cartilage destruction and proteoglycan loss was further quantified using safranin $\mathrm{O}$ staining and digital image analysis of the articular surfaces from tibia, patella and femur. CIA was induced by allo-immunisation with chicken type II collagen emulsified in complete Freund adjuvant on day 1, followed by intraperitoneal booster injection of type II collagen on day 21. Arthritis characterized by ankylosing enthesitis is spontaneously occurring in aging male DBA/1 mice after grouped caging ( $>4$ males per cage) from the age of 14 weeks onwards. Incidence and severity of arthritis was assessed clinically and by histology in the latter two models. Presence of noggin was determined by immunohistochemistry in wild-type mice and by LacZ staining in noggin+/LacZ mice.

Results Noggin haploinsufficiency did not affect the incidence or clinical severity of CIA in DBA/1 mice. No differences in histological severity were seen. The histological severity of mBSA-induced monoarthritis in noggin ${ }^{+/ L a c Z}$ mice was similar to that of wild-type mice. However, cartilage destruction as determined by digital image analysis of proteoglycan content was significantly reduced in noggin ${ }^{+/ L a c Z}$ mice suggesting a protective role for BMP signaling. Disease incidence and severity of spontaneous arthritis was similar in noggin ${ }^{+/ L a c Z}$ and wild-type DBA/1 mice. However, histological analysis of this arthritis showed a slower disease progression in the haploinsufficient mice. Progression of ankylosing enthesitis, in particular chondrocyte hypertrophy and new bone formation, was delayed as compared with wild-type mice. The involvement of noggin in these processes was confirmed by immunohistochemistry for noggin in wild-type mice and LacZ staining in noggin illacZ $_{\text {mice. }}$
Conclusions Noggin haploinsufficiency has a protective effect on proteoglycan loss in a mouse model of cartilage destruction. Noggin haploinsufficiency also slows the progression of ankylosing enthesitis in a mouse model of spondyloarthritis. The role of noggin in ankylosing enthesitis is similar to what is seen during endochondral bone formation in development. These data provide further evidence that embryonic molecular signaling pathways, and BMP signaling in particular, are involved in the balance between tissue destruction, homeostasis and repair.

Reference

1. RJ Lories, et al:: Bone morphogenetic proteins 2 and 6 , expressed in arthritic synovium, are regulated by proinflammatory cytokines and differentially modulate fibroblast-like synoviocyte apoptosis. Arthritis Rheum 2003, 48:2807-2818.

Acknowledgements $M D$ and RL contributed equally to this work. RJUL is the recipient of a post-doctoral fellowship from the Fund for Scientific Research Flanders. This work was supported by research grant 0.390 .03 from the Fund for Scientific Research Flanders and a Bristol-Myers-Squibb EULAR Young Investigator Award to RJUL.

\section{P119}

Toll-like receptor expression in synovial fibroblasts and normal skin fibroblasts and induction of matrix metalloproteinase expression by various Toll-like receptor ligands D Kyburz', F Brentano', O Schorr'1, BA Michel', RE Gay', S Gay'

Center of Experimental Rheumatology, University Hospital, Zurich, Switzerland Arthritis Res Ther 2005, 7(Suppl 1):P119 (DOI 10.1186/ar1640)

Toll-like receptors (TLRs) are pattern recognition receptors of the innate immune system. TLR2 has been demonstrated to be expressed in synovial tissue of patients with rheumatoid arthritis (RA) as well as in cultured synovial fibroblasts of patients with RA and osteoarthritis. However, it is not known whether other members of the TLR family are expressed in synovial fibroblasts. We have therefore examined the expression of TLR1-TLR9 in these cells and performed stimulation experiments using specific TLR ligands. TLR and matrix metalloproteinase (MMP) mRNA expression was analysed by real-time PCR using 18S-cDNA as an internal control. A difference of five or more cycles between cDNA of the samples and non-RT control was considered to be the detection limit. While all fibroblasts expressed TLR1-TLR6, the expression of TLR2 and TLR3 was significantly higher in synovial fibroblasts from patients with RA as compared with normal skin fibroblasts. TLR7, TLR8 and TLR9 mRNA did not reach the detection level. To assess the function of these TLRs, cultured synovial and skin fibroblasts were stimulated with the TLR ligands bacterial lipopeptide, poly (I:C), lipopolysaccharide and flagellin. All ligands stimulated the expression of MMP1, MMP3, MMP9 and MMP13 to a certain extent. Whereas the TLR2 ligand bacterial lipopeptide preferentially induced the expression of MMP1 and MMP3, the TLR3 ligand poly (I:C) was a more efficient inducer of MMP13 and MMP3 and also induced MMP1 to a lesser extent. Normal skin fibroblasts expressed significantly lower levels of MMP3 and MMP13 mRNA as compared with synovial fibroblasts of patients with RA. Our data extend previous reports on the expression of TLRs on synovial fibroblasts to include TLR1-TLR6 and document that these TLRs are functional. Depending on the TLR ligand used, MMP expression is differentially induced. This supports the notion that activation of TLR signalling pathways might contribute to joint inflammation and destruction in RA.

\section{P120}

\section{Exploring mechanisms of inflammatory hyperalgesia in murine collagen-induced arthritis}

JJ Inglis', P Anand'2, P Facer'2, G Criado', M Feldmann', RO Williams'

${ }^{1}$ Kennedy Institute of Rheumatology, Imperial College London, UK; ${ }^{2}$ Neuroscience and Psychological Medicine, Imperial College London, UK Arthritis Res Ther 2005, 7(Suppl 1):P120 (DOI 10.1186/ar1641)

Rheumatoid arthritis (RA) is an autoimmune inflammatory disorder, characterised by joint swelling and diffuse chronic pain. RA patients display both hyperalgesia (an exaggerated painful response to a noxious stimulus) and allodynia (a painful response to a non-noxious stimulus). Collagen-induced arthritis (CIA) in the mouse is a well-established model of RA that may be useful in the study of inflammatory hyperalgesia.

The aim of this study was to establish CIA as a model for studying inflammatory hyperalgesia, and to assess cellular changes that occur in the nociceptive system during the course of arthritis.

Arthritis was induced by injection of $2 \mathrm{mg} / \mathrm{ml}$ bovine type II collagen in complete Freund's adjuvant into the base of the tail of male DBA/1 mice $(n=30)$. Behavioural analysis was performed for 28 days following arthritis onset. Mechanical and thermal hyperalgesia was assessed using the Plantar Von-Frey microprocessor system and the Hargreaves Plantar test, respectively. Animals were sacrificed at intervals after arthritis onset, and the lumbar spinal cord was collected and immunostained for astrocytes using antibodies to glial fibrillary acidic protein. 
Figure 1

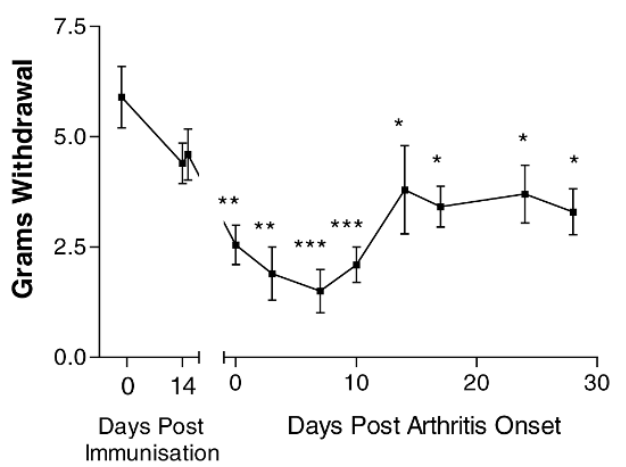

The development of mechanical hyperalgesia in mice with collagen-induced arthritis.

Prior to onset of inflammation (post immunisation), little hyperalgesia occurred. Following arthritis onset there was a rapid decrease in both mechanical (Fig. 1) and thermal thresholds, reaching a maximum at 7-10 days after onset. Thermal threshold returned to naîve levels 24 days following arthritis onset, while mechanical hyperalgesia remained throughout the study.

Increased glial fibrillary acidic protein expression was detected in the spinal cord from the day of arthritis onset, indicating that astrocytic activation occurs in CIA. This is in agreement with a well-established inflammatory model used for the study of pain, complete Freund's adjuvant-induced monoarthritis.

Our results indicate that CIA can be used in the study of pain, and that changes occur in the nociceptive system during inflammation. As CIA is a well-established and validated model of RA, it may be of benefit to test analgesics aimed at inflammatory pain in this model and to understand the mechanisms involved in inflammatory hyperalgesia.

Acknowledgement This work is supported by GlaxoSmithKline.

\section{P121}

\section{Association of FCGR3A and FCGR3B haplotypes with} rheumatoid arthritis and primary Sjögren's syndrome S Mackie', Jl Robinson 1,2, JH Barrett ${ }^{3}$, CA Lawson'1,2, S Martin 1,2, L Haroon-Rashid1,2, D Cooper1,2, SJ Bowman 4, CT Pease1, PG Conaghan', M Green', M Quinn', JD Isaacs 1,5, P Emery', AW Morgan 1,2

${ }^{1}$ Academic Unit of Musculoskeletal Disease, University of Leeds, UK; ${ }^{2}$ Molecular Medicine Unit, University of Leeds, UK; ${ }^{3}$ Cancer Research UK, Genetic Epidemiology Division, University of Leeds, UK: ${ }^{4}$ Rheumatology Department, University Hospital Birmingham (Selly Oak), Birmingham, UK; ${ }^{5}$ School of Clinical Medical Sciences (Rheumatology), University of Newcastle-Upon-Tyne, UK Arthritis Res Ther 2005, 7(Suppl 1):P121 (DOI 10.1186/ar1642)

Background Rheumatoid arthritis (RA) is an autoimmune disease that is thought to arise from a complex interaction between multiple genetic factors and environmental triggers. We have previously demonstrated an association between a Fc gamma receptor (FcyR) haplotype and RA in a cross-sectional cohort of RA patients. We have sought to confirm this association in an inception cohort of RA patients and matched controls. We also extended our study to investigate a second autoantibody associated rheumatic disease, primary Sjögren's syndrome (PSS).

Methods The FCGR3A-158F/V and FCGR3B-NA1/NA2 functional polymorphisms were examined for association in an inception cohort of RA patients $(n=448)$, and a well-characterised PSS cohort $(n=83)$ from the United Kingdom. Pairwise disequilibrium coefficients $\left(D^{\prime}\right)$ were calculated in 267 Blood Service healthy controls. The EHPlus program was used to estimate haplotype frequencies for patients and controls and to determine whether significant linkage disequilibrium was present. A likelihood ratio test is performed to test for differences between the haplotype frequencies in cases and controls. A permutation procedure implemented in this program enabled 1000 permutations to be performed on all haplotype associations to assess significance.

Results There was significant linkage disequilibrium between FCGR3A and FCGR3B $\left(D^{\prime}=-0.445, P=0.001\right)$. There was no significant difference in the FCGR3A or FCGR3B allele or genotype frequencies in the RA or PSS patients compared with controls. However, there was a significant difference in the FCGR3A-FCGR3B haplotype distributions with increased homozygosity for the FCGR3A-FCGR3B 158V-NA2 haplotype in both our inception RA cohort (odds ratio $=2.15,95 \%$ confidence interval $=1.1-4.2 P=0.027$ ) and PSS (odds ratio $=2.83,95 \%$ confidence interval $=1.0-8.2, P=0.047)$ compared with controls.
The reference group for these analyses comprised individuals who did not possess a copy of the FCGR3A-FCGR3B $158 \mathrm{~V}$-NA2 haplotype.

Conclusions We have confirmed our original findings of association between the FCGR3A-FCGR3B 158V-NA2 haplotype and RA in a new inception cohort of RA patients. This suggests that there may be an RA-susceptibility gene at this locus. The significant increased frequency of an identical haplotype in PSS suggests the FcyR genetic locus may contribute to the pathogenesis of diverse autoantibody-mediated rheumatic diseases.

Acknowledgements SM and JIR are joint first authors. This work was funded by the Arthritis Research Campaign.

\section{P122}

Non-traditional risk and protective factors for cardiovascular disease in systemic lupus erythematosus A Cederholm ${ }^{1}$, E Svenungsson ${ }^{2}$, D Stengel ${ }^{3}$, G-Z Fei', AG Pockley ${ }^{4}$, E Ninio ${ }^{3}$, J Frostegård1

${ }^{1}$ Department of Medicine, CIM and CME, Karolinska University Hospital, Huddinge, Sweden; ${ }^{2}$ Department of Rheumatology, Karolinska University Hospital, Solna, Sweden; 3INSERM U 525/IFR14 Cœur Muscle Vaisseaux and Université PM Curie/Faculté de Médecine Pitié-Salpétrière, Paris, France; ${ }^{4}$ Division of Clinical Sciences (North), University of Sheffield, UK Arthritis Res Ther 2005, 7(Suppl 1):P122 (DOI 10.1186/ar1643)

Background There is an important inflammatory and autoimmune component to atherosclerosis and cardiovascular disease (CVD). It is therefore interesting that the risk of CVD is so exceedingly high in patients with systemic lupus erythematosus (SLE).

Objective To investigate the role of non-traditional risk and protective factors for CVD related to inflammation and immune activation in SLE-associated CVD.

Methods Twenty-six women ( $52 \pm 8.2$ years) with SLE and a history of CVD (SLE cases) were compared with 26 age-matched women with SLE and no clinical manifestations of CVD (SLE controls) and 26 age-matched healthy women (population controls). As a surrogate measure of atherosclerosis, common carotid intima-media thickness and plaque occurrence were detected by B-mode ultrasound. Circulating levels of oxidized low-density lipoprotein (LDL) were measured by a mAb-4E6-based competition ELISA. Several novel non-traditional risk factors identified by us and others were determined in serum: heat shock protein (HSP)-related factors (Hsp60, Hsp70 and anti-human Hsp60, anti-human Hsp70 and anti-mycobacterial Hsp65 antibody levels), platelet activating factor-acetylhydrolase activity (PAF-AH) and secretory phospholipase A2 GIIA (sPLA2). Antibodies against endothelial cells $(\mathrm{aEC})$ were measured by in-house ELISA.

Results The circulating levels of oxidized LDL and activity levels of PAF-AH, but not HSP-related measurements, aEC antibodies or SPLA2, were significantly raised in SLE cases. Oxidized LDL levels and PAF-AH discriminated between SLE controls and SLE cases $(P=0.0282$ or $P=0.008$, respectively). PAF-AH was significantly associated with LDL and cholesterol among SLE/CVD $(r=0.50$, $P=0.009$ and $r=0.54, P=0.004$ ), but not in the other groups tested.

Conclusion The difference in oxidized LDL levels between SLE cases and SLE controls may indicate that increased LDL modification by oxidation is one the underlying factors implicated in SLE-related CVD. The association between PAF$\mathrm{AH}$ and $\mathrm{LDL}$ adds support to the notion that PAF-AH, which binds to LDL, might contribute to atherogenesis in SLE patients. We cannot exclude that PAF-AH is simply a secondary marker of other as yet unknown processes The role of HSPrelated measurements in CVD in general is complex, since aHSP65 appears to be atherogenic while HSP70 may be protective. In the present cross-sectional study, the HSP-related measurements and those of aEC and sPLA2 were not associated with SLE.

\section{P123}

Immunomodulatory effect of unpulsed-immature dendritic cells in collagen-induced arthritis

PL Plence ${ }^{1}$, F Apparailly', LM Van Duivenvoorde ${ }^{2}$, LM Charbonnier ${ }^{1}$, REM Toes ${ }^{2}$, C Jorgensen 1

${ }^{1}$ INSERM U475, Hopital Lapeyronie, service Immuno Rhumatologie, Montpellier France; ${ }^{2}$ Department of Rheumatology, Leiden University Medical Center, Leiden, The Netherlands

Arthritis Res Ther 2005, 7(Suppl 1):P123 (DOl 10.1186/ar1644)

Objective Dendritic cells (DC) play an important role for initiation and regulation of immune response. Recent experimental evidence points out the fact that immature dendritic cells (iDC) can mediate tolerance, presumably by the induction of regulatory $T$ cells. Therefore, we explored the effects of iDC injection on the development of collagen-induced arthritis in mice and compared them with tumour necrosis factor-matured DC, which we were previously shown efficient in collagen-induced arthritis.

Methods Murine bone marrow-derived DC where cultured in the presence of GM-CSF and IL-4 for 6 days and were incubated or not for 24 hours with bovine type II collagen (bCII). The immature phenotype of DC was controlled by flow 
cytometry, IL-12p40 secretion and MLR assays. The iDC were repetitively injected 7,5 and 3 days before arthritis induction with bCll. Mice were boosted on day 21 and the disease course was monitored until day 50. Paw swellings were measured over time, and radiological and histological analyses of paws were performed at euthanasia. The antigen-specific T-cell proliferative response of spleen cells, the Th1/Th2 cytokine production, and the serum levels of $\operatorname{lgG}_{1} / \operatorname{lgG}_{2 \mathrm{a}}$ anti-bCll antibodies were measured.

Results The repetitive injection of tumour necrosis factor alpha-modulated DC loaded with bCll protects the mice from severe arthritis. This protective effect is antigen dependent of the DC loading with bCll. The immunosuppressive effect is associated with a decrease of the anti-bCll antibodies from the $\operatorname{lgG}_{2 a}$ isotype, together with the inhibition of interferon gamma synthesis and the decrease proliferation of bCll-specific T cells.

Our data show that repetitive injection of immature unloaded DC protects the animals significantly as $70 \%$ of the mice did not develop clinical signs of the disease. We quantitated the various regulatory populations in the vaccinated mice, and observed in the liver and spleen an increase of the T-cell population expressing the CD49b molecule (DX5 cells). The relevance of this population in induced protection is under investigation.

Conclusion The use of iDC to modulate the autoimmune response is a rational approach for cell therapy in diseases such as rheumatoid arthritis.

\section{P124}

Cytokine expression was assessed during antigen-induced arthritis in the synovial membrane, inguinal lymph node, and spleen using competitive RT-PCR and sandwich ELISA RW Kinne, D Pohlers, A Siegling, E Buchner, CB Schmidt-Weber, E Palombo-Kinne, F Emmrich, R Bräuer

Experimental Rheumatology Unit, Friedrich Schiller University, Jena, Germany Arthritis Res Ther 2005, 7(Suppl 1):P124 (DOI 10.1186/ar1645)

In the synovial membrane, early mRNA elevations of IL-1 $\beta$ and IL-6 (6 hours; 450fold and 200-fold, respectively) positively correlated with the joint swelling. A sixfold tumour necrosis factor alpha increase was not significant. Not only IL-2 and interferon gamma (IFN- $\gamma)$ (10 $0^{4}$-fold and 200-fold, respectively), but also IL-5 and IL-10 increased acutely ( 6 hours-day 1 ; threefold and 35 -fold, respectively). In general, the protein levels for IL-1 $\beta$, IL-6, tumour necrosis factor alpha, IFN- $\gamma$, IL-4, and IL-10 (increase between fourfold and 15-fold) matched the course of mRNA expression. In the inguinal lymph node there were early mRNA elevations of IL-6 (6 hours; 2.5-fold; positively correlated with the joint swelling) and IL-2 (6 hours; fourfold), as well as later rises of IL-4 and IL-5 (day 3; 2.5 -fold and fourfold, respectively). At the protein level no significant elevations were observed in comparison with day 0 , except for IL-1 $\beta$ (day 6) and IL-10 (day 1).

In the spleen, there were significant mRNA elevations at 6 hours of IL-1 $1 \beta$ (1.5fold), IL-6 (fourfold; positively correlated with the joint swelling), IFN- $\gamma$ (threefold), and IL-2 (sevenfold to 10-fold). IL-5 and IL-10 (twofold and threefold, respectively) peaked from 6 hours to day 3. Increases at the protein level were significant compared with day 0 only in the case of IL-2 (day 6).

By day 6 (transition to the chronic phase) the mRNA for cytokines declined to or below pre-arthritis levels in all organs, except for IL-1 $1 \beta$ in the synovial membrane and IL-6 in the spleen. antigen-induced arthritis is thus characterized by: early synovial activation of macrophages, Th1-like, and Th2-like cells; late, well-segregated Th2-like responses in the inguinal lymph node; late, overlapping Th1like/Th2-like peaks in the spleen; and chronic elevation of synovial IL-1 $\beta$ mRNA and spleen IL-6 mRNA.

\section{P125}

Long-term immune reconstitution after autologous stemcell transplantation for severe autoimmune diseases T Alexander ${ }^{1}$, G Massenkeil'2, E Gromnica-Ihle ${ }^{3}$, GR Burmester ${ }^{1}$, A Radbruch 4 , R Arnold ${ }^{2}$, F Hiepe ${ }^{1}$, A Thiel ${ }^{4}$

${ }^{1}$ Department of Rheumatology and Clinical Immunology, University Hospital Charité, Berlin, Germany; 2Department of Hematology and Oncology, University Hospital Charité, Berlin, Germany; ${ }^{3}$ Rheumaklinik Berlin Buch, Germany 4 German Rheumatism Research Center Berlin, Germany

Arthritis Res Ther 2005, 7(Suppl 1):P1 25 (DOI 10.1186/ar1646)

Objectives We performed a detailed analysis of the newly developing immune system in patients treated with autologous stem-cell transplantation (ASCT) for severe autoimmune diseases with special respect to the origin and the kinetics of repopulating B-cell and T-cell subsets in correlation to the clinical response.

Methods Peripheral blood lymphocytes were analyzed using multiparameter flow cytometry, including monitoring of the TCR-Vbeta repertoire on CD4 ${ }^{+}$Th cells. Thymic activity was determined assessing absolute counts of peripheral blood CD31+ thymic naive Th cells.

Patients and results Thirteen patients with a median follow-up of 54 months have so far been included in the trial: polychonditis $(n=1)$, systemic lupus ery- and multiple sclerosis $(n=2)$. Clinical remission has been achieved in all patients with polychondritis $(n=1)$, SLE $(n=6)$ and multiple sclerosis $(n=2)$ whereas we observed progression of disease in all patients with SSc $(n=3)$ and panniculits $(n=1)$. One SLE patient relapsed after being free of any clinical and serological symptoms for 17 months.

T-lymphocyte compartments reconstituted functionally in all patients, indicated by the reappearance and persistence of $C D 45 R A^{+} C D 31^{+}$thymic naive Th cells in high numbers with high levels of T-cell receptor excision circles and restored diversity of the Th-cell-receptor repertoire. In terms of Th-cell memory no autoreactive Th cells could be detected in responding patients. Reconstituted $\mathrm{B}$ cells were primarily of naive phenotype $\left(\operatorname{lgD}{ }^{+} C^{2} 2^{-}\right)$in the first $12-18$ months after ASCT. As a cellular marker for disease activity, frequencies of $\mathrm{CD} 19^{+} \mathrm{CD} 27^{++} \mathrm{CD} 20^{-}$plasma blasts, elevated in SLE patients prior to ASCT, normalized after treatment in all patients. In case of the non-responding patients with SSc, autoantibody titers were not affected by treatment. However, no major differences in the pattern of lymphocyte reconstitution could be detected as compared with responding patients. Conclusion The newly developing immune system after immunoablative therapy could be characterized as 'juvenile' in all patients. However, in some patients the regeneration of a naive immune system alone seems not to be sufficient to regain self-tolerance. We conclude that both the resetting of central and peripheral tolerance and the efficient eradication of autoreactive lymphocytes seem to be a prerequisite for stable induction of remission.

\section{P126}

The effect of B-cell depletion with an anti-CD20 antibody on the immunoglobulin heavy-chain repertoire in a patient with rheumatoid arthritis

\section{A-S Rouzière', C Kneitz' ${ }^{1}$, T Dörner' ${ }^{2}$, H-P Tony ${ }^{1}$}

${ }^{1}$ Department of Medicine II, Rheumatology and Clinical Immunology, University of Wuerzburg, Germany; ${ }^{2}$ Charité University Hospital, Berlin, Germany

Arthritis Res Ther 2005, 7(Suppl 1):P126 (DOI 10.1186/ar1647)

Purpose B-cell depletive therapy has been described to have beneficial effects to patients suffering from rheumatoid arthritis (RA). Nevertheless, the role of $B$ cells in pathogenesis is not clear. In particular, it is not known whether B-cell depletion causes changes in the B-cell repertoire after peripheral regeneration. Therefore we analysed the distribution of the immunoglobulin heavy-chain (Ig-VH) genes usage of a RA patient before, 7 months and 17 months after anti-CD20 therapy with rituximab.

Methods A patient with active RA has been treated with four weekly doses of $375 \mathrm{mg} / \mathrm{m}^{2}$ rituximab. During the treatment phase, prednisolone was elevated up to $12.5 \mathrm{mg}$ daily for 4 weeks. After that, the patient remained on a stable dose of $5 \mathrm{mg}$ prednisolone per day without further anti-proliferate treatment. Ig-VH genes were amplified from genomic DNA of peripheral B cells by nested PCR using family-specific oligonucleotides. PCR products were subcloned and the sequences of more than 500 clones were analysed.

Results Following antibody treatment, the patient experienced a good clinical response lasting over 1 year. Before therapy, the overall $\mathrm{VH}$ gene usage of the RA patient was largely similar to the distributions expected from published data for normal individuals, except for certain genes (4-34, 1-69, 3-07), which have already been reported with a biased distribution in autoimmune disorders. In addition, several clonally related sequences were found in the $\mathrm{VH} 5$ family.

B-cell regeneration occurred 7 months after anti-CD20 antibody therapy. Significant changes in the distribution of different genes could be observed: 4-34 and 1-69 decreased to a proportion that would be expected in healthy individuals. Clonally related sequences were not found anymore. A greater overall variety of the $\mathrm{VH}$ gene segments used was observed, in particular in the larger families like VH3 and VH4. Interestingly, somatic hypermutation was significantly enhanced at the time point of B-cell regeneration.

Seventeen months after therapy, the general distribution of the $\mathrm{VH}$ genes was comparable with that observed before treatment, except for some genes (4-34 remained at the level found after therapy). No clonally related sequences were found. Somatic hypermutation was significantly reduced to the frequency determined before therapy.

Conclusion B-cell depletion by anti-CD20 monoclonal antibody leads to a significant modulation of the expressed immunoglobulin- $\mathrm{VH}$ gene repertoire after $\mathrm{B}$-cell regeneration, which is accompanied with a clinical benefit in a single patient.

P127

\section{Direct analysis of bone turnover in experimental arthritis} K Polzer, B Tuerk, K Redlich, S Hayer, J Smolen, G Schett

Division of Rheumatology, Department of Internal Medicine III, Medical University of Vienna, Austria

Arthritis Res Ther 2005, 7(Suppl 1):P127 (DOI 10.1186/ar1648)

Introduction Chronic arthritis usually leads to loss of periarticular bone. Inflammatory bone loss results from an imbalance between bone formation and bone resorption. Recent research has focused on the role of osteoclast formation and 
bone resorption in arthritis. However, bone resorption cannot be seen isolated since it is closely linked to bone formation and changes in bone formation may also affect inflammatory bone loss.

Methods To assess bone resorption and bone formation simultaneously, we developed a histological technique that allowed visualization of osteoblast function by in situ hybridization for osteocalcin and osteoclast function by histochemistry for tartrate-resistant acid phosphatase simultaneously on the same section. Paw sections from human tumor necrosis factor transgenic mice, which develop erosive arthritis, were analyzed at three different sites: subchondral bone erosions, adjacent Haversian bone channels and endosteal regions distant from bone erosions.

Results In subchondral bone erosions, resorption sites were far more common than formation sites. Thus, both the areas covered by osteoclasts as well as numbers of osteoclasts showed a fivefold increase compared with the area covered by osteoblasts and osteoblast numbers, respectively. In contrast, adjacent Haversian channels showed a completely different bone turnover, with functional osteoblasts significantly more frequent than osteoclasts. Bone turnover in Haversian channels was even higher $(50 \%$ bone surface covered by osteoclasts or osteoblasts) than in subchondral bone erosions (30\%). At endosteal sites distant from bone erosions, bone turnover was far lower $(6 \%)$ and again bone formation exceeded bone resorption.

Conclusion These data indicate that bone resorption dominates at skeletal sites close to synovial inflammatory tissue, whereas more distant sites such as the Haversian channels attempt to counterregulate this process by increased bone formation.

\section{P128}

\section{Antibodies to ferritin in rheumatoid arthritis are associated} with disease severity D Mewar', DJ Moore', PF Watson'3 , AG Wilson'1

${ }^{1}$ Division of Genomic Medicine, University of Sheffield, UK; ${ }^{2}$ Department of Radiology, Royal Hallamshire Hospital, Sheffield, UK; ${ }^{3}$ Division of Clinical Sciences North, University of Sheffield, UK Arthritis Res Ther 2005, 7(Suppl 1):P128 (DOI 10.1186/ar1649)

Background and objectives $A$ number of autoantibodies have been described in individuals with rheumatoid arthritis (RA) leading to interest in their use as diagnostic or prognostic markers in RA as well as their pathogenic relevance. By immunoscreening of a phage-display expression cloning system with RA patient sera we isolated a cDNA clone encoding the ferritin $\mathrm{H}$ chain polypeptide. The objectives of this study were to establish the frequency and clinical associations of anti-ferritin antibodies in RA.

Methods We employed an ELISA for the measurement of anti-ferritin antibodies in RA sera. Briefly, 96-well plates were coated overnight with purified ferritin from equine spleen. Sera diluted one in 100 were reacted with the plates for 16 hours at $4^{\circ} \mathrm{C}$. After three washes, a goat anti-human lgG/horseradish peroxidase conjugate was used to detect bound IgG. The signal for each serum sample was normalised using a reference serum included on each plate. Anti-ferritin antibodies were measured in 291 subjects with RA, 73 healthy blood donors and 91 subjects with osteoarthritis. Antibody-positive and antibody-negative individuals were compared with respect to severity of disease as measured by modified Larsen's score, demographic variables, rheumatoid factor status and carriage of HLA DRB1 shared epitope alleles. Correlations were examined between antibody levels and severity of joint damage assessed by the Modified Larsen's score.

Results Using a cut-off index of three standard deviations above the mean of the control group, 49/291 (16.8\%) RA patients were positive versus 2/73 (2.7\%) healthy donors and $2 / 91$ subjects with osteoarthritis $(P<0.01)$. In six positive and six negative sera these findings were confirmed by western blotting. Anti-ferritin antibodies were more common in males with RA $(25.3 \%$ males versus $13.7 \%$ females, $P<0.02)$ and levels were positively associated with severity of joint damage $\left(r_{\mathrm{s}}=0.33, P=0.02\right)$.

Conclusions Anti-ferritin antibodies are present in a subset of individuals with RA and are associated with more severe joint damage. Ferritin is an abundant protein in serum and synovial fluid, and therefore ferritin/anti-ferritin immune complexes could form in these individuals and contribute to joint damage. An alternative possibility is that anti-ferritin antibodies could modulate the iron-binding properties of ferritin and so lead to the release of toxic-free iron in the joint. We are currently examining the frequency of anti-ferritin reactivity in patients with early RA at two timepoints in order to establish whether or not these antibodies are present in early disease or are simply a result of longstanding disease, as well as their frequency in other autoimmune diseases. These data will be presented.

\section{P129}

\section{Polymorphism within the telomeric MHC and expression of rheumatoid arthritis candidate genes D Mewar, I Marinou, JM Timms, R Kilding, AG Wilson}

Division of Genomic Medicine, University of Sheffield, UK

Arthritis Res Ther 2005, 7(Suppl 1):P129 (DOI 10.1186/ar1650)

Background and objectives Several recent studies have indicated a second susceptibility locus for rheumatoid arthritis (RA) in the telomeric MHC close to the tumour necrosis factor locus, a region containing a number of genes of unknown function including HLA-B associated transcripts 1 and 2 (BAT1 and $B A T 2)$ and lymphotoxin alpha (LTA). Several single-nucleotide polymorphisms have been described in this region; however, the influence of each on susceptibility is not known. In this study we sought to examine the association of individual single-nucleotide polymorphisms with expression of each mRNA transcript in response to an inflammatory stimulus.

Methods Peripheral blood mononuclear cells were collected from healthy blood donors and adherent cells were allowed to differentiate into macrophages by culture in the presence of $5 \%$ serum for 7 days. Cells were then stimulated with $100 \mathrm{ng} / \mathrm{ml}$ lipopolysaccharide and harvested at 4-hour and 18-hour timepoints. Total RNA was prepared using standard methods and reverse transcription performed with Impromll ${ }^{\mathrm{TM}}$ (Promega, Madison, WI, USA). The resulting CDNA was used as a template for real-time PCR using gene-specific primers for each gene. Each PCR product was detected with the double-stranded DNA-binding dye SYBR Green ${ }^{\circledR}$ I (Applied Biosystems, Foster City, CA, USA) and the ABI $7900 \mathrm{HT}$ sequence detection system (Applied Biosystems). Quantitation was performed using standard curves generated from plasmid DNA containing each cloned PCR product and $18 \mathrm{~S}$ was used as a loading control. Levels of mRNA transcripts were expressed in relative units (RU). Genotyping was performed for the BAT1 -991 (1751673), BAT2 (1688185), LTA (1720807) and TNF -308 (1718089) polymorphisms using TaqMan allelic discrimination assays. Expression levels of each mRNA transcript were compared between individuals of genotype 1.1, 1.2 and 2.2 using Kruskal-Wallis tests.

Results Expression of BAT1 mRNA transcripts was significantly less in BAT1-991 2.2 individuals $(n=6)$ compared with both $1.1(n=6)$ and $1.2(n=5)$ (median levels 0.22 RU compared with 0.76 RU and 1.1 RU, respectively) $(P=0.02)$. Expression of BAT2 transcripts was lower in 1.1 individuals $(n=9)$ compared with both $1.2(n=8)$ and $2.2(n=4)$ genotypes (median levels $0.43 \mathrm{RU}$ compared with $0.77 \mathrm{RU}$ and $0.82 \mathrm{RU}$, respectively) ( $P=$ not significant). For $L T A$ expression, individuals of genotype $2.2(n=4)$ produced the highest levels, those of genotype 1.1 $(n=6)$ the lowest, with those of 1.2 genotype $(n=6)$ intermediate (median levels 6.1 RU compared with 0.81 RU and 2.1 RU, respectively) $(P=0.02)$. Expression of all three transcripts had fallen to low levels by the 18-hour timepoint and no differences were apparent between individuals of different genotype.

Conclusions Polymorphism in the BAT1 -991, BAT2 and LTA genes are associated with levels of each mRNA transcript produced in response to an inflammatory stimulus. This suggests the possibility that they may be able to influence susceptibility to RA, and that the protein products they encode may play a role in inflammatory signalling. We are currently extending this genotype-phenotype analysis to the other members of this gene cluster particularly 1C7, LST1 and AIF1.

P130

\section{Synovial monocytes mediate Rap1-dependent oxidative stress in rheumatoid arthritis $\mathrm{T}$ lymphocytes} PH Remans' ${ }^{1}$ JM van Laar' ${ }^{2}$, ME Sanders', P-P Tak', KA Reedquist

${ }^{1} A M C$, Amsterdam, The Netherlands; 2 LUMC, Leiden, The Netherlands Arthritis Res Ther 2005, 7(Suppl 1):P130 (DOI 10.1186/ar1651)

Background Transient production of reactive oxygen species (ROS) plays an important role in optimizing transcriptional and proliferative responses to T-cell receptor signaling. Conversely, chronic oxidative stress leads to mitogenic hyporesponsiveness and enhanced transcription of inflammatory gene products. It has recently been demonstrated that constitutive activation of the smal GTPase Ras and simultaneous inhibition of Rap1 in synovial fluid (SF) T cells results in high intracellular ROS production, which is thought to underlie many of the functional abnormalities observed in these cells in rheumatoid arthritis.

Objectives To identify the factor(s) responsible for modulation of intracellular ROS production in synovial T lymphocytes.

Methods Purified rheumatoid arthritis peripheral blood (PB) T cells were incubated in the presence of different cytokines, in $50 \%$ autologous SF, or with autologous SF monocytes for 72 hours. Activation status of Ras and Rap1 GTPases were determined using activation-specific probes for these GTPases. Oxidation of the dye DCF by FACS analysis was used to measure intracellular ROS production.

Results Chronic stimulation of PB T cells for 72 hours with tumor necrosis factro alpha (TNF- $\alpha$ ) or $50 \%$ autologous SF resulted in a slight increase in basal ROS production, but did not increase intracellular ROS production to levels found in SF T cells. Exposure of PB T cells to SF (but not PB) monocytes for 72 hours, however, led to a strong increase in ROS production in PB T cells, comparable with ROS levels in SF T cells. Moreover, similar Rap1 inhibition as found in SF T cells were observed in PB T cells after exposure to SF monocytes. To demonstrate that the inhibition of Rap1 is critical in the subsequent increase in ROS production, PB T cells were nucleofected with the constitutive active isoform of Rap1 (RapV12). In RapV12 nucleofected PB T cells the SF monocyte-induced ROS production was prevented. Cell-cell contact is critical, since in PB T cells separated from SF monocytes by a transwell membrane, the inhibition of Rap1 was relieved, concomitant with an absence in excess ROS production. Additionally, we found that addition of $10 \mu \mathrm{g} / \mathrm{ml}$ recombinant CTLA-4-lg fusion protein 
also prevented oxidative stress in PB T cells exposed to SF monocytes, which suggested a central role for CD28. PB T cells were therefore stimulated with TNF- $\alpha$, interferon gamma, IL-1 $\beta$, or transforming growth factor beta, in the presence or absence of anti-CD28. Here we found that stimulation with anti CD28 by itself was sufficient to induce Rap1 inhibition and induce a moderate increase in ROS production. Co-incubation of PB T cells with TNF- $\alpha$ strongly enhanced the intracellular ROS production.

Conclusion In vitro exposure of PB T cells from rheumatoid arthritis patients to synovial monocytes leads to a strong increase in intracellular ROS production. This is mediated by simultaneous Ras activation and inhibition of Rap1. Where Ras can be activated by a variety of stimuli, Rap 1 inhibition is induced by SF monocytes through CD28 costimulatory signaling.

\section{P131}

\section{Molecular response to cartilage injury F Dell'Accio, C De Bari, C Pitzalis}

Department of Rheumatology, King's College London, UK

Arthritis Res Ther 2005, 7(Suppl 1):P131 (DOI 10.1186/ar1652)

Background Post-traumatic osteoarthritis (OA) represents $13 \%$ of all OA of the knee, $9 \%$ in the hip, and $73 \%$ of all OA of the ankle [1]. The molecular mechanisms that underlie the development of chronic joint surface degeneration following a localised, acute joint injury are not known. The aim of this study is to utilise the cartilage explant model system to identify molecular mechanism of cartilage damage and repair induced by acute mechanical injury.

Methods Explants of articular cartilage from preserved areas of the femoral condyles or the patellar groove of patients undergoing total knee prosthesis were cultured in vitro under different conditions. After 4 days in culture the samples were subjected or not to mechanical injury by performing full thickness cuts at a distance of $1 \mathrm{~mm}$. At different time-points the explants were partly snap-frozen for further histochemical/immunohistochemical evaluation and partly used for RNA extraction and RT-PCR analysis. The original cartilage was graded using the Mankin score.

Results Gene regulation was detected as early as 4 hours and lasting for at least 6 days after mechanical injury. BAX mRNA, possibly associated with apoptosis of chondrocytes, MMP-8, and TIMP-1 mRNA were upregulated in the injured explants. BMP-2 mRNA was also upregulated by mechanical injury. We could not detect changes upon damage of cell proliferation as measured by PCNA mRNA levels at the analysed time points.

Conclusions The explant model system represents a controlled experimental setup to study the molecular mechanisms of cartilage degeneration in post-traumatic $\mathrm{OA}$ and to identify potential molecular targets for its prevention.

Reference

1. Buckwalter JA, Saltzman C, Brown T, Schurman DJ: The impact of osteoarthritis: implications for research. Clin Orthop 2004, 427(Suppl):S6-S15.

\section{P132}

\section{Binding properties of anti-histone $\mathrm{H} 1$ antibodies suggest that nuclei of necrotic cells rather than cells undergoing apoptosis are engulfed in the LE cell phenomenon} E Feierl, JS Smolen, T Karonitsch, GH Stummvoll, H Ekhart, CW Steiner, M Aringer

Department of Rheumatology, Internal Medicine III, Medical University of Vienna, Austria

Arthritis Res Ther 2005, 7(Suppl 1):P132 (DOI 10.1186/ar1653)

Background The LE cell phenomenon is a classical test for systemic lupus erythematosus (SLE). Granulocytes engulf bodies, the nature of which has not been unequivocally demonstrated. Hypotheses range from isolated nuclei to intact cells undergoing apoptosis. Using the fact that it is autoantibodies to histone $\mathrm{H} 1$ that induce this phenomenon, which thus have to bind the material in question, we investigated histone $\mathrm{H} 1$ accessibility to antibodies.

Methods Polymorphonuclear cells (PMNC) were isolated by centrifugation between Percoll layers, and peripheral blood mononuclear cells over Ficoll Paque. Cells were either used while vital or after the induction of apoptosis (using gliotoxin for PMNC and actinomycin D for peripheral blood mononuclear cells and incubation overnight) or necrosis (heating to $70^{\circ} \mathrm{C}$ ). Apoptosis and necrosis were verified by both Annexin V/propidium iodide and TUNEL on a flow cytometer. Accessibility of histone $\mathrm{H} 1$ was likewise tested by fluocytometry, employing a monoclonal anti-histone $\mathrm{H} 1$ antibody and a matched control antibody together with a FITC-labelled $F\left(a b^{\prime}\right)_{2}$ fragment, as well as by SLE sera testing positive for the LE cell phenomenon and healthy control sera.

Results Apoptosis and necrosis were reliably induced in both PMNC and lymphocytes. The monoclonal anti-histone $\mathrm{H} 1$ antibody bound necrotic cells, but did not bind apoptotic or fresh cells. Likewise, LE cell-positive (and thus anti-histone H1-positive) SLE sera bound to necrotic cells, but not to apoptotic or fresh cells, while healthy sera bound to neither cell.

Conclusion Since, on cells undergoing apoptosis, histone $\mathrm{H} 1$ is not accessible to specific antibodies, necrotic cells or isolated nuclei must be engulfed in the LE cell phenomenon.
P133

Stat1 and phosphorylated Stat1 are increased in lymphocytes and monocytes of patients with systemic lupus erythematosus

M Aringer, T Karonitsch, CW Steiner, E Feierl, G Steiner, JS Smolen

Department of Rheumatology, Internal Medicine III, Medical University of Vienna, Austria.

Arthritis Res Ther 2005, 7(Suppl 1):P133 (DOI 10.1186/ar1654)

Background Both interferon alpha (IFN- $\alpha$ ) and interferon gamma (IFN- $\gamma$ ) are thought to be involved in systemic lupus erythematosus (SLE) immunopathogenesis. In their signal transduction, both cytokines lead to the tyrosine-phosphorylation and consequent nuclear translocation of the transcription factor Signal transducer and activator of transcription 1 (Stat1).

Objective To evaluate Stat1 protein and Stat1 phosphorylation in SLE patients ex vivo and after stimulation with IFN- $\alpha$ as well as IFN- $\gamma$.

Methods Peripheral blood mononuclear cells of 25 patients fulfilling ACR criteria for SLE and of 12 healthy individuals were prepared over Ficoll Paque gradients. Cells were either stained directly after preparation or after $15 \mathrm{~min}$ of incubation in medium with or without the addition of $100 \mathrm{U} / \mathrm{ml}$ IFN- $\alpha$ (Strathmann Biotech) or IFN- $\gamma$ (R\&D Systems). Intracellular staining was performed using either a monoclonal anti-Stat1 antibody and a FITC-labelled rabbit anti mouse antibody (Dako) with the Fix+Perm kit (An der Grub) or a directly PE-labelled monoclonal antiphospho-Stat1 (pStat1) antibody (BD Biosciences Pharmingen) after fixation with $2 \%$ paraformaldehyde and permeabilization with $90 \%$ methanol. After staining, the cells were analyzed on a Becton Dickinson FACScan fluorocytometer. Gates were set for monocytes and for lymphocytes, and the logarithmic mean fluorescence intensity (mfi) was determined.

Results The amount of Stat1 protein, as measured by the mfi, was increased in lymphocytes of SLE patients as compared with healthy lymphocytes $(21.4 \pm 14.9$ [mean \pm standard deviation] versus $7.04 \pm 1.54, P<0.0001, t$ test), and in monocytes from SLE patients as compared with healthy monocytes $(25.1 \pm 13.2$ versus $10.4 \pm 2.54, P<0.0001)$. Lymphocytic and monocytic Stat $1 \mathrm{mfi}$ correlated both for SLE patients (Pearson $r=0.57, P<0.005)$ and for healthy individuals $(r=$ $0.75, P<0.005)$. The amount of phosphorylated Stat1, as measured by pStat1 $\mathrm{mfi}$, was increased in SLE as compared with healthy lymphocytes $(1.63 \pm 0.40$ versus $1.36 \pm 0.22, P<0.02$ ), but not significantly increased in SLE monocytes as compared with healthy monocytes $(4.03 \pm 1.64$ versus $3.24 \pm 1.21, P=$ not significant). Nevertheless, the pStat1 mean fluorescence intensities of lymphocytes and monocytes were highly correlated, both for SLE patients and healthy individuals $(r=0.84, P<0.0001$, and $r=0.91, P<0.0001$, respectively).

As compared with incubation in medium alone, incubation with either IFN- $\alpha$ or IFN- $\gamma$ increased the amount of pStat 1 in SLE lymphocytes (from $1.57 \pm 0.29$ in medium alone to $1.82 \pm 0.36[P<0.01$, paired $t$ test] with IFN- $\alpha$ and to $1.85 \pm$ $0.40[P<0.002]$ with IFN- $\gamma$ ). In contrast, IFN- $\alpha$, but not IFN- $\gamma$, increased the pStat $1 \mathrm{mfi}$ in healthy lymphocytes (from $1.41 \pm 0.15$ in medium alone to $1.83 \pm$ $0.48[P<0.002]$ with IFN- $\alpha$, but to $1.40 \pm 0.18[P=$ not significant] with IFN- $\gamma$ ). Likewise, SLE monocytes increased their pStat1 contents upon incubation with either IFN- $\alpha$ or IFN- $\gamma$ (from $3.74 \pm 1.18$ to $4.66 \pm 1.50[P<0.05]$ and $7.28 \pm$ $4.14[P<0.001]$ for IFN- $\alpha$ and IFN- $\gamma$, respectively), while healthy monocytes responded to IFN- $\alpha$ (pStat1 mfi from $3.52 \pm 1.11$ to $5.23 \pm 2.25, P<0.01$ ), but not IFN- $\gamma$ (pStat1 mfi $3.96 \pm 5.36, P<0.001$ versus medium).

Conclusions Peripheral lymphocytes and monocytes of patients with SLE contain more Stat1 protein than those from healthy individuals and show increased Stat 1 phosphorylation. While healthy peripheral bloob mononuclear cells phosphorylate Stat1 when stimulated by IFN- $\alpha$ only, SLE lymphocytes and monocytes are primed in a way that enables them to also react to IFN- $\gamma$.

\section{P134}

\section{Kinetic and influence of angiogenesis in the course of collagen-induced arthritis}

\section{G Clavel, C Valvason, D Lemeiter, M-C Boissier, N Bessis}

UPRES EA-3408 and Rheumatology Department, University Paris 13 and CHU Avicenne (APHP), Paris, France

Arthritis Res Ther 2005, 7(Suppl 1):P134 (DOI 10.1186/ar1655)

Background Angiogenesis is involved in rheumatoid arthritis since it allows leukocytes and inflammatory mediators infiltration in the synovium. In turn, inflammation also keeps angiogenesis going as inflammatory mediators stimulate endothelial cell proliferation.

Objectives We aimed to evaluate the reciprocical relationship between synovium inflammation and angiogenesis in mice with collagen-induced arthritis (CIA), a model for rheumatoid arthritis.

Methods: CIA was induced by immunization of DBA/1 mice with collagen II in adjuvant. Endothelial cells were detected using a GSL-1 lectin-specific immunohistochemical staining on knee slides. Angiogenesis, clinical scores and histological signs of arthritis were evaluated each week from the induction of $\mathrm{CIA}$ to the 
end of the experiment. Angiogenesis was quantified by counting both the isolated endothelial cells and vessels stained on each slide. To evaluate the effect of an angiogenesis increase on CIA, vascular endothelial growth factor (VEGF) gene transfer was performed with adeno-associated virus VEGF (AAV-VEGF) injection in the muscles of mice with CIA (1010 AAV-VEGF particles intramuscularly 3 weeks before CIA induction). The AAV vectors used in this study contained either mVEGF164 (AAV-VEGF) or lacZ (AAV-lacZ) cDNA, whose expression were driven by the cytomegalo virus promoter.

Results As expected, clinical and histological scores of arthritis, evaluated each week from day 0 to day 55 after induction of $\mathrm{ClA}$, were correlated $(P<0.0001$, $r=0.74$, Spearman correlation test). More importantly, angiogenesis increased as a function of the disease course. A correlation was observed between joint vascularization and clinical scores of arthritis $(P<0.0001, r=0.61)$. A correlation was also shown between vascularization and histological scores $(P=0.0006, r=0.51)$. The overexpression of VEGF induced by gene transfer was followed by an aggravation of arthritis as compared with the AAV-lacZ control group $(P<0.0001$, analysis of variance test). Histological and quantification of angiogenesis are in progress in this experiment.

Conclusion Angiogenesis and inflammation evolved in the same way during the course of CIA. Stimulation of angiogenesis in mice with CIA led to a worse clinical inflammation. These results suggest an early involvement of angiogenesis in joint inflammation development, and emphasize the critical role of angiogenesis in chronic inflammatory arthritis.

Acknowledgement GC was supported by Societe Francaise de Rhumatologie.

\section{P135}

Free radicals regulate glucocorticoid-induced apoptosis in T lymphocytes

PHJ Remans, DM Gerlag, MS Sanders, P Reinders-Blankert,

\section{KA Reedquist, PP Tak}

Division of Clinical Immunology and Rheumatology, Academic Medical Center, University of Amsterdam, The Netherlands

Arthritis Res Ther 2005, 7(Suppl 1):P135 (DOI 10.1186/ar1656)

Background Glucocorticoid-induced apoptosis is a phenomenon of considerable biological and clinical significance. In particular, lymphoid cells are susceptible to glucocorticoid-induced apoptosis. In patients with rheumatoid arthritis, one of the critical hallmarks of synovial T cells is that they suffer from severe oxidative stress. During the past decade, reduction-oxidation (redox) reactions that generate reactive oxygen species (ROS) have been identified as important chemical mediators in the regulation of signal transduction processes. In particular, free radicals appear to have a central role in the balance between cell growth, survival and apoptosis.

Objective To investigate the role of free radicals in corticoid-induced apoptosis. Materials and methods T lymphocytes were isolated from peripheral blood (PB) and synovial fluid through a negative isolation procedure (Dynal). The cells were treated with different concentrations of methylprednisolone, in combination with different inhibitors and substrates of oxidases. Apoptosis was assessed by annexine V/PI staining, and oxidation of the dye DCF was used to measure ROS production. Control, active Ras (RasV12) and inhibitory Rap1 constructs (RapGAP) were introduced in PB T lymphocytes by nucleofection techniques.

Results A time-dependent and concentration-dependent apotosis is induced in T lymphocytes after treatment with methylprednisolone. But where concentrations over $200 \mu \mathrm{M}$ methylprednisolone were required to induce apoptosis in T cells isolated from PB from healthy donors and RA patients, apoptosis in T cells isolated from the synovial fluid could be readily detected at concentrations of $5-10 \mu \mathrm{M}$. To test whether the disturbed redox balance plays a central role in sensitising T cells for glucocorticoid-induced apoptosis, PB T cells were first incubated for 12 hours with subapoptotic concentrations of $\mathrm{H}_{2} \mathrm{O}_{2}(5-50 \mu \mathrm{M})$, and for 48 hours with BSO (which results in intracellular gluthathione depletion) and NAC (which increases intracellular gluthathione). Here, both pretreatment of the T cells with $\mathrm{H}_{2} \mathrm{O}_{2}$ and BSO sensitised the T cells for apoptosis, whereas NAC protected T cells from glucocorticoid-induced apoptosis. Moreover, other $\mathrm{T}$ cells that are known to suffer from oxidative stress also showed increased apoptosis following treatment with methylprednisolone: both HIV-infected CD4 lymphocytes that were isolated from patients diagnosed with AIDS $(n=3)$ and T cells that were nucleofected with RasV12 and Rap1GAP also showed apoptosis at 5-10 $\mu \mathrm{M}$ methylprednisolone. Concomittant with the apoptosis, a 1.5 -fold to 2.5 -fold increase in intracellular ROS production was measured in the pre-apoptotic cells. This increase of intracellular ROS seems to be a critical process in the apoptotic process as incubation in oxygen-deprived conditions inhibited the apoptosis. Also, addition of rotenone and catalase inhibited both ROS increase and apoptosis after methylprednisolone treatment, suggestive of a mitochondrial source of the free radicals. Conclusions Cells suffering from oxidative stress are sensitised to glucocorticoid-induced apoptosis.

\section{P136}

T-cell receptor-independent induction of interferon gamma expression in human memory Th cells

\section{A Sattler}

German Rheumatism Research Center, Germany

Arthritis Res Ther 2005, 7(Suppl 1):P136 (DOI 10.1186/ar1657)

Background In murine Th1 cells, interferon gamma (IFN- $\gamma$ ) expression can be induced by two alternative pathways: a T-cell receptor (TCR)-dependent pathway and an IL-12//L-18-dependent pathway. IL-12/L-18-induced IFN- $\gamma$ production might perpetuate inflammatory loops in autoimmunity leading to chronicity of inflammation. To evaluate a possible role for IL-12/IL-18 induced IFN- $\gamma$ secretion in human autoimmune diseases we analyzed here the requirements for TCR-independent IFN- $\gamma$ expression in human resting memory Th cells mimicking the inflammatory milieu found at the site of inflammation in rheumatoid arthiritis (RA).

Methods Highly purified $\mathrm{CD} 45 \mathrm{RO}^{+}$memory/effector Th cells from healthy blood donors were cultured in the absence of antigen-presenting cells, but in the presence of different cytokine/chemokine combinations known to be overexpressed in the joints of RA patients. After different timepoints cells were analyzed on the single-cell level for the expression of IFN- $\gamma$ and other cytokines. In addition, surface molecules such as activation markers and cytokine receptors were analyzed by FACS. To evaluate intracellular activation pathways, p38 MAP-kinase inhibitors or cyclosporinA were added in some experiments.

Results IFN- $\gamma$ production could be induced, starting after 18 hours, peaking at 36 hours, in a subset of $2-10 \%$ of human memory/effector Th cells with a cocktail of inflammatory cytokines including IL-1 //L-6/IL-7/L-8/L-12/IL-15/L$18 / \mathrm{MIP} 1 \alpha$ and tumour necrosis factor alpha. In contrast to mice, IL-12, IL-18 and an IL-2-receptor common $\gamma$-chain signalling cytokine (IL-2 or IL-7 or IL-15) were determined to be the minimum effective combination. Cytokine-stimulated IFN- $\gamma^{+}$ Th cells did not co-produce IL-2/L-4/L-5/L-10 or tumour necrosis factor alpha. TCR-dependent activation in cytokine-stimulated IFN- $\gamma^{+}$Th cells was excluded as cyclosporinA did not block IFN- $\gamma$ production. However, cytokine-induced IFN- $\gamma$ production was dependent on the p38 MAP-kinase pathway. In contrast to TCRtriggered IFN- $\gamma^{+}$Th cells, cytokine-stimulated IFN- $\gamma^{+}$Th cells did not upregulate 4-1BB (CD137).

Conclusions In this study, we have characterized human cytokine-induced IFN- $\gamma^{+}$ Th cells. Our results should help to clarify the role of inflammatory cytokine networks for the perpetuation of human Th-cell-driven autoimmune disorders such as RA.

\section{P137}

Active immunization (vaccination) against peptides of IL-1 $\beta$ induces self anti-IL-1 $\beta$ antibodies and protects against collagen-induced arthritis

N Bessis' 1 , S Bertin-Maghit² , C Capini², J Chomilier ${ }^{3}$, S Muller ${ }^{4}$, J-F Zagury' ${ }^{2}$, M-C Boissier ${ }^{1}$

1 Université Paris 13 UPRES EA3408 Bobigny and Avicenne AP-HP, Bobigny, France; ${ }^{2}$ Université Paris 6/INSERM EMIO355, Paris, France; ${ }^{3}$ Universités Paris 6 and Paris 4, Paris, France; ${ }^{4}$ UPR 9021 CNRS, IBMC, Strasbourg, France

Arthritis Res Ther 2005, 7(Suppl 1):P137 (DOI 10.1186/ar1658)

Objectives IL-1 $\beta$ is a critical cytokine in inflammation and is involved in the pathogenesis of rheumatoid arthritis. Blocking IL-1 $\beta$ is efficient in the treatment of inflammation. Our goal was to inhibit inflammatory arthritis with a new approach consisting of targeting specific IL-1 $\beta$ peptides in the receptor binding site, and to prove this concept in an experimental model of arthritis.

Methods Epitopes of IL-1 $\beta$ to be blocked were defined in silico by molecular modelling. Synthetized peptides (Fmoc strategy) were linked to KLH. Anti-IL-1 $\beta$ antibodies generated by vaccination of mice against each peptide coupled to $\mathrm{KLH}$ were tested by ELISA and neutralisation assay, determining their ability to inhibit IL-2 production by EL4/NOB1 cells. Collagen-induced arthritis (CIA) was induced in DBA/1 mice with type II collagen in complete Freund adjuvant. Mice were evaluated blindly for clinical arthritis, and joint histology was performed at sacrifice (60 days post-immunization with type II collagen). Mice were immunized by peptide conjugated to KLH 11 weeks prior to induction of CIA. Control groups received $\mathrm{KLH}$ alone or saline.

Results Six peptides were obtained and were able to generate anti-IL-1 $\beta$ antibodies in mice (positive ELISA and neutralization assay). Three of them, chosen on the basis of the ability to generate the highest antibody response, were studied in CIA. One of them (IL-1/36) yielded a significantly improved resolution of $\mathrm{CIA}(P=0.0003$ as compared with $\mathrm{KLH}$ control, analysis of variance); the maximal arthritic score mean was also reduced in the treated group $(9.4 \pm 1.9$ and 14.2 $\pm 1.7, P<0.05)$. Histological analysis showed a clear-cut significant reduction in inflammation $(P<0.001)$ and destruction $(P<0.01)$ scores in the groups treated with IL-1 $1 \beta 6$.

Conclusion Active immunization with targeted peptides of IL-1 $\beta$ was able to generate neutralizing anti-IL-1 $\beta$ auto-antibodies. Vaccination of mice with one of them, IL-1 $1 \beta 6$, was efficient in CIA. 


\section{P138}

Autoreactive $\mathrm{T}$ cells to histone $\mathrm{H} 1$ and core histones in patients with systemic lupus erythematosus GH Stummvoll, R Fritsch, B Meyer, M Aringer, JS Smolen, G Steiner Department of Rheumatology, Internal Medicine III, Medical University of Vienna, Austria

Arthritis Res Ther 2005, 7(Suppl 1):P138 (DOI 10.1186/ar1659)

Background Autoantibodies directed against nucleosomal antigens such as dsDNA and histones are a hallmark of systemic lupus erythematosus (SLE). Histone $\mathrm{H} 1$ constitutes the major antigen in LE cell formation and antibodies to $\mathrm{H} 1$ have been shown to be more specific for SLE than other anti-histone antibodies $[1,2]$. In order to address the role of histone $\mathrm{H} 1$-specific T cells in SLE we investigated the ex vivo cellular reactivity to histone antigen in SLE patients and controls, and characterised $\mathrm{H} 1$-specific T-cell clones.

Methods Peripheral blood mononuclear cells of 39 SLE patients and 20 healthy controls $(\mathrm{HC})$ were exposed to purified histones $(\mathrm{H} 1, \mathrm{H} 2 \mathrm{~A}, \mathrm{H} 2 \mathrm{~B}, \mathrm{H} 3, \mathrm{H} 4)$, and proliferation as well as cytokine production was measured. In addition, $\mathrm{H} 1$-specific Tcell clones were drawn by limiting-dilution cloning of T-cell lines. T-cell phenotyping was done by FACS analysis and the cytokines IL-4, interferon gamma (IFN- $\gamma$ ) and tumour necrosis factor alpha (TNF- $\alpha$ ) were measured in the supernatants by ELISA. Results After stimulation with histone $\mathrm{H} 1$, SLE patients showed a significantly elevated proliferative response as measured by stimulation indices (SI) when compared with $\mathrm{HC}(\mathrm{SI}$ mean $\pm \mathrm{SD}, 2.2 \pm 1.4$ versus $1.5 \pm 0.4, P<0.03)$ and a positive response (i.e. $\mathrm{SI}>2$ ) in 16 patients compared with only two responders among $\mathrm{HC}$ (Fig. 1). The proliferative response to $\mathrm{H} 2 \mathrm{~A}$ was also elevated in SLE patients ( $\mathrm{SI}, 2.9 \pm 2.5$ versus $1.7 \pm 0.8$ in $\mathrm{HC}, P<0.03$ ), whereas the response to $\mathrm{H} 2 \mathrm{~B}, \mathrm{H} 3$ and $\mathrm{H} 4$ did not differ between patients and $\mathrm{HC}$. Cellular responses to $\mathrm{H} 1$ correlated with the presence of anti-histone antibodies but not with clinical features or SLE disease activity. H1-specific T-cell clones obtained from one SLE patient and two $\mathrm{HC}$ showed a Th1-like phenotype producing large amounts of IFN- $\gamma$ and TNF- $\alpha$ but no IL-4.

Conclusion The increased Th1 autoreactivity to histones $\mathrm{H} 1$ and $\mathrm{H} 2 \mathrm{~A}$ in SLE patients suggests that these T cells play an important role in the pathogenesis of SLE not only by driving autoantibody responses but also by virtue of production of the proinflammatory cytokines IFN- $\gamma$ and TNF- $\alpha$.

References

1. Schett G, Steiner G, Smolen JS: Arthritis Rheum 1998, 41:1446-1455.

2. Schett G, Smolen J, Hiessberger H, Hoefler E, Fournel S, Muller S, Rubin RL, Steiner G: Lupus 2002, 11:704-715.

Figure 1

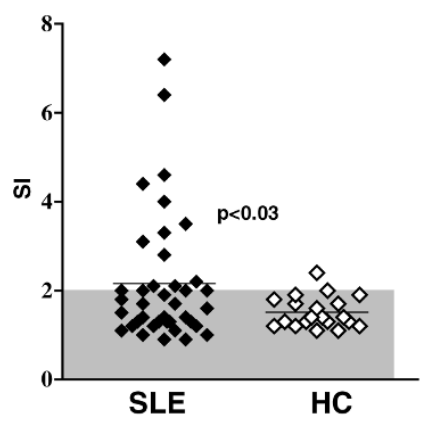

Elevated proliferative responses to histone $\mathrm{H} 1$ in patients with systemic lupus erythematosus (SLE). Proliferation of peripheral blood mononuclear cells from 39 SLE patients and 20 healthy controls $(\mathrm{HC})$ was analysed by measuring $\left[{ }^{3} \mathrm{H}\right] \mathrm{TdR}$ incorporation, and a stimulation index $(\mathrm{SI})>2$ was considered a positive response.

\section{P139}

\section{CCL21 relationship with lymphoid neogenesis and} Iymphatic vascular system in chronically inflamed synovium A Manzo1, S Bugatti², C Buckley³, D Jackson 4 , R Caporali², C Montecucco' ${ }^{2}$ C Pitzalis ${ }^{1}$

${ }^{1}$ Rheumatology Unit, GKT School of Medicine, London, UK; ${ }^{2}$ Cattedra di Reumatologia, IRCCS Policlinico S. Matteo, Pavia, Italy; ${ }^{3}$ Department of Rheumatology, Birmingham University, Birmingham, UK; ${ }^{4}$ Institute for Molecular Medicine, John Radcliffe Hospital, Oxford, UK

Arthritis Res Ther 2005, 7(Suppl 1):P139 (DOI 10.1186/ar1660)

Background CCL21 expression in secondary lymphoid organs is instrumental in stream via PNAd ${ }^{+}$high endothelial venules (HEV). CCL21 is also constitutively produced by lymphatic vessels functioning as a recruiting factor for CCR7 ${ }^{+}$ mature dendritic cells from peripheral tissues to regional lymphoid organs. The same factor participates to the inflammatory cascade being associated with lymphoid neogenetic events and being upregulated in peripheral lymphatic vessels, increasing the magnitude of $\mathrm{T}$-cell response by favouring dendritic cell recruitment to draining lymph nodes.

Objectives In this study we analysed CCL21 protein and mRNA expression in rheumatoid synovium and its relationship with the organizational features of the inflammatory infiltrate and the vascular system (blood and lymphatic vessels).

Methods Thirty-one rheumatoid synovial samples characterized by a variable degree of inflammation and aggregational tendency were analysed by immunohistochemistry and in situ hybridization for CCL21. Molecular features of synovial vessels were analysed by immunohistochemistry for pan-vascular (CD31), HEV (PNAd) and lymphatic (LYVE-1) markers.

Results Two distinct patterns were recognized: vascular and non-vascular. Nonvascular CCL21-producing cells were specifically localized within lymphoid aggregates, frequently surrounding PNAd ${ }^{+}$HEV. This pattern was demonstrated to be similar to human secondary lymphoid organs where, different from the mouse, no CCL21 mRNA was detected directly in HEV. In contrast, CCL21+ vessels did not show a specific association with lymphoid organization, lacking PNAd expression and being recognized inside but also outside perivascular aggregates. Serial section analysis demonstrated the colocalization of CCL21+ vessels with LYVE-1. Conclusions This study emphasizes a differential regulation of CCL21 in the context of synovial lymphoid organization. The anatomical relationship of CCL21producing cells with synovial PNAd ${ }^{+} \mathrm{HEV}$ suggests its involvement in cellular homing although, contrary to the mouse, CCL21 is actually produced perivascularly. Furthermore, CCL21 colocalization with LYVE-1+ lymphatic vessels suggests multiple functions of CCL21 in mediating CCR7 ${ }^{+}$leucocyte synovial homing, intratissue clusterization and promoting exit via lymphatics to draining lymph nodes. These results define CCL21 potential roles in the pathogenetic cascade of rheumatoid synovitis.

\section{P140}

Microparticles from inflammatory cells are strong inducers of matrix metalloproteinases and inflammatory cytokines in synovial fibroblasts

JHW Distler ${ }^{1,2}$, A Jüngel', LC Huber', A Seemayer', RE Gay', BA Michel', JR Kalden ${ }^{2}$, S Gay $^{1}$, DS Pisetsky ${ }^{3}$, O Distler ${ }^{1}$

${ }^{1}$ Center of Experimental Rheumatology, University Hospital Zurich, Switzerland; ${ }^{2}$ Medizinische Klinik III, University Erlangen-Nuremberg, Germany; ${ }^{3}$ Division of Rheumatology, Duke University Medical Center, Durham, North Carolina, USA Arthritis Res Ther 2005, 7(Suppl 1):P140 (DOI 10.1186/ar1661)

Background Microparticles (MPs) are membrane-bound vesicles that are released from cells during cellular activation and apoptosis. Synovial fluids from patients with rheumatoid arthritis contain high numbers of MPs. Here we address the effects of monocyte-derived and T-cell-derived MPs on rheumatoid arthritis synovial fibroblasts (RASF).

Methods Apoptosis of Jurkat T cells, U937 macrophages and primary cells was induced by FasL, actinomycin-D, tumour necrosis factor alpha and staurosporine. Cells were also stimulated with IL-2, concanavalin A, anti-CD3 and lipopolysaccharide. Isolation of MPs was performed by differential centrifugation. The presence of MPs was confirmed by FACS analysis for annexin V and CD3 or CD14. Normal synovial fibroblasts, osteoarthritis synovial fibroblasts and RASF were incubated with MPs for 6-36 hours. Differentially expressed genes were analyzed by real-time PCR and confirmed on the protein level.

Results The release of MPs was induced in apoptotic as well as stimulated cells. Morphological analysis of MPs by electron microscopy showed a population of circular membrane blebs ranging from 200 to $700 \mu \mathrm{m}$. MPs from all cell types induced strongly the synthesis of mRNA for MMP-1 (72 \pm 13-fold), MMP-3 (80 \pm 10 -fold), MMP-9 (18 \pm 4 -fold) and MMP-13 (37 \pm 2 -fold) in RASF in a dose-dependent manner. Similar effects were seen on osteoarthritis synovial fibroblasts and normal synovial fibroblasts. The induction of matrix metalloproteinases (MMPs) was time dependent, with effects primarily seen after 36 hours. The dose-dependent upregulation of biologically active MMPs was also observed on the protein level. The induction was specific for the aforementioned MMPs with no induction seen for MMP-2, MMP-14 or TIMP-1, TIMP-2 and TIMP-3. MPs also increased the synthesis of mRNA for IL-6 (27 \pm 6-fold), IL-8 (35 \pm 3-fold), MCP-1 (5 \pm 1 -fold) and MCP-2 (27 \pm 3 fold) in synovial fibroblasts. No differences in cell viability were observed between fibroblasts stimulated by MPs and controls. In Iא-B-transfected RASF, MMPs were $50-75 \%$ less inducible by MPs compared with wild-type synovial fibroblasts. The stimulation of NF-KB-dependent pathways by MPs was confirmed by EMSA.

Perspective By showing induction of various MMPs and key inflammatory cytokines by MPs, these data provide a novel link between activated and apoptotic inflammatory cells and the invasive potential of synovial fibroblasts in rheumatoid arthritis. 
P141

FcyR-mediated uptake and processing of antigen-immunoglobulin complexes by professional antigen-presenting cells

JMH de Jong', DH Schuurhuis ${ }^{2}$, A loan-Facsinay ${ }^{1,3}$, EIH van der Voort', TJW Huizinga ${ }^{1}$, F Ossendorp ${ }^{2}$, JS Verbeek ${ }^{3}$, REM Toes ${ }^{1}$

${ }^{1}$ Department of Rheumatology, Leiden University Medical Center, Leiden, The Netherlands; ' 2 Department of Immunohematology and Blood Transfusion, Leiden University Medical Center, Leiden, The Netherlands; ${ }^{3}$ Department of Human and Clinical Genetics, Leiden University Medical Center, Leiden, The Netherlands Arthritis Res Ther 2005, 7(Suppl 1):P141 (DOI 10.1186/ar1662)

Rheumatoid arthritis is characterized by the presence of autoantibodies. Various animal models for arthritis have shown a critical role for antibodies in the induction and progression of the disease, with complement and $\mathrm{Fc}_{\mathrm{c}}$ receptors as the key effector mechanisms activated by antigen-antibody immune complexes (IC) in the effector phase of the disease. Although they are required for the perpetuation of the disease, the possible mechanisms by which Fc receptors could contribute to the chronic inflammation in arthritis are still unclear. It is known, however, that crosslinking Fc $\gamma \mathrm{R}$ on effector cells induces a variety of cellular responses, ranging from phagocytosis to secretion of inflammatory mediators and antibody-dependent cell cytotoxicity. Furthermore, FcyRs on dendritic cells (DC) are involved in the enhanced $\mathrm{MHC}$ class II-restricted presentation of antigen from IC to $\mathrm{CD} 4^{+} \mathrm{T}$ cells. Considering that also other antigen-presenting cells (APC) (like B cells and macrophages) express FcyR, this could be a possible mechanism of exacerbating/maintaining inflammation in arthritis. Therefore, we sought to explore the role of $\mathrm{Fc} \gamma \mathrm{R}$ and complement in the $\mathrm{MHC}$ class II-restricted antigen presentation in vivo and to identify the APC involved in this process.

To gain more insight into the ability of various types of APC to take up and present IC to T cells in vivo, we infused ovalbumin (OVA)-IC into naive mice. Direct ex vivo isolation of the different APC subsets showed that only CD11 $\mathrm{c}^{+}$ cells, but not macrophages and B cells, are able to (cross-)present efficiently antigen from IC to $T$ cells, although both macrophages and DC are able to capture IC efficiently. Furthermore, depletion of $\mathrm{CD} 11 \mathrm{c}^{+}$cells, using transgenic mice, abrogated the enhanced presentation of antigen in IC, confirming that DC are the predominant APC involved in (cross-)presentation of IC.

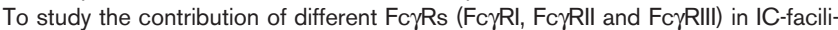
tated antigen presentation, we injected OVA-IC in wild-type mice and FcyR-knockout mice that have received 3 days earlier CFSE-labeled OVA-specific T cells. Our results indicate that activation of OVA-specific T cells in vivo is 10 times more efficient upon uptake of antibody-complexed OVA than soluble OVA. This effect was absent in mice lacking FcyRl, FcyRll and FcyRlll, but not in complement factor 3 knockout mice, indicating that the enhanced efficiency was Fc $\gamma R$ mediated.

Together, these results indicate that the enhanced presentation of antigen in IC and the subsequent activation of $\mathrm{T}$ cells in vivo is mediated primarily by $\mathrm{DC}$, not by macrophages or B cells. Therefore, we consider it more likely that the IC-related contribution of macrophages to rheumatoid arthritis is associated with the release of proinflammatory cytokines and molecules upon activation induced by Fc $\gamma R$ crosslinking, than to the enhanced activation of autoreactive $\mathrm{CD} 4^{+} \mathrm{T}$ cells. The ability to orchestrate and steer the T-cell responses is the responsibility of DC, further emphasizing the crucial role of DC in controlling (ongoing) T-cell responses.

\section{P142}

\section{Treatment of collagen-induced arthritis with cholera toxin-} treated dendritic cells

\section{G Criado, J Inglis, M Feldmann, RO Williams}

Kennedy Institute of Rheumatology Division, Imperial College of London, UK Arthritis Res Ther 2005, 7(Suppl 1):P142 (DOI 10.1186/ar1663)

Dendritic cells (DC) are professional antigen-presenting cells with the unique ability to stimulate naïve $T$ cells and to trigger strong immune responses. However, DC have also shown tolerogenic ability in several systems under certain conditions, depending on their differentiation/activation status. In particular, treatment of DC with cAMP-elevating agents promotes impaired dendritic cell maturation and inhibition of $\mathrm{T}$-cell responses, therefore providing a potential therapy for autoimmune disorders.

Our objective was to study the effect of cholera toxin (CTx)-treated DC in the development of mouse collagen-induced arthritis (CIA), a well-established animal model of rheumatoid arthritis. CTx was chosen because it maintains sustained high cAMP levels by irreversibly binding to adenylate cyclase.

Arthritis was induced by intradermal injection of $200 \mu \mathrm{g}$ bovine type II collagen (bCll) in complete Freund's adjuvant in the base of the tail of male DBA/1 mice. Bone marrow-derived DC, loaded with bCll $(50 \mu \mathrm{g})$ and treated with $\mathrm{CTx}$ $(1 \mu \mathrm{g} / \mathrm{ml})$, were injected at the time of immunization. Arthritis incidence and severity was assessed for 21 days following arthritis onset. For in vitro analysis of the effect of DC in T-cell activation, lymph nodes from bCll-immunized mice were collected before arthritis onset and stimulated with CTx-treated DC. Proliferation and
Figure 1

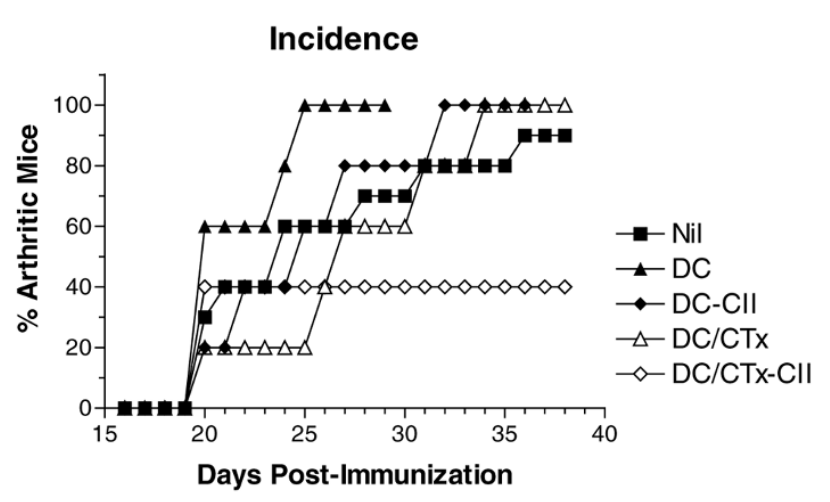

Arthitis incidence in bovine type II collagen (CII)-complete Freund's adjuvant immunized mice injected with cholera toxin (CTx)-treated dendritic cells (DC).

interferon gamma production were determined by thymidine incorporation and capture ELISA, respectively.

Injection of CTx-treated DC resulted in a decreased arthritis incidence (Fig. 1) and reduced severity, compared with non-DC treated animals. This effect required the combination of CTx and antigen specificity, as DC treated with CTx in the absence of bCll, as well as non-treated DC in the presence of bCll, did not show a significant reduction in the incidence of arthritis. Furthermore, treatment with $\mathrm{DC}$ alone results in an acceleration of disease progression.

In vitro analysis of T-cell responses revealed that Ctx-treated DC were able to inhibit both proliferation and interferon gamma production from lymph nodes of immunized mice. Interestingly, this inhibition was not antigen specific, as it was also observed in the absence of bCll, suggesting that additional mechanisms are involved in the reduced arthritis incidence observed in vivo.

In summary, our data indicate that manipulation of dendritic cell activity with cAMP-elevating agents provides a therapeutic tool for the treatment of antigenspecific autoimmune disorders.

P143

Decreased binding of annexin $\mathbf{V}$ to endothelial cells: a novel mechanism of atherothrombosis in patients with systemic lupus erythematosus

A Cederholm 1 , E Svenungsson 2 , K Jensen-Urstad ${ }^{3}, C_{\text {Trollmo }}^{2}$, A-K Ulfgren ${ }^{2}$, J Swedenborg ${ }^{4}, \mathrm{G}^{-Z} \mathrm{Fei}^{2}$, J Frostegård 1

${ }^{1}$ Department of Medicine, CME, Karolinska University Hospital, Huddinge, Sweden; ${ }^{2}$ Department of Medicine, Rheumatology Unit and Center for Molecular Medicine, Karolinska University Hospital, Solna, Sweden; ${ }^{3}$ Department of Clinical Physiology, Södersjukhuset, Sweden; ${ }^{4}$ Department of Surgery, Karolinska University Hospital, Huddinge, Sweden

Arthritis Res Ther 2005, 7(Suppl 1):P143 (DOI 10.1186/ar1664)

Background Patients with systemic lupus erythematosus (SLE) are at high risk of cardiovascular disease, due to atherothrombosis in particular.The mechanisms are not clear; however, recently annexin $\mathrm{V}$, an anticoagulant protein, and antiphospholipid antibodies have been implicated.

Methods Twenty-six women ( $52 \pm 8.2$ years) with SLE and a history of cardiovascular disease (SLE cases) were compared with two age-matched control groups: 26 women with SLE but no cardiovascular disease (SLE controls) and 26 healthy women (population controls). Common carotid intima-media thickness was determined by B-mode ultrasound as a surrogate measure of atherosclerosis. Annexin V binding to human umbilical vein endothelial cells was assessed by flow cytometry after 24-hour culture with plasma. The presence of annexin $\mathrm{V}$ in carotid atherosclerotic plaques was determined by immunohistochemistry. Annexin $V$ levels in circulation and anti-annexin $\lg \mathrm{G}$ and $\lg \mathrm{M}$ antibodies were measured by a commercially available high-sensitivity ELISA. Pooled sera with high capacity to inhibit annexin V binding were preabsorbed against different concentrations of antigens such as cardiolipin and phosphorylcholine and against unrelated antigen tetanus toxoid. Annexin V-binding assay with flow cytometry was performed.

Results Binding of annexin V was significantly lower when plasma from SLE cases was used as compared with controls (SLE cases versus population controls $P=0.002$, SLE cases versus SLE controls $P=0.02)$. There was a striking positive association between annexin $\mathrm{V}$ binding and intima-media thickness $(R=$ $0.73, P<0.001)$ among SLE cases. The annexin $V$ levels were increased in SLE cases compared with both SLE controls and PC $(P=0.03$ and $P=0.004)$, but no differences were detected in anti-annexin V IgG or IgM levels. Depletion of 
IgG from sera with high capacity to inhibit binding of annexin $V$ induced a 2.7-fold increase in binding and preincubation of sera with cardiolipin and phosphorylcholine resulted in increase of median fluorescence intensity of annexin $\mathrm{V}$ binding to human umbilical vein endothelial cells. Immunohistochemical analysis revealed the presence of annexin $V$ in all plaques tested.

Conclusions Decreased annexin $\mathrm{V}$ binding to endothelium caused by immunoglobulin may represent a novel mechanism of atherothrombosis. Increasing annexin $\mathrm{V}$ binding may thus represent a novel therapeutic possibility.

\section{P144}

TLR-9, but not TLR-2, TLR-3 and TLR-4, is upregulated on peripheral blood mononuclear cells of patients with active systemic lupus erythematosus

ED Papadimitraki, E Koutala, G Bertsias, C Choulaki, H Kritikos, P Sidiropoulos, DT Boumpas

Department of Rheumatology, Clinical Immunology and Allergy, Medical School, University of Crete, Greece

Arthritis Res Ther 2005, 7(Suppl 1):P144 (DOI 10.1186/ar1665)

Background Innate immune responses may augment adaptive immune responses via the adjuvant effect of endogenous apoptotic cell death derived nucleic acids that activate Toll-like receptors (TLRs). In animal models of lupus, activation of TLR-9 accelerates renal disease. We studied the expression of TLR2, TLR-3, TLR-4 and TLR-9 in the peripheral blood mononuclear cells from patients with systemic lupus erythematosus (SLE).

Materials and methods Peripheral blood mononuclear cells from 18 SLE patients, four patients with other rheumatic disorders and three normal controls were studied for the expression of TLRs by FACS analysis. TLR-2, TLR-3, TLR-4 and TLR-9 expressions was studied in total lymphocytes, CD19+ and CD14 cells. Disease activity was assessed by the SLEDAl. Patients were divided into those with active/severe disease and those with inactive/mild disease.

Results Eight out of 18 patients had active disease with mean SLEDAI score $12.3( \pm 9.9)$ while 10 had inactive disease with mean SLEDAl score $2.8( \pm 0.9)$. Lymphocyte expression of TLR-9 (mean value and standard deviation) was higher among patients with active/severe disease in comparison with patients with inactive lupus ( $64 \pm 18 \%, n=8$ versus $19 \pm 14 \%, P<0.003$ ) (Table 1 ).

Table 1

\begin{tabular}{lcccc}
\hline & $\begin{array}{c}\text { Active } \\
\text { disease } \\
(n=8) \\
(\%)\end{array}$ & $\begin{array}{c}\text { Inactive } \\
\text { disease } \\
(n=10) \\
(\%)\end{array}$ & $\begin{array}{c}\text { Other } \\
\text { rheumatic } \\
\text { diseases } \\
(n=4) \\
(\%)\end{array}$ & $\begin{array}{c}\text { Healthy } \\
\text { controls } \\
(n=3) \\
(\%)\end{array}$ \\
\hline TLR-9 in lymphocytes & $64 \pm 18$ & $19 \pm 14$ & $22 \pm 21$ & $28 \pm 3$ \\
TLR-9 in CD19+ cells & $42 \pm 20$ & $25 \pm 24$ & $17 \pm 14$ & $33 \pm 5$ \\
TLR-9 in CD14+ cells & $31 \pm 18$ & $16 \pm 13$ & $6 \pm 5$ & $24 \pm 13$ \\
\hline
\end{tabular}

There were no differences between patients and healthy controls regarding the expression of TLR-2, TLR-3 and TLR-4. The expression of TLR- 9 on various B-cell subpopulations of patients with SLE is currently under investigation.

Conclusions TLR-9, but not TLR-2, TLR-3 and TLR-4, is upregulated in peripheral lymphocytes from SLE patients with active/severe disease compared with patients with inactive/mild disease. There is also a trend for increased expression of TLR-9 at least on CD19+ and CD14 ${ }^{+}$cells. Experiments in progress examine the response of these cells to TLR stimulation.

\section{P145}

Bystander-activated CD4+ memory lymphocytes: a role in the pathology of rheumatoid arthritis?

\section{JT Beech, SH Owen, P Green, P Amjadi, FM Brennan}

Kennedy Institute of Rheumatology Division, Imperial College Faculty of Medicine, London, UK

Arthritis Res Ther 2005, 7(Suppl 1):P145 (DOl 10.1186/ar1666)

Background Previous studies in this laboratory have highlighted the importance of the proinflammatory cytokine tumour necrosis factor alpha (TNF- $\alpha$ ) in the pathogenesis of rheumatoid arthritis (RA) and suggested a role for bystanderactivated lymphocytes in its chronic production in the RA joint. Normal peripheral blood (PB) lymphocytes cultured in the presence of a 'cocktail' of inflammatory cytokines (IL-2/L-6/TNF- $\alpha$ ) generated effector cells $\left(T_{C K}\right)$ capable of inducing both immunoglobulin production by B cells [1] and TNF- $\alpha$ production by monocytes in a contact-dependent manner. This was in contrast to lymphocytes activated antigen dependently, via the T-cell receptor $\left(\mathrm{T}_{\mathrm{TCR}}\right)$, which induced cytes isolated from the RA synovial membrane exhibited contact-dependent cytokine induction properties similar to $\mathrm{T}_{\mathrm{CK}}$, not $\mathrm{T}_{\mathrm{TCR}}$, cells [4].

Objective We have examined the population dynamics and phenotypic changes induced in PB lymphocytes during culture with IL-2/IL-6/TNF- $\alpha$ to determine how such cells resemble those found in the RA joint. As the predominant infiltrating lymphocyte population found to accumulate in the RA synovial membrane is the $\mathrm{CD} 4^{+} \mathrm{T}$ cell we have also explored the possibility of generating monocyte TNF- $\alpha-$ inducing effector cells from purified $\mathrm{CD} 3^{+} \mathrm{CD} 4^{+}$populations.

Results Lymphocytes cultured with IL-2/IL-6/TNF- $\alpha$ over 8 days demonstrated a threefold expansion of the natural killer cell population, while the proportion of $T$ cells remained static. However, both these natural killer and T-lymphocyte populations were independently able to induce contact-dependent TNF- $\alpha$ production in monocytes. Interestingly, effector cells generated by culture of the purified $\mathrm{CD}^{+} \mathrm{CD} 4^{+}$fraction with IL-2/L-6/TNF- $\alpha$ for 8 days were able to induce at least threefold more TNF- $\alpha$ production compared with such effectors generated from the corresponding unfractionated lymphocyte population. We subsequently characterised the different naïve and memory cell populations found within the CD4+ fraction of our bystander-activated lymphocyte cultures (as defined by Lanzavecchia and colleagues [5]). Despite an overall net loss in the percentage and number of total $\mathrm{CD} 4^{+} \mathrm{T}$ cells, both naïve $\left(\mathrm{CD} 45 \mathrm{RA}^{+} \mathrm{CCR} 7^{+}\right)$and memory populations (effector:CD45RO ${ }^{+} \mathrm{CRR}^{-}$; central $\mathrm{CD} 45 \mathrm{RO}^{+} \mathrm{CCR} 7^{+}$) were still present after 8 days of culture. However, a preferential retention of effector memory over central memory lymphocytes has been observed in most donors.

Further phenotypic studies have shown that bystander-activated lymphocytes also express high levels of components of the adhesion molecules VLA-1, VLA-4, VLA-5 and LFA-1 ( $\beta 1, \beta 2$ and $\alpha 4)$, but lower levels of L-selectin (CD62L). Such properties are associated with the ability of effector lymphocytes to migrate from lymph nodes and into tissues.

Conclusions Our results suggest that exposure of PB lymphocytes to a cocktail of proinflammatory cytokines induces the outgrowth of an activated effector memory lymphocyte population with the capacity to migrate to inflammatory sites. Studies currently underway will examine the effector function of different $\mathrm{CD}^{+}$ subsets in terms of their ability to induce monocyte TNF- $\alpha$ production.

This work will more closely define pivotal cell types responsible for cognatedependent cytokine production in diseased joints as well as advancing our understanding of the effects and consequences of prolonged cytokine exposure in chronic inflammation.

References

1. Unutmaz D, Pileri $\mathrm{P}$, Abrignani S: Antigen-independent activation of naive and memory resting $\mathrm{T}$ cells by a cytokine combination. $J$ Exp Med 1994, 180:1159-1164

2. Sebbag M, Parry SL, Brennan FM, Feldmann M: Cytokine stimulation of $\mathbf{T}$ lymphocytes regulates their capacity to induce monocyte production of tumor necrosis factor-alpha, but not interleukin-10: possible relevance to pathophysiology of rheumatoid arthritis. Eur J Immunol 1997, 27:624-632.

3. Parry SL, Sebbag M, Feldmann M, Brennan FM: Contact with T cells modulates monocyte IL-10 production: role of T cell membrane TNF-alpha. J Immunol 1997, 158:3673-3681.

4. Brennan FM, Hayes AL, Ciesielski CJ, Green P, Foxwell BM, Feldmann M: Evidence that rheumatoid arthritis synovial $\mathrm{T}$ cells are similar to cytokine-activated T cells: involvement of phosphatidylinositol 3kinase and nuclear factor kappaB pathways in tumor necrosis factor alpha production in rheumatoid arthritis. Arthritis Rheum 2002, 46:3141.

5. Sallusto F, Lenig D, Forster R, Lipp M, Lanzavecchia A: Two subsets of memory $\mathrm{T}$ lymphocytes with distinct homing potentials and effector functions. Nature 1999, 401:708-712

Acknowledgement This work was funded by the Arthritis Research Campaign, UK.

\section{P146}

\section{In vivo induction of foxp 3 in collagen-induced arthritis} treatment with modified dendritic cells

G Falgarone, 0 Jaen, MC Boissier

Rheumatology APHP, UPRES EA-3408, Paris 13 University, Bobigny, France Arthritis Res Ther 2005, 7(Suppl 1):P146 (DOI 10.1186/ar1667)

We studied the prevention of a mice model of rheumatoid arthritis, the collageninduced arthritis (CIA) model, with DNA-matured dendritic cell injection and then studied the induction of Treg through this innate immunity stimulation. CIA was induced as described in DBA/1 mice with bovine type II collagen intradermal injections. Intermediate DNA-matured dendritic cells were injected at D14 intraperitoneally. The clinical course of arthritis was followed and B-cell and T-cell responses were assayed. The induction of $\mathrm{CD} 4{ }^{+} \mathrm{CD} 25^{+}$was tested by flow cytometry and the induction of Treg markers was quantified by quantitative RTPCR. Treatment of CIA with intermediate DNA-matured dendritic cells could prevent arthritis as well as lipopolysaccharide-matured dendritic cells. Neither Bcell and T-cell responses were not modified nor was a $\mathrm{TH} 2$ response observed. 
The induction of Treg $\left(\mathrm{CD} 4^{+} \mathrm{CD} 25^{+}\right)$cells was observed in blood, and lymph nodes. The induction of foxp 3 could be quantified and increased with DNAmatured dendritic cells in peripheral nodes. In conclusion we could observed a prevention of ClA with the injection of DNA-matured dendritic cells that did not modify the specific response against bovine type II collagen. Because of the absence of T-cell and B-cell response modification as well as TH2 modification, we do believed that the induction of $\mathrm{CD} 4{ }^{+} \mathrm{CD} 25^{+}$cells that expressed foxp 3 are involved in the prevention of ClA we observed.

\section{P147}

Investigation of the relationship between the HLADRB1 genotype and the presence of autoantibody to cycliccitrullinated peptide in patients with rheumatoid arthritis M Brózik, Z Weiszhár, JRP Gergely, K Merétey, G Poór

National Institute of Rheumatology and Physiotherapy, Budapest, Hungary

Arthritis Res Ther 2005, 7(Suppl 1):P147 (DOI 10.1186/ar1668)

Background The aetiology of rheumatoid arthritis (RA) has been suggested to be an interaction between genetic and environmental factors. Genetic susceptibility to this disease in most of the population is associated with MHC II molecules that contain an amino acid motif known as the shared epitope (SE). These MHC molecules may bind arthritogenic peptides for presentation to autoreactive $T$ cells. The nature of the arthritogenic peptide is not known, but recent studies have identified post-translationally modified proteins containing citrulline as targets of anti-cycliccitrullinated peptide (anti-CCP) autoantibodies. It has been shown that in HLA$D R B 1^{*} 0401$ transgenic mice the conversion of arginine to citrulline at the peptide side chain position interacting with the SE significantly increases peptide-MHC affinity and leads to the activation of $\mathrm{CD} 4^{+} \mathrm{T}$ cells in the transgene mice.

Objective The aim of this work was to investigate the relationship between the HLADRB1 genotype and the presence of anti-CCP $\lg G$ antibodies in sera of patients with RA.

Methods HLA-DRB1 genotyping was performed using PCR seqence-specific primers from the DR low resolution kit and DRB $1{ }^{*} 01$, DRB ${ }^{*} 04$ subtyping kits as well.

IgG anti-CCP antibody levels were measured by the ImmunoscanRA ELISA kit. Rheumatoid factor was determined by the nephelometric method (Behring). Samples of 131 patients with RA were investigated.

Results SE is present in $75(57.2 \%)$ and absent in $56(42.7 \%)$ patients. The prevalence of anti-CCP autoantibodies is significantly higher in the group of SEpositive patients $(n=44,76 \%)$ than in the group of non-SE carriers $(n=28)$ $(P=0.03$; chi-squared test). The average autoantibody level measured in antiCCP-positive patients carrying SE is $742.7 \mathrm{U} / \mathrm{ml}$ while in the absence of $\mathrm{SE}$ alleles it is $437.5 \mathrm{U} / \mathrm{ml}$, which does not differ statistically.

Conclusion Association of the SE and citrullinated antigens may be one of the triggers initiating the production of anti-CCP antibodies

Acknowledgement This work was supported by the grant OTKA T037876.

\section{P148}

\section{Endothelial function and activation in women with systemic} lupus erythematosus

E Svenungsson, A Cederholm, K Jensen-Urstad, G-Z Fei, J Frostegard

Department of Medicine, Rheumatology Unit, Karolinska University Hospital,

Solna, Sweden

Arthritis Res Ther 2005, 7(Suppl 1):P148 (DOI 10.1186/ar1669)

Background Cardiovascular disease (CVD) is common in systemic lupus erythematosus (SLE) patients although it is not clear whether an increased risk of CVD is a general feature of SLE, or whether this applies only to a subgroup of patients. Objective To measure early signs of atherosclerosis/vascular dysfunction in women with SLE with or without CVD and in controls, and to investigate whether these correlate to circulating markers of endothelial activation.

Methods Twenty-six women with SLE and a history of CVD (defined as a history of objectively verified angina pectoris, myocardial infarction, cerebral infarction or intermittent claudication; age $52 \pm 8.2$ years) were compared with age-matched SLE women without CVD (SLE controls) and population-based age-matched control women (population controls). Flow-mediated dilatation (FMD) of the Brachial artery after reactive hyperemia and after sublingual nitroglycerine administration was performed on women who were not already on nitro-related medication. Levels of thrombomodulin, tumour necrosis factor (TNF) alpha, sTNF receptors, homocysteine and fibrinogen have been associated with endothelial activation/inflammation and were measured in the circulation by use of the ELISA technique.

Results SLE controls and controls did not differ with respect to FMD after reactive hyperemia or after nitroglycerine administration. Only two SLE cases could be investigated for endothelial function because of nitro-related medication. However, levels of TNF- $\alpha$, sTNFR1, sTNFR2, and homocysteine discriminated between SLE cases, SLE controls and population controls $(P<0.05$ for all), whereas thrombomodulin $(P=0.001)$ and fibrinogen $(P=0.02)$ only discriminated between SLE controls and population controls.
Conclusion Women with SLE who did not have manifest CVD did not differ from age-matched controls with respect to FMD, a measure of endothelial dysfunction. Higher levels of circulating markers of endothelial activation/inflammation were present and discriminated between SLE cases, SLE controls and population controls but these did not correlate to measures of endothelia dysfunction, which may not be a general feature of SLE.

\section{P149}

Citrullinated peptide-specific CD19+ $B$ cells are present in synovial tissue and peripheral blood of patients with rheumatoid arthritis

S Ruzickova', Z Cimburek², T Moravcova', 2 Huzlova', I Vesela', J Niederlova' ${ }^{1}$, O Krystufkova', J Vencovsky'

${ }^{1}$ Department of Molecular Biology and Immunigenetics, Institute of Rheumatology, Prague, Czech Republic; 2Department of Gnotobiology and Immunology, Institute of Microbiology, Czech Academy of Sciences, Prague, Czech Republic

Arthritis Res Ther 2005, 7(Suppl 1):P149 (DOI 10.1186/ar1670)

Background Rheumatoid arthritis (RA) is characterized by chronic inflammation of the synovial joints leading to irreversible joint destruction. To prevent and/or slow down progressive joint destruction, the diagnosis of RA at early stage in the disease is important. The autoantibodies against citrullinated proteins (aCCP) provide such ability, representing a marker for early RA. However, a remarkable variety in the reactivity against different citrulline-containing peptides has been described, which indicates that not only citrulline but also its flanking amino acid sequences play a role in the antigenicity and that autoantibodies recognizing such targets are polyclonal. Furthermore, B cells isolated from synovial fluid of aCCP-positive RA patients were shown to produce aCCP antibodies spontaneously, whereas peripheral blood $B$ cells require a stimulation to produce these antibodies. The predominance of IgG production in synovial fluid and synovial tissue was observed in comparison with serum. These findings altogether provide the evidence for the local antigen-driven maturation of $\mathrm{B}$ cells into aCCP-producing plasma cells within inflamed RA synovium due to the presence of citrullinated synovial proteins.

Objective The aim of the study was to analyze the occurence of the aCCP-specific B cells in peripheral blood (PB) and synovial tissue (ST) of RA patients. Three citrulline-containing peptides derived from human natural proteins were used in order to test the hypothesis of polyclonal production of aCCP autoantibodies.

Methods PB and ST were collected from nine patients with RA. Biotinylated citrulline-containing peptides derived from human filaggrin (P1), aggrecan $(P 3)$ and fibrin (P0428), and flow cytometry were employed to analyze B-cell populations specific for citrullinated peptides. The serum levels of aCCP autoantibodies were evaluated by ELISA kit (Mark2). Statistical analysis was performed by Pearson and paired $t$ tests using GraphPad software (GraphPad, San Diego, CA, USA).

Results The serum titres of aCCP autoantibodies ranged from 0.0 to $1300.0 \mathrm{U} / \mathrm{ml}$ (median 276.6; 95\% confidence interval 34.99-880.4). The CD19+ B cells recognizing individual synthetic citrullinated peptides were detected in PB and ST of RA patients, in most cases showing the reaction with at least two of three tested peptides. The frequencies of these B cells differed for both individual citrullinated peptides and patients, especially in ST, and the observed maximum was $2.4 \%$ for peptide $\mathrm{P} 1$ and $\mathrm{P} 3$ in $\mathrm{PB}$ and $6.4 \%$ for peptide $\mathrm{P} 3$ in ST. In some cases, the frequencies of $\mathrm{CD} 19^{+} \mathrm{B}$ cells specific for citrullinated peptides were increased in ST as compared with PB of the same patient; for example, $2.07 \%$ in ST versus $0.75 \%$ in $\mathrm{PB}$ for peptide $\mathrm{P} 0428$ or $6.35 \%$ in ST versus $0.1 \%$ in PB for peptide P3. However, the frequencies of analyzed citrulline-specific B cells did not correlate to serum aCCP autoantibodies titers. The data might indicate the presence of several B-cell subsets specific for different citrullinated substrates within the inflamed joint and the ongoing autoantigen-driven B-cell activation in the synovium. However, the clonal relation between $B$ cells specific for the used individual citrullinated peptides remains unclear and will be subsequently analyzed.

Ackowledgements The authors thank Prof. WJ van Venrooij (University of Nijmegen, Nijmegen, The Netherlands) and Dr JW Drijfhout (Leiden University Medical Center, Leiden, The Netherlands) for the biotinylated citrullinated peptides, provided as a kind gift. This work was supported by grant NK/7273-3 of the Ministry of Health of Czech Republic.

\section{P150}

Identification of genes associated with the presence of ectopic lymphoid structures in rheumatoid arthritis synovial tissues B Baltus', RE Mebius', M Vondenhoff', TWJ Huizinga'2, PP Tak', CL Verweij', TCTM van der Pouw Kraan'1

${ }^{1}$ Department of Molecular Cell Biology and Immunology, VUMC, Amsterdam, The Netherlands; ${ }^{2}$ Department of Rheumatology, LUMC, Leiden, The Netherlands; ${ }^{3}$ Department of Clinical Immunology and Rheumatology, AMC, Amsterdam, The Netherlands

Arthritis Res Ther 2005, 7(Suppl 1):P150 (DOI 10.1186/ar1671)

Rheumatoid arthritis (RA) is a heterogeneous disease, which is also reflected in the type of cellular infiltrates in the RA synovial tissues. In approximately $25 \%$ of 
the patients, the synovial tissue contains cellular infiltrates that have formed organized structures resembling structures normally observed in lymph nodes, comprising follicular dendritic cells (FDCs), with distinct T-cell and B-cell areas, therefore referred to as ectopic lymphoid structures. In the remainder of the patients the tissues do not contain FDCs and show either a diffuse lymphocytic infiltrate or an aggregated T-cell and B-cell infiltrate [1].

The aim of our study is to identify the genes that are expressed in tissues with these ectopic lymphoid structures by cDNA microarray analysis to gain more insight into this specific disease process. We previously showed that the gene expression signatures of synovial tissues from RA patients showed considerable variability, resulting in the identification of distinct molecular forms of RA [2,3]. To evaluate whether the genes involved in the formation of ectopic lymphoid structures could be detected by microarray analysis of whole synovial tissues, we performed an analysis focused on a selection of genes that are known to be involved in normal lymph node development from murine knockout studies.

Indeed, four out of 13 tissues showed an elevated expression of lymph-nodeassociated genes, indicating that microarray analysis of whole synovial tissue allows the detection of these genes. The expression of the FDC-specific marker CD21L and immune histochemical staining of synovial tissue sections confirmed the presence of ectopic lymphoid structures in these tissues. Detailed histochemical analysis further allowed us to subclassify the tissues without lymphoid structures into tissues containing $\mathrm{T}$-cell and $\mathrm{B}$-cell aggregates and tissues with a diffuse type of infiltrate.

Comparison of large-scale gene expression profiles between tissues with the three different types of cellular infiltrates revealed differential expression of genes involved in several processes such as apoptosis, antigen presentation, angiogenesis, chemotaxis and extracellular matrix formation. The results indicate that tissues with ectopic lymphoid structures comprise a distinct type of gene expression that includes a spectrum of genes encoding for adhesion molecules, chemokines, cytokines and their receptors that is required to maintain their highly organized structure.

\section{References}

1. Takemura S, Braun A, Crowson C, Kurtin PJ, Cofield RH, O'Fallon WM, Goronzy JJ, Weyand CM: J Immunol 2001, 67:1072-1080.

2. van der Pouw Kraan TCTM, Van Gaalen FA, Kasperkovitz PV, Verbeet NL, Smeets TJ, Kraan MC, et al.: Arthritis Rheum 2003, 48:2132-2145.

3. van der Pouw Kraan TCTM, Van Gaalen FA, Huizinga TWJ, Pieterman E, Breedveld FC, Verweij CL: Genes Immun 2003, 4:187-196.

\section{P151}

\section{Immunophenotype and functional characteristics of} rheumatoid arthritis derived myeloid dendritic cells and plasmacytoid dendritic cells

\section{S Jongbloed, JA Gracie, RD Sturrock, IB McInnes}

University of Glasgow, UK

Arthritis Res Ther 2005, 7(Suppl 1):P151 (DOI 10.1186/ar1672)

Background Dendritic cells (DC) comprise a heterogeneous network of professional antigen-presenting cells, directly linking innate and adaptive immunity. While implicated in the pathogenesis of different chronic inflammatory arthritides, the analysis of DC subsets has been hampered by a lack of specific DC markers and reliable quantitation. Previously, we have described the significant reduction of circulating peripheral blood (PB) myeloid $\mathrm{DC}(\mathrm{mDC})$ in rheumatoid arthritis (RA) patients and the significant reduction of circulating PB plasmacytoid DC (pDC) in both RA and psoriatic arthritis patients. Furthermore, we have shown that both of these DC subsets are present in synovial fluid (SF) from RA and psoriatic arthritis patients, although $\mathrm{mDC}$ significantly exceed $\mathrm{pDC}$.

Objectives This present study characterises the immunophenotype and functional characteristics of RA-derived $\mathrm{mDC}$ and $\mathrm{pDC}$ in order to assess their potential pathogenic role in arthritis.

Methods $\mathrm{pDC}$ and $\mathrm{mDC}$ were sequentially purified by magnetic cell sorting (MACS) using anti-neuropilin-conjugated magnetic microbeads (pDC) and biotinconjugated anti-CD1c followed by positive selection using anti-biotin-conjugated magnetic microbeads (mDC), from RA PB $(n=3)$ and SF $(n=3)$ and compared with normal healthy controls ( $\mathrm{mDC}, n=4 ; \mathrm{pDC}, n=5)$. Purified cells were phenotyped using CD1c-FITC (mDC only), BDCA-2-FITC (pDC only), CD123-PE (pDC only), CCR5-PE (pDC only), CD11c-PE (mDC only), CD14-PE (mDC only), CD40-APC, CD62L-FITC, CD80-FITC, CD83-APC, CD86-APC, CCR7-PE pre and post 24-hour stimulation with TLR2 agonist $S$. aureus peptiodoglycan (mDC) or TLR9 agonist CpG oligonucleotide (ODN) 2216 (pDC). Supernatants were harvested from all cultures and cytokine concentrations analysed using Luminex. Results Circulating RA PB-derived $\mathrm{mDC}$ and $\mathrm{pDC}$ display a typical immature DC phenotype compared with $\mathrm{mDC}$ and $\mathrm{pDC}$ from healthy control subjects; however, L-selectin (CD62L) expression was significantly decreased on RA pDC $(P=0.0369)$ but not $\mathrm{mDC}(P=0.1573)$. Conversely, while RA SF-derived pDC displayed an immature phenotype directly comparable with their normal PB-
CD86, but decreased CD62L, suggesting a less immature phenotype. Moreover, RA SF-derived $\mathrm{mDC}$ had a higher CD14 expression, suggesting that these cells may be monocyte-derived mDC. FACScan analysis revealed that both SF-derived DC subsets upregulated maturation markers following Toll-like receptor stimulation. Cytokine analysis revealed that while pDC from both normal PB and RA SF produced large levels of interferon gamma and tumour necrosis factor alpha in response to TLR9 stimulation, only RA SF pDC produced low levels of IL-10 and interferon gamma. Similarly, $\mathrm{mDC}$ from both normal PB and RA SF produced large levels of tumour necrosis factor alpha; however, RA SF mDC produced more IL-10 than their normal PB counterparts.

Conclusions We report the ex vivo immunophenotype and functional characteristics of circulating RA PB-derived and SF-derived $\mathrm{mDC}$ and $\mathrm{pDC}$.

\section{P152}

\section{The importance of cell surface RANKL in rheumatoid arthritis}

O Krystufkova1, J Sinkora², K Jarosova', M Hladikova ${ }^{3}$, J Niederlova1, Z Rehakova4, D Tegzova', S Ruzickova', J Vencovsky'

${ }^{1}$ Institute of Rheumatology, Prague, Czech Republic; ${ }^{2}$ DakoCytomation Brno, Czech Republic; ${ }^{3}$ Institute of Medical Informatics 2nd Medical Faculty, Charles University, Prague, Czech Republic; ${ }^{4}$ Military Medical Academy JE Purkyne, Hradec Kralove, Czech Republic

Arthritis Res Ther 2005, 7(Suppl 1):P152 (DOI 10.1186/ar1673)

Rationale Local bone destruction and osteoporosis in rheumatoid arthritis (RA) is mediated by the interaction of the receptor activator of nuclear factor kappa $B$ (RANK) with its ligand (RANKL) and osteoprotegerin (OPG). Effector cells osteoclasts - are generated upon the contact between RANK-positive (RANK+) pre-osteoclasts with RANKL expressed on the surface of osteoblasts or inflammatory cells [1]; a role of the soluble form of RANKL (sRANKL) in synovial fluid (SF) or serum in the osteoclast maturation has also been discussed. We have previously reported higher local production of OPG and SRANKL in RA active joints in comparison with their serum concentration, lower local and systemic concentrations of OPG in RA patients in comparison with those suffering from osteoarthritis (OA) and no difference in SF-sRANKL levels between RA and OA groups [2].

Method Surface expression of RANKL in T lymphocytes and monocytes was evaluated by flow cytometry using goat biotinylated polyclonal anti-RANKL and anti-RANK antisera visualized by streptavidin-phycoerythrin and FITC-conjugated anti-CD3, anti-CD4 and anti-CD14 monoclonal antibodies. Six paired RA samples of SF and peripheral blood were analysed. The results were compared with data from peripheral blood of 18 RA donors, 14 OA donors and 14 healthy donors, respectively.

Results In SF of RA patients, higher numbers (17\%) of CD3+RANKL+ mononuclear cells (synovial fluid mononuclear cells) were found than in peripheral blood mononuclear cell (PBMC) preparations $(5 \%, P=0.07)$. In contrast, relatively more RANKL ${ }^{+}$CD14 ${ }^{+}$monocytes $(79 \%)$ exist in peripheral blood than in SF $(20 \%)$ in the same individuals. When PBMCs in different groups were analysed, significantly higher numbers of $\mathrm{CD}^{+}(8 \%)$ and $\mathrm{CD}^{+}(8.9 \%)$ PBMCs bearing RANKL on the surface were found in the RA group than in controls $(4.3 \%$ and $4.7 \%, P=0.03$ and 0.02 ). In addition, RA patients had significantly higher proportions of CD14+RANKL ${ }^{+}$PBMC (45.6\%) than controls $(30.2 \%, P=0.013)$ or OA patients $(29.7 \%, P=0.046)$. On the other hand, RANK expression on CD14+ PBMC appeared diminished in RA patients (17.3\%) when compared with controls (35.5\%, $P=0.013)$.

Conclusions Our findings suggest that the induction of bone erosions may depend rather on surface-bound RANKL than on its soluble form. This hypothesis is supported by our previous findings (see rationale) as well as by differences in RANKL expression on SF and peripheral blood $\mathrm{CD}^{+}$cells and the increased numbers of RANKL+ lymphocytes in the peripheral blood $\mathrm{CD}^{+}$and $\mathrm{CD}^{+}{ }^{+} \mathrm{com}-$ partments in RA patients. The lower ratio of CD14 ${ }^{+} \mathrm{RANK}^{+}$monocytes in peripheral blood of RA patients could reflect selective recruitment of RANK ${ }^{+}$cells into the sites with elevated RANKL expression. In such a process, a soluble form of RANKL would probably play an important role [3].

1. Kotake S, Udagawa N, Hakoda M, et al.: Activated human T cells directly induce osteoclastogenesis from human monocytes. Arthritis Rheum 2001, 44:1003-1012.

2. Krystufkova O, Niederlova J, Senolt L, et al.: Serum and synovial fluid levels of OPG, but not soluble RANKL, are lower in rheumatoid arthritis than in osteoarthritis patients. In Immunology 2004. Bologna: Medimond International Proceedings; 2004:113-117

3. Breuil V, Schmid-Antomarchi H, Schmid-Alliana A, et al.: The receptor activator of nuclear factor (NF)-kappaB ligand (RANKL) is a new chemotactic factor for human monocytes. FASEB J 2003, 17:1751-1753.

Acknowledgement Supported by grants NK 7293-3 MH CR and NR 7898-3 MH CR. 


\section{P153}

Expression of programmed death (PD)-1 and PD-1 ligands (PD-L1, PD-L2) in peripheral blood mononuclear cells of patients with systemic lupus erythematosus G Bertsias, A Raptopoulou, E Coutala, M Mamoulaki, H Kritikos, P Sidiropoulos, DT Boumpas

Department of Rheumatology, Clinical Immunology and Allergy, Medical School, University of Crete, Greece

Arthritis Res Ther 2005, 7(Suppl 1):P153 (DOI 10.1186/ar1674)

Background Programmed death (PD) -1 is a newly described member of the immunoglobulin super-family that is expressed on activated $T$ lymphocytes and $B$ lymphocytes. Engagement of PD-1 with its specific ligands, PD-L1 (B7-H1) and PDL2 (B7-DC), inhibits lymphocyte proliferation and cytokine expression, and may play a role in peripheral tolerance and negative regulation of T-cell and B-cell responses in vivo. We sought to investigate the expression profiles of PD-1 and PD-1 ligands in peripheral blood cells of patients with systemic lupus erythematosus (SLE).

Materials and methods Blood was drawn from patients with SLE $(n=16)$, rheumatoid arthritis $(n=16)$, other inflammatory disease $(n=4)$, and healthy controls $(n=9)$. Peripheral blood mononuclear cells were separated on a ficolldensity gradient, and flow cytometry analysis was performed using monoclonal antibodies against CD3, CD19, CD14, CD25, CD69, PD-1, PD-L1, and PD-L2. Results See Table 1.

Table 1

\begin{tabular}{|c|c|c|c|c|c|c|c|c|}
\hline & \multicolumn{8}{|c|}{ Disease group } \\
\hline & \multicolumn{2}{|c|}{$\begin{array}{l}\text { Healthy } \\
\text { controls }\end{array}$} & \multicolumn{2}{|c|}{$\begin{array}{l}\text { Systemic } \\
\text { lupus } \\
\text { erythematosus }\end{array}$} & \multicolumn{2}{|c|}{$\begin{array}{l}\text { Rheumatoid } \\
\text { arthritis }\end{array}$} & \multicolumn{2}{|c|}{$\begin{array}{l}\text { Inflammatory } \\
\text { disease }\end{array}$} \\
\hline & Mean & SEM & Mean & SEM & Mean & SEM & Mean & SEM \\
\hline \multicolumn{9}{|l|}{ PD-1+ (\%) } \\
\hline in $\mathrm{CD}^{+}$ & 0.6 & 0.2 & 0.4 & 0.1 & 1.6 & 0.6 & 0.5 & 0.1 \\
\hline in $\mathrm{CD} 19^{+}$ & 1.7 & 0.9 & 1.4 & 0.3 & 0.9 & 0.4 & 1.1 & 0.4 \\
\hline in $\mathrm{CD}^{25^{+}}$ & 3.0 & 1.0 & 2.4 & 0.7 & 3.3 & 1.6 & 8.5 & 3.4 \\
\hline \multicolumn{9}{|l|}{ PD-L1+ (\%) } \\
\hline in $\mathrm{CD}^{+}$ & 2.8 & 1.2 & 4.1 & 0.9 & 2.3 & 0.5 & 2.0 & 0.6 \\
\hline in $\mathrm{CD} 19^{+}$ & 3.9 & 1.3 & 5.1 & 0.9 & 3.7 & 1.0 & 11.3 & 8.0 \\
\hline in $\mathrm{CD} 14^{+}$ & 5.1 & 2.0 & 14.0 & 5.4 & 2.9 & 0.9 & 5.7 & 3.2 \\
\hline \multicolumn{9}{|l|}{ PD-L2+ $(\%)$} \\
\hline in $\mathrm{CD}^{+}$ & 0.6 & 0.2 & 0.5 & 0.1 & 0.5 & 0.2 & 0.4 & 0.0 \\
\hline in $\mathrm{CD} 14^{+}$ & 2.0 & 0.5 & 1.4 & 0.3 & 1.1 & 0.5 & 1.2 & 0.1 \\
\hline
\end{tabular}

SEM, standard error of the mean. No statistically significant differences were observed.

Conclusions In this preliminary report, SLE patients showed a trend for lower expression of PD-1 and higher expression of PD-L1 in unstimulated peripheral blood mononuclear cells compared with other disease controls. These results corroborate findings linking SLE with polymorphism of the PD-1 gene resulting in putative altered expression of the PD-L2 [1]. Lower expression of PD-1 in SLE lymphocytes could be related to ineffective suppression of autoreactive lymphocytes and thus to disease evolution. Currently, we investigate expression of PD-1 and its ligands on subpopulations of lymphocytes $\left(\mathrm{CD} 45 \mathrm{RO}^{+}, \mathrm{CD} 27^{+}\right)$, as well as the kinetics of expression upon stimulation.

Reference

1. Prokunina L, Castillejo-Lopez C, Oberg F, Gunnarsson I, Berg L, Magnusson $\mathrm{V}$, et al:: A regulatory polymorphism in PDCD1 is associated with susceptibility to systemic lupus erythematosus in humans. Nat Genet 2002, 32:666-669.

\section{P154}

Cultured salivary gland epithelial cells from patients with primary Sjögren's syndrome and disease controls are sensitive to signaling via Toll-like receptors 2 and 3: upregulation of intercellular adhesion molecule-1 expression MP Spachidou, EK Kapsogeorgou, EA Bourazopoulou, HM Moutsopoulos, MN Manoussakis

Department of Pathophysiology, Medical School, University of Athens, Greece Arthritis Res Ther 2005, 7(Suppl 1):P154 (DOI 10.1186/ar1675)

Background In previous studies, our laboratory has shown that the salivary gland epithelial cells (SGEC) of patients with primary Sjögren's syndrome (SS) display an intrinsically activated phenotype, as characterised by high expression of adhesion, costimulatory and antigen-presenting molecules. Stimulation of Toll-like receptors (TLRs) expressed by epithelia by native or synthetic ligands of TLR2, TLR3 and TLR4 may be critically involved in the regulation of immune responses through the induction of various immunoactive molecules, such as the intercellular adhesion molecule-1 (ICAM-1/CD54).

Materials and methods Cultured, non-neoplastic SGEC lines derived from minor salivary gland biopsies of patients with SS (SS-SGEC, $n=4)$ and controls $(n=4)$ were studied. The expression of TLR2, TLR3 and TLR4 mRNA was examined by PCR. SGEC were stimulated by $S$. aureus peptidoglycan $(100 \mu \mathrm{g} / \mathrm{ml}$, TLR2ligand), the synthetic analogue of dsRNA poly-inosinic:cytidylic acid (polyl:C) (5 $\mu \mathrm{g} / \mathrm{ml}$, TLR3-ligand) and E. coli lipopolysaccharide (100 ng/ml, TLR4-ligand), for 24 and 48 hours. In certain experiments, cells were also primed with interferon alpha $(500 \mathrm{IU} / \mathrm{ml})$ for 24 hours, prior to the stimulation with polyl:C. The induction of ICAM-1 expression was analysed by flow cytometry on resting and stimulated cells. Results SGEC lines were found to express TLR2, TLR3, and TLR4 mRNA. In agreement with their intrinsic activation status and previously published reports, SS-SGEC lines displayed high constitutive ICAM-1 expression, compared with control-SGEC lines. Triggering of TLR3 with polyl:C had resulted in significantly increased ICAM-1 expression (SS-SGEC: sixfold, controls: sevenfold) that reached maximum expression after 24-hour stimulation. The response to polyl:C was further enhanced after priming of the cells with interferon alpha. Signaling via TLR2 by peptidoglycan was found to upregulate moderately ICAM-1 expression (SS-SGEC: onefold, controls: twofold) after 24-hour stimulation. Despite expression of TLR4 mRNA (by PCR), as well as of surface CD14 protein (by flow cytometry), lipopolysaccharide stimulation failed to upregulate ICAM-1 expression.

Conclusions Our results indicate the SGEC lines are able to respond to synthetic microbial analogues that stimulate TLR2 and TLR3 by upregulating the expression of the adhesion molecule ICAM-1/CD54.

P155

Prevalence of non-rheumatoid arthritis-associated autoantibodies in sera of anti-cyclic citrulline antibodypositive patients

M Brozik, U Böhm, J Csiki, A Polgár, ZS Schmidt, ZS Balogh, M Seszták, J Ortutay, G Bálint, G Hittner, P Bálint, J Korda, L Hodinka, K Meretey

National Institute of Rheumatology, Budapest, Hungary

Arthritis Res Ther 2005, 7(Suppl 1):P155 (DOI 10.1186/ar1676)

Background Serological markers, especially autoantibodies with high disease specificity, have their emphasized role in support of the diagnosis of autoimmune diseases. Detection of anti-cyclic citrulline (anti-CCP) antibodies has gained increasing interest in clinical practice as they have shown to have strikingly high (96-89\%) specificity for rheumatoid arthritis (RA).

Objective The aim of this study is to summarize our experience in testing antiCCP and other autoantibodies on a large number of sera from patients with suspected and definite connective tissue disease.

Methods A total of 2061 sera derived from 1750 patients were requested for detection of anti-CCP and rheumatoid factor (RF) autoantibodies. Conventional autoantibodies were also tested according to the requests of clinicians in cases of 1560 samples. Anti-CCP antibodies were detected with the ImmunoscanRA Elisa kit, and RF by nephelometry (Behring). Antinuclear antibodies were detected by IF test on Hep-2 cells (Inova). Anti-Ena antibodies, anti-DNA, antichromatin, anti-cardiolipin were measured using ELISA kits (Inova). Anti-CCP antibodies exceeding the level of $25 \mathrm{U} / \mathrm{ml}$ were considered positive. Samples expressing antinuclear antibodies at dilution of 1:200 or higher were evaluated.

Results Anti-CCP and RF were present in sera of 331 patients, while 59 patients were positive only for anti-CCP antibodies.

Autoantibody profile detected in anti-CCP-positive and RF-positive patients $(n=$ 331) - antinuclear antibody positive samples, $n=54(16.3 \%)$ : including polimyositis, scleroderma associated antibodies, $n=7(2 \%)$; systemic lupus erythematosus associated antibodies (anti-DNA, anti-chromatin, anti-histone, anti-Sm), $n=12$ (3.6\%); MCTD associated antibodies, $n=5$ (1.5\%); Sjögren syndrome associated autoantibodies, $n=10(3 \%)$; anti-centromere antibodies, $n=4(1.3 \%)$; and primary biliary cirrhosis associated antibodies, antimichondrial antibody, $n=4(1.3 \%)$. In four cases the presence of anti-neutrophyl cytoplasmic antibodies was also seen.

In the group of patients with only anti-CCP positivity $(n=59)-$ two $(3 \%)$ samples were positive for anti-centromer, one of them for Scl70 as well, one was positive for PM/Scl like antibodies, one for anti-DNA, one for anti-Rnp/Sm antibody, and one sample showed strong antimichondrial antibody positivity.

Conclusion These results show a more complex picture of the occurrence of anti-CCP antibodies as they may associate with different stages of connective tissue diseases. We also aimed to initiate further clinical studies to define the role of this family of autoantibodies in the diagnosis of RA-associated secondary diseases, and also in overlap syndromes.

Acknowledgement This work was supported by Hungarian grant OTKA T037876. 


\section{P156}

Identification of hnRNP-A2 (RA33) as a major B-cell and $T$-cell autoantigen in pristane-induced arthritis MH Hoffmann 1,2, J Tuncel ${ }^{3}$, K Skriner 4 , M Tohidast-Akrad ${ }^{5}$, G Schett², JS Smolen ${ }^{1,2}$, R Holmdahl'2, G Steiner ${ }^{1,2}$

${ }^{1}$ Center of Molecular Medicine of the Austrian Academy of Sciences, Vienna, Austria; 2Department of Rheumatology, Medical University of Vienna, Austria; ${ }^{3}$ Section for Medical Inflammation Research, Lund University, Sweden;

${ }^{4}$ Department of Rheumatology, Charité University Hospital, Berlin, Germany;

${ }^{5}$ Ludwig Boltzmann Institute for Rheumatology, Vienna, Austria

Arthritis Res Ther 2005, 7(Suppl 1):P156 (DOI 10.1186/ar1677)

Background Pristane-induced arthritis (PIA) in rats is considered an excellent model for rheumatoid arthritis (RA) since it fulfils the criteria for RA including a chronic relapsing disease course and is not dependent on immunization with exogenous antigen. Although the adjuvant pristane is not immunogenic, the disease is $\mathrm{MHC}$ associated and dependent on the activation of (autoreactive) T cells. However, so far it has not been possible to link the immune response to joint antigens or other endogenous components. HnRNP A2, the RA33 autoantigen (A2/RA33), is a multifunctional RNA binding protein involved in splicing and other aspects of posttranscriptional regulation of gene expression. Autoantibodies as well as autoreactive $T$ cells against A2/RA33 have been found in patients with RA but the pathogenetic role of these autoimmune responses is unresolved [1]. It was therefore the aim of this study to elucidate a potential involvement of A2/RA33 in PIA.

Methods Autoantibodies against A2/RA33 were determined by immunoblotting, and $\mathrm{MHC}$ association of the anti-A2/RA33 immune response was specified by the presence of autoantibodies, delayed-type hypersensitivity reactions and T-cell cytokine secretion in DA rats of different haplotypes. Interferon gamma and tumour necrosis factor secretion by $T$ cells isolated from draining lymph nodes 10 days after pristane injection and restimulation with A2/RA33 in vitro was determined. Expression of A2/RA33 in joints and organs was analysed by immunohistochemistry and western blotting. Nasal vaccinations were performed with A2/RA33 7 days prior to pristane injection.

Results Although anti-A2/RA33 autoantibodies were detected in all four rat strains investigated, the immune response appeared to be particularly linked to the $F$ and $U$ MHC haplotypes. Autoantibodies to A2/RA33 were found in $60 \%$ of DA1.F sera, and $T$ cells of all DA1.F rats tested produced intermediate to high levels of interferon gamma upon stimulation with A2/RA33. The reaction seemed to be entirely produced by $\mathrm{CD} 4^{+}$cells showing a Th1 phenotype. Furthermore, nasal vaccination with A2/RA33 significantly delayed the onset and decreased severity of arthritis in DA1.F rats. Finally, immunohistochemical and western blot analyses revealed pronounced overexpression of A2/RA33 in joints of rats suffering from acute PIA, but not in healthy joints or in joints from animals with chronic PIA.

Conclusion The A2/RA33 autoantigen is targeted by autoantibodies and Th1 cells in rats with PIA shortly after pristane injection. The presence of autoreactive Th1 cells in conjunction with synovial overexpression of A2/RA33 strongly suggests involvement of this autoantigen in the pathogenesis of PIA. This is further bolstered by the observed alleviation and delay of onset of PIA following nasal vaccination with A2/RA33. Thus, A2/RA33 seems to be one of the primary autoantigens in PIA that in conjunction with previous observations on the presence of autoreactive T cells in RA patients may argue for a pathogenetic role also in human RA.

Reference

1. Fritsch $\mathrm{R}$, et al:: Characterization of autoreactive $\mathbf{T}$ cells to the autoantigens heterogeneous nuclear ribonucleoprotein A2 (RA33) and filaggrin in patients with rheumatoid arthritis. $J$ Immuno/ 2002, 169:1068-1076. Acknowledgements This work was supported by a grant from the Austrian Academy of Sciences and by Marie Curie Host Fellowship number HPMT-CT2000-00126 of the European Commission Research Directorate.

\section{P157}

\section{Influence of methotrexate, leflunomide and tumour} necrosis factor alpha inhibitors on immune competence in rheumatoid arthritis patients: a long-run monitoring of Epstein-Barr virus load

\section{N Balandraud ${ }^{1,2}$, S Guis ${ }^{1,2}$, JB Meynard³, I Auger ${ }^{1}$, H Sovran'1, J Roudier ${ }^{1,2}$,} C Roudier ${ }^{1,2}$

${ }^{1}$ INSERM UMR 639, Faculté de Médecine, Marseille, France; ${ }^{2}$ Service de Rhumatologie Est, Hôpital de la Conception, Marseille, France; ${ }^{3}$ Institut de Médecine Tropicale du Service de Santé des Armées, Marseille, France Arthritis Res Ther 2005, 7(Suppl 1):P157 (DOI 10.1186/ar1678)

Background The risk to develop lymphoma is doubled in patients with rheumatoid arthritis (RA) in the the absence of immunosuppressive therapy. This risk is slightly increased by the use of methotrexate or tumour necrosis factor alpha inhibitors (infliximab and etanercept) [1]. Epstein-Barr virus (EBV) is detected in about onethird of lymphoma developing in RA patients. The reason why some patients develop lymphoma is unknown. We have previously shown that RA patients have
(PBMCs), compared with normal controls [2]. RA patients' peripheral blood EBV load is similar to that of healthy transplant recipients (10 copies per $500 \mathrm{ng}$ DNA). In immunosuppressed transplant recipients, elevation of PBMC EBV load above 500 copies/500 ng DNA predicts the emergence of lymphoma.

Objectives We followed RA patients to evaluate the effects of disease-modifying anti-rheumatic drugs (DMARDs) (methotrexate and leflunomide) and tumour necrosis factor alpha inhibitors (infliximab and etanercept) on PBMC EBV load and to predict lymphoma development.

Methods One hundred and nineteen patients fulfilling the 1987 ACR criteria for RA were followed for periods of 6 months -4 years. Twenty patients received methotrexate or leflunomide, eight received only infliximab, 61 received methotrexate and infliximab, and 30 received etanercept. A 214 bp fragment from the conserved long Internal Repeat IR1 was amplified by quantitative PCR to evaluate EBV DNA load. Effect of treatment duration on EBV load was analyzed by the method of generalized estimation equations.

Results EBV load decreased between the beginning and the end of the study in patients receiving DMARDs (mean: 20.24 and 3.27) or etanercept (mean: 4.44 and 0.67). This decrease was significant for DMARDs $(P=0.0038)$. However, EBV load evolution was not significantly related to treatment duration in patients under infliximab (mean: 6.36 and 7.82) or infliximab plus methotrexate (mean: 13.49 and 11.92). Of interest, in one patient receiving infliximab plus methotrexate, EBV load reached 540 copies/500 ng over a short period of time.

Conclusion Methotrexate and etanercept decrease EBV load over time. Infliximab alone or with methotrexate does not influence significantly EBV load. Monitoring EBV load might help detecting the few patients who are likely to develop lymphoma. References

1. Wolfe F, Michaud K: Lymphoma in rheumatoid arthritis. The effect of methotrexate and anti-tumor necrosis factor therapy in 18,572 patients. Arthritis Rheum 2004, 50:1740-1751.

2. Balandraud N, Meynard JB, Auger I, Sovran H, Mugnier B, Reviron D, Roudier J, Roudier C: Epstein-Barr virus load in the peripheral blood of patients with rheumatoid arthritis. Accurate quantification using realtime polymerase chain reaction. Arthritis Rheum 2003, 48:1223-1228. Acknowledgements Supported by PHRC 2003, SFR and ARP.

\section{P158}

\section{A revised assay for the measurement of T-cell receptor excision circles as a quantitative measure of thymic function in systemic lupus erythematosus}

\section{AR Lorenzi, AM Patterson, JD Isaacs}

Musculoskeletal Research Group (Clinical Immunotherapy), Clinical Medical Sciences, University of Newcastle Upon Tyne, UK

Arthritis Res Ther 2005, 7(Suppl 1):P158 (DOI 10.1186/ar1679)

In 1998 Douek and coleagues described a novel approach to measuring thymic output using T-cell receptor excision circles (TRECs) measured in $\mathrm{CD}^{+}$and $\mathrm{CD}^{+} \mathrm{T}$ cells. TRECs are byproducts of T-cell receptor gene re-arrangements, whereby sections of excised DNA re-form as stable episomes that do not replicate with mitosis. TRECs present in T cells arising from the thymus are thus 'diluted out' upon T-cell division and can be used as a surrogate marker of thymic output. However, the interpretation of TREC number in lymphocyte subsets is confounded by the influences of T-cell proliferation, intracellular TREC degradation and T-cell death, and it has been criticised as a measurement of thymic function. Here, we describe a method for quantifying TRECs in whole blood. This revised assay provides a representation of 'total peripheral TRECs'. The effects of T-cell proliferation and cell death on the determined absolute TREC level are minimised, providing us with an improved estimation of thymic function over time. In a series of validation experiments our assay has been shown to have excellent intra-assay and interassay reproducibility and to generate results consistent with previously published work in healthy controls. We are now using this assay to determine thymic function in the autoimmune disease systemic lupus erythematosus to test our hypothesis that a defect in thymic function contributes to lupus pathogenesis.

To measure the absolute TREC number per unit volume of blood (as a representation of 'total peripheral TRECs'), the amount of DNA extracted from a volume of whole blood must be representative of 'total' DNA in that volume of blood. We have compared four methods of DNA extraction from whole blood for consistency of yield and purity. Figure 1a shows the mean DNA yield from parallel extractions $(n=10)$ performed after (a) 1 hour, (b) 6 hours and (c) 24 hours from collection for a single sample. The overall mean yield was $32.23 \mu \mathrm{g} / \mathrm{ml} \pm 2.3$ standard deviations (SDs). Two further, separate, samples were similarly prepared in parallel to confirm consistency - (d) $26.8 \mu \mathrm{g} \pm 1.97 \mathrm{SD}$ and (e) $26.9 \mu \mathrm{g} \pm 2.23 \mathrm{SD}(n=10)$. To measure TRECs in samples we have used a quantitative real-time PCR (TAQMAN ${ }^{\mathrm{TM}}$ ) assay to amplify a known proportion of the extracted DNA. The total TREC number per sample is determined absolutely from a standard curve (derived from a plasmid containing TREC sequence) and final values are presented as TRECs per millilitre of whole blood. Figure 1a (top axis) shows that the TREC number is reproducibly calculated from parallel extractions $(n=5)$. The intra-assay coefficient of variation is $1.4 \%$ (range $0.8-2.5 \%$ ) while the inter-assay co-efficient of variation is $2.4 \%$ (range $0.6-4.4 \%)$. The coefficient of variation is negatively correlated with TREC number $\left(r^{2}\right.$ 
Figure 1

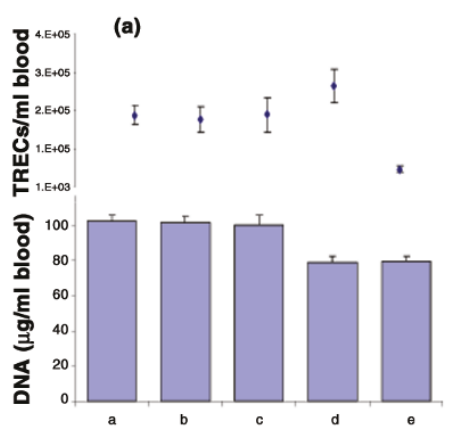

(b)

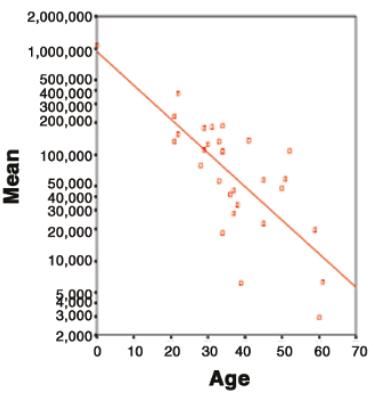

(a) Lower axis: serial DNA extractions (see text). Bars represent mean of $10 \pm$ standard deviation. Upper axis: T-cell receptor excision circles (TRECs) per millitre of blood from the same samples (mean \pm deviation). (b) TRECs per millitre of whole blood declines with age over a range of $\sim 2$ logs from birth.

$=0.83$ and 0.66 , respectively). Using this assay we have also shown that TRECs are reliably detectable in healthy controls (Fig. 1b) and show an age-related decline in keeping with the known reduction in thymic volume over time. TRECs are not detectable in RAJl (human B-cell lymphoma line) or SW48 (human colonic carcinoma cell line) (data not shown). We are now using this assay to determine the TREC number in the autoimmune disease systemic lupus erythematosus to test our hypothesis that a defect in thymic function contributes to lupus pathogenesis. While measurement of TRECs in T cells and their subsets provides a representation of thymic function in the steady state, in the context of autoimmunity, where abundant auto-antigen provides a constant T-cell-activating stimulus, interpretation of the TREC number within lymphocyte subsets is confounded. By reliably and reproducibly estimating total peripheral TRECs we aim to minimise the impact of T-cell turnover on TREC number in order to gain a better understanding of thymic function in systemic lupus erythematosus.

Acknowledgement This research is funded by an arc Clinical Research Fellowship awarded to ARL (Grant number 14490)

\section{P159}

Microarray analysis for molecular characterization of disease activity and measuring outcomes of anti-tumour necrosis factor therapy in rheumatoid arthritis

B Stuhlmüller', T Häupl', N Tandon', M Hernandez'1, C Hultschig², RJ Kuban' ${ }^{1}$, S Salfeld ${ }^{3}$, GR Burmester ${ }^{1}$

${ }^{1}$ Charité, Department of Rheumatology, Berlin, Germany; ${ }^{2} \mathrm{MPI}-\mathrm{MG}$, Berlin, Germany; ${ }^{3}$ Abbott Bioresearch Center, Worcester, Massachusetts, USA

Arthritis Res Ther 2005, 7(Suppl 1):P159 (DOI 10.1186/ar1680)

Introduction In the pathogenesis of rheumatoid arthritis (RA), macrophages (M $\phi)$ play a pivotal role in synovitis, especially at the cartilage-pannus junction. Both $\mathrm{M} \phi$ and peripheral blood monocytes $(\mathrm{MO})$ are activated and release proinflammatory cytokines (IL-1 $\beta$, tumour necrosis factor [TNF] alpha). Neutralization of IL1- $\beta$ and TNF- $\alpha$ leads to clinical improvement in RA.

Objective To characterize MO expression profiles of patients with active RA before and after treatment with adalimumab, a fully human, anti-TNF monoclonal antibody, using microarray analysis. To identify differences in expression of signature genes between patients with uncontrolled, active RA and those in remission induced by anti-TNF therapy.

Methods Genome-wide microarray analyses (HG-U133A/B) were performed to identify signature genes and RA-MO pathways and to establish a customized cDNA array suitable for monitoring anti-TNF therapy. Untouched MO were negatively selected by magnetic cell sorting from $40 \mathrm{ml}$ peripheral blood samples. Total RNA was extracted for CRNA synthesis, and was hybridized with whole genome oligonucleotide and customized cDNA microarrays. The customized cDNA array consisted of $313 \mathrm{cDNAs}$ derived from gene subtraction analysis and from comparative genome-wide U133A analysis. The array includes RA-relevant signature genes, and genes triggered or repressed during anti-TNF treatment. To identify significant gene expressions, bioinformatical MAS 5.0, self-organizing map clustering and predictive analysis for microrrays (PAM) analyses were performed. Results Genome-wide analysis of MO mRNA expression in patients with active RA before and after receiving anti-TNF- $\alpha(n=7)$ revealed significant differences in upregulated and downregulated genes. Self-organizing map analysis revealed six different gene expression clusters. MAS 5.0 and PAM analyses identified 103 differentially expressed genes and permitted the separation of RA patients into two subgroups of 'responders' and 'non-responders', correlating with clinical data (DAS28 and ACR response criteria). Results were confirmed by a customized array using most of the PAM-selected genes. Twenty-four genes were further evaluated by real-time PCR using MO from normal donors and from patients with RA before and during therapy. Signature genes identified were characterized as: disease-relevant genes differentially transcribed in activated RA-MO compared with normal donor MO; genes reversed to 'normal levels' by anti-TNF treatment; or pharmacodynamic marker genes probably indicative of anti-TNF action. Selected genes, which may be indicative for the response to therapy, are currently being further evaluated in extended collections of samples. Conclusions Signatures are important to define MO activation to characterize disease activity and to support therapeutic stratification. The current gene selection could contribute to the investigation of the role of $\mathrm{MO}$ in a wide range of rheumatic diseases and therapeutic intervention, improving rheumatologists' understanding of regulated $\mathrm{MO}$ pathways.

\section{P160}

\section{Amelioration of joint inflammation by a PAR-2-specific monoclonal antibody}

V King1, EB Kelso', JC Lockhart², L Dunning², WR Ferrell', JA Gracie', IB Mclnnes' 1

${ }^{1}$ Centre for Rheumatic Diseases, University of Glasgow, UK; ${ }^{2}$ Biological Sciences, University of Paisley, UK

Arthritis Res Ther 2005, 7(Suppl 1):P160 (DOI 10.1186/ar1681)

Background Protease-activated receptor-2 (PAR-2) is a G-protein-coupled receptor recognized to mediate inflammatory responses. Using a PAR-2 'knockout' mouse, we previously demonstrated this receptor to play a crucial role in chronic joint inflammation [1]. Inhibition of PAR-2 activation therefore potentially represents a novel therapeutic target in treatment of arthritis, but selective antagonists are not yet available.

Objective To test the hypothesis that acute joint inflammation could be inhibited by targeting the 'tethered ligand' sequence of PAR-2 using a specific monoclonal antibody (SAM-11).

Methods The presence of synovial PAR-2 was confirmed in wild-type (PAR-2 $2^{++}$) C57BL/6J mice by western blotting using SAM-11 (Santa Cruz, USA). The antiinflammatory potential of this antibody, which inhibits PAR-2 activation by preventing release of its activating ligand, was investigated by intra-articular administration of SAM-11 to mice prior to induction of acute joint inflammation (under halothane $/ \mathrm{O}_{2} / \mathrm{N}_{2} \mathrm{O}$ anaesthesia) by injection of $20 \mu \mathrm{l} 2 \%$ carrageenan and $4 \%$ kaolin $(\mathrm{C} / \mathrm{K})$ into the knee joint. Joint swelling was assessed by comparing calipermeasured knee joint diameter pre and 24 hours post-injection. The swelling response was compared in untreated, and in two parallel groups of inflamed mice that had received intra-articular injection of SAM- 11 at $5 \mathrm{ng}$ or $10 \mathrm{ng}, 5 \mathrm{~min}$ prior to $\mathrm{C} / \mathrm{K}$ administration. Selectivity of SAM-11 for PAR-2 was confirmed by immunohistochemical analysis (in combination with the Animal Research Kit Dako, USA) of the wild-type mouse brain, which expresses PAR-1, PAR-2, PAR-3 and PAR-4 compared with that of PAR-2 gene-disrupted $\left(\mathrm{PAR}-2^{-l-}\right)$ mice.

Results Western blotting demonstrated the presence of PAR-2 in normal murine synovium and substantial upregulation in acutely inflamed joints. Immunohistochemical analysis of the murine brain with SAM-11 revealed staining in PAR-2+/+ mice but not in PAR-2-/- mice, confirming PAR-2 specificity of this monoclonal antibody. $\mathrm{C} / \mathrm{K}$ resulted in substantial knee joint swelling, which was significantly and dose-dependently $(P<0.00001$; one-way analysis of variance; $n=4$ per group) inhibited by SAM-11 pretreatment (Fig. 1). These findings are consistent with a proinflammatory role for PAR-2 in arthritis, and demonstrate that antibody inhibition of PAR-2 activation ameliorates acute joint inflammation.

Reference

1. Ferrell WR, Lockhart JC, Kelso EB, Dunning L, Plevin R, et al:: Essential role for proteinase-activated receptor-2 in arthritis. J Clin Invest 2003, 111:35-49.

Figure 1

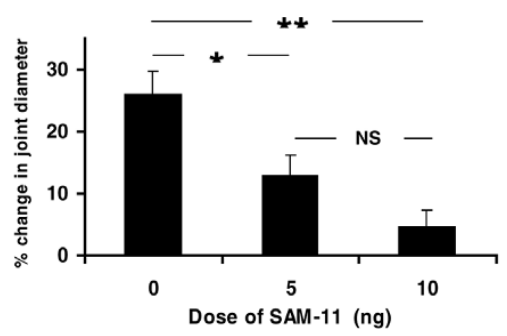

Twenty-four hours after intra-articular injection of kaolin, knee joint swelling is dose-dependently reduced by SAM-11 pre-treatment. ${ }^{\star *} P<0.00001$,

${ }^{\star} P<0.01$ ( $n=4$ per group). NS, no significantly. 
P161

Real-time quantitative PCR used as a biomarker for synovial gene expression after oral prednisolone therapy in patients with rheumatoid arthritis

DM Gerlag', DL Boyle², P Laud 3 , T Nash'3, PP Tak', GS Firestein²

${ }^{1}$ Division of Clinical Immunology and Rheumatology, Academic Medical Center/University of Amsterdam, The Netherlands; ${ }^{2}$ Center for Innovative Therapy, Division of Rheumatology, Allergy and Immunology, UCSD School of Medicine, La Jolla, California, USA; ${ }^{3}$ Astra Zeneca, Macclesfield, UK Arthritis Res Ther 2005, 7(Suppl 1):P161 (DOI 10.1186/ar1682)

The objective of this study was to evaluate the use of real-time quantitative PCR (Q-PCR) using a cellular standard to detect changes in gene expression in synovial tissue samples that could potentially serve as such biomarkers correlating with clinical disease activity. Therefore, the effects of treatment with corticosteroids, a known effective therapy, were analyzed in patients with active rheumatoid arthritis. Patients were randomized to receive either oral prednisolone $(n=10$, $60 \mathrm{mg}$ daily for the first and $40 \mathrm{mg}$ daily for the second week) or placebo $(n=11)$. All patients underwent an arthroscopic synovial biopsy procedure directly before and after 14 days of treatment. Real-time Q-PCR was used to quantify gene expression of tumour necrosis factor alpha, IL-1 $\beta, \mathrm{IL}-6$ and MMP-1 in the synovial tissue samples. The values were expressed as relative units compared with a cellular-based standard. Statistical analysis was performed using an analysis of covariance model. The mean DAS28 was 2.0 units lower (95\% confidence interval, 1.0-3.0) after prednisolone therapy compared with placebo. The mean DAS28 $( \pm$ standard deviation) decreased (from $6.27 \pm 0.95$ to $4.11 \pm 1.43$ ) after prednisolone therapy, but not in the placebo group. For gene expression of IL-8 and MMP-1, the estimated effect of prednisolone compared with placebo was large, and the confidence intervals excluded the likelihood of no effect. A clear trend towards reduction was seen in IL- $\beta$ and tumour necrosis factor alpha mRNA expression in the prednisolone group, but confidence intervals included the value for no effect. The results of this study show that mRNA expression of IL-8 and MMP-1 quantified by Q-PCR may serve as biomarkers in small proof of principle trials designed to screen for potential efficacy in rheumatoid arthritis patients. Acknowledgement The clinical part of study was funded by Astra Zeneca, UK. P162

Expression profiling in synovitis: ranking of candidates, diagnostic performance and individualized interpretation T Häupl', A Grützkau'2, J Grün², V Krenn', M Rudwaleit'1, B Stuhlmüller', C Kaps ${ }^{4}$, J Zacher ${ }^{5}$, G Burmester ${ }^{1}$, A Radbruch ${ }^{2}$

${ }^{1}$ Department of Rheumatology, Charité, Berlin, Germany; ${ }^{2}$ German Arthritis Research Centre, Berlin, Germany; ${ }^{3}$ nstitute of Pathology, Charité, Berlin, Germany; ${ }^{4}$ Oligene GmbH, Berlin, Germany; ${ }^{5}$ Department of Orthopedics, Helios Clinics Berlin Buch, Berlin, Germany

Arthritis Res Ther 2005, 7(Suppl 1):P162 (DOI 10.1186/ar1683)

Objective To provide a systematic overview on expression profiles, to determine a ranking score and to give insight in to the heterogeneity of individual patients within the group of arthritis patients.

Methods Synovial tissue specimens of 10 rheumatoid arthritis (RA), 10 osteoarthritis and 10 normal donors were subjected to GeneChip HG-U133A expression profiling according to the standard protocol, starting with $5 \mu \mathrm{g}$ total RNA and using $15 \mu \mathrm{g}$ cRNA for hybridization. Signals were generated with the GeneChip Operating System and scaled to equal intensities of the whole array. Further analysis included $t$ test and analysis of variance (ANOVA) statistics as well as functional profile component analysis. Classification was performed according to the Prediction Analysis for Microarrays algorithm. Systematic multiple testing with statistics and classification tools was programmed in perl using subgroups of patients and subgroups of genes. Results To characterize the homogeneity of each group, ANOVA and $t$ test statistics were applied using 'leave one out' and 'leave two out' for candidate selection. Subsequently, these one or two donors were tested for the predictive value of the selected candidates. This revealed that one RA donoe, if not participating in the selection process, grouped to osteoarthritis. Analysis for functional profile components showed less infiltration and less inflammation in this donor. However, if this RA donor contributed to the candidate selection, all RA patients were correctly classified. Furthermore, donors of other groups were also classified error-free. This demonstrates that RA with reduced molecular markers of inflammation can still be separated from osteoarthritis and that incorporation of such RA patients in the selection process of candidate genes is mandatory for correct classification.

To characterize the importance of each gene for classification, ranking of candidates was performed according to the significance level by $t$ testing. Multiple subgroups were systematically tested and the ranking of genes was compared. Using an averaged rank list, gene sets were stepwise expanded and systematically tested for classification potential. In addition, the contribution of each gene to the correct classification was assigned to each donor. All together, this information can be visualized on a gene and donor specific way including annotation of significance, classification and proportionate contribution to classification. Conclusion Systematic multiple testing of gene expression profiles provides a precise overview on the quality of array data. It allows ranking of gene candidates, provides insight into patient specific contribution to classification and thus an individualized interpretation of gene expression data.
163

A role for $\mathrm{CD}^{+}{ }^{+}$cells in cell-contact-mediated inflammation SE Robertson', A Crilly', JA Gracie'1, P Life'2, IB McInnes'

${ }^{1}$ Centre for Rheumatic Diseases, Division of Immunology, Infection \& Inflammation, Glasgow, UK; ${ }^{2}$ GlaxoSmithKlein Medical Research Centre, Stevenage, UK Arthritis Res Ther 2005, 7(Suppl 1):P163 (DOI 10.1186/ar1684)

Background While the aetiology of rheumatoid arthritis (RA) is unknown, emerging data suggest that $T$ cell:macrophage cell-cell interactions are critical in disease perpetuation. Cytokine/mitogen-activated $\mathrm{CD} 4^{+} \mathrm{T}$ cells or $\mathrm{CD} 4^{+} \mathrm{T}$ cells isolated directly from RA synovial fluid (SF) are known to induce tumour necrosis factor alpha (TNF- $\alpha$ ) production by monocytes via a cell-contact-mediated mechanism. However, little is known about the ability of $\mathrm{CD}^{+}$cells to drive such effects. Objective To investigate the potential role of $\mathrm{CD}^{+}$cells in cell-contact-mediated stimulation of monocytes, via induction of cytokines. To examine whether $\mathrm{CD}^{+}$ cells isolated from RA SF, like their $\mathrm{CD} 4^{+}$counterparts, have an inherent ability to drive monocyte activation.

Methods $\mathrm{CD}^{+}$or $\mathrm{CD} 4^{+}$cells from healthy volunteer or RA peripheral blood were isolated by immunomagnetic positive selection and activated by phytohaemagglutinin, IL-15 or a cytokine cocktail (IL-6, IL-15 and TNF- $\alpha$ ) for $3 / 6$ days, respectively. SF T cells were purified by immunomagnetic bead separation. T cells were paraformaldehyde-fixed prior to co-culture with autologous immunomagnetically purified CD14-positive cells. CD8 ${ }^{+} / \mathrm{CD}^{+}$cells isolated from RA SF were fixed without prior stimulation. Secreted cytokine production by monocytes was measured by ELISA/Luminex after 48 hours. The purity of isolated $\mathrm{CD} 8^{+} / \mathrm{CD} 4^{+}$populations was assessed by FACS analysis and was routinely $>95 \%$.

Results Mitogen-activated peripheral blood $\mathrm{CD}^{+}$cells induced TNF- $\alpha$ production by monocytes in a cell-contact-dependent manner. Peripheral blood CD8 ${ }^{+}$cells previously activated by IL-15 alone or a cocktail of cytokines similarly stimulated monocyte TNF- $\alpha$ production. Moreover, $\mathrm{CD}^{+}$cells isolated directly from RA SF induced autologous monocyte TNF- $\alpha$ secretion in the absence of prior in vitro activation. Levels of monocyte cytokine production stimulated by $\mathrm{CD} 8^{+}$cells were comparable with levels induced by $\mathrm{CD} 4^{+}$cells in all circumstances investigated.

Conclusion The ability of $\mathrm{CD}^{+}$cells to stimulate monocyte inflammatory cytokine production via a contact-dependent mechanism provides a novel means by which T-cell subsets activated within the local cytokine milieu in RA may contribute to the ongoing disease process. Further understanding of the contribution made by this population to monocyte/macrophage activation is therefore crucial.

P164

Complement and Fc receptor cross-talk in the Arthus reaction: the inflammatory cascade confirmed and refined SR Ali, J Skokowa, N Shushakova, S Konrad, RE Schmidt, JE Gessner

Department of Clinical Immunology, Medical School Hannover, Germany Arthritis Res Ther 2005, 7(Suppl 1):P164 (DOI 10.1186/ar1685)

Complement and $\mathrm{Fc} \gamma$ receptor $(\mathrm{F} c \gamma \mathrm{R})$ effector pathways are central triggers of immune inflammation; however, the exact mechanisms for their cooperation with effector cells and their nature remain elusive. Here we describe a novel regulatory crosstalk between complement and FcyRs on macrophages as the dominant event in the Arthus reaction, the classical animal model of immune complex disease. Specifically, initial contact between immune complexes and macrophages results in cellular regulation: plasma complement-independent C5a production; selective $\mathrm{G}_{\text {-dependent }} \mathrm{C} 5 \mathrm{aR}$ signaling; and $\mathrm{C}_{2} \mathrm{R}-\mathrm{G}_{i}$-mediated $\mathrm{Fc} \gamma \mathrm{R}$ alterations towards $\mathrm{Fc \gamma R}$ RIII, the previously shown main inducer of tumour necrosis factor alpha and CXCR2 ligand production. Distinct inhibitors of this refined inflammatory cascade are each effective in disease prevention, thus indicating cellular components of the C5aR-FcyR axis as potential new therapeutic targets in the treatment of inflammation and autoimmune diseases.

Acknowledgement Supported by the Deutsche Forschungsgemeinschaft DFG 892/8-1 to JEG.

P165

Pharmacokinetic study of oral prednisolone compared with intravenous methylprednisolone in patients with vasculitis of rheumatic disease

KA Rouster-Stevens, JA Daru, LM Pachman, B Javonovic, K-L Ngai

Northwestern University Feinberg School of Medicine \& Children's Memorial

Research Center, Chicago, Illinois, USA

Arthritis Res Ther 2005, 7(Suppl 1):P165 (DOI 10.1186/ar1686)

Introduction Corticosteroids are the drugs of choice for many rheumatic diseases, including vasculitis. There is controversy concerning the route of administration. If there is active vasculitis and the blood vessels of the gastrointestinal tract are affected, there may be impaired absorption of oral corticosteroids.

Purpose To determine whether patients with active vasculitis, as evidenced by elevated neopterin, von Willebrand factor antigen (vWFAg), and abnormal nailfold capillaroscopy (NFC), have equivalent bioavailability of oral prednisolone (OP) compared with intravenous methylprednisolone (IVMP).

Methods Six patients with rheumatic disease involving vasculitis (juvenile dermatomyositis, scleroderma, or overlap syndrome), four females, two males (mean age 17.8 years [range 11-27]; one Hispanic, one Asian, one African American, three Caucasian) were admitted to an IRB-approved Clinical Research Center protocol. After fasting overnight, they received $50 \mathrm{mg} / \mathrm{m}^{2}$ OP on day 1 , and 
$50 \mathrm{mg} / \mathrm{m}^{2}$ IVMP on day 2 . Baseline blood samples were drawn $1 \mathrm{~min}$ prior to each corticosteroid dose (neopterin and VWFAg on day 1; prednisolone level on day 2), at $5,15,30,45,60$, and 90 min and then hourly from the second through the eighth hour. After extraction, samples were analyzed by reverse-phase HPLC for the levels of prednisolone (day 1 samples) and methylprednisolone (day 2 samples). The area under the serum OP or IVMP concentration versus time curve (AUC) was determined using the trapezoid method. NFC images were evaluated by freeze-frame video microscopy as previously described.

Results See Table 1. There was a positive correlation coefficient for $\triangle A \cup C$ (IVMP-OP) with vWFAg, neopterin, and NFC avascularity score of $0.74,0.68$, and 0.66 , respectively; there was a negative correlation coefficient for $\triangle \mathrm{AUC}$ (IVMP-OP) with NFC end row capillary/mm of 0.64 . Linear regression analysis of $\triangle \mathrm{AUC}(\mathrm{IVMP}-\mathrm{OP})$ and $\mathrm{vWFAg}$ approached statistical significance $(P=0.08)$ and given that only six patients were evaluated this may be clinically significant.

Table 1

\begin{tabular}{|c|c|c|c|c|c|c|}
\hline Patient & Diagnosis & 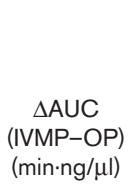 & $\begin{array}{c}\text { Neopterin } \\
\text { (nmol/l) } \\
\text { normal } \\
<10\end{array}$ & $\begin{array}{l}\text { vWFAg } \\
(\mathrm{U} / \mathrm{dl}) / \\
\text { blood } \\
\text { type }^{\mathrm{a}}\end{array}$ & $\begin{array}{c}\text { NFC } \\
\text { end row } \\
\text { capillary/ } \\
\text { mm, } \\
\text { normal } \\
=8-10\end{array}$ & $\begin{array}{l}\text { NFC } \\
\text { avascu- } \\
\text { larity } \\
\text { score, } \\
\text { normal } \\
=0\end{array}$ \\
\hline 1 & JDM & +651897 & 19.5 & $329 / 0$ & 4.08 & 3 \\
\hline 2 & JDM & -21012 & 5.3 & 136/A & 10.49 & 0.5 \\
\hline 3 & JDM & +352690 & 10.7 & $375 / A$ & 4.18 & 2 \\
\hline 4 & JDM & -268415 & 6.9 & 89/B & 14.50 & 1 \\
\hline 5 & Overlap & +924390 & 14.6 & $266 / O$ & 6.28 & 2 \\
\hline 6 & Scleroderma & -70879 & 14.3 & 179/A & 5.16 & 2 \\
\hline
\end{tabular}

AUC, area under the serum oral prednisolone (OP) or intravenous methylprednisolone (IVMP) concentration versus time curve; vWFAg, von Willebrand factor antigen; NFC, nailfold capillaroscopy; JDM, juvenile dermatomyositis. a Normal values per blood type: $A=48-234 ; B=57-241 ; O=36-157$.

Conclusion Patients with elevated vWFAg, neopterin, and/or evidence of abnormal nailfold by capillaroscopy may have decreased absorption of OP. We speculate that this observation can provide the rationale for the greater efficacy of IVMP in the therapy of patients with active vasculitis of rheumatic disease. More patients will be evaluated to further establish the use of IVMP over OP in active vasculitis. Acknowledgements Supported in part by grant M01 RR-00048 from the National Center for Research Resources, NIH, Northwestern GCRC, and the Marlene Apfelbaum Memorial Foundation.

\section{P166}

\section{Characteristics and outcomes in sero-positive late-onset rheumatoid arthritis patients who start a new disease modifying anti-rheumatic drug \\ VK Ranganath}

UCLA, Los Angeles, California, USA

Arthritis Res Ther 2005, 7(Suppl 1):P166 (DOI 10.1186/ar1687)

Background Controversy exists in delineating the characteristics and therapeutics involved in late-onset rheumatoid arthritis (LORA) and younger-onset rheumatoid arthritis (YORA). Many studies have suggested that the disease process of the LORA patient is distinctly different, and thus requires different treatment. Our preliminary work using a rigidly defined, rheumatoid factor (RF)-positive rheumatoid arthritis prospective cohort of 263 patients suggests that LORA and YORA patients are similar after correcting for age-related factors, for example erythrocyte sedimentation rate and C-reactive protein. In the same cohort of patients, $\mathrm{Wu}$ and colleagues recently demonstrated that the presence of both shared epitope-containing DRB $1{ }^{*} 04$ alleles and novel RANKL polymorphisms was associated with an 18-20 year earlier onset of rheumatoid arthritis in YORA patients and did not predict increased severity of disease.

Objective Aim 1 To demonstrate that RF-positive YORA and LORA patients with the same disease duration have similar baseline disease-related characteristics, after adjusting for age-related processes. Aim 2 To compare outcomes of RFpositive LORA and YORA patients with the same disease duration, after accounting for age-related processes.

Hypothesis $2 A$ Responses to disease-modifying anti-rheumatic drug treatments will be similar between LORA and YORA patients with equivalent disease duration. Hypothesis 2B RF-positive LORA and YORA patients with equivalent disease duration will have comparable side effects to disease-modifying anti-rheumatic drugs.

Methods This study will use the Consortium of Rheumatology Researchers of North America and the Rheumatoid Arthritis Disease-Modifying Anti-Rheumatic Drug Intervention and Utilization Study 1\&2 databases, consisting of 15,000 rheumatoid arthritis patients. Patients with known RF positivity will be included in the cohort, with LORA $\geq 60$ and YORA $<60$ years of age. We will control for duration by stratification. We will use two methods to demonstrate similarity between YORA and LORA subjects: equivalence testing and confidence intervals. Alternate statistical analyses include generalized matching by the propensity scores method and regression analyses.

Significance LORA patients have been treated inconsistently and with much apprehension, due to uncertainty about the diagnosis of LORA and concern about prescribing toxic medications to the elderly. With strictly defined cohorts showing similar characteristics/outcomes, treatment of the LORA and YORA patients by physicians should be similar.

\section{P167}

\section{Toll-like receptor 4 polymorphisms and ankylosing spondylitis}

\section{R Adam, JA Gracie, RD Sturrock}

Centre for Rheumatic Diseases, Division of Immunology, Inflammation and Infection, Glasgow Royal Infirmary University Trust, Glasgow, UK Arthritis Res Ther 2005, 7(Suppl 1):P167 (DOI 10.1186/ar1696)

Introduction Ankylosing spondylitis (AS) is a chronic systemic rheumatic disorder, which is characterised by sacroilitis, enthesopathy, and a variety of extraarticular manifestations. Despite having the strongest association ever described with a tissue antigen, HLA B27, the pathogenesis of AS remains poorly understood. Immunoregulatory genes and Gram-negative gut bacteria are thought to be important in disease expression. It is now known that mammalian immune response to Gram-negative bacteria is mediated by Toll-like receptor 4 (TLR4), a pattern recognition receptor. Two cosegregating missense mutations have recently been described in TLR4 that lead to a diminished host response to Gram-negative bacteria. We hypothesise that TLR4 mutations occur with an increased frequency in HLA B27-positive individuals who develop AS than in healthy HLA B27-positive controls, and allow increased survival and the systemic distribution of Gram-negative gut bacteria to the joints. This study aims to compare the frequency of two common TLR4 mutations (Asp299Gly, and Thr399lle) between AS patients and HLA B27 healthy controls.

Methods The TLR4 genotypes of over 100 patients and 100 HLA B27 healthy controls were determined using allele-specific PCR and restriction fragment length polymorphism analysis. The allele frequencies were compared using a chisquared test of association.

Results There was no significant difference between the frequency of the Asp299Gly allele or the Thr399lle allele in AS and healthy HLA B27 controls. Conclusion Two common TLR4 polymorphisms, which cause a functional deficiency in host immune response to Gram-negative bacteria, are not significant in AS.

\section{S9}

Follow up study of B-lymphocyte depletion in the treatment of patients with systemic lupus erythematosus MJ Leandro, JCW Edwards, MR Ehrenstein, G Cambridge, DA Isenberg

Centre for Rheumatology, University College London, London, UK

Arthritis Res Ther 2005, 7(Suppl 1):S9 (DOI 10.1186/ar1712)

Background Systemic lupus erythematosus (SLE) is an autoimmune rheumatic disease in which B-lymphocytes play an pivotal role. We have induced B cell depletion utilising a combination of rituximab, cyclophosphamide, and steroids given intravenously. This regime depletes B-lymphocytes in the peripheral blood. We now report a long-term follow up study of 24 patients who had failed conventional immunosuppression.

Methods 24 patients ( 22 female, 2 male, mean age 30 years, range 17-49) were treated. The mean disease duration was 7.9 years (range 1-18 years). Twelve of the patients were Caucasian, 8 Afro-Caribbean and 4 of Asian origin. The majority of these patients were treated with two infusions of $1 \mathrm{~g}$ rituximab, two infusions of $750 \mathrm{mg}$ cyclophosphamide and two infusions of methylprednisolone $100 \mathrm{mg}$. Patients were allowed to continue on prednisolone and hydroxychloroquine. Only 2 patients continued on azathioprine.

Results The global BILAG score reduced from a median of 13.9 at baseline (SEM = $1.18)$ to $5(S D=1)$ at three months and $5.0(S E M=0.6)$ at six months. $23 / 24$ patients achieved depletion in the peripheral blood (CD19+<0.005/l) and there was improvement in BILAG score in each of the eight systems or organs. The period of $B$ lymphocyte depletion ranged from three to eleven months except in 1 patient who remains depleted for $>4$ years. Whereas the mean total serum immunoglobulins levels remained within the normal range, analysis of the serum C3 levels (in 21 patients) showed a statistically significant improvement $(P<0.0005)$ at six months as did anti-dsDNA binding $(P<0.02) .1$ patient, scored the most active (BILAG global score 45) died of sepsis five months after the infusion having shown a considerable improvement at three months. 1 patien had a severe infusion reaction. No other serious adverse events were observed. 7 patients have now been retreated, 1 on two further occasions. 1 patient failed to deplete on retreatment due to a documented specific HACA response.

Conclusion $\mathrm{B}$ lymphocyte depletion therapy utilising rituximab has been shown to be an effective treatment for active refractory lupus. Our data further indicate that both clinical and serological improvement is seen in the majority of these patients. The efficacy and apparent safety of this treatment in established cases of lupus indicate the possible use of this treatment in patients earlier in the course of their disease. 